\title{
Implementing a Large-scale Electronic Health Record System in the Primary Healthcare Centres in Saudi Arabia
}

\author{
Alzghaibi, Haitham A.
}

How to cite:

Alzghaibi, Haitham A. (2019) Implementing a Large-scale Electronic Health Record System in the Primary Healthcare Centres in Saudi Arabia. Doctoral thesis, Swansea University.

http://cronfa.swan.ac.uk/Record/cronfa52386

Use policy:

This item is brought to you by Swansea University. Any person downloading material is agreeing to abide by the terms of the repository licence: copies of full text items may be used or reproduced in any format or medium, without prior permission for personal research or study, educational or non-commercial purposes only. The copyright for any work remains with the original author unless otherwise specified. The full-text must not be sold in any format or medium without the formal permission of the copyright holder. Permission for multiple reproductions should be obtained from the original author.

Authors are personally responsible for adhering to copyright and publisher restrictions when uploading content to the repository.

Please link to the metadata record in the Swansea University repository, Cronfa (link given in the citation reference above.)

http://www.swansea.ac.uk/library/researchsupport/ris-support/ 


\section{Implementing a Large-scale Electronic Health Record System in the Primary Healthcare Centres in Saudi Arabia}

By

Haitham Ali M. Alzghaibi

Submitted to Swansea University in fulfilment of the requirements for the Degree of Doctor of Philosophy

Swansea University

2019 


\begin{abstract}
There is worldwide demand for the implementation of electronic health systems and a transformation to electronic transactions in healthcare organisations. This move to ehealth transformation stems from the perceived positive impact that e-health systems have in improving the quality of healthcare and, in turn, reducing expenses. Despite this, more than half of previous Electronic Health Record System (EHRS) implementation projects have failed due to several barriers and challenges. There has been no previous research that has explored the implementation of an EHRS in Primary Healthcare Centres (PHCs). In addition, barriers and facilitators to the implementation of large-scale EHRS in PHCs are not well defined and there is little known about the impact of Financial Resources (FR) and Centralised Management (CM) on such implementation. Thus, this thesis aims to explore the large-scale implementation of EHRS in PHCs in Saudi Arabia (SA). To achieve this aim, a mixed-methods approach comprising both quantitative and qualitative methods was adopted. Data were collected via questionnaire-based studies and semistructured interviews. Three different populations were targeted: project team members, PHC staff, and EHRS end-users. Descriptive and inferential statistics were applied to the quantitative data, and thematic analysis was used to analyse the qualitative data. The findings revealed high PHCs readiness at the organisational and individual level when compared with the technological level. Both FR and CM were documented to have a positive impact on the implementation of a large scale EHRS. Several facilitators to the implementation of the EHRS were identified, including: strong leadership and appropriate management, PHC specifications, system usability, perceived usefulness and efficiency. The scale of the project, shortage in Health Informatics (HI) expertise, lack of training and support, geographic challenges, software selection and end-user involvement were identified as the main barriers to implementing a large-scale EHRS in the PHCs. No relationships were detected between individual demographic differences, such as age and gender, and level of readiness or satisfaction. Based on the Saudi experience, there may be some important transferable lesson for similar projects elsewhere. Large-scale EHRS projects need to adopt CM. In addition, due to shortage in HI expertise, policymakers may need to carry out some consultations to formulate good implementation plane. Largescale projects also need to be implemented by more than one vendor and include training and technical support to increase end-user satisfaction. Inadequate infrastructure, lack of interoperability, changing executives and lack of technical support were the main possible causes to the failure of large-scale EHRS projects. Implementation needs to ensure sufficient budget and time have been allocated to mitigate the challenges identified.
\end{abstract}




\section{DECLARATION}

This work has not previously been accepted in substance for any degree and is not being concurrently submitted in candidature for any degree.

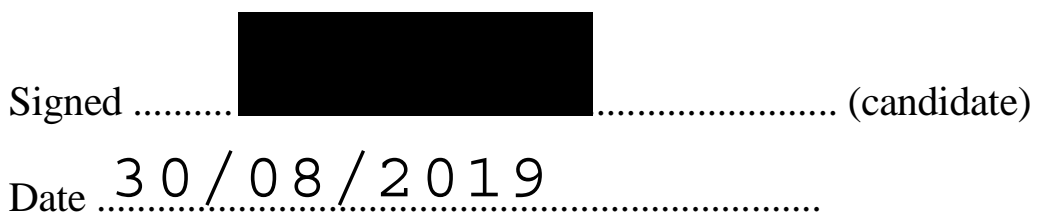

\section{STATEMENT 1}

This thesis is the result of my own investigations, except where otherwise stated. Where correction services have been used, the extent and nature of the correction is clearly marked in a footnote(s).

Other sources are acknowledged by footnotes giving explicit references. A bibliography is appended.

Signed

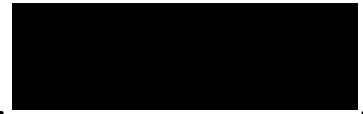
(candidate)

Date $30 / 08 / 2019$

\section{STATEMENT 2}

I hereby give consent for my thesis, if accepted, to be available for photocopying and for inter-library loan, and for the title and summary to be made available to outside organisations.

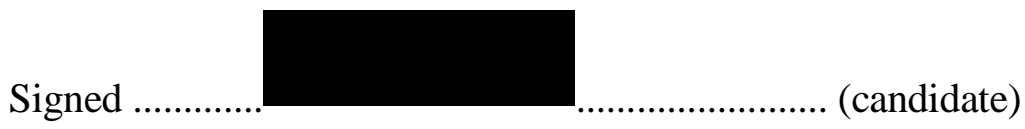

Date $30 / 008 / 2019$

NB: Candidates on whose behalf a bar on access has been approved by the University (see Note 7), should use the following version of Statement 2:

I hereby give consent for my thesis, if accepted, to be available for photocopying and for inter-library loans after expiry of a bar on access approved by the Swansea University.

Signed (candidate)

Date 


\section{Acknowledgements}

I wish to express my sincerest gratitude to my supervisory team Professor Gareth Jenkins, Professor Hayley Hutchings and MR. Anthony Paget for their insightful and invaluable contributions. This $\mathrm{PhD}$ would not have been possible without their support, guidance and encouragement. I would like to give my deep thanks to all of them not only for their excellent academic guidance but also for their emotional support and understanding during the last four years. Next, I would like to extend a special thanks to my parents for their patience of being away of them during my study, and for their sincere prayers and motivation words. I would also like to express my love and gratitude to my wife Nouf Altuaymi and my kids Ali and Mohammed who accompanied me during my $\mathrm{PhD}$ journey, with their love and support, I was able to complete my thesis. I also would like to express my sincerest gratitude to Professor Saleh Aldhamegh who contribute to this achievement. Special and great thanks to him, this achievement could not have been possible without his unbounded and ongoing support in my postgraduate education journey. A super thanks to the inter-observer Dr. Mohammed Alwaheeb, he spent very long time analysing my qualitative data to improve the validity of the result. 


\section{List of publications}

Alzghaibi, H., Jenkins, G., \& Hutchings, H. (2018). FACILITATORS TO LARGESCALE IMPLEMENTATION OF THE ELECTRONIC HEALTH RECORD SYSTEM IN THE PHCS IN SAUDI ARABIA: MIXED-METHODS. Paper presented at the 3rd International Saudi Health Informatics Conference (ISHIC), Saudi Arabia, Riyadh.

Alzghaibi, H., Jenkins, G., \& Hutchings, H. (2018). READINESS OF THE SAUDI PRIMARY HEALTHCARE CENTRES TO LARGE-SCALE IMPLEMENTATION OF THE ELECTRONIC HEALTH RECORD SYSTEM: PRACTITIONERS PRESPECTIVE. Paper presented at the 3rd International Saudi Health Informatics Conference (ISHIC), Saudi Arabia, Riyadh. 
Table of Contents

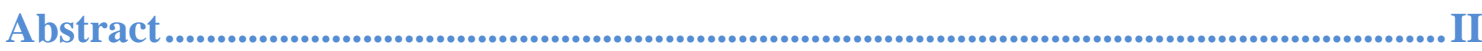

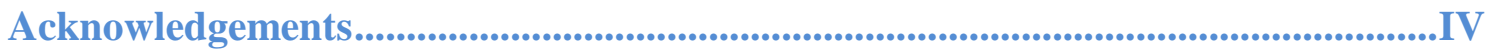

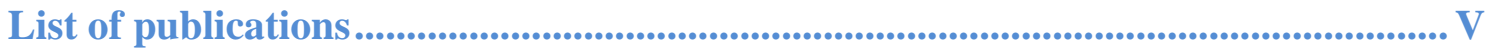

Table of Contents ................................................................................................................... VI

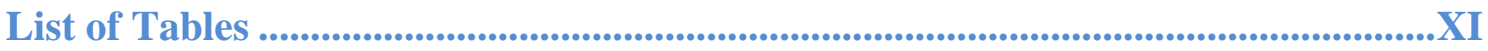

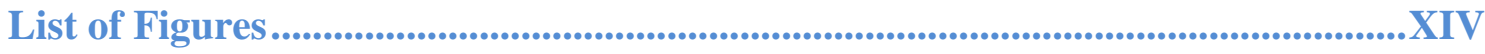

Glossary of Terms ...................................................................................................... XV

Table of Abbreviations .............................................................................................................XVIII

1 Chapter One: Introduction and Background ........................................... 1

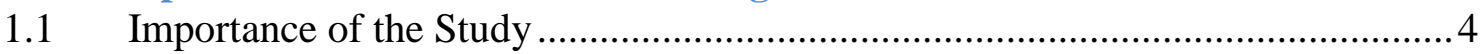

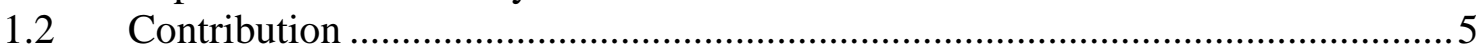

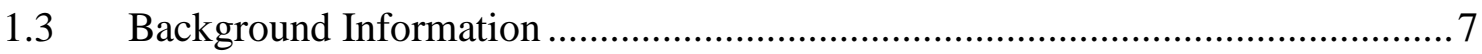

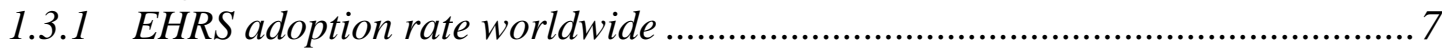

1.3.2 Electronic Health Record System (EHRS) _................................................. 7

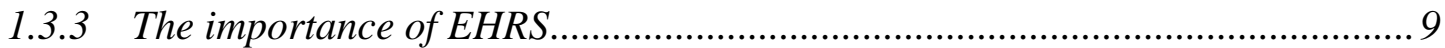

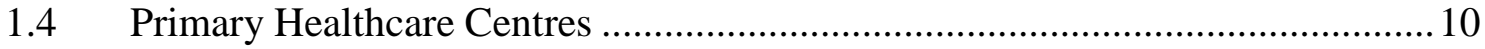

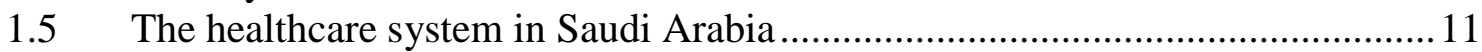

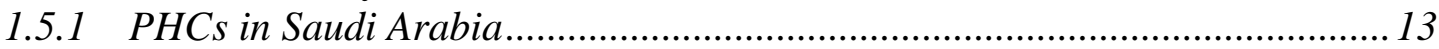

1.5.2 Centralised Management (CM) in SA healthcare .......................................... 14

1.6 ICT development in the SA healthcare system ............................................. 14

2 Chapter Two: Literature Review .................................................................... 17

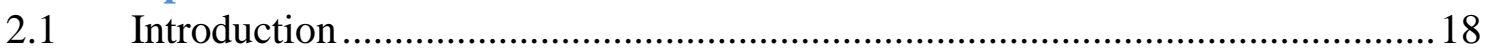

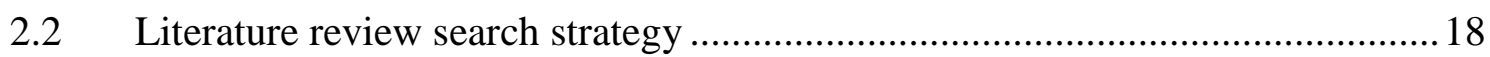

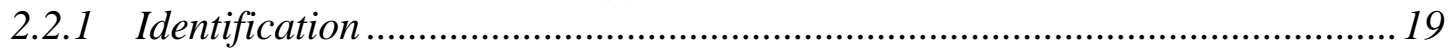

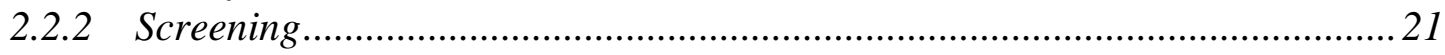

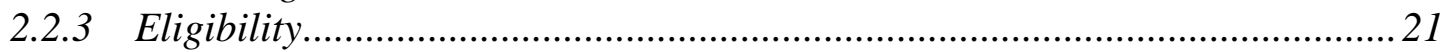

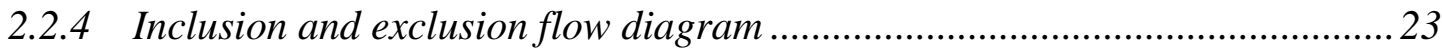

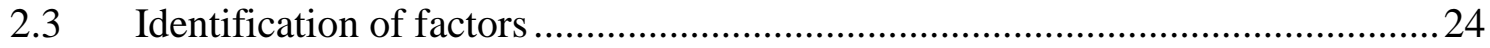

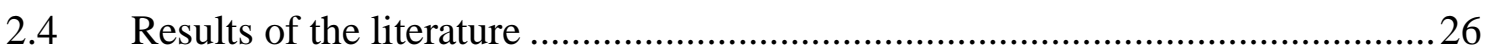

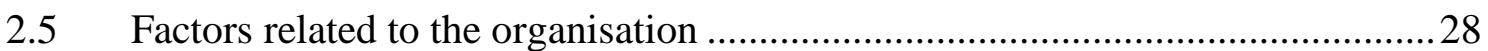

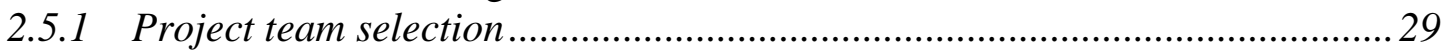

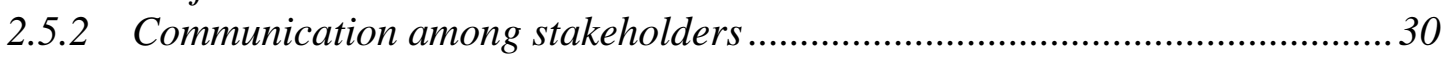

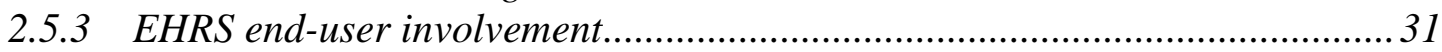

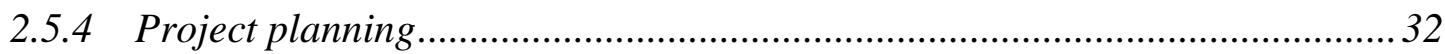

2.5.5 Healthcare organisation readiness and workflow analysis ............................... 34

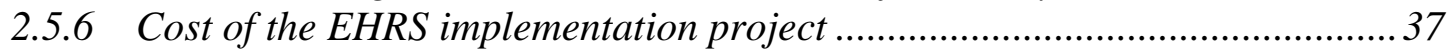

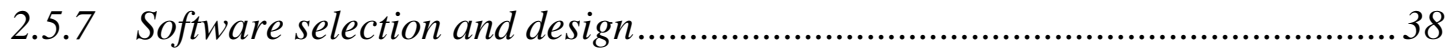

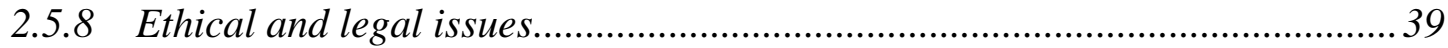

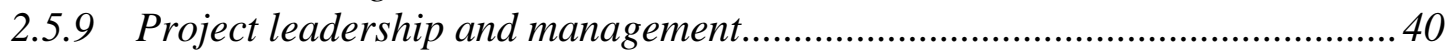

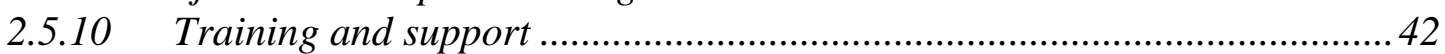

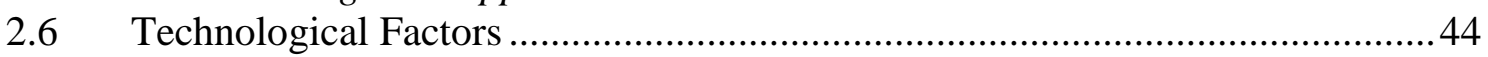

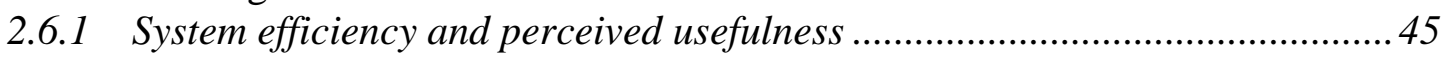

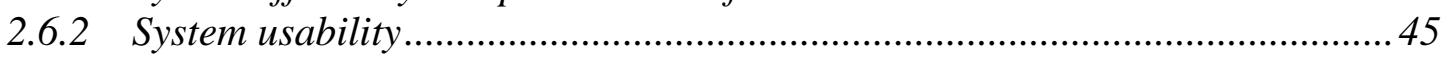

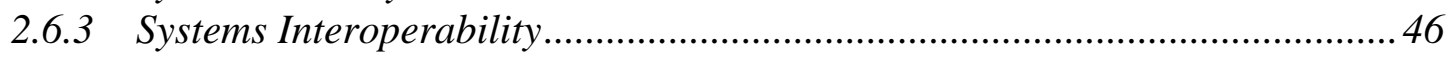




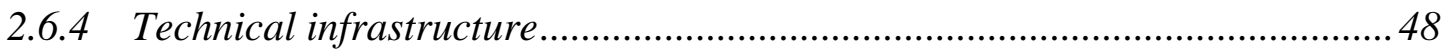

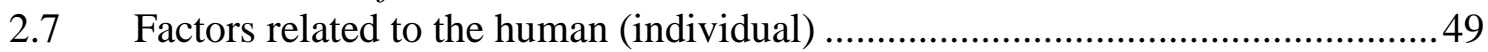

2.7.1 Individual readiness for EHRS implementation............................................49

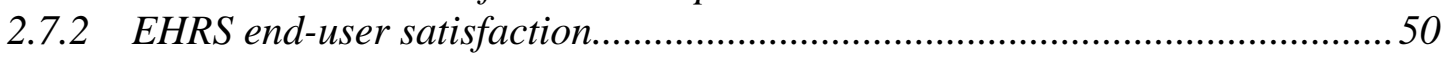

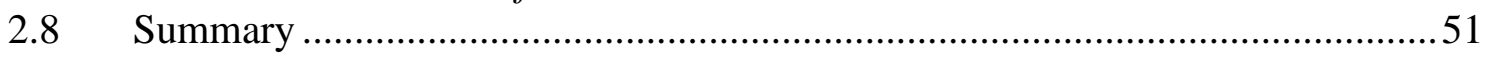

3 Chapter Three: Research Methodology .................................................. 55

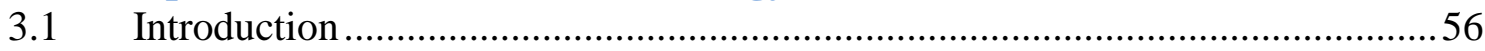

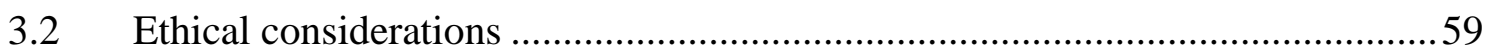

3.3 Study One: Assessing PHCs readiness for the implementation of the EHRS from

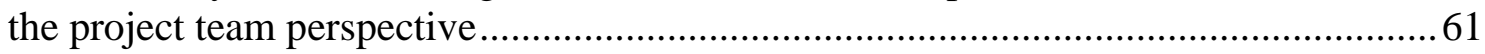

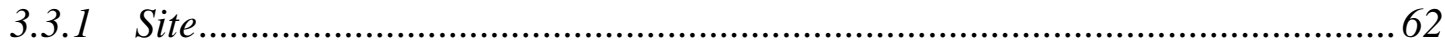

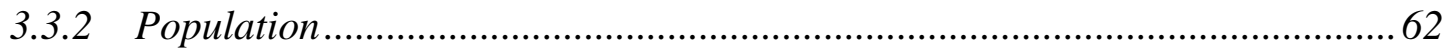

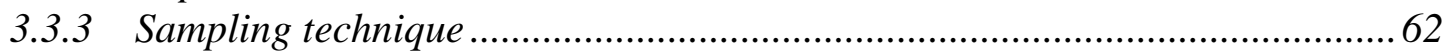

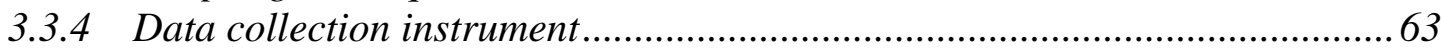

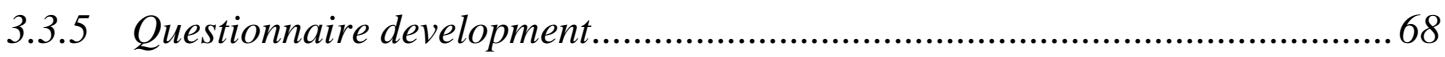

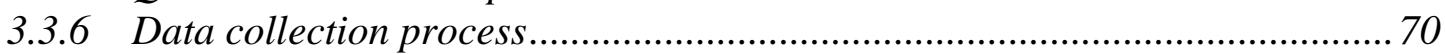

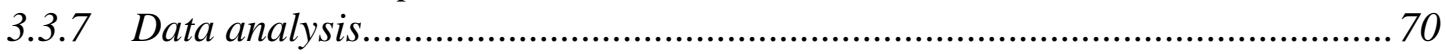

3.4 Study Two: Assessing PHC readiness for EHRS implementation in SA from a

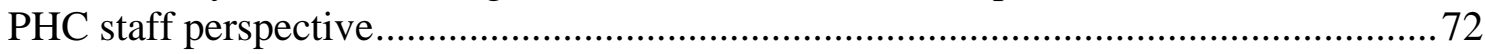

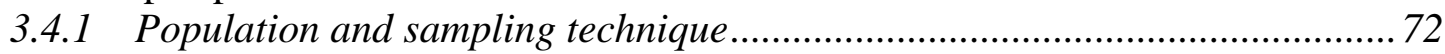

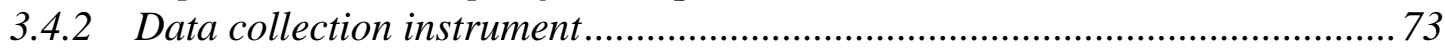

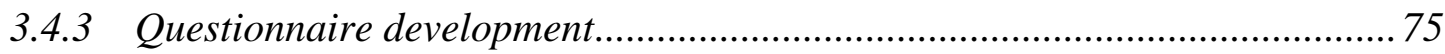

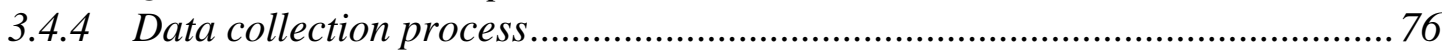

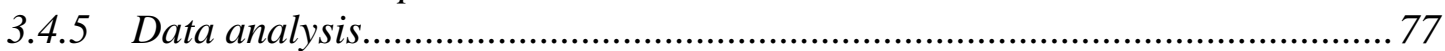

3.5 Study Three: Evaluation of implemented EHRS in PHCs in SA from the

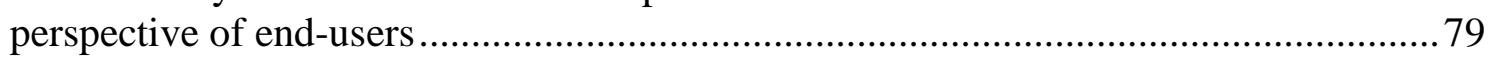

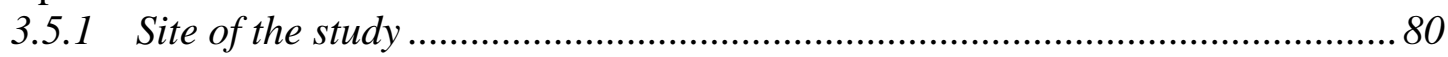

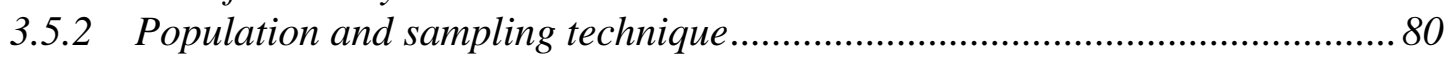

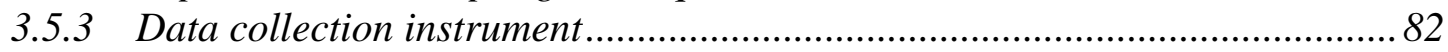

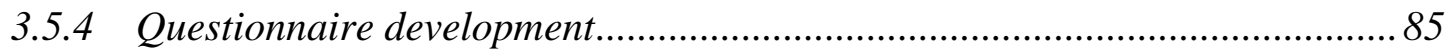

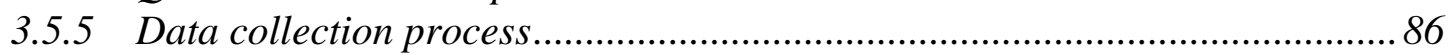

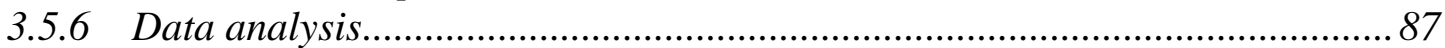

3.6 Study Four: Semi-structured interviews to explore EHRS implementation in

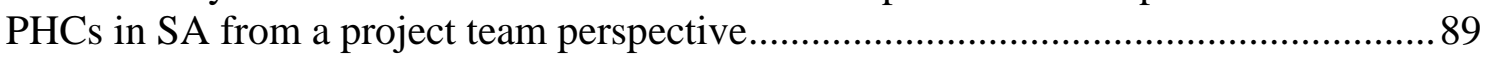

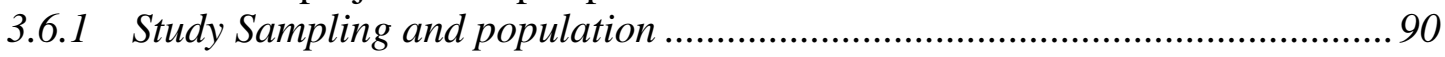

3.6.2 Data collection via semi-structured interviews...............................................90 90

3.6.3 Data collection process and the interview guide ......................................... 91

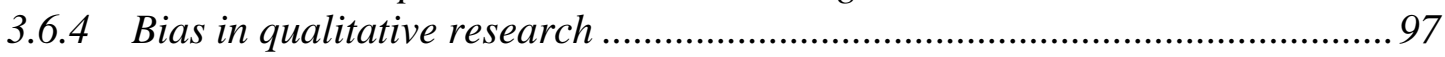

3.6.5 Qualitative data analysis of semi-structured interviews.............................. 99

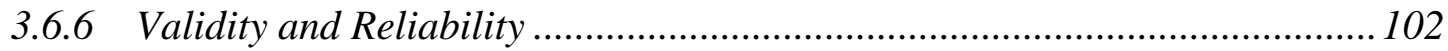

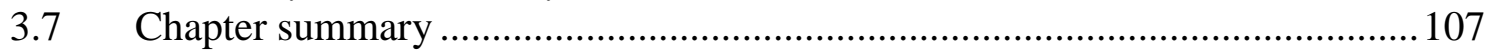

4 Chapter Four: Study One: Assessing PHC Readiness for EHRS

Implementation from the Project Team Perspective........................................108

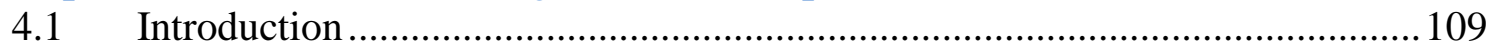

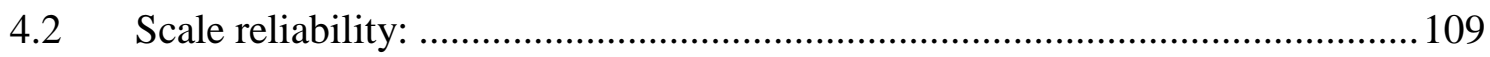

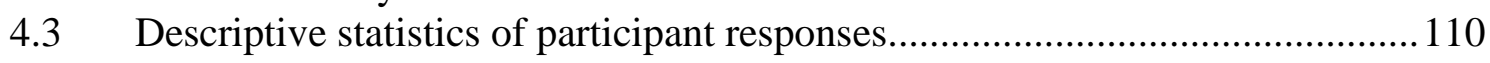

4.3.1 Organisational Information Technology/Systems Innovation Readiness Scale (OITIRS).....

4.3.2 The impact of CM on EHRS implementation PHCs in SA:........................ 127 
4.3.3 The impact of financial resources (FR) on EHRS implementation in PHCs in SA 129

4.4 Summary

5 Chapter Five: Study Two: Assessing PHCs Readiness for EHRS

Implementation in SA from a PHC Staff Perspective ..................................... 132

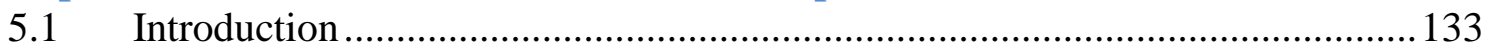

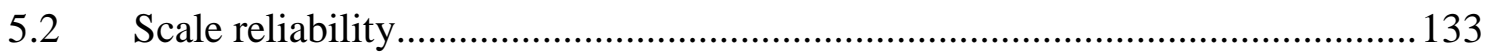

5.3 Descriptive statistics of participant responses................................................. 134

5.3.1 Participant perceptions of the perceived usefulness of an EHRS ................. 136

5.3.2 Participant responses regarding perceived readiness for EHRS

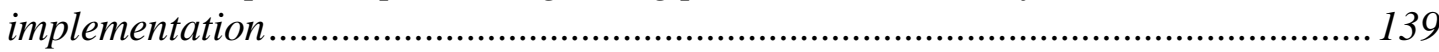

5.3.3 Participant responses regarding endorsement of EHRS implementation .... 141

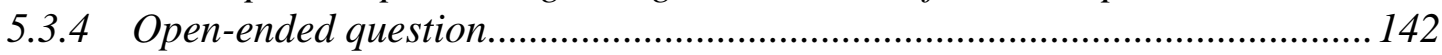

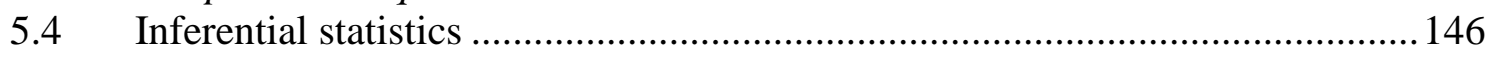

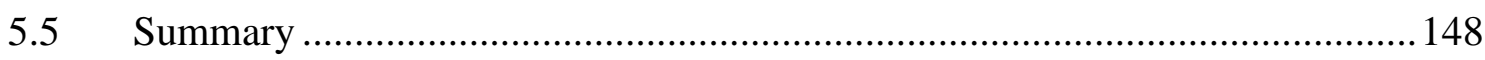

6 Chapter Six: Study Three: Evaluation of the Implemented EHRS in PHCs in

SA from the Perspective of End-users........................................................... 149

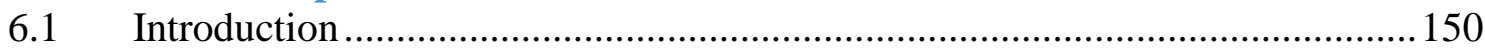

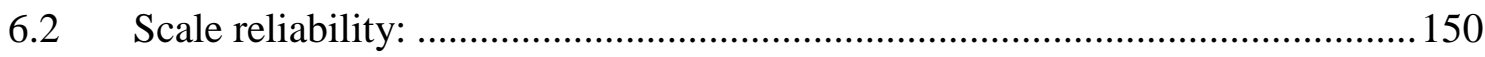

6.3 Descriptive statistics of participants responses ............................................. 151

6.3.1 Overall attitudes towards the implementation of EHRS ............................ 154

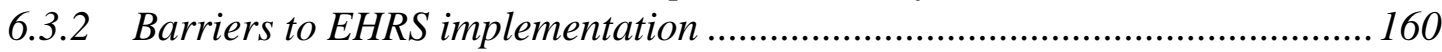

6.3.3 Participant responses to open-ended questions ......................................... 163

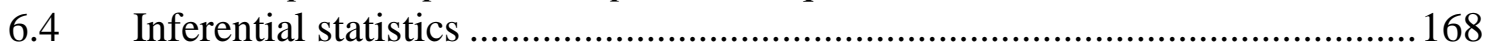

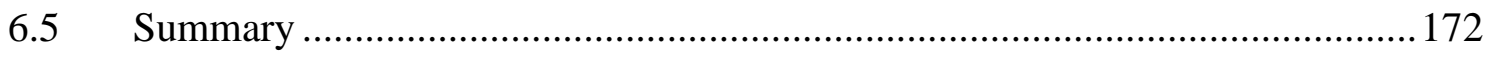

7 Chapter Seven: Study Four: Semi-structured Interviews Exploring the EHRS

implementation in PHCs in SA from a Project Team Perspective...................... 174

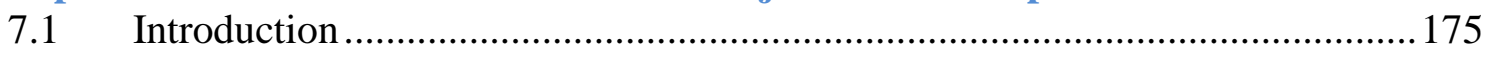

7.2 Theme One: Procedures adopted in the pre-implementation phase.................. 178

7.2.1 Project team selection........................................................................... 178

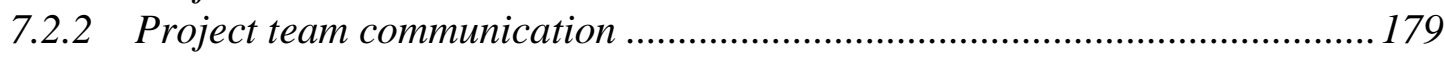

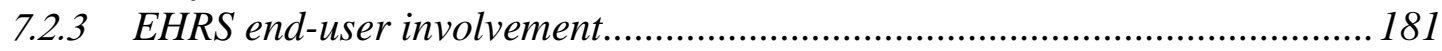

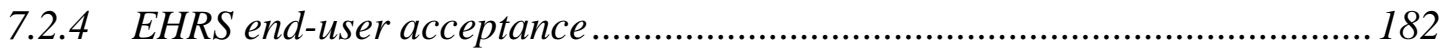

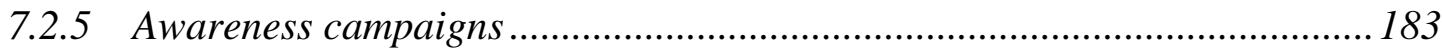

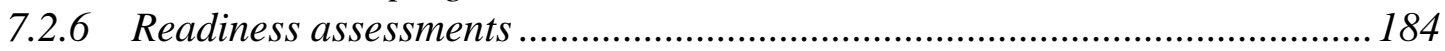

7.2.7 Restructuring and workflow redesign ..................................................... 185

7.3 Theme Two: Plan for EHRS implementation in PHCs.................................... 187

7.3.1 Consultations during the formulation of the EHRS implementation plan .... 187

7.3.2 End-user requirements ................................................................................. 192

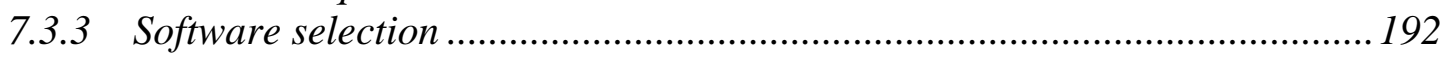

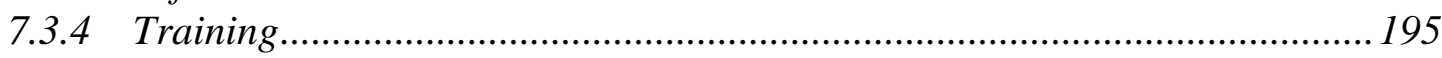

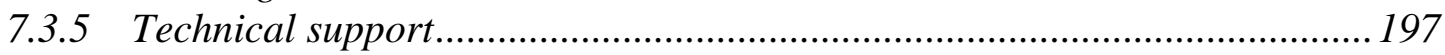

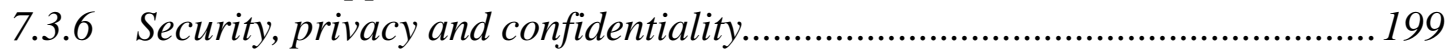

7.4 Theme Three: The role of Centralised Management (CM) ............................202

7.5 Theme Four: The role of Financial Resources (FR) .......................................208

7.6 Theme Five: Facilitators and barriers to EHRS implementation .......................214

7.6.1 Facilitators of EHRS implementation in PHCs in SA................................2 214

7.6.2 Barriers to EHRS implementation in PHCs in SA .......................................2 217

7.6.3 Overcoming barriers............................................................................ 223 


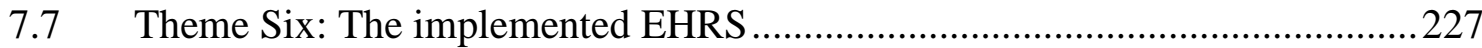

7.7.1 Evaluation of the implemented EHRS .........................................................222

7.7.2 Perceived usefulness of the implemented EHRS ........................................228

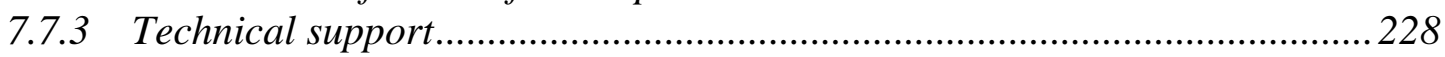

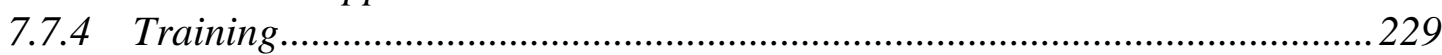

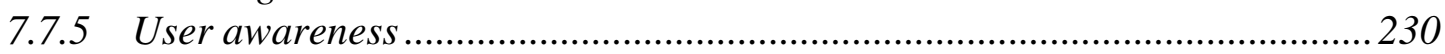

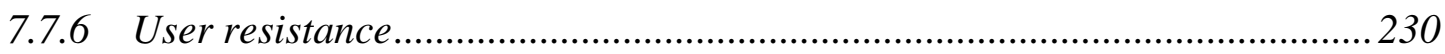

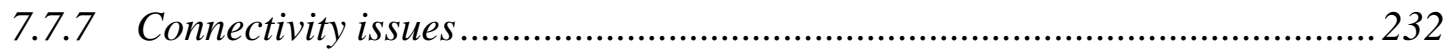

7.7.8 The efficiency of the implemented EHRS ....................................................2232

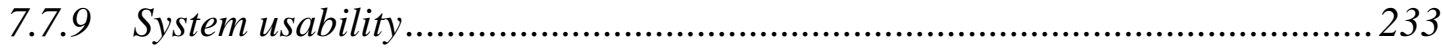

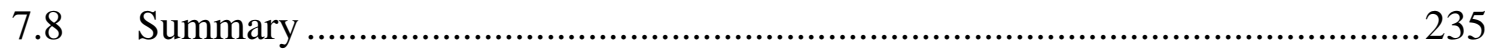

8 Chapter Eight: Discussion ...................................................................... 237

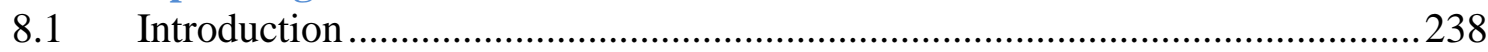

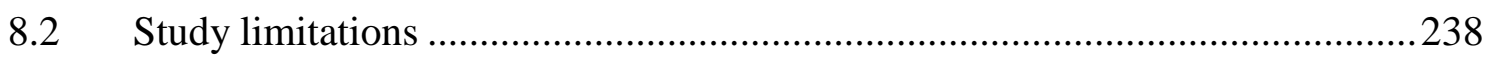

8.3 Readiness of SA PHCs to implement an EHRS .............................................241

8.3.1 PHC readiness for EHRS implementation at the individual level ................ 243

8.3.2 PHC readiness for EHRS implementation at the training and technical

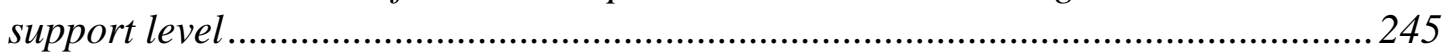

8.3.3 PHC readiness for EHRS implementation at the processes level .................246

8.3.4 PHCs readiness for EHRS implementation at the management structure and administrative and financial support level............................................................ 248

8.3.5 PHC readiness at the technological level ...............................................249

8.4 Facilitators of EHRS implementation in PHCs in SA ….................................250

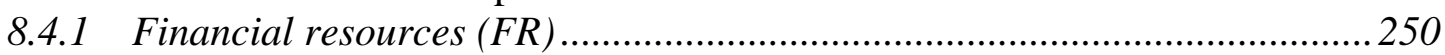

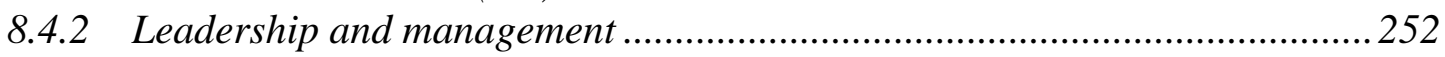

8.4.3 PHC specifications................................................................................. 253

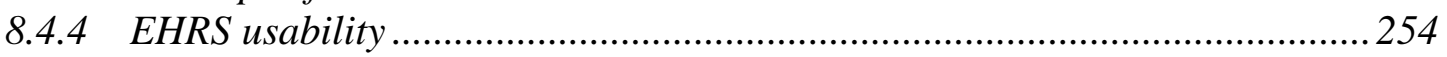

8.4.5 Perceived usefulness of the EHRS and system efficiency.............................25

8.5 Barriers to EHRS implementation in PHCs in SA..........................................256

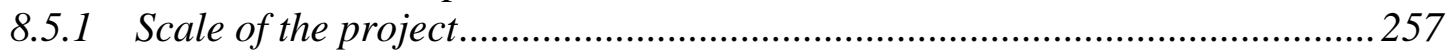

8.5.2 Shortage of experts and changing individuals ............................................2.257

8.5.3 Lack of training and support..................................................................... 258

8.5.4 Geographical challenges and infrastructure issues....................................2260

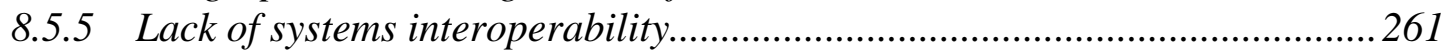

8.5.6 Software selection challenges ................................................................ 262

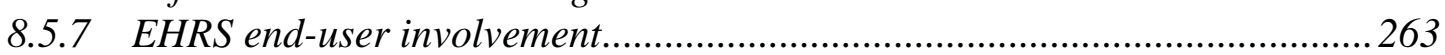

8.5.8 Further recommendations to overcome the above barriers............................ 263

8.6 Overall satisfaction with the EHRS implemented in PHCs in SA.....................264

8.6.1 The impact of EHRS end-user demographic differences on their level of

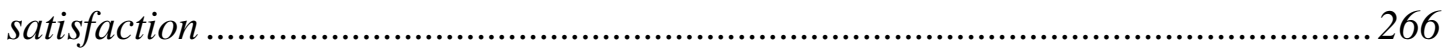

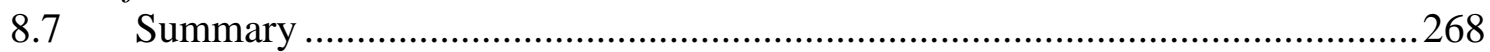

9 Chapter Nine: Conclusion ...................................................................... 270

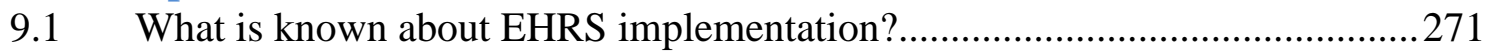

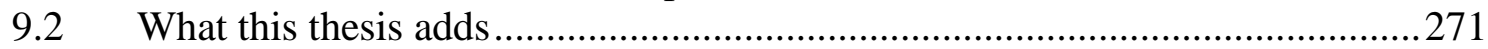

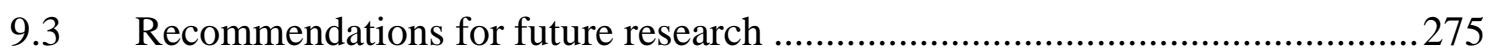

10 References ................................................................................. 276

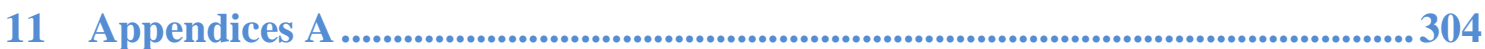

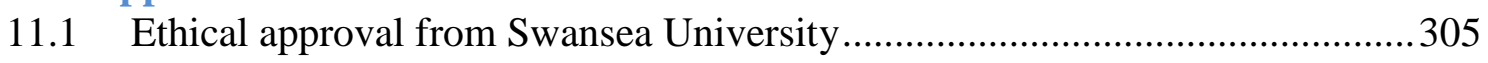




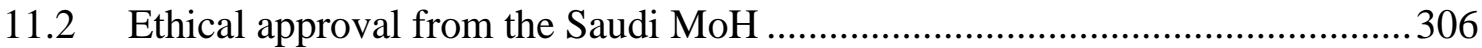

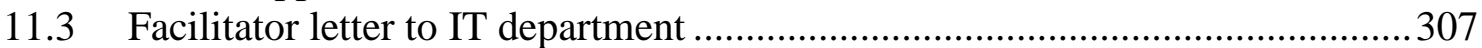

11.4 Facilitator letter to PHCs general management department ..............................308

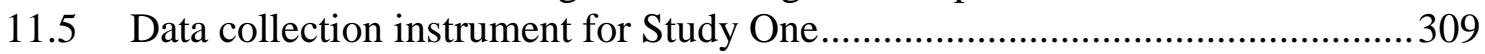

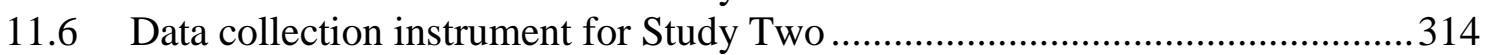

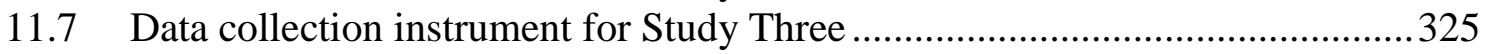

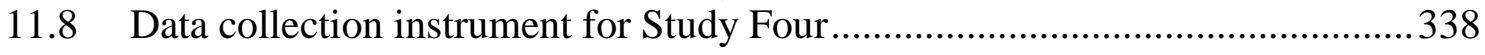

12 Appendices B ............................................................................ 342 


\section{List of Tables}

Table 1.3.1: Top 10 Countries for EHRS Adoption (Robertson, 2013) ............................ 7

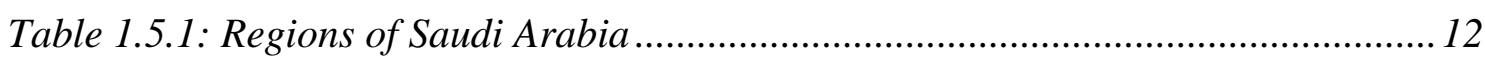

Table 1.5.2: Breakdown of the types of visit to PHCs $(\mathrm{MoH}, 2011 \mathrm{~b})$............................. 14

Table 2.2.1:Keyword combinations used in the literature search strategy .....................20

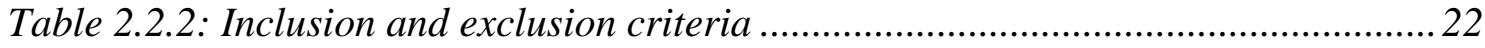

Table 2.3.1: Number of appearances of each included factor....................................... 25

Table 2.4.1: Key and most frequently used papers ........................................................ 27

Table 2.5.1: Factors influencing EHRS implementation at organisational level ........... 29

Table 2.6.1: Factors influencing EHRS implementation at a technological level.......... 44

Table 2.7.1: Factors influencing EHRS implementation at human level........................ 49

Table 3.1.1: Study coverage based on implementation phases.......................................59

Table 3.3.1: Organisational Information Technology/Systems Innovation Readiness

Scale sub-themes.

Table 3.4.1 Main regions in Saudi Arabia and the number of PHCs in each province 73

Table 3.5.1: Regions of Saudi Arabia and number of PHCs in each region .................. 81

Table 3.6.1: Interview guide based on the identified factors from the literature ........... 94

Table 3.6.2: Sample of the tables used to organise quotes for presentation of the final

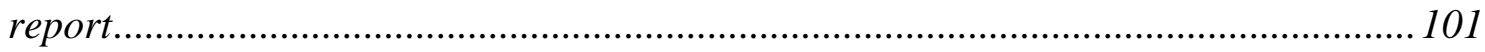

Table 3.6.3:Validity and reliability terms used in quantitative and qualitative research

Table 4.2.1: Cronbach's Alpha reliability measure and the numbers of main scale and sub-scale items

Table 4.3.1: Participant distribution based on gender ............................................. 110

Table 4.3.2:Participant distribution based on previous involvement in EHRS

implementation projects

Table 4.3.3: Participant distribution based on position ............................................ 111

Table 4.3.4: Participant distribution based on role in the implementation project ..... 112

Table 4.3.5: Participant responses to items representing PHC readiness for EHRS

implementation at resources level.

Table 4.3.6: Participant responses to items representing PHC readiness for EHRS implementation at the end-user level. 
Table 4.3.7: Participant responses to items representing PHC readiness for EHRS implementation at the technology level.

Table 4.3.8: Participant responses to items representing PHC readiness for EHRS implementation at the knowledge level.....

Table 4.3.9: Participant responses to items representing PHC readiness for EHRS implementation at the process level

Table 4.3.10: Participant responses to items representing PHC readiness for EHRS implementation at the values and goals level

Table 4.3.11: Participant responses to items representing PHC readiness for EHRS implementation at the management structure level

Table 4.3.12: Participant responses to items representing PHC readiness for EHRS implementation at the administration support level 126

Table 4.3.13: Participant responses to items representing the impact of CM on EHRS implementation in PHCs

Table 4.3.14: Participant responses to items representing the impact of financial resources on EHRS implementation in PHCs

Table 5.2.1: Reliability of benefits and readiness scales ............................................ 133

Table 5.3.1: Participant distribution based on geographical location......................... 134

Table 5.3.2: Participant distribution based on occupation ......................................... 134

Table 5.3.3: Participant distribution based on age .................................................. 135

Table 5.3.4: Participant distribution based on gender.

Table 5.3.5: Participant distribution based on their experience with using a personal computer

Table 5.3.6: Participant distribution based on experience in their current position ... 136 Table 5.3.7: Participant responses to statements regarding PHC staff awareness of the perceived usefulness of an EHRS $(N=13)$. 138

Table 5.3.8: Participant responses regarding the perceived readiness of PHCs to implement an EHRS $(N=13)$ 140

Table 5.3.9: Do you endorse the implementation of an EHRS in PHCs?

Table 5.3.10: Would you be resistant to an EHRS?

Table 5.3.11: Suggestions made by participants regarding recommendations and requirements for successful EHRS implementation. 143

Table 5.4.1: Mann-Witney U test for gender..... 146 
Table 5.4.2: Kruskal-Wallis Test for occupation, age, computer experience, experience at current position, and province differences.

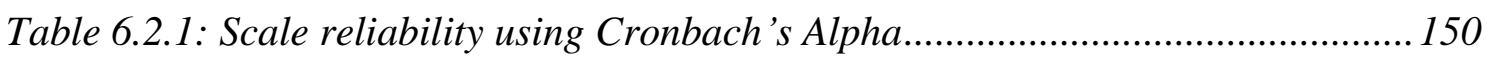

Table 6.3.1: Participant distribution based on geographical location......................... 151

Table 6.3.2: Participant distribution based on gender ............................................. 151

Table 6.3.3: Participant distribution based on occupation ......................................... 152

Table 6.3.4: Distribution of participants based on age .............................................. 152

Table 6.3.5: Participant distribution based on experience in their present position ... 152

Table 6.3.6: Participant distribution based on their experience with using a personal computer.

Table 6.3.7 Participant distribution based on their experience with using an EHRS .. 153

Table 6.3.8: Participant distribution based on current usage of the EHRS in the workplace

Table 6.3.9: Degree of endorsement for each of the seventeen questions relating to perceived usefulness of EHRS

Table 6.3.10: Responses and endorsement to fourteen statements on positive attitudes toward the use of the EHRS scale

Table 6.3.11: Participant responses to eight items reflecting attitudes toward training and support for EHRS 158

Table 6.3.12: Participant responses to seven items reflecting negative attitudes toward the EHRS implemented in PHCs.

Table 6.3.13: Participant responses to sixteen items representing barriers to EHRS implementation.

Table 6.3.14: What are the main things you like about the EHRS?

Table 6.3.15: Do you have any recommendations for the decision-makers to improve the implementation of the EHRS? 166

Table 6.4.1: Mann-Whitney U test for gender and using EHRS at the workplace 168

Table 6.4.2: Kruskal-Wallis test for province, experience in position, experience in using an EHRS, experience in using personal computer, and age

Table 6.4.3: Spearman's correlation coefficient for correlations between the four attitude scales.

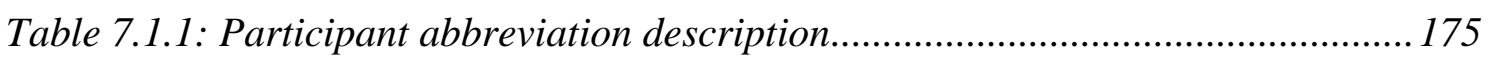

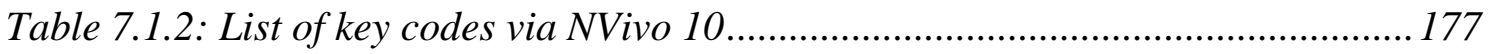

Table 11.8.1: Kruskal-Wallis Test for occupation 342 


\section{List of Figures}

Figure 1.5.1:Comparison of healthcare services in SA between 1987 and 2017.......... 13

Figure 1.6.1: The PHC Solution Roadmap ................................................................. 16

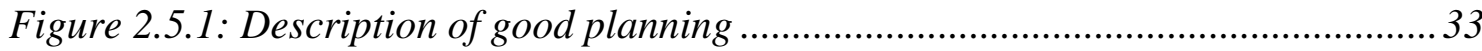

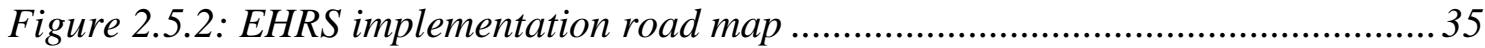

Figure 2.5.3: Factors to be considered during readiness assessments. .......................... 36

Figure 2.5.4: Components of a project leadership role in relation to EHRS

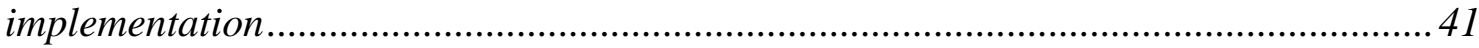

Figure 3.1.1: Methodology and methods employed in the thesis...................................57

Figure 3.1.2: People, Process and Technology Model (PPTM)................................. 58

Figure 3.5.1: The relationship between factors influencing EHRS ............................. 88

Figure 3.6.1: Semi-structured interviews journey. .................................................... 106

Figure 7.1.1: The most frequent words in the interviews ........................................ 176

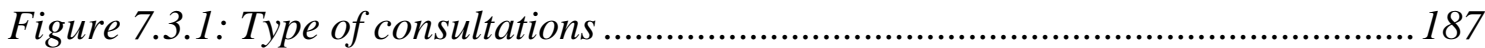




\section{Glossary of Terms}

\begin{tabular}{|c|c|}
\hline Terms & Definitions \\
\hline $\begin{array}{l}\text { Electronic } \\
\text { Health Record } \\
\text { System }\end{array}$ & $\begin{array}{l}\text { "A computerized health information system where providers } \\
\text { record detailed encounter information such as patient } \\
\text { demographics, encounter summaries, medical history, allergies, } \\
\text { intolerances and lab test histories. Some may support order entry, } \\
\text { results management and decision support". (Ludwick \& Doucette, } \\
\text { 2009b p.1-2) }\end{array}$ \\
\hline $\begin{array}{l}\text { Primary } \\
\text { Health Care }\end{array}$ & $\begin{array}{l}\text { "The provision of integrated, accessible health care services by } \\
\text { clinicians accountable for addressing most personal health care } \\
\text { needs, developing a sustained partnership with patients and } \\
\text { practicing in the context of family and community" (WHO, 2013b } \\
\text { p.9). }\end{array}$ \\
\hline $\begin{array}{l}\text { Centralised } \\
\text { Management }\end{array}$ & $\begin{array}{l}\text { "...is the process by which the decision-making authority within and } \\
\text { organisation becomes concentrated in a particular location and/or } \\
\text { group, usually at the top management level" (Ghuman, } 2010 \text { p.275) }\end{array}$ \\
\hline $\begin{array}{l}\text { The } \\
\text { Organisation }\end{array}$ & $\begin{array}{l}\text { "...a social unit (or human grouping) deliberately constructed and } \\
\text { re-constructed to seek specific goals." (Dzimbiri, 2009 p.11) }\end{array}$ \\
\hline $\begin{array}{l}\text { Project } \\
\text { planning }\end{array}$ & $\begin{array}{l}\text { "... a procedural step in project management, where required } \\
\text { documentation is created to ensure successful project completion. } \\
\text { Documentation includes all actions required to define, prepare, } \\
\text { integrate and coordinate additional plans. The project plan clearly } \\
\text { defines how the project is executed, monitored, controlled and } \\
\text { closed." (Techopedia, 2017). }\end{array}$ \\
\hline $\begin{array}{l}\text { A strategic } \\
\text { plan }\end{array}$ & $\begin{array}{l}\text { "...the systematic and organized process whereby an organization } \\
\text { creates a document indicating the way it plans to progress from its } \\
\text { current situation to the desired future situation. It is the set of } \\
\text { decision-making criteria and the decisions taken and implemented } \\
\text { by an organization to definitively and permanently guide its } \\
\text { activities and structure" (Perera \& Peiro, } 2012 \text { p.749) }\end{array}$ \\
\hline Security & $\begin{array}{l}\text { “...procedures, techniques, and technology employed to protect } \\
\text { information from accidental or malicious destruction, alteration, or } \\
\text { access" (Tan, } 2005 \text { p.456). }\end{array}$ \\
\hline Privacy & $\begin{array}{l}\text { “...the right to maintain control over personal information” } \\
\text { (Kayaalp, } 2018 \text { p.9). }\end{array}$ \\
\hline Confidentiality & $\begin{array}{l}\text { “...the responsibility of custodians and recipients of an individual's } \\
\text { health information to use or disclose it only as authorized" (Tan, } \\
2005 \text { p.456). }\end{array}$ \\
\hline $\begin{array}{l}\text { Project } \\
\text { leadership }\end{array}$ & $\begin{array}{l}\text { "... an ability to get things done well through others" (Maley, } 2012 \\
\text { p.377) }\end{array}$ \\
\hline $\begin{array}{l}\text { The Project } \\
\text { Manager }\end{array}$ & $\begin{array}{l}\text { "...person responsible for directing and coordinating the human } \\
\text { effort, material recourses, and findings to produce project } \\
\text { deliverables and achieve the goals and objectives" (Maley, } 2012 \\
\text { p.373). }\end{array}$ \\
\hline
\end{tabular}




\begin{tabular}{|c|c|}
\hline Super users & $\begin{array}{l}\text { "Super users are typically identified as workers who have acquired } \\
\text { sufficient skills to utilize modern day information systems } \\
\text { applications. Super users have the ability to act as healthcare } \\
\text { informatics resources that may reduce dependence on IT } \\
\text { resources" (Boffa \& Pawola, } 2006 \text { p.2). }\end{array}$ \\
\hline Usability & $\begin{array}{l}\text { "...the extent to which a system, product, or service can be used by } \\
\text { specified users to achieve specified goals with effectiveness, } \\
\text { efficiency, and satisfaction in a specified context of use" (ISO, } \\
\text { 2013). }\end{array}$ \\
\hline Ease of use & $\begin{array}{l}\text { "...the degree to which a person believes that using a particular } \\
\text { system would be free of effort" (Davis, } 1989 \mathrm{p} .320) \text {. }\end{array}$ \\
\hline Interoperability & $\begin{array}{l}\text { "...existing between two applications when one application can } \\
\text { accept data (including data in the form of a service request) from } \\
\text { the other and perform the task in an appropriate and satisfactory } \\
\text { manner (as judged by the user of the receiving system) without the } \\
\text { need for extra operator intervention" (ISO, 2001 p.2) }\end{array}$ \\
\hline $\begin{array}{l}\text { User } \\
\text { satisfaction }\end{array}$ & $\begin{array}{l}\text { "...the overall evaluation of a user's experience in using the } \\
\text { system and the potential impact of the system" (Yusof et al., } 2008 \\
\text { p.391). }\end{array}$ \\
\hline $\begin{array}{l}\text { Organisational } \\
\text { factors }\end{array}$ & $\begin{array}{l}\text { Are those resources, procedures and activities occurring in this } \\
\text { social unit or human grouping (Ramanathan, 2009). }\end{array}$ \\
\hline $\begin{array}{l}\text { Qualitative } \\
\text { research }\end{array}$ & $\begin{array}{l}\text { "... a research strategy that usually emphasizes words rather than } \\
\text { quantification in the collection and analysis of data. As a research } \\
\text { strategy it is broadly inductivist, constructionist, and interpretivist, } \\
\text { but qualitative researchers do not always subscribe to all three of } \\
\text { these features" (Bryman, 2012). }\end{array}$ \\
\hline $\begin{array}{l}\text { Quantitative } \\
\text { research }\end{array}$ & $\begin{array}{l}\text { "... a methodology for evaluating objective theories by investigating } \\
\text { the relationship between variables. The variables are the ones that } \\
\text { can be quantified, usually by certain instruments, in order to analyse } \\
\text { the digital data with the aid of statistical methodologies". (Creswell, } \\
2014 \text { p.155) }\end{array}$ \\
\hline $\begin{array}{l}\text { Mixed methods } \\
\text { approach }\end{array}$ & $\begin{array}{l}\text { "... a methodology to conduct enquiry by gathering both qualitative } \\
\text { and quantitative data, combining two types of data, and using unique } \\
\text { designs that might contain philosophical suppositions and } \\
\text { theoretical aspects. The main supposition of this type of inquiry is } \\
\text { that the integration of both qualitative and quantitative } \\
\text { methodologies offers a better and total comprehension of the } \\
\text { research topic than that of offered by each approach individually". } \\
\text { (Creswell, } 2014 \text { p.215) }\end{array}$ \\
\hline $\begin{array}{l}\text { Thematic } \\
\text { analysis }\end{array}$ & $\begin{array}{l}\text { "A method for identifying, analysing and reporting patterns within } \\
\text { data" (Braun \& Clarke, } 2006 \text { p.79). }\end{array}$ \\
\hline $\begin{array}{l}\text { Simple random } \\
\text { sampling }\end{array}$ & $\begin{array}{l}\text { "...is a sampling design in which } n \text { distinct units are selected from } \\
\text { the } n \text { units in the population in such a way that every possible } \\
\text { combination of } n \text { units equally likely to be the sample selected." } \\
\text { (Thompson, } 2012 \text { p.11) }\end{array}$ \\
\hline $\begin{array}{l}\text { Purposive } \\
\text { sampling }\end{array}$ & $\begin{array}{l}\text { "...is non-probability sample to focus on particular characteristics } \\
\text { of population that are of interest, which will best enable the } \\
\text { researcher to answer the research question." (Dhivyadeepa, } 2015 \\
\text { p.105) }\end{array}$ \\
\hline
\end{tabular}




\begin{tabular}{|c|c|}
\hline $\begin{array}{l}\text { Cluster } \\
\text { sampling }\end{array}$ & $\begin{array}{l}\text { "...is a sampling technique where the entire population is divided } \\
\text { into groups or clusters, and a random sample of these clusters are } \\
\text { selected. All units in the selected clusters are included in the sample" } \\
\text { (Dhivyadeepa, } 2015 \text { p.80). }\end{array}$ \\
\hline $\begin{array}{l}\text { Computerised } \\
\text { Physician } \\
\text { Order Entry }\end{array}$ & $\begin{array}{l}\text { "The portion of a clinical information system that enables a } \\
\text { patient's care provider to enter an order for a medication, clinical } \\
\text { laboratory or radiologytest, or procedure. The care provider is most } \\
\text { often a physician, but we would also consider CPOE when a } \\
\text { Physician Assistant (PA) or Nurse Practitioner (NP) with } \\
\text { medication-ordering privileges uses the computer to enter orders. } \\
\text { The system then transmits the order to the appropriate department } \\
\text { or individuals, so it can be carried out. The most advanced systems } \\
\text { also provide real-time clinical decision support, such as dosage and } \\
\text { alternative medication suggestions, duplicate therapy warnings, and } \\
\text { drug-drug interaction checking." (Sittig et al., 2005 p.561). }\end{array}$ \\
\hline $\begin{array}{l}\text { Clinical } \\
\text { Decision } \\
\text { Support System }\end{array}$ & $\begin{array}{l}\text { "Software that designed to be a direct aid to clinical decision- } \\
\text { making, in which the characteristics of an individual patient are } \\
\text { matched to a computerized clinical knowledge base and patient- } \\
\text { specific assessments or recommendations are then presented to the } \\
\text { clinician or the patient for a decision." (Sim et al., 2001 p.528). }\end{array}$ \\
\hline $\begin{array}{l}\text { Project } \\
\text { Champions }\end{array}$ & $\begin{array}{l}\text { "The project champion is the person within an organization } \\
\text { implementing a project who takes on the burden of ensuring } \\
\text { everyone involved is on board and behind the ultimate success of the } \\
\text { project." (Miles, 2013) }\end{array}$ \\
\hline
\end{tabular}


Table of Abbreviations

\begin{tabular}{|l|l|}
\hline Terms & Abbreviations \\
\hline Centrlised Management & CM \\
\hline Financial Resources & FR \\
\hline Chief Executive Officer & CEO \\
\hline Clinical Decision Support System & CDSS \\
\hline Clinical Information System & CIS \\
\hline Clinical Information System Implementation Evaluation Scale & CISIES \\
\hline College of Human and Health Sciences & CHHS \\
\hline College of Medicine & CoM \\
\hline Communications and Information Technology Commission & CITC \\
\hline Computer Assisted Qualitative Data Analysis Software & CAQDAS \\
\hline Computrised Physician Order Entry & CPOE \\
\hline Electronic Health Record System & EHRS \\
\hline Electronic Medical Records & EMR \\
\hline Electronic Patient Record & EPR \\
\hline Electronic Referral System & E-Referral \\
\hline External Research Review Committee & ERRC \\
\hline Health Level Seven & HL7 \\
\hline Heath Informatics & HI \\
\hline Information Communication Technology & ICT \\
\hline Computerised Patient Record System & CPRS \\
\hline Information Technology & IT \\
\hline Institute of Medicine & IOM \\
\hline Ministry of Health & MoH \\
\hline National Electronic Health Record System & NEHRS \\
\hline National Health Service & NHS \\
\hline Organisational Information Technology/Systems Innovation & OITIRS \\
Readiness Scale & \\
\hline Personal Computer & PC \\
\hline Picture Archiving Communication System & PACS \\
\hline Primary Healthcare Centres & PHCs \\
\hline Request for Proposal & RFP \\
\hline Research Ethics Committee & REC \\
\hline Saudi Arabia & SA \\
\hline Saudi Association for Health Informatics & SAHI \\
\hline United Kingdom & UK \\
\hline United States & US \\
\hline & \\
\hline
\end{tabular}




\section{Chapter One: Introduction and Background}


Since the 1960s, Information Communication Technology (ICT) has been responsible for the performance enhancement and improvement of healthcare services (Atherton, 2011; Ortiz et al., 2003). The implementation of Information Technology (IT) in the last few decades of the twentieth century has led to a revolution in the way work is carried out and the way in which information is categorised and documented. The speed and precision that the IT revolution brought about made the governments of developed countries (where this revolution originated) immediately adopt these advanced, fast and efficient systems (Biruk et al., 2014). Moreover, the implementation of the Electronic Health Record System (EHRS) has become a priority for both developed and developing countries (Biruk et al., 2014).

However, Deutsch et al. (2010); Greenhalgh et al. (2009); Lorenzi et al. (2009); Madore et al. (2015); and Smith (2003) have argued that EHRS implementation is very complicated due to the shortage of experience with its implementation and the associated issues. Although, the barriers to EHRS implementation have been described, many of them remain unresolved (Chao et al., 2013). Therefore, it has been suggested that further research and investigation is necessary to overcome these barriers (Chao et al., 2013). According to Greenhalgh et al. (2008); Keshavjee et al. (2006); Lorenzi et al. (2004); and Pare et al. (2008), around fifty percent of EHRS implementation projects around the world have failed. Others have estimated that the proportion of unsuccessful IT projects in the healthcare setting could be as high as seventy percent (Ammenwerth et al., 2006). In addition, according to Gagnon, Desmartis, Labrecque, Legare, et al. (2010), the implementation of EHRS in Primary Healthcare Centres (PHCs) remains a challenge than its implementation in secondary care, such as hospitals.

Across disciplines, at all levels, and throughout the world, it is recognised that the provision of healthcare is becoming ever more complex (Sanchez et al., 2005), particularly in developing countries due to infrastructure issues, organisational workflow issues and cost challenges (Sahay, 2001). Due to this complexity, the introduction of ICT in healthcare organisations poses many challenges (Sahay, 2001). According to Sanchez et al. (2005), one of the obstacles to EHRS implementation is the large number of healthcare practitioners working in these organisations, complicating the process of EHRS implementation. 
Knowledge about the usefulness and benefits of EHRS, as well as the implementation costs and other barriers, is considered scant in developing countries (Were \& Meslin, 2011). Despite the fact that research on the impact of EHRS and its potential benefits has been conducted in developed countries, knowledge about the system's impact is still conflicting (Were \& Meslin, 2011). Moreover, implementation in developing countries requires greater effort than in developed countries, because the readiness of the healthcare organisations is lower with respect to aspects such as IT and infrastructure (Simba \& Mwangu, 2004; WHO, 2007).

As mentioned previously, approximately half of EHRS implementation projects have failed. In Saudi Arabia (SA), the national success level of EHRS implementation is not fully known, as to date there has been no research conducted to evaluate the implementation experience nationally. However, the Saudi Ministry of Health $(\mathrm{MoH})$ has previously attempted to implement EHRS in Primary Health Care (PHC) with limited success. Since no research on EHRS implementation in PHC has been conducted in SA, the current thesis attempts to fill this gap and evaluate the barriers and facilitators to successful implementation. It is hoped that this could help the Saudi MoH and other developing countries to overcome implementation challenges and introduce the best practice when implementing EHRS in PHCs. To realise this goal, the current research aims to explore the large-scale implementation of EHRS in PHCs in SA.

To achieve this aim, the following objectives were defined:

- To undertake a narrative literature review to determine the main factors, facilitators and barriers that influence EHRS implementation, as well as to describe the best practice to implement new EHRS.

- To assess PHC readiness for EHRS implementation through a survey and interviews with project team members and PHC staff.

- To determine the barriers to and facilitators of EHRS implementation in PHCs in SA by interviewing project team members and surveying EHRS end-users.

- To evaluate the impact of Financial Resources (FR) and Centralised Management (CM) on the implementation of EHRS in PHCs in SA.

- To evaluate the implemented EHRS in PHCs, in SA, by surveying EHRS endusers. 


\subsection{Importance of the Study}

The introduction of EHRS in Saudi healthcare organisations is considered to be one of the highest priorities of policy-makers (Hasanain et al., 2014). However, there is currently a dearth of available literature, particularly in the fields of EHRS implementation assessment in the primary care setting in SA. Each country has its own unique and individual culture and systems, and if EHRS implementation is to be successful in the long-term, it is necessary for it to conform to the distinctive customs and traditions of each country (Ash et al., 2007). Despite having some characteristics of a traditional organisation, the healthcare sector is different to other sectors, mostly due to the complexity and social hierarchical structures both within and between institutions (Aarts et al., 2004; Bossen, 2007). Therefore, research carried out in other sectors cannot always be applied directly in the healthcare sector. A thorough examination of the research on EHRS implementation in the healthcare context and from the perspective of the organisation is therefore necessary. This study will therefore investigate EHRS implementation assessment methods and tools that are currently available in the hope that it will contribute to this field. Despite the remarkable growth in the volume of published research determining and defining the facilitators and drivers to EHRS implementation in the past few decades (Lennon et al., 2017; Mair et al., 2012), the published research has focused on secondary care organisations or small-scale projects (Lennon et al., 2017). This proposed research is important due to the limited research that has focused on largescale projects, in particular those related to implementation in PHCs.

Although a number of researchers (e.g. Ash \& Bates, 2005; Hsiao et al., 2011; Podichetty \& Penn, 2004; Sturzlinger et al., 2009) have conducted studies in developed countries, few have been conducted in developing countries (Were \& Meslin, 2011), and specifically in Arab countries (Saleh et al., 2016). The Saudi government has allocated four billion Saudi Riyals (the equivalent of $£ 714$ million) to the establishment of the National Electronic Health Record (NEHR) in the future and for the e-health strategy to be accomplished (Almalki et al., 2011). Currently, more than seventy projects have been identified to achieve this e-health vision $(\mathrm{MoH}, 2013 \mathrm{~b})$. The $\mathrm{MoH}$ of SA plans to implement EHRS in all PHCs following the previous failure of other projects at a cost of billions of Saudi Riyals (MoH, 2013c). According to verbal communication with the director of E-health strategy at the Saudi MoH, previous attempts to implement EHRS in 
PHCs have failed. The decision was made to implement EHRS from the standpoint of investing in such types of projects, and what these projects offer in the long-term. Therefore, it is important to ensure that the likelihood of success is high and that the $\mathrm{MoH}$ considers what will influence successful implementation. Finally, this study is important because it will explore the implementation of the EHRS in two different time periods and will focus on two different projects. Therefore, the findings, which include lessons learned from the failed attempts, will be beneficial to policy makers and project management because, to the best of my knowledge, to date, the new EHRS implementation project in the Saudi PHCs is still in early phases and no EHRS implemented yet.

\subsection{Contribution}

Whilst studies in the field of EHRS have been conducted in many countries, there is no existing research on the implementation of EHRS at PHCs in SA. Moreover, as mentioned earlier in this chapter, each country has its unique system, hence, SA may face a unique challenge and may also benefit from unique facilitators. Each implementation project has its challenges and facilitators. Therefore, the level of success or failure may vary based on the project scale, organisation size and place of implementation (Cresswell et al., 2013; Gagnon, Ouimet, et al., 2010). Causes of failure or success may not be applicable for all types of implementation projects due to this variance. The challenges that hinder the success of EHRS implementations encouraged me to investigate and explore the implementation of large-scale projects, which can be more complicated and may face more challenges. I believe that it would be useful to examine the underlying causes and issues that influence the success of EHRS implementation.

This study contributes to existing knowledge on the subject in several ways. The thesis first examined narrative reviews to determine factors that influence EHRS implementation and acquire broad insight about EHRS implementation as well as a welldefined list of factors that influence it. This thesis then explored the main factors that influence EHRS implementation in particular large-scale projects. In addition, it investigated the barriers and facilitators which impact the success of EHRS implementation in large-scale projects. The determined factors in this thesis, which include newly identified initiatives such as Centralised Management (CM), are 
considered to be useful for researchers and decision-makers alike. Therefore, the newly determined factors in this thesis will provide insight for decision-makers in charge of large-scale projects. This thesis then highlighted a unique experience of large-scale implementation of EHRS in PHCs through exploring multiple perspectives, starting from the top level of management through to project teams and system end-users.

Since this research is fully funded by the Saudi government as part of their development programme to improve healthcare services (Vision 2030), it provides guidance and recommendations based on previous literature, and the findings of this thesis support decision-making concerning the implementation of EHRS in PHCs in SA and other developing countries, in particular the Arab Gulf Countries (AGC). Overall, these findings contribute in several ways to our understanding of the implementation of the EHRS and provide a basis for researchers and policymakers alike.

This thesis also makes a methodological contribution. It presents a systematic methodological framework commensurate with the aim of this thesis. It provides useful tools with which to explore large-scale implementation of EHRS in PHCs by mixing both qualitative and quantitative research. Moreover, the edited data collection instruments may be applied to future research elsewhere in the world, especially where there is a focus on large-scale EHRS implementation. Therefore, the current thesis builds on the strengths of previous research by drawing on a multidimensional assessment of PHC readiness for large-scale EHRS implementation, and then evaluation of large-scale implementation of EHRS in PHCs. 


\subsection{Background Information}

\subsubsection{EHRS adoption rate worldwide}

EHRS reform is fast becoming a priority in developed countries (Car et al., 2008). For instance, at the top of the list, Norway and Netherlands both achieved a 98\% EHRS adoption rate in secondary care, followed by UK and New Zealand with a 97\% adoption rate in hospitals (see Table 1.3.1) (Robertson, 2013). Surprisingly, only 69\% of US hospitals have implemented EHRS, although it has the highest expenditure on EHRS implementation projects (Robertson, 2013; Stone, 2014). Unfortunately, due to a variety of factors influencing the implementation of EHRS, EHRS reform has not received the attention of decision-makers in developing countries (Stansfield et al., 2006).

Table 1.3.1: Top 10 Countries for EHRS Adoption (Robertson, 2013)

\begin{tabular}{|c|c|}
\hline Country & Adoption rate \\
\hline Norway & $98 \%$ \\
\hline Netherlands & $98 \%$ \\
\hline United Kingdom & $97 \%$ \\
\hline New Zealand & $97 \%$ \\
\hline Australia & $92 \%$ \\
\hline Germany & $82 \%$ \\
\hline United States & $69 \%$ \\
\hline France & $67 \%$ \\
\hline Canada & $56 \%$ \\
\hline Switzerland & $41 \%$ \\
\hline
\end{tabular}

\subsubsection{Electronic Health Record System (EHRS)}

Early research in Health Informatics (HI) has emphasised that the implementation of clinical computing should be the primary goal in order to enhance patient safety and care quality (Berner et al., 2005). This idea came into existence in the early part of the 1960s and is still being proposed with the modifications, such as technological advancements. 
Such modifications will simplify the procedure so that patient care can be provided accurately and on time (Atherton, 2011).

In the 1970s, two separate types of EHRS applications were developed. The first made use of an integrated design concept, where an entire set of applications would be supported by a single, large shared computer. The second made use of a multi-computer system. However, this was found to be unavailable until future technological advancements in networking systems were made (Shortliffe et al., 1990). Many of the technologies developed during that era in order to help facilitate the diagnosis of illnesses in clinics, however, acted as stand-alone systems and were not integrated into the Clinical Information System (CIS) of hospitals. Atherton (2011) reported that two forms of EHRS applications were implemented in the 1970s: Decentralised Hospital Computer Program (DHCP) and Computerised Patient Record System (CPRS).

Since the 1980s, HI has shifted towards computerisation of hospital administration and financial aspects (Amatayakul, 2007; Martikainen et al., 2012). This process of computerisation has been found to be straightforward, simple, automated and also economical. With the advancement and enhancement of clinical systems, secondary areas were also being computerised. The typical secondary areas becoming computerised are laboratories, radiology departments and pharmacy departments (Amatayakul, 2007). According to Gagnon, Desmartis, Labrecque, Légaré, et al. (2010), the first use of EHRS in PHCs was in the 1990s.

\subsubsection{EHRS applications and functions}

According to DesRoches et al. (2008), EHRS can be classified into two levels, as below:

- Basic system: This includes data such as patient demographics, medication data, patient issues and clinic notes, as well as basic orders such as laboratory tests and $\mathrm{x}$-rays

- Fully Functioning System: This includes a basic data system and also a record of the medical history in its current state. Other data includes prescription entries, laboratory and radiology tests, electronic prescriptions, electronic images and electronic orders, clinical decisions that warn about drug interactions or contra- 
interactions and reminders about guidelines that relate to screening and intervention.

Various names and acronyms are used interchangeably to describe EHRS, and include: Electronic Patient Record (EPR), Electronic Referral System (E-Referral), Electronic Medical Record (EMR), Clinical Decision Support System (CDSS), Electronic Prescriptions System (E- Prescriptions), and Computerised Physician Order Entry (CPOE). Those applications can be integrated together as tools within one system to be installed in healthcare organisations.

If the benefits of EHRS on healthcare systems are to be realised, then its functions must be understood. According to a statement by the Institute of Medicine in 2003, the function of EHRS is to improve particular aspects such as patient safety, effective delivery of patient care, chronic illnesses management, and efficiency (IOM, 2003). The core functions can be categorised into seven groups: health data, management actions, decision support, requests entry, patient support, public health and electronic communication (IOM, 2003).

\subsubsection{The importance of EHRS}

The positive impact of EHRS was noticed almost immediately in the field of primary healthcare (Holroyd-Leduc et al., 2011). It has been suggested that implementing EHRS will enhance the accessibility and the process of distribution of health records between authorised users (Ahmadian et al., 2015; Barrows \& Clayton, 1996; Gagnon et al., 2016; Sicotte \& Pare, 2010; Weiskopf \& Weng, 2013). In addition, EHRS can improve the economic and administrative abilities of all healthcare institutions and, also, directly influence the quality of care provided to patients (Ash et al., 2003; Gagnon, Nsangou, et al., 2014; Murray et al., 2010; Xierali et al., 2013; Yontz et al., 2015).

Acting as an electronic version of paper medical records, EHRS are considered to be an essential part of healthcare provision in today's information systems era (de la Torre et al., 2012). EHRS have been shown to play a vital role in elevating healthcare to the next level and ensuring an enhanced degree of healthcare (Harman et al., 2012; Wang et al., 2005). EHRS improves not only the quality of care being provided, but also can improve 
patient safety (Gascon et al., 2013; Kannoju et al., 2010) and allow the patient to be managed more efficiently and accurately (Niazkhani et al., 2009).

\subsection{Primary Healthcare Centres}

As part of a country's healthcare system, PHCs have attained paramount significance in the process of giving care. This fundamental care is critical to the proper delivery of healthcare (Starfield, 1998). Its importance lies in the fact that the PHCs form the primary point of supervision and are the foremost channel for the provision of medical care to a considerable percentage of the population (Saad, 2004). To that effect, PHCs are recognised more and more as substantial alternatives to hospitals, considering firstly that the elderly proportion of the population is growing phenomenally, and secondly that patient autonomy and independence is growing (Calnan et al., 1994). PHCs are a realistic channel for realising quality fundamental medical care, which should be comprehensive, publicly available and easily accessed by the general population (Littlewood \& Yousuf, 2000). In most countries, PHCs are where patients engage with their health system (Gosden et al., 2000). Primary Care Physicians (PCPs), who are also known as Family Physicians (FPs) or General Practitioners (GPs), have the broad medical knowledge necessary to deal with the range of health problems, presented by patients on a daily basis. Generally, face to face visits between patients and physicians in primary care are short, lasting six minutes to one hour (Crampton et al., 2007; Gottschalk \& Flocke, 2005; Middleton et al., 2005) .

An ideal healthcare setting has a twofold agenda: to hinder the advancement of disease by means of prophylaxis, and to alter one's way of living in order to lessen the risk of disease. The basic features of this blueprint include programmes related to maternal and child nutrition, participation by the local community and programmes for immunisation (Littlewood \& Yousuf, 2000). Good primary care is widely believed to be necessary for an effective, efficient, equitable health system and to lead to better health amongst the population (Starfield et al., 2005). Primary care comprises the most basic premises for care, and it remains the fundamental setting for care for most people (Gosden et al., 2000; Green et al., 2001). 


\subsection{The healthcare system in Saudi Arabia}

In 2013, the SA MoH stated that EHRS would be implemented in the country $(\mathrm{MoH}$, 2013b). Different countries use different frameworks for managing their healthcare. This framework can differ from place to place within the same country, depending on the local geography and local government. Furthermore, differences may be identified over time with onward progress and subsequent changes that take place in managing healthcare (AlYousuf et al., 2002). In keeping with modern times and the changing requirements of its citizens, several modifications have already taken place in the way healthcare is managed in SA (Almalki et al., 2011).

The Saudi MoH manages, supervises, controls, operates and finances the health sector (WHO, 2013a). The $\mathrm{MoH}$ is the principal administrative organisation that has been delegated the responsibility of overseeing prophylactic, remedial and rehabilitation functions in healthcare. It proposes to achieve these objectives through key planning, developing particular guidelines related to healthcare, imparting all-inclusive medical assistance and keeping a check on and control over all enterprises connected to healthcare (Alkraiji et al., 2013). The MoH operates via a three-level medical care management system. The primary level consists of health centres, the secondary level consists of general hospitals, and specialist hospitals are at the tertiary stage. All ideas and projects formulated by the $\mathrm{MoH}$ are carried out through this hierarchy. The $\mathrm{MoH}$ operates and supervises all healthcare organisations via its thirteen varied health affairs (see Table 1.5.1) $(\mathrm{MoH}, 2012)$. The current management utilised by the Saudi $\mathrm{MoH}$ (see Section 1.5.2), undermines the role of the health affairs in each region due to less authority provided to those affairs. The role of these health affairs is to act as a communication channel between healthcare organisations (e.g., hospitals and PHCs) and the headquarters of the $\mathrm{MoH}$ and provide direct supervision to all healthcare organisations within those regions. 
Table 1.5.1: Regions of Saudi Arabia

\begin{tabular}{|c|c|}
\hline NO & Name of Region \\
\hline $\mathbf{1}$ & Riyadh \\
\hline 2 & Gassim \\
\hline 3 & Makkah \\
\hline $\mathbf{4}$ & Almadinah \\
\hline $\mathbf{5}$ & Alsharqiah \\
\hline $\mathbf{6}$ & Albaha \\
\hline $\mathbf{7}$ & Asir \\
\hline $\mathbf{8}$ & Najran \\
\hline $\mathbf{9}$ & Hail \\
\hline $\mathbf{1 0}$ & Alshamaliyah \\
\hline $\mathbf{1 1}$ & Jazan \\
\hline $\mathbf{1 2}$ & Tabuk \\
\hline $\mathbf{1 3}$ & Aljouf \\
\hline
\end{tabular}

The $\mathrm{MoH}$ is not the sole provider of care within SA, there are two other healthcare providers: private healthcare providers, and alternate public governmental care providers. The latter includes organisations such as university hospitals and medical care providers, affiliated with the National Guard and Armed Forces. Fifty eight percent of the total healthcare provision is supplied by the $\mathrm{MoH}$, whilst the remainder is provided by the other governmental providers (23\%) and private agencies (19\%) (Alkraiji et al., 2013). Carrying out this exhaustive range of functions costs the Saudi government 39.8 billion Riyals ( $£ 7.9$ billion), which is $6.9 \%$ of the total annual budget (MoH, 2011b).

By 1987, there were 149 hospitals in SA, with more than 26,000 beds in 1,480 clinics, where over 4,000 physicians and 30,000 other staff worked (Tumulty, 2001). Today, 420 hospitals function in the Kingdom, of which 251 are public hospitals, 130 are private hospitals and 39 are non-MOH hospitals. The total beds in all the hospitals number 58,696 , out of which MoH hospitals have 34,450 , which is $58.7 \%$ of all beds in SA. There is roughly one bed for every 483 people in the country, and 20.68 beds for every 10,000 . Today, 197,672 employees work in the healthcare sector in the Kingdom of SA. There 
are 69,226 doctors (of which $22.4 \%$ are native), and 134,632 nurses (of which local Saudis comprise $33.6 \%$ ). In addition, there are estimated to be 15,043 pharmacists working in SA, while the total number of allied health personnel is 78,823 , of which $69.1 \%$ are Saudi (see Figure 1.5.1) (MoH, 2011b).

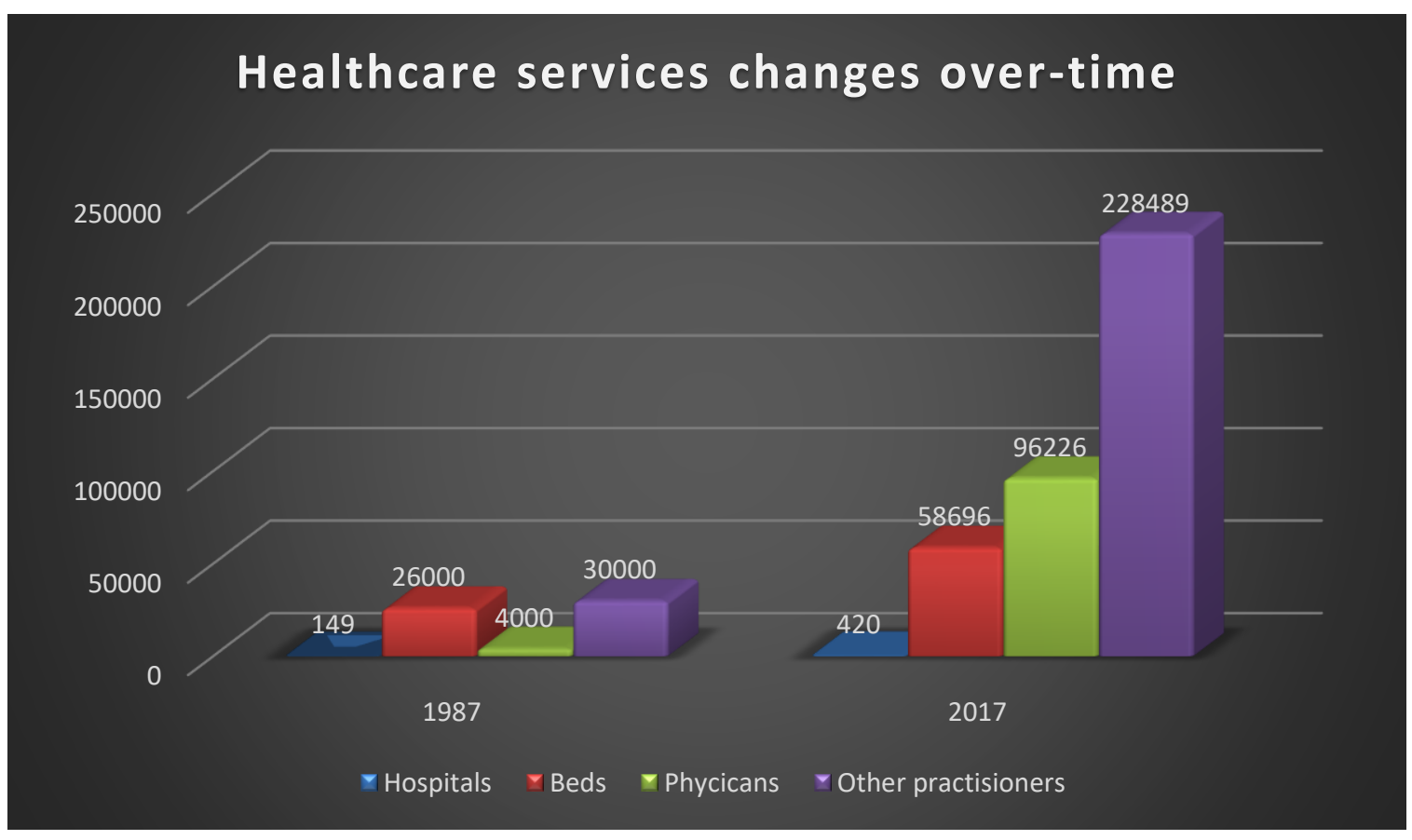

Figure 1.5.1:Comparison of healthcare services in SA between 1987 and 2017

\subsubsection{PHCs in Saudi Arabia}

As part of its duties, the MoH supervises more than 2,109 PHCs divided amongst thirteen regions in Saudi Arabia (see Table 1.5.1). Each PHC provides health services for an estimated 13,455 people (MoH, 2011b). Additionally, there are 1,600 PHCs under construction as part of King Abdullah's project to build 2,000 PHCs. This project is estimated to be costing more than seven billion Riyals (1.25 billion GBP).

In 2002, in the Kingdom of SA, 98 million appointments to see a healthcare professional were made at various medical care facilities. These included 53.5 million visits made to PHCs (Saad, 2004). In 2011, in their statistical report, the MoH disclosed that out of 132 million appointments made at all medical care centres, 54.52 million were at PHCs run for the general public. Typically, every PHC centre has 25,849 visits per year (MoH, 
2011b). A breakdown of the visits to PHCs in Saudi Arabia is given below (see Table 1.5.2):

Table 1.5.2: Breakdown of the types of visit to PHCs $(\mathrm{MoH}, 2011 \mathrm{~b})$

\begin{tabular}{|c|c|}
\hline Type of clinic & Number of visits (million) \\
\hline General & 33 \\
\hline Chronic & 4.7 \\
\hline Dental & 4.7 \\
\hline Antenatal & 0.7 \\
\hline Well-baby clinic & 3.1 \\
\hline Other clinics & 10.1 \\
\hline
\end{tabular}

\subsubsection{Centralised Management (CM) in SA healthcare}

It has been suggested that such a management concept might not be capable of meeting the requirements of the population's future healthcare plans and strategies (Almalki et al., 2011). However, it can become capable if the steps of implementation are well planned, separate multiple roles, and distribute authorities and powers to lower level management. A possible solution can be offering more control and power to the regional directorates at the health affairs in each region (Almalki et al., 2011). According to Alghamdi and Urden (2016), the Saudi MoH is leading and supervising its healthcare sectors (PHCs and hospitals) in a purely centralised manner. It is responsible for formulating plans, legislation of regulations and policies, and managing and providing financial resources. Ekvall (1996) argued that such CM has several disadvantages such as delaying projects, resistance to change, and a decrease in the level of innovation within the organisation. This is thought to be due to fact that the major decisions are being made by very few people (Ekvall, 1996). However, a very recent study revealed that CM contribute to the consistency of procedures and quality of any organisation (Alhamad \& Aladwan, 2019).

\subsection{ICT development in the SA healthcare system}

The healthcare sector of SA lags behind that of developed countries with respect to ICT development (Hasanain et al., 2014). The first appearance of ICT in a healthcare 
organisation in SA was in 1988 (Hasanain et al., 2014). Each healthcare provider is at dissimilar levels of completion in terms of the implementation of these ICTs (Alkraiji et al., 2013), leading to fears that EHRS may not be used to their fullest extent in SA (Almalki et al., 2011). Budgetary constraints and a shortage of medical professionals are some of the primary causes of a lack of adequate IT infrastructure in most Saudi MoH hospitals, as well as private clinics and hospitals. The majority of EHRS in the private sector are more concerned with profit-making, and to that end they lay more emphasis on adopting and implementing accounting software. Only a few governmental healthcare organisations have implemented a new EHRS (Altuwaijri, 2008). According to recent study, the adoption rate of EHRS in Saudi hospitals is not well documented (Aldosari, 2014). However, there are a few initiatives to determine the number of hospitals in SA that have implemented EHRS. For instance, a study conducted in the eastern province of SA noted that only three out of nineteen hospitals had implemented EHRS (Bah et al., 2011). To my best knowledge, currently, the majority of Saudi governmental hospitals have implemented an EHRS. Previous research has attempted to evaluate the level of adoption of EHRS in SA, but concluded that there was no accurate data to determine the number of healthcare organisations that had implemented EHRS (Alsahafi, 2012).

Recently, however, there has been a gradual transformation to electronic transaction. The SA government is recognising the need to invest extensively in e-health (Altwaijiri \& Aldosari, 2008). In order to achieve this, there is a multi-phase proposal to re-develop the ICT sector. This has been planned in four phases with strengthened national plans and initiatives and is to be realised by means of mutual collaboration. Agencies, such as the Communications and Information Technology Commission (CITC), are scheduled to play a primary role in the whole process (CITC, 2005). To ascertain the financial feasibility of this healthcare e-governance project, the MoH undertook a detailed study. One of the favourable conclusions of a study by Qurban and Austria (2008), was that a saving of ten to fifteen percent of the yearly budget for health could be made if the ehealth project was adopted. Another conclusion was that common medical insurance would accrue benefits from adopting the personal smart card. As a result of these positive results, it has been suggested that implementation of EHRS or e-health in SA remains a necessary undertaking, particularly if progress in the field of improving healthcare provision is to be made (Qurban \& Austria, 2008). 
The project for the digital transformation of the 3,600 plus PHCs is projected to take several years (Alkraiji et al., 2013). The automation of PHCs in SA is part of the Saudi e-health strategy (MoH, 2011a). According to (MoH, 2011a), 2600 PHCs will be automated by the implementation of the EHRS. Three different vendors will take the role in the implementation of the Health Information Systems (HISs) in PHCs over ten years (see Figure 1.6.1) (MoH, 2013c) . It should be noted, however, that previous attempts to implement an EHRS in Saudi PHCs have failed (see Section 1.1).

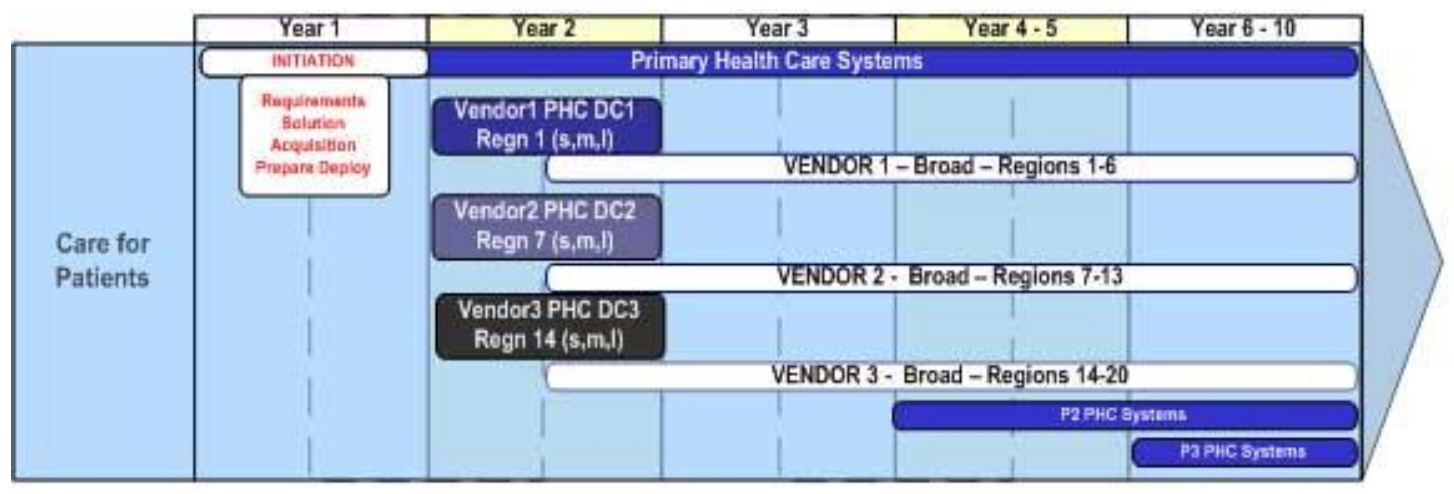

Figure 1.6.1: The PHC Solution Roadmap

To meet the stringent needs of the Saudi healthcare system and enhance healthcare quality, the $\mathrm{MoH}$ has introduced a national level strategy on healthcare services. The Council of Ministers approved this strategy in April 2009. The strategy indicates diversification of funding resources; enhancing information systems, advancing the human workforce and, starting and monitoring the participation of the $\mathrm{MoH}$ in health services. The strategy also motivates the private sector to play an active role in offering health services, enhancing the standards of curative, rehabilitative and preventive care and providing an equal share of services to all parts of the country (Almalki et al., 2011). This strategy, developed for healthcare services, needs to be installed by the $\mathrm{MoH}$, along with several healthcare providers, and will be overseen by the council of health services. The implementation of EHRS is part of the adopted strategy, which will then facilitate the achievement of successful NEHR. The following chapter describes a narrative review of the literature to identify the main factors that may influence the implementation of EHRS, as well as to determine the main barriers and facilitators to the success of the implementation projects. 


\section{Chapter Two: Literature Review}




\subsection{Introduction}

As stated in the previous chapter, the aim of this thesis was to explore the implementation of an EHRS in PHCs in SA. To achieve this aim, I searched and critically appraised the literature in order to identify the main factors that influenced EHRS implementation. This chapter describes the rigorous literature review undertaken and discusses the relevant factors identified by the literature. My aim with this literature review was to obtain a better understanding of the barriers and obstacles that may hinder the success of EHRS implementation, as well as the facilitators that may assist in enhancing its success. I utilised the findings from the literature review to inform the development of the data collection instruments for subsequent parts of the research (interviews and questionnaires).

\subsection{Literature review search strategy}

A narrative literature review was designed to achieve the aims outlined above. Narrative literature review found to be useful to address broad research questions. Therefore, the decision to undertake a narrative literature review was taken as the aim of this review was to obtain a broad overview of EHRS implementation (Kysh, 2013). Through the utilisation of narrative literature review, I was able to determine the main factors influence the EHRS implementation, determine barriers and facilitators to the implementation and then describe the best practice in EHRS implementation. Narrative literature review is not restricted to specific context or specific region which can contribute to reduce any potential bias. Therefore, studies conducted on both secondary and primary care were included. In addition, studies conducted on developed and developing countries were also included (will be explored in detail in the following sections). It was also felt that a narrative literature review would be more appropriate to qualitatively summarising information about particular topic (Kysh, 2013).. The narrative review was rigorously undertaken in four main steps: 1) identification, 2) screening, 3) eligibility, and 4) selection of articles for inclusion (Moher et al., 2009). 


\subsubsection{Identification}

I firstly searched for articles from four key databases within the field of health informatics to ensure that all relevant papers were identified. Four databases were used so as to reduce bias: Medline, Web of Science, and Cumulative Index to Nursing and Allied Health Literature (CINAHL). According to Bahaadinbeigy et al. (2010), CINAHL, EMBASE and Medline are the most appropriate databases for those who are planning to undertake research in the field of health services. They suggested using these different databases to ensure all relevant research is identified. However, Web of Science was searched instead of EMBASE due to its relevance, as suggested by the librarians in Swansea University (SU, 2019). Bahaadinbeigy et al. (2010) found that using two databases only may lead to a loss of at least $18 \%$ of required studies. Therefore, I performed the same search strategy using more than two databases. Furthermore, I searched using multiple keywords and their synonyms (see Table 2.2.1). Although this variety may lead to the identification of a larger number of articles, it assists in reducing bias. The full text versions of some of the articles were not fully accessible in the used databases. The full texts of these articles were therefore downloaded from other databases, including Wiley, Health Affairs, Springer, ScienceDirect, SAGE, Saudi Digital Library and Swansea University's library. Following retrieval of the full text articles, the reference list of key relevant articles (in particular, systematic reviews) were screened to identify any further relevant literature that did not appear in the initial search. Other resources were searched to identify grey literature, websites, conference proceedings, Masters and Ph.D. theses.

All searches were limited to resources in the English language and publication between the years of 2000 to 2018 . The decision to limit the search to this timeframe was made based on the fact that health informatics is a relatively new field that has seen substantial growth in the last two decade. Therefore, all papers published before 2000 were excluded from the search. I used relevant key words to identify the published papers (see Table 2.2.1). The keywords were divided into two groups. One group included EHRS and their application as well as other potential terms that referred to technology in healthcare. The second group included issues around the processes surrounding EHRS, such as implementation and adoption. Keywords within each group were combined using the Boolean precedence, OR, and then the two groups were combined using the Boolean precedence, AND (see Table 2.2.1) 
Table 2.2.1:Keyword combinations used in the literature search strategy

\begin{tabular}{|c|c|c|}
\hline Group 1 & AND & Group 2 \\
\hline Electronic Health Record & & Adoption \\
\hline OR & & OR \\
\hline Electronic Medical Record & & Implementation \\
\hline OR & & OR \\
\hline Electronic Patient Record & & Facilitators \\
\hline OR & & OR \\
\hline Clinical Information System & & Barriers \\
\hline OR & & OR \\
\hline Health Information System & & Factors \\
\hline OR & & OR \\
\hline E-prescribing & & Readiness \\
\hline OR & & OR \\
\hline Picture Archiving and Communication System & & Attitude \\
\hline OR & & OR \\
\hline E-referral & & Satisfaction \\
\hline OR & & OR \\
\hline Clinical Decision Support System & & Resistance \\
\hline OR & & OR \\
\hline Computerised Physician Order Entry & & Acceptance \\
\hline OR & & OR \\
\hline \multirow[t]{3}{*}{ Information Communication Technology } & & Perspective \\
\hline & & OR \\
\hline & & Perception \\
\hline
\end{tabular}




\subsubsection{Screening}

Once the search of the selected databases to find the most relevant literature in the field of the EHRS implementation had been performed, inclusion and exclusion criteria were applied to identify the literature most relevant to the research question. The search employed in Section 2.2.1 returned 1,979 papers. All 1,979 papers were imported into the reference manager software, Endnote, which helped to detect any duplication. Of the 1,979 papers identified, 852 references were deleted due to duplication. All remaining references were reviewed to determine eligibility for inclusion. Following an initial title review, 637 articles were rejected. A further 244 were rejected following an abstract review. Following a full text review, 170 articles were excluded, giving a final number of included articles of 76 (see Section 2.2.3). The full text of three of the papers were not obtainable.

\subsubsection{Eligibilitity}

Several inclusion and exclusion criteria were adopted in selecting relevant papers for this literature review (see Table 2.2.2). The primary focus of the literature review was to identify the main factors that influenced EHRS implementation, as well as the barriers to and facilitators of implementation projects. Although the literature search was not limited to PHCs, the secondary focus was on research conducted in PHCs. Research conducted in secondary care and other health organisations was therefore included where relevant, but the priority was on research conducted in PHCs. Given the volume of literature available, in order to give an overview of the main themes, the literature review focused mainly on systematic and literature review articles and meta-analyses. In addition, papers on best practice for EHRS implementation which included the process of the implementation were included. Papers that focused on the implementation of other electronic systems within healthcare, such as CPOE, CDSS, E-referral and PACS were also included, as it was felt that implementation issues with these systems may also be relevant to EHRS. Research from both developed and developing countries were included in the literature review. Although, no quality assessment was performed to the included studies, the priority was for peer-reviewed studies. Therefore, most of included studies in this narrative literature review were peer-review studies. Some of non-peer-reviewed 
papers were also included due to their relevance to the aim of this literature review. However, the selection of both peer-reviewed and non-peer-reviewed were based on several criteria such as clarity and completeness (La Torre et al., 2015)

Table 2.2.2: Inclusion and exclusion criteria

\begin{tabular}{|l|l|}
\hline Inclusion & Exclusion \\
\hline Published between 2000-2018 & Published before 2000 \\
\hline Both secondary care and primary care & Impact of EHRS \\
\hline Developed and developing countries & Secondary use of clinical data \\
\hline $\begin{array}{l}\text { Large and small-scale projects } \\
\text { Implementation of the EHRS and its }\end{array}$ & Patient perspective \\
\hline English language & Paper-based health records \\
\hline All methods & Focus on tele-health and tele-medicine \\
\hline All population & \\
\hline
\end{tabular}

On the other hand, several exclusion criteria were employed to assist in obtaining the most relevant research. Firstly, research focused on EHRS benefits, advantages, meaningful use and impact on healthcare were excluded, because this thesis was not aiming to evaluate the impact of an EHRS on healthcare services. Moreover, I excluded studies focussing on the secondary use of clinical data. I also excluded articles focused on Patient Health Records (PHRs) which evaluated patient access to their own records, because this thesis was not aiming to evaluate patient perspectives toward EHRS implementation. In addition, I excluded articles focussed on health applications, technologies and functions other than those listed in Table 2.2.2, such as tele-medicine, tele-health and speech recognition, as these technologies are different from the EHRS. Finally, research conducted to explore or evaluate paper and traditional patient records were also excluded, as this thesis focuses on EHRS and its applications only. 


\subsubsection{Inclusion and exclusion flow diagram}

A total of 76 articles were included in the literature review after utilisation of the exclusion criteria mentioned above.

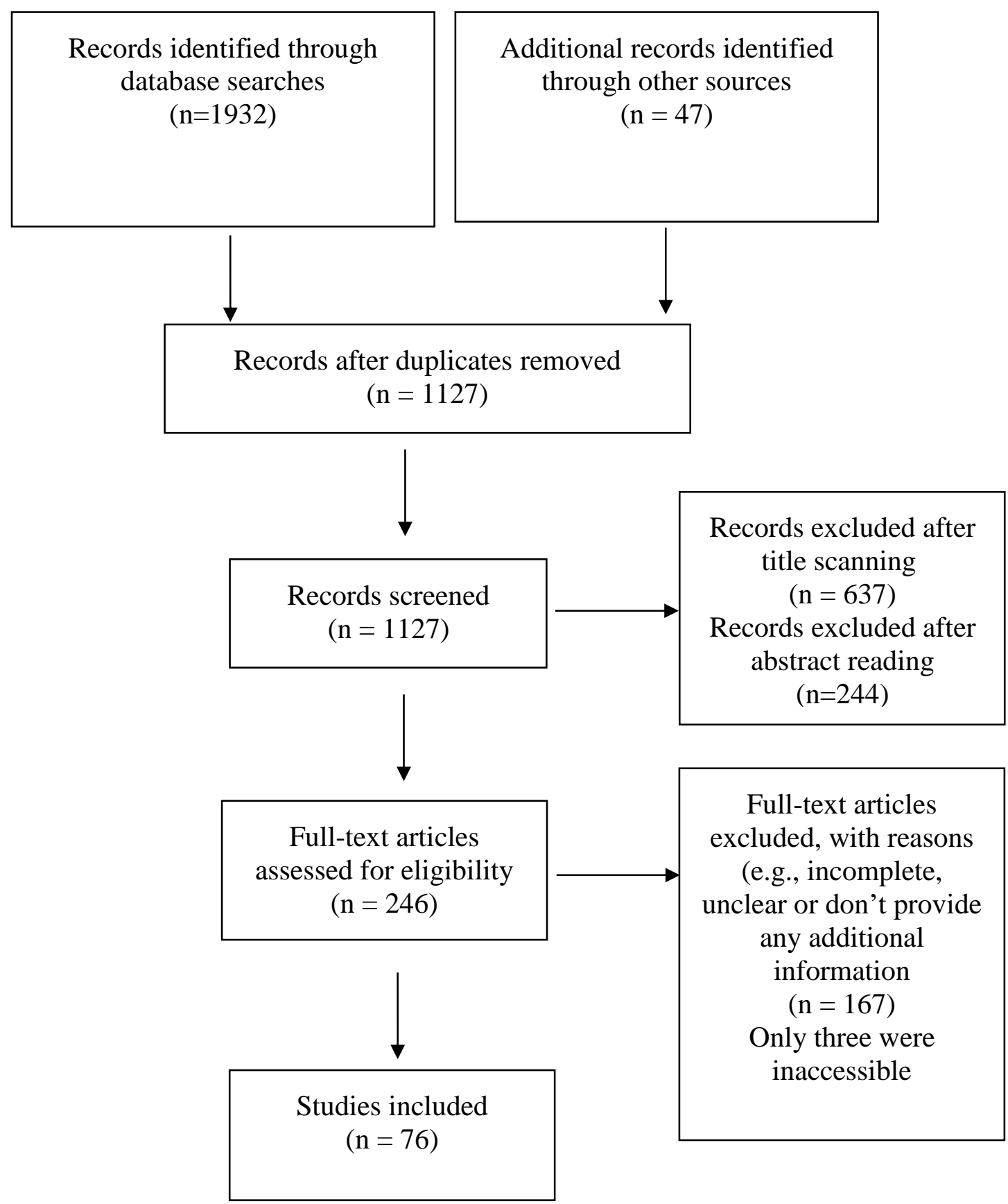




\subsection{Identification of factors}

The qualitative software, NVivo, was used to determine the relevant factors that influenced the EHRS implementation. Firstly, once all relevant papers were selected after using the defined inclusion and exclusion criteria mentioned in the previous section, PDF versions of the papers were imported into the software in order to analyse the content. All imported PDFs were then searched for factors that influenced EHRS implementation. I then highlighted the detected factors in the inserted PDF files. This process was carried out by reading through the PDFs line by line to code and identify all the factors that influenced EHRS implementation. I started with the primary studies, including systematic review papers (e.g. Ash et al., 2012; Gagnon et al., 2012; Keshavjee et al., 2006; Lorenzi et al., 2009; Ludwick \& Doucette, 2009b; Yusof et al., 2008). Primary studies consisted of systematic reviews and the most relevant research that was conducted to address similar research questions. The highlighted factors were then labelled and initial codes (parent nodes) were created in NVivo. Similar statements were highlighted from imported PDFs then linked to the appropriate code.

Reading through individual papers of these primary studies facilitated the determination of most of the included factors in this literature review. Although the majority of factors were identified through the primary studies, I scanned other studies that meet the final inclusion criteria to determine any factors that did not appear in the primary studies. Critical appraisal of the identified relevant literature resulted in the identification of fifteen main factors that were deemed to influence EHRS implementation (see Table 2.3.1). Once all factors had been determined, I performed another scan of all relevant PDFs for further identification of facilitators and barriers. 
Table 2.3.1: Number of appearances of each included factor.

\begin{tabular}{|l|l|l|}
\hline No & Factors & $\begin{array}{l}\text { Number } \\
\text { publications }\end{array}$ \\
\hline $\mathbf{1}$ & Systems interoperability & appearances \\
\hline $\mathbf{2}$ & EHRS end-user satisfaction & 25 \\
\hline $\mathbf{3}$ & Project planning & 23 \\
\hline $\mathbf{4}$ & End-user involvement & 21 \\
\hline $\mathbf{5}$ & Training and support & 20 \\
\hline $\mathbf{6}$ & Project team selection & 20 \\
\hline $\mathbf{7}$ & Infrastructure & 17 \\
\hline $\mathbf{8}$ & Readiness & 17 \\
\hline $\mathbf{9}$ & Implementation cost & 15 \\
\hline $\mathbf{1 0}$ & Leadership and management & 14 \\
\hline $\mathbf{1 1}$ & EHRS efficiency and perceived & 13 \\
\hline & usefulness & 13 \\
\hline $\mathbf{1 2}$ & Usability & 11 \\
\hline $\mathbf{1 3}$ & Ethical and legal issues & 9 \\
\hline $\mathbf{1 4}$ & Software selection & 8 \\
\hline $\mathbf{1 5}$ & Communication among project team & 7 \\
\hline & & \\
\hline
\end{tabular}

Once all codes had been determined, I started to group all the initial codes into new codes using a hierarchical tree structure by looking for similarities and differences between the codes. Five themes were generated as a result of the hierarchical tree structure. Then, descriptive themes were reviewed and reduced to three themes by combining similar descriptive themes together to avoid any overlap and intervention. However, some of the determined factors still fitted under two or more themes. For instance, user involvement directly influences user satisfaction, but it still can be classified under organisational factors. 


\subsection{Results of the literature}

The term EHRS is sometimes referred to in different terms or applications (see Chapter One). However, for the purposes of this thesis, I used the term EHRS which includes other terms such as EMR, CDSS or CPOE. All the research included in this literature review ( $\mathrm{n}=76$ ) (see Table 11.8.2 in Appendix B) came from different countries (developed and developing) and were also conducted in both primary and secondary care. The most common place of publication was the USA $(n=32 ; 41.5 \%)$, followed by the UK $(n=9$; $11.7 \%)$.

According to the included papers, most of the factors that influenced EHRS implementation were identified in the period between 2000 and 2010. A variety of research methods have been used to explore EHRS implementation. Conducting systematic reviews to collate all factors that influence EHRS implementation have become common and widely used since 2010. The utilisation of qualitative and quantitative approaches to explore the implementation of EHRS were documented as the most frequently used methods since 2000. However, mixed method approaches for this type of research appear for the first time in 2009. Apart from systematic review research, four different data collection methods were detected. The most frequent data collection methods utilised were survey $(n=20,26.9 \%)$, followed by semi-structured interviews, which was reported in eighteen articles (23.8\%). Documentation analysis has been used only five times, and only two studies conducted observations. This indicates that semistructured interviews and surveys were the most popular and frequent data collection methods used to explore EHRS implementation.

EHRS implementation was explored in both secondary and primary care since 2000. According to the identified literature, all factors that influenced EHRS implementation were applicable to both secondary and primary care. End-user involvement, EHRS enduser satisfaction, project planning, implementation cost, training and support and EHRS interoperability were the most frequent factors influencing EHRS implementation (see Table 2.3.1). The most relevant papers to the current research that have been frequently used were published between 2006 and 2016 (see Table 2.4.1). 
Table 2.4.1: Key and most frequently used papers

\begin{tabular}{|c|c|l|}
\hline No. & Number of appearances & Reference \\
\hline $\mathbf{1}$ & 21 & Keshavjee et al., 2006 \\
\hline $\mathbf{2}$ & 20 & Ross et al., 2016 \\
\hline $\mathbf{3}$ & 18 & Gagnon et al., 2012 \\
\hline $\mathbf{4}$ & 18 & Ludwick and Doucette, 2009 \\
\hline $\mathbf{5}$ & 17 & Ghazisaeidi et al., 2014 \\
\hline $\mathbf{6}$ & 15 & Yusof et al., 2008 \\
\hline $\mathbf{7}$ & 12 & Lorenzi et al., 2009 \\
\hline $\mathbf{8}$ & 10 & Khajouei et al., 2011 \\
\hline $\mathbf{9}$ & 9 & Hoerbst and Schweitzer, 2015 \\
\hline $\mathbf{1 0}$ & 8 & Cresswell et al., 2013 \\
\hline
\end{tabular}




\subsection{Factors related to the organisation}

In the context of EHRS implementation, organisation refers to a healthcare organisation such as a hospital or PHC. In large-scale projects, the term organisation may refer to larger units such as the $\mathrm{MoH}$.

A clear relationship has been found between the size of healthcare organisations and the success of EHRS implementation (Ash \& Bates, 2005; Kruse, Kothman, et al., 2016). The successful adoption of EHRS has been documented as being worse in small practices compared with large ones (Ancker et al., 2013; DesRoches et al., 2012), with larger organisational size being documented as a facilitator to successful EHRS implementation (Jones \& Furukawa, 2014). However, others argued that smaller practices (i.e. PHCs) are less complex and less costly (Lennon et al., 2017).

The organisation level factors identified by this literature review can be considered under a number of main and sub-factors. The main factors identified were: project team selection, project team communication, planning, project leadership and management, readiness for change, workflow analysis and redesign, software selection and design, cost of the EHRS implementation project, training and support, and legal issues (see Table 2.5.1). 
Table 2.5.1: Factors influencing EHRS implementation at organisational level

\begin{tabular}{|c|c|c|}
\hline Factor type & Factors & \\
\hline \multirow{9}{*}{ Organisational factors } & Project team selection & $\begin{array}{l}\text { The involvement of } \\
\text { champions }\end{array}$ \\
\hline & $\begin{array}{c}\text { Project team } \\
\text { communication }\end{array}$ & \\
\hline & Planning & \\
\hline & Readiness to change & $\begin{array}{c}\text { Workflow analysis and } \\
\text { redesign }\end{array}$ \\
\hline & $\begin{array}{c}\text { Workflow analysis and } \\
\text { redesign }\end{array}$ & \\
\hline & $\begin{array}{c}\text { Software selection and } \\
\text { design }\end{array}$ & \\
\hline & $\begin{array}{l}\text { Cost of the EHRS } \\
\text { implementation project }\end{array}$ & \\
\hline & Training and support & The role of the super-user \\
\hline & $\begin{array}{c}\text { Privacy, confidentiality } \\
\text { and data security }\end{array}$ & \\
\hline
\end{tabular}

\subsubsection{Project team selection}

The selection of the project team is a vital contributing factor towards successful EHRS implementation (Adler, 2007; Carayon et al., 2009; Lorenzi et al., 2009). While some research has suggested that the formulation of an EHRS implementation project team should be the first step in the process of implementation (Adler, 2005; Sanchez et al., 2005), others have argued that the decision regarding composition of the project team is a step which should be taken only once the EHRS implementation plan has been approved (Ghazisaeidi et al., 2014). The project team are responsible for formulating a project plan, therefore the project team should be established early to ensure that all team members are involved in the early stage processes such as planning and decision-making. 
Moreover, for EHRS implementation to be successful, careful consideration of the qualifications of project team members has been highly recommended (Alkraiji et al., 2013). According to previous research, the selection of the project team should ensure a range of skills and take into consideration differences in backgrounds, disciplines and occupations (Hartzler et al., 2013). For instance, the project team should consist of an experienced project manager; a representative of the EHRS end-users; champions; project leaders; and technical staff responsible for implementing and supporting EHRS users (Simon et al., 2013). The responsibilities and tasks given to the project team should then be commensurate with their abilities and skills (Keshavjee et al., 2006; Sanchez et al., 2005).

\section{The involvement of champions in the implementation process}

The project champion role is important to the successful implementation of an EHRS (Ludwick \& Doucette, 2009b; Terry et al., 2008) and should be appointed in the early stages of implementation (Ghazisaeidi et al., 2014; Ross et al., 2016), especially the planning phase. The project champion's role is to act as a communication channel between project managers, project team members, and EHRS end-users (Yoon-Flannery et al., 2008), as well as the vendors of the technologies (Adams and Culp, 2005, Miller et al., 2003). The project champion also plays a role in enhancing staff acceptance levels (Lorenzi et al., 2009; Ovretveit et al., 2007).

\subsubsection{Communication among stakeholders}

Once the EHRS implementation project team has been selected and formed, an effective mechanism of communication between the selected project team members should be established (Fullerton et al., 2006; McAlearney et al., 2013; Yoon-Flannery et al., 2008). This requires coordination, cooperation, teamwork, and trust within the group (Lorenzi et al., 2009), as well as good communication with vendors (Keshavjee et al., 2006; Ludwick \& Doucette, 2009b). Effective project team communication should be established from the pre-implementation phase (Ghazisaeidi et al., 2014; Lorenzi et al., 2009). Effective communication between the project team and end-users can help to ensure wide end-user acceptance of the EHRS (Keshavjee et al., 2006; Lorenzi et al., 2009), and avoid any 
implementation issues such as systems interoperability or lack of training (Keshavjee et al., 2006; Ludwick \& Doucette, 2009b).

Potential misunderstanding of views and goals during the implementation process can be avoided by agreeing upon certain terms of communication (establishing robust contracts for instance), through co-ordination of distinct goals and objectives, by establishing a two-way communication passage between all parties (Keshavjee et al., 2006), and proper, on-going communication mechanisms to assist in the design of an adequate implementation plan (Yusof et al., 2008).

\subsubsection{EHRS end-user involvement}

A clear relationship has been identified between EHRS end-user involvement and their level of satisfaction (Gagnon et al., 2012; Hartzler et al., 2013; Hoerbst \& Schweitzer, 2015). There is a general consensus that involving EHRS end users in the implementation process, in particular while making important decisions such as software design and selection, is beneficial to EHRS implementation (Cresswell et al., 2013; Gagnon et al., 2012; Hartzler et al., 2013; Lorenzi et al., 2009; Scott et al., 2005). Fullerton et al. (2006); Gagnon et al. (2012); Keshavjee et al. (2006); Ovretveit et al. (2007); and Simon et al. (2013) have all illustrated that the involvement of EHRS end-users from various departments and occupations is essential to successful implementation. Therefore, implementation can be improved through consideration of the needs and requirements of every end-user (Cucciniello et al., 2015; Hartzler et al., 2013; McAlearney et al., 2013; Ross et al., 2016).

In the same context, in order to achieve smooth implementation, stakeholders need to be informed about the EHRS implementation process, including any future modifications to the system (Cucciniello et al., 2015; Gagnon et al., 2016). Moreover, to avoid any resistance, end-users should be treated as owners of the EHRS during the implementation (Boonstra et al., 2014; Gagnon et al., 2012). 
Previous literature has recommended defining specific time scales to involve EHRS endusers. While some researchers suggested involving EHRS end-users in the evaluation and readiness assessment (Cucciniello et al., 2015; Hartzler et al., 2013), others have suggested including them in the pre-implementation (e.g., planning) phase of the project (Ghazisaeidi et al., 2014). However, EHRS end-user involvement must be continuous and not confined to certain stages. A communication and involvement mechanism should exist throughout all phases of implementation (pre-implementation, implementation and post-implementation) (Cresswell \& Sheikh, 2013; Keshavjee et al., 2006; Lorenzi et al., 2009). Finally, the appointing of champions can assist in promoting the involvement of all EHRS end-users and other stakeholders (Hartzler et al., 2013).

\subsubsection{Project planning}

It is important to start planning the implementation of the EHRS in the early stages (Fullerton et al., 2006; Ghazisaeidi et al., 2014; Poon et al., 2004). The literature has emphasised the essential role of planning in the success of EHRS implementation (Cresswell \& Sheikh, 2013; Yusof et al., 2008). In addition, further evidence has shown that the majority of issues that lead to failure of IT projects are those relevant to poor planning (Keshavjee et al., 2006).

It has been suggested that the planning phase should take from one half to two-thirds of the actual implementation time (Adler, 2005). However, the suggested planning duration may not be applicable for long-term projects (i.e. large-scale projects). Ten-year projects, such as the implementation of EHRS in PHCs in SA (see Chapter One), may not be willing to spend five years formulating such a plan. The initial planning should consider the overall timescale of implementation, as well as resource requirements (Keshavjee et al., 2006).

Moreover, a good EHRS implementation plan should identify the structures already in the existing system (Ahmadian et al., 2014). Planners should consider important factors such as stakeholders' views and concerns, project values and goals, and EHRS end-user requirements (Yusif et al., 2017), as well as training courses and the cost of the EHRS project (Ghazisaeidi et al., 2014; Keshavjee et al., 2006) (see Figure 2.5.1). For better preparation in the planning phase, all project team members should be involved to ensure 
that their views and opinions are considered (Hartzler et al., 2013; Yusif et al., 2017). Furthermore, proper planning should discuss vendors, software selection and installation (Cucciniello et al., 2015). These plans also need to be feasible and fixable (Walker et al., 2006).

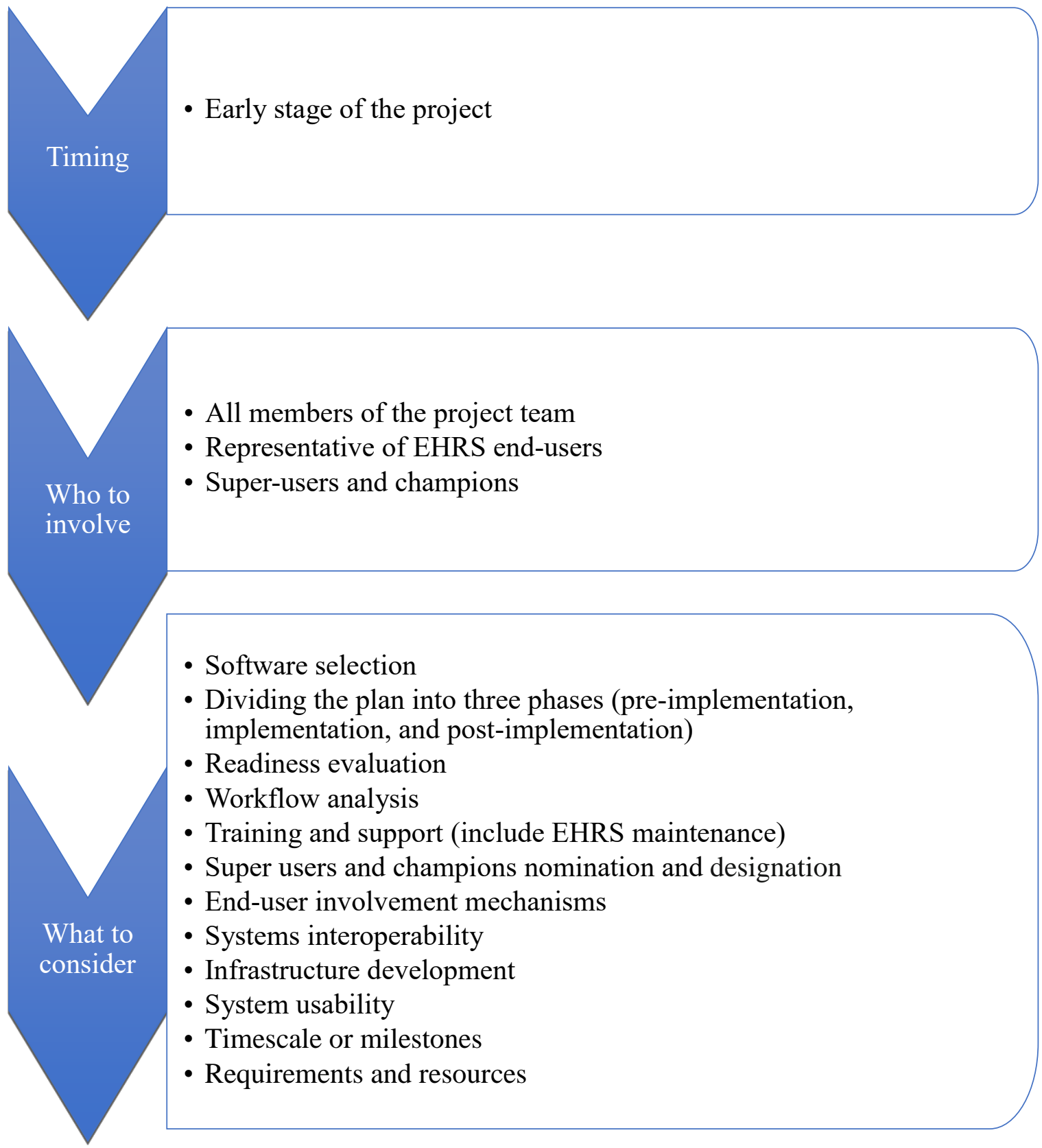

Figure 2.5.1: Description of good planning 
Effective planning can be divided into three different phases: "pre-implementation, implementation, and post-implementation" (Keshavjee et al., 2006). Phase division can help identify any barriers or facilitators to EHRS implementation in each phase and ensure smooth implementation (Keshavjee et al., 2006). Effective implementation must plan for probable risks (Adler, 2007). Risks can be detected through conducting situation analysis and readiness assessments (Ajami et al., 2011; Yusof \& Aziz, 2015).

Key elements of effective implementation planning have been highlighted by the included literature (see Figure 2.5.1). Firstly, a good plan must be established at the early stages of a project. According to the above literature, good planning should consider the main factors that influence EHRS implementation, such as software selection, readiness evaluation, workflow analysis training and support, super-user and champion roles, enduser involvement mechanisms, systems interoperability, commitment of adequate infrastructure, and system usability. Good planning requires inclusion of all the project team, as well as consideration of the requirements of end-users, and should be actioned across three different phases: pre-implementation, implementation, post-implementation. An effective plan will also consider the timescale for EHRS implementation. Finally, an appropriate plan is vital to ensure that healthcare organisations are at a good level of readiness for the EHRS implementation (Ross et al., 2016).

\subsubsection{Healthcare organisation readiness and workflow analysis}

As part of the planning procedure, it is important to consider undertaking a readiness evaluation of the healthcare organisation prior to implementation of a new EHRS. According to several studies, before EHRS implementation, an organisation has to be prepared to adapt to the change (Yusof \& Aziz, 2015; Yusof et al., 2008). Ross et al. (2016) have stated that readiness for EHRS implementation is an influential factor.

According to McAlearney et al. (2013), findings from readiness assessments are very useful tools that assist policy-makers in making better decisions and determining the barriers to implementation. For better and wider readiness assessments, factors from different dimensions, such as organisational, technological and human factors, should be considered (Ajami et al., 2011; Gagnon et al., 2016; Yusif et al., 2017). While some 
research has suggested conducting an assessment of healthcare organisation readiness during the early "pre-implementation phase" stages of the project (Ghazisaeidi et al., 2014; Ross et al., 2016), others have recommended conducting a readiness assessment initially and even before the planning phase (see Figure 2.5.2) (Ajami et al., 2011).

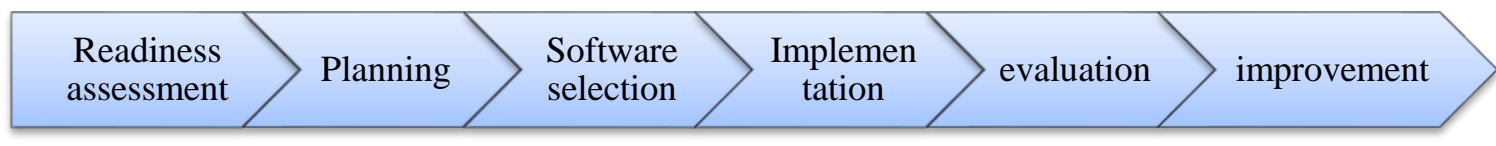

\section{Figure 2.5.2: EHRS implementation road map}

The vital aspects of readiness to implementation of EHRS have been identified as: individual factors; leadership; management; organisational culture; technical readiness; operational readiness: change management; training; and technical support (see Figure 2.5.3) (Ajami et al., 2011; Gagnon, Attieh, et al., 2014; Yusif et al., 2017).

The areas mentioned below should be mapped in order to evaluate the organisation's level of readiness:

- current IT systems and infrastructure

- experience in technology

- goals, motivations, requirements and attitudes

- potential constraints and issues

- economic and manpower resources (Keshavjee et al., 2006).

Work in the above-mentioned areas will aid in identifying specific aspects of an organisation that need attention and addressing before a new system (e.g., EHRS) can be implemented (Keshavjee et al., 2006). 


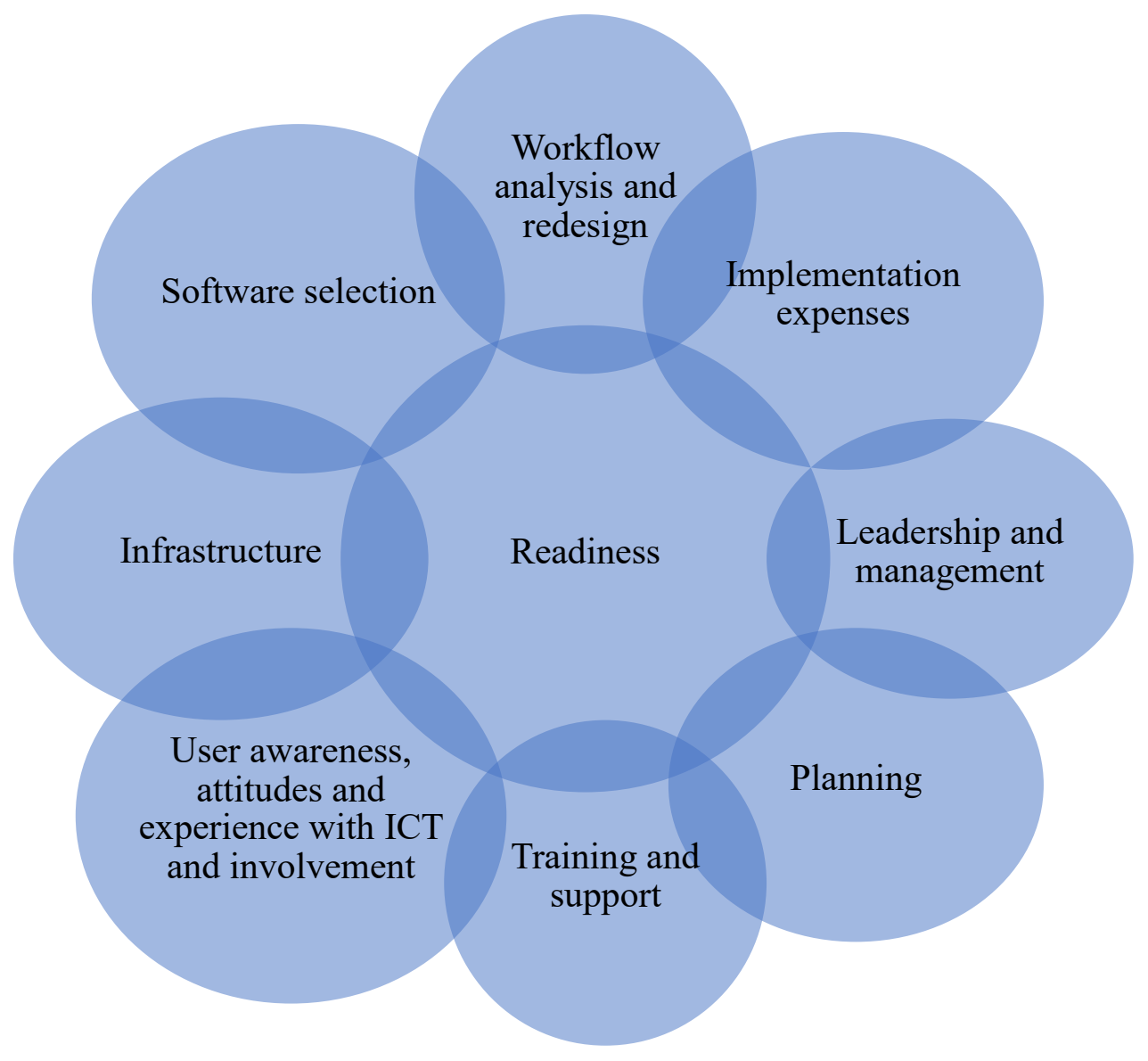

Figure 2.5.3: Factors to be considered during readiness assessments.

Readiness evaluation allows organisations to customise their systems during software selection, as well as providing adequate planning time to avoid any workflow disruption during the EHRS implementation (Fullerton et al., 2006). Therefore, organisational readiness for change is reported to be a crucial precursor to ensuring smooth and successful implementation of complicated changes in healthcare organisations (Ross et al., 2016). The main issues contributing to lower EHRS implementation rates are not just challenges arising from the process, but also the scarcity of pre-implementation resources and a low level of readiness for change (Biruk et al., 2014; Yusof \& Aziz, 2015).

Based on the identified literature, it seems sensible to conduct a readiness evaluation before the planning phase. The project team selection and formulation of a process should also be carried out before the planning phase. Hence, it is the project team's responsibility to conduct a readiness evaluation. Consequently, the collected data from the readiness assessment will assist with decision-making during the planning phase. For instance, a 
readiness evaluation may help with the selection of appropriate software, as well as the design of adequate training programs.

\section{Workflow analysis and redesign}

Workflow analysis and redesign are additional factors to be considered in a planning and organisational readiness assessment (see Figure 2.5.3) (Ghazisaeidi et al., 2014; Keshavjee et al., 2006; Lorenzi et al., 2009). The introduction of EHRS leads to changes in the organisation; these changes influence workflow and job functions. Therefore, conducting a workflow redesign will make EHRS implementation more effective (Ancker et al., 2013; Ghazisaeidi et al., 2014), assist in overcoming all organisational barriers and challenges (Ross et al., 2010), and can help reduce expenses and overcome financial barriers (Ross et al., 2016). However, workflow issues may hinder EHRS implementation (Ross et al., 2010).

\subsubsection{Cost of the EHRS implementation project}

Cost factors have a direct relationship with an organisation's readiness to introduce EHRS (see Section 2.5.5). Hence, the cost of EHRS implementation needs to be considered at an early stage (pre-implementation phase) (Ghazisaeidi et al., 2014), as part of a readiness assessment (Yusof \& Aziz, 2015). According to the studies of Cresswell et al. (2013); Kemper et al. (2006); and Whitacre and Williams (2015), such initial costs can be a hindrance to organisations considering an EHRS. Previous research has identified two different types of cost related to EHRS implementation: start-up cost (initial cost), such as that associated with the purchase of a new system (Slight et al., 2014; Walji et al., 2009); and ongoing costs, such as maintenance, training, developing infrastructure and provision of technical support (Cresswell et al., 2013; Ludwick \& Doucette, 2009a).

Low financial resources can become a barrier to successful implementation (Gagnon, Nsangou, et al., 2014; Kruse, Kristof, et al., 2016; Ludwick \& Doucette, 2009a). Kruse, Kristof, et al. (2016); Simon et al. (2007), identified cost as a major barrier to EHRS implementation, whereas Fritz et al. (2015), in their systematic review, argued that financial resources were found to be a minor factor influencing EHRS implementation. However, this argument appears in one study only, and, surprisingly, this study was 
conducted in a low-income country which one would expect to be more strongly influenced by finances than higher income countries. Finally, as mentioned earlier in this chapter, practice size was found to be an influencing factor, whereas larger healthcare organisations (i.e. hospitals) may face fewer financial difficulties than small healthcare organisations (i.e. PHCs).

\subsubsection{Software selection and design}

Software selection has been found to be another factor that needs to be considered early in the planning phase of EHRS implementation. The pre-implementation phase (including planning and readiness assessments) should identify the software requirements (Ghazisaeidi et al., 2014; Keshavjee et al., 2006). Therefore, enough time should be factored in to ensure that an appropriate system is identified, as there are many systems on the market of different sizes with different capabilities and features (Sanchez et al., 2005). As indicated by Boonstra et al. (2014); and Sanchez et al. (2005), executives and policy-makers must be involved in the selection of software. In addition, EHRS end user opinions must be considered and also involved in the software selection and design process (Cresswell et al., 2013; Hartzler et al., 2013) so as to enhance end-user satisfaction, ownership and confidence (Ross et al., 2016). It is also important to engage the champions and super-users when considering a new EHRS (Hartzler et al., 2013).

The price of the selected system, system interoperability and system usability are the most important criteria to consider when selecting an EHRS (Keshavjee et al., 2006; Khajouei et al., 2011). Hartzler et al. (2013) suggested that the selected system should be secure enough to protect patient data from any breach. On the other hand, software selection has been recorded as a barrier to EHRS implementation in PHCs, due to inadequacy of the current EHRS specifications for PHC requirements (Kemper et al., 2006). The majority of available EHRS have been designed for implementation in hospitals and larger organisations. 


\subsubsection{Ethical and legal issues}

As mentioned in the previous section, issues such as system security should be taken into consideration when the decision is being made to purchase a new EHRS. According to Fritz et al. (2015); Luna et al. (2014), "privacy, confidentiality and security" are the core ethical and legal issues that arise during the implementation and utilisation of EHRS in a healthcare organisation. This concern is due to data related to patients being stored and exchanged in the EHRS (Keshavjee et al., 2006). Concern about security and privacy of patient data is deemed a barrier that threatens EHRS implementation (Ajami \& ArabChadegani, 2013; Boonstra \& Broekhuis, 2010).

Privacy, confidentiality and security issues threaten the implementation of EHRS in developing and developed countries alike. For instance, EHRS implementation in developing countries (e.g., Saudi Arabia) has been negatively impacted by legal issues and is considered a challenge to both developers and policy makers in those countries (Luna et al., 2014). In addition, legal issues have been found to be a barrier to EHRS implementation in developed countries such as the USA, Canada, Norway and Ireland (Boonstra \& Broekhuis, 2010).

Privacy, confidentiality and security can be assured by organisations through the utilisation of number of policies. For instance, organisations can utilise audit logs, perform continuous monitoring and provide limited access permissions (privileges) to all EHRS end-users to avoid any issues related to privacy and confidentiality (FernandezAleman et al., 2013; Rezaeibagha et al., 2015). Moreover, further authentication and authorisation can help to protect patient privacy. For instance, all EHRS end-users need to be asked for a digital signature, electronic credentials, as well as smart cards (Fernandez-Aleman et al., 2013). To overcome the legal and ethical issues, Rezaeibagha et al. (2015); Ross et al. (2016) strongly suggested utilising technical standards to share and exchange patient information more securely and to ensure patient privacy and confidentiality are maintained. 


\subsubsection{Project leadership and management}

Project leadership and management have been found to be a crucial component of effective EHRS implementation (Cresswell et al., 2013; Fullerton et al., 2006; Walker et al., 2006; Yoon-Flannery et al., 2008). Project leadership and project management have been shown to have a positive impact upon EHRS implementation and increase the possibility of successful implementation (Lorenzi et al., 2009; Ludwick \& Doucette, 2009b; Rizer et al., 2015).

The project manager position can be occupied by either a technical staff member, physician, or any manager within the organisation (Walker et al., 2006). The project manager should have the required skills, receive training, be capable of leading (Ingebrigtsen et al., 2014), dealing with complex projects (Walker et al., 2006), and distributing workloads to project team members to encourage them to reach their goals (Sanchez et al., 2005). Moreover, integral to the lead project management role is enabling long-term communication between project team members (Ingebrigtsen et al., 2014) and ensuring the involvement of EHRS end-users (Rizer et al., 2015).

Smooth implementation requires expert leadership from an individual who has extensive experience of management hierarchy and with similar implementation projects (Ludwick \& Doucette, 2009b; Yusof et al., 2008). If higher-level management staff such as Chief Executive Officers (CEO), clinical managers or board members do not offer support, then EHRS implementation is more likely to fail (Ingebrigtsen et al., 2014). However, EHRS implementation may faces shortage in experts in this field (see Chapter One).

Effective leadership can be achieved by providing training, designating champions and super-users, follow-up, leadership support provision, decision-making and problemsolving, visioning, influencing, communication with the project team, team selection and strategizing (see Figure 2.5.4) (Ingebrigtsen et al., 2014). Leadership is essential when establishing an assessment of a healthcare organisation's readiness to implement EHRS (Ingebrigtsen et al., 2014). 


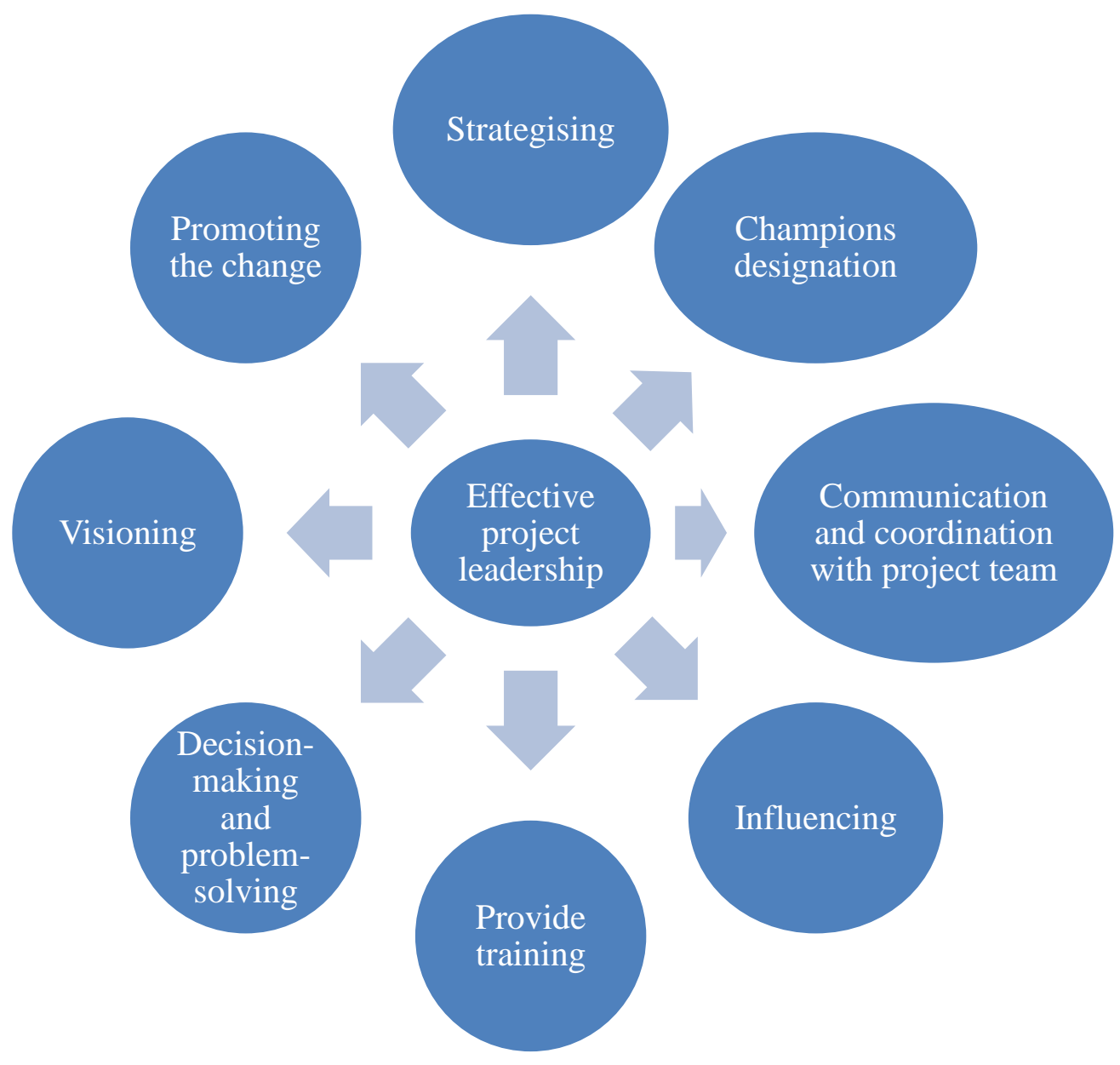

Figure 2.5.4: Components of a project leadership role in relation to EHRS implementation

Furthermore, the management need to be flexible and respond to all emerging issues relating to the implementation of the EHRS (Ingebrigtsen et al., 2014; Yusof et al., 2008). Despite the importance of the role of project management mentioned above, there are modest initiatives to highlight the role of centralisation in project management. Only one study stated that EHRS implementation can be improved through centralised project management (Safdari et al., 2015). Project leadership is essential when establishing an assessment of a healthcare organisation's readiness to implement EHRS (Safdari et al., 2015). 


\subsubsection{Training and support}

As illustrated in the previous section, one of the roles of leaders is to facilitate the provision of training and support. In this context, numerous articles have stated that training and support are influential factors in EHRS implementation (Adler, 2007; Ancker et al., 2013; Cresswell et al., 2013; Rizer et al., 2015; Ross et al., 2016; Safdari et al., 2015; Terry et al., 2009). While, Ross et al. (2016) have found that comprehensive and deliberate training and support are essential to the success of EHRS implementation, others have argued that a lack of technical support and inadequate training are barriers to EHRS implementation (Ancker et al., 2013; Terry et al., 2009).

\section{Training and support delivery timing and methods}

Initially, training in large-scale projects should be designed and delivered by a training team that comprises a training manager, training coordinator, instructional designers, a physician lead and super-users (Pantaleoni et al., 2015). It has been recommended that training and support should be provided in early phases (pre-implementation phase) of EHRS implementation (Fullerton et al., 2006; Ghazisaeidi et al., 2014; Ludwick \& Doucette, 2009b). However, others have suggested training sessions should commence no more than eight weeks prior the go-live phase (Pantaleoni et al., 2015), as training at such an early stage may be forgotten by the trainees. It has also been recommended that ongoing training and technical support should be provided to the EHRS end-user (Adler, 2007; Ash et al., 2012; Pantaleoni et al., 2015).

It has been recommended that the training phase should be preceded by an awareness campaign to introduce the new project (Alkraiji et al., 2013). The process of generating awareness about the change, before it happens, should present an explanation of the reasons behind the change, the necessity for a new system, and the way the change will be managed. It should also encourage feedback (Walker et al., 2006). In addition, all EHRS end-users and other stakeholders should be aware of the new change (Fullerton et al., 2006). 


\section{The role of the super-user in providing training and support}

Members of a super-user team are usually organisation personnel who have already received education and training to guide the change process, provide instruction, communicate and continuously support other stakeholders (Keshavjee et al., 2006; Pantaleoni et al., 2015; Simon et al., 2013). Any healthcare practitioner can be a superuser, including physicians, nurses, or pharmacists (Simon et al., 2013). The role of the super-user is to provide extra training to users and ongoing, one to one support (Simon et al., 2013).

Similar to the role of the champion, super-users act as a communication channel between the project team and other stakeholders, which can help to improve the level of acceptance among EHRS end-users (Gagnon et al., 2012; Yoon-Flannery et al., 2008). Moreover, the role of the super-user is more prominent in large-scale projects to implement an EHRS in secondary care (Simon et al., 2013). Overall, the identification of super-users has had a positive impact on EHRS implementation (Boonstra et al., 2014; Simon et al., 2013; Yusof \& Aziz, 2015). Training and support were the last organisational factors to influence EHRS implementation. The following section will address the technological factors that influence EHRS implementation. 


\subsection{Technological Factors}

Technology here refers to the EHRS, thus all technological factors are those related to the EHRS itself (e.g., EHRS usability and EHRS efficiency), or other factors that have a direct relationship with the EHRS (e.g., infrastructure and interoperability) (see Table 2.6.1).

Table 2.6.1: Factors influencing EHRS implementation at a technological level

\begin{tabular}{|c|c|}
\hline Factor type & Factors \\
\hline \multirow{2}{*}{ Technological factors } & Systems interoperability \\
\cline { 2 - 2 } & System usability \\
\hline & $\begin{array}{c}\text { System efficiency and } \\
\text { perceived usefulness }\end{array}$ \\
\hline
\end{tabular}

Technological factors have been found to have an impact on the success or failure of EHRS implementation (Gagnon et al., 2012; Kruse, Kothman, et al., 2016; Ross et al., 2016). As mentioned earlier in this chapter, technological readiness to introduce a new EHRS has been documented as a core element of project implementation and an essential factor that can predict the barriers to successful implementation in the early stages. For instance, according to Ajami et al. (2011), readiness assessments are a useful method for developing good infrastructure.

Factors influencing the implementation of EHRS at a technological level have been identified as: systems interoperability (Cresswell et al., 2013; Hoerbst \& Schweitzer, 2015; Kruse, Kothman, et al., 2016), system usability (Cresswell et al., 2013; Hoerbst \& Schweitzer, 2015; Ludwick \& Doucette, 2009b), infrastructure (Bates et al., 2003; Fritz et al., 2015), and system efficiency (Bates et al., 2003; Cresswell et al., 2013; Gagnon et al., 2012). 


\subsubsection{System efficiency and perceived usefulness}

A direct association between system efficiency and EHRS implementation success has been identified (Khalifa \& Alswailem, 2015; Kruse, Kothman, et al., 2016; Kruse et al., 2015; Yusof et al., 2008). Six efficiency related factors should be considered to improve EHRS implementation: accessibility, stability, flexibility, reliability, adaptability, and EHRS performance. Cresswell et al. (2013); Gagnon et al. (2012); Gagnon et al. (2016); Hoerbst and Schweitzer (2015) argued that system efficiency (e.g., performance, response time and accessibility) considerations should be looked at in the early stages of software selection or design.

Previous literature has indicated that using the new EHRS should not take longer than the existing traditional system (paper-based system). An inefficient or slow EHRS has often been considered an obstacle to EHRS implementation (Pare et al., 2008; Yusof et al., 2008). However, system performance can be improved, over time, to meet healthcare practitioner's expectations by taking into consideration end-user input and feedback and performing regular evaluations (Ross et al., 2016). Yusof et al. (2008) revealed that system quality is an influential factor in EHRS end-user satisfaction.

Perceived usefulness has also been recorded as an influential factor in EHRS implementation (Gagnon et al., 2012; Kruse, Kothman, et al., 2016). EHRS implementation can be more likely to succeed if it is characterised by an unambiguous comprehension of the advantages of the new system among users (Gagnon et al., 2012). Numerous studies have detected a direct association between user satisfaction and perceived usefulness of EHRS (Bani-Issa et al., 2016; Gagnon et al., 2012; Hoerbst \& Schweitzer, 2015; Kruse, Kothman, et al., 2016). Lack of perceived usefulness can increase the level of resistance to a system in end-users (Kruse, Kristof, et al., 2016).

\subsubsection{System usability}

System usability has been identified as having a major influence on EHRS implementation (Cresswell et al., 2013; Khajouei et al., 2011). Usability issues can act as constraints to the implementation of IT in healthcare organisations (Gagnon, Nsangou, et al., 2014; Khajouei et al., 2011; Kruse, Kristof, et al., 2016; Middleton et al., 2013; Yusof 
et al., 2008). On the other hand, positive relationships between system usability and adoption rate have been documented, a high level of system usability increases the adoption rate (Ludwick \& Doucette, 2009b). Moreover, EHRS usability is one of the main factors associated with end-user satisfaction with the system (Gagnon et al., 2016; Khajouei et al., 2011).

The usability of software can be enhanced through adequate system design (Keshavjee $e t$ al., 2006; Ludwick \& Doucette, 2009b). Therefore, previous research has suggested that to avoid any usability issues, EHRS designers should consider several criteria: humantechnical interaction, ability to produce feedback, system learnability, system flexibility and customisability, visibility, graphic and interface design, clear information presentation, minimisation of information overload and limited number of screens and orders, avoidance of errors, software navigation and modification, consistency, memorability, and use of proper language (Middleton et al., 2013; Zahabi et al., 2015). However, usability issues are not detectable prior to the actual implementation of the EHRS (Khajouei et al., 2011). Thus, the project team should consider an early pilot implementation phase to detect any issues that may lead to implementation failure (Fullerton et al., 2006; Walji et al., 2009).

Usability evaluation studies should take into consideration system efficiency, end-user satisfaction and system effectiveness (Khajouei et al., 2011). Numerous studies have documented adverse effects on the quality of care, medication errors, EHRS end-user errors, and patient safety resulting from usability issues (Khajouei et al., 2011; Middleton et al., 2013; Zahabi et al., 2015).

\subsubsection{Systems Interoperability}

EHRSs interoperability has been highlighted as an influential factor in their implementation (Gagnon, Nsangou, et al., 2014; Gagnon et al., 2016; Kruse, Kothman, et al., 2016; Lennon et al., 2017; Ludwick \& Doucette, 2009b; Ross et al., 2016; Terry et $a l ., 2008)$. The basis for systems interoperability lies in a common understanding of the data codes and concepts among EHRS (Arvanitis, 2014). Unsuccessful interoperability may occur due to misconnection or discoordination between providers of different 
healthcare organisations, as different organisations utilise different types of standards (Luna et al., 2014). Arvanitis (2014) divided systems interoperability in healthcare into two dimensions, titled "syntactic interoperability" and "semantic interoperability". Semantic interoperability is a core factor in large-scale projects (Hoerbst \& Schweitzer, 2015).

Decisions regarding systems interoperability need to be planned at an early stage and prior to the selection and purchase of software (Alkraiji et al., 2013; Keshavjee et al., 2006). Thus, to ensure smooth interoperability, EHRSs need to be tested and evaluated in the pre-implementation phase (Keshavjee et al., 2006). Previous literature has identified a clear relationship between the level of usability and EHRS interoperability (Middleton et al., 2013). Therefore, commercial EHRS are preferable for policy makers, in largescale implementation projects, due to their flexibility and their ability to integrate with other systems (Cresswell et al., 2013).

Previous literature has illustrated that non-interoperable systems may increase expense. For instance, interoperability issues in the UK posed a significant challenge to the implementation of a national EHRS (Luna et al., 2014). Although the US government spent approximately $\$ 30$ billion to implement an interoperable EHRS (Rao et al., 2011), only $16 \%$ of US hospitals have interoperable systems (Kruse et al., 2014). Adequate interoperability can facilitate cost reduction (Jardim, 2013), reduce medication error (Kruse et al., 2015), improve access and patient data retrieval (Rezaeibagha et al., 2015), and improve the quality of healthcare provided to patients (Jardim, 2013). In addition, interoperability can encourage small healthcare organisations, such as GPs, to adopt the EHRS (Jardim, 2013). The importance of interoperability for PHCs can be summarised into three functions: sending e-referrals, sending medical messages to other healthcare providers (e.g., hospitals), and producing reports and summaries to transfer patients to secondary care (Gordon et al., 2015). From the above statements, it can be concluded that systems interoperability is the backbone to the success of larger scale projects, such as national EHRS.

Developing countries seem to be lagging behind developed countries. For example, poor interoperability due to differences between the implemented systems in different hospitals has been detected in SA (Aldosari, 2014). Internationally, an effort is in progress to unify 
a standard to enhance EHRS interoperability in developing countries (Luna et al., 2014). To overcome interoperability issues, EHRS implementation should be fully compliant with such a standard (e.g., HL7) (Alkraiji et al., 2013).

\subsubsection{Technical infrastructure}

Infrastructure has been documented as an influential factor in EHRS implementation at the technological level (Alkraiji et al., 2013; Bates et al., 2003; Fritz et al., 2015; Ross et al., 2016; Ross et al., 2010). Two main elements of technical infrastructure have been defined: hardware (e.g., computers, tablets, printers, scanners, smart cards, cables, mobiles, landline, routers to access the internet and switches), and software, including data warehouses, project and change management software, learning software, licences and antivirus software (Ahmadian et al., 2014; Slight et al., 2014).

A negative relationship has been found between low resources and the infrastructure, with poor infrastructure often detected in low income countries (Biruk et al., 2014; Fritz et al., 2015). However, both developed (Bates et al., 2003; Wang \& Biedermann, 2012) and developing countries (Ahmadian et al., 2014; Luna et al., 2014) have been exposed to infrastructure issues. Preparing adequate infrastructure is very costly, when compared with other factors that influence EHRS implementation (Ross et al., 2010). The quality of infrastructure may vary from one country to another, or even one region to another within the same country (Luna et al., 2014). Infrastructure challenges can be overcome, when considered in the early and planning phases of a project (Cresswell et al., 2013).

Previous studies have noted that poor infrastructure often coincides with the geographical location of a healthcare organisation, with remote and rural areas experiencing more issues as a result of poor infrastructure (Singh et al., 2012; Whitacre \& Williams, 2015). Researchers have revealed several possible reasons for poor infrastructure in developing countries, including, for example, poor internet connectivity (Luna et al., 2014). 


\subsection{Factors related to the human (individual)}

This section provides a description of the factors associated with EHRS implementation at the human level. Human in this section refers to individuals who have a direct or indirect relationship to the implementation or use of an EHRS. These individuals include administrative staff (e.g., receptionists) or healthcare practitioners (e.g., physicians and nurses). Human factors can be considered under a number of main and sub-factors: (see Table 2.7.1). A strong association between EHRS end-user acceptance and attitudes and the success of EHRS implementation projects has been suggested (Cucciniello et al., 2015; Khajouei et al., 2011; Ludwick \& Doucette, 2009b; Yusof et al., 2008).

Table 2.7.1: Factors influencing EHRS implementation at human level

\begin{tabular}{|c|c|}
\hline Factor level & Factors \\
\hline \multirow{2}{*}{ Human factors } & Individual readiness \\
\cline { 2 - 2 } & User satisfaction \\
\hline
\end{tabular}

\subsubsection{Individual readiness for EHRS implementation}

As mentioned earlier in this chapter, organisational readiness for a new technology has been found to be an influential factor in EHRS implementation. This section will highlight the role of individual readiness to a new technology and other factors that correlate with the level of individual readiness. Ajami et al. (2011); Biruk et al. (2014); and Ludwick and Doucette (2009b) have suggested that individual factors are core elements that need to be considered when conducting a readiness assessment. This is due to the significant impact that individual readiness has on the success of EHRS implementation (Hoerbst \& Schweitzer, 2015). Biruk et al. (2014) have illustrated how some healthcare organisations did not attempt to consider human readiness, when conducting readiness assessments. Human readiness can be divided into two categories: end-user involvement readiness, and core readiness. Involvement readiness is described as "active willingness and participation of people for EHRS implementation", whilst core readiness is defined as "the realization of needs and expressed dissatisfaction with the current way of working" (Biruk et al., 2014 p.3). 
Individual readiness assessments can be conducted by including eight criteria: computer skills, gender, awareness about the perceived usefulness of the system, attitudes toward the implementation of the EHRS, knowledge about the EHRS implementation (Biruk et al., 2014; Terry et al., 2008; Yusif et al., 2017), experience at work, age (Yusif et al., 2017), and willingness (Lennon et al., 2017; Yusif et al., 2017).

\subsubsection{EHRS end-user satisfaction}

The overall level of end-user satisfaction toward EHRS use and implementation is still low (Lorenzi et al., 2009; Yusof et al., 2008). Therefore, EHRS end-user satisfaction is considered to be one of the key measurement criteria of end-user attitudes and perceptions toward EHRS implementation (Ancker et al., 2013; Holanda, do Carmo, et al., 2012; Lorenzi et al., 2009). According to Ross et al. (2016), the level of EHRS satisfaction may be influenced by several factors, such as concerns about losing productivity, privacy and security, losing autonomy, and fears regarding liability. Hence, EHRS end-users may believe that using such EHRS may influence their relationship and direct communication with the patients, and also affect their responsibilities.

Several studies have been undertaken to explore the factors that affect EHRS end-user satisfaction. The literature has identified a relationship between EHRS satisfaction and factors related to technology and organisation (see sections 2.5 and 2.6). For instance, system usability, perceived usefulness and system efficiency were recorded as technological factors that influence user satisfaction (Gagnon et al., 2012; Hoerbst \& Schweitzer, 2015; Kruse et al., 2015; Ross et al., 2016). Moreover, training, technical support and end-user involvement influence EHRS end-user satisfaction at the organisational level (Ash et al., 2012; Hoerbst \& Schweitzer, 2015; Keshavjee et al., 2006; Ludwick \& Doucette, 2009b; Ross et al., 2016). Direct association between EHRS end-user satisfaction and demographic characteristics was detected (such as age, gender, experience with the system, computer literacy, and occupation) (Ahmadian et al., 2014; Gagnon et al., 2012; Gagnon, Ghandour el, et al., 2014; Khajouei et al., 2011; Kruse, Kothman, et al., 2016; Yusif et al., 2017; Yusof et al., 2008). Therefore, lack of computer experience is considered to be a barrier to effective implementation of EHRS (Terry et al., 2009). Some, however, have documented that the relationship between EHRS end- 
user satisfaction and computer experience in SA is not well understood (Alasmary et al., 2014). In terms of experience with using EHRS, Khajouei et al. (2011) have indicated that EHRS end-user performance and satisfaction was enhanced with frequent use of the system. Hence, the level of satisfaction can increase over time (Holanda, do Carmo, et al., 2012).

\subsection{Summary}

The purpose of this review was to describe the relevant factors associated with EHRS implementation, identified by the literature. My aim with this literature review was to provide a better understanding of the obstacles that may hinder the success or failure of EHRS implementation, as well as provide a better understanding of the facilitators that may enhance its success. The literature revealed fifteen main factors, which can be categorised under three main themes: organisational, technological, and human factors. These themes were broadly similar to those identified by Yusof et al. (2008) in their HOTfit framework. Several additional factors were identified, however, such as EHRSs interoperability.

It has been suggested that any EHRS implementation should begin by selecting the most appropriate individuals. Thereafter, the literature suggested establishing a communication channel among the project team to formulate an adequate project plan. It is essential to conduct readiness assessments, prior to any EHRS implementation, to avoid any issues and to make better decisions concerning aspects such as budget allocation. Moreover, workflow analysis and redesign were also identified as factors that influence implementation. The cost of EHRS implementation was a challenge to the majority of previous projects, and also hindered some healthcare organisations. Software selection is therefore an influential factor. Criteria such as security, confidentiality and privacy need to be considered during software selection; criteria that were reported as a main concern of stakeholders when using an EHRS. Further technical criteria need to be considered during the software selection, these include EHRS usability, efficiency and interoperability. On the other hand, training and technical support are the most controversial and influential issues among organisational factors. Shortcomings in any of these areas may be a barrier to an EHRS implementation project. 
Technical infrastructure, usability, efficiency, interoperability and perceived usefulness of the EHRS have been classified as technological factors that can affect the success of EHRS implementation. At a human level, EHRS end-user satisfaction was recorded as a vital influencing factor in EHRS implementation. A relationship exists between EHRS end-user satisfaction and several organisational factors (i.e. training and support) and technological factors (i.e. usability and efficiency). Furthermore, EHRS end-user demographics, such as user age, gender and profession, can make a difference in terms of level of satisfaction. Although the majority of the determined factors can become a barrier to EHRS implementation, poor readiness, inadequate leadership and project management were documented as being the main cause of project failure.

\section{Gaps in research}

According to this literature review, there remain several gaps in the research on EHRS implementation. Firstly, the literature review revealed that no research has been conducted to explore or evaluate EHRS implementation in PHCs in SA, and only a handful studies have been conducted in developing countries. All the research to date that has been conducted in SA regarding EHRS implementation has taken place in secondary care environments, such as public, private and maternity hospitals. Moreover, although numerous studies have been carried out to examine the implementation of EHRS in PHCs internationally, these have been conducted on single or small group centres. No previous research has examined the implementation of large-scale EHRS in PHCs, in particular for those projects with centralised management.

Furthermore, reviewing previous literature detected several gaps about the influence of some factors on the EHRS implementation in the PHCs, especially large-scale projects. Firstly, the communication mechanism among project teams and stakeholder involvement in large scale projects are not well-defined in the literature, especially projects with CM. Therefore, this thesis will attempt to provide an in-depth description of the communication and stakeholder involvement mechanisms used during implementation. This has been carried out via semi-structured interviews with the project team (see Chapter Five). 
Although there has been much research investigating the negative impact of EHRS implementation costs, no previous study has evaluated whether the availability of sufficient FR has a positive impact or not. Furthermore, as mentioned in Chapter One, the Saudi MoH utilised CM to manage implementation in PHCs. However, only one study has mentioned the role of CM (Safdari et al., 2015). Therefore, I explored these two gaps by conducting a questionnaire-based study and semi-structured interviews (see Chapter Four and Seven).

Searching through the literature resulted in a lack of identified research that has measured healthcare organisation readiness for implementing large-scale EHRS projects, particularly in PHCs. Moreover, there is a shortage of research regarding healthcare organisation readiness for EHRS implementation in developing countries, and therefore may require greater readiness evaluation research, as developing countries are lagging behind developed countries in terms of preparedness for EHRS infrastructure (Luna et al., 2014). Consequently, this thesis assessed the readiness of PHCs for the implementation of a large-scale EHRS at different levels, including technological, organisational and individual levels, through the use of questionnaire-based research and semi-structured interviews (see Studies One, Two and Four).

At the individual level, no study has examined the impact of individual demographic differences in PHCs in SA, such as age, gender and profession. Therefore, this thesis evaluated the impact of these characteristics on satisfaction with the EHRS (see Chapter Six). In the same context, the main focus of previous studies was on nurses and physicians. The majority of previous studies failed to include other EHRS end-user parties, such as receptionists and lab technicians. Gagnon, Nsangou, et al. (2014), in their recent systematic review, argued that perception and satisfaction of EHRS end-users, other than physicians and nurses, has been neglected by previous literature. Moreover, some have documented that the relationship between EHRS end-user satisfaction and computer experience in SA is not well understood (Alasmary et al., 2014). Therefore, this thesis included other EHRS end-user groups, such as pharmacists, receptionists and lab technicians. 
At a technological level, no previous study has been conducted to evaluate the influence of efficiency, usefulness and usability on the implementation of an EHRS in PHCs in SA, hence these aspects have been examined in Chapter Six. Furthermore, the impact of the technical infrastructure is poorly understood in terms of implementing large-scale EHRS in PHCs. Therefore, these factors have been examined in Studies Three and Four.

Overall, this thesis attempted to fill the identified gaps in the literature through the utilisation of four studies, one qualitative and three quantitative. Two studies were directed to the project team to assess the readiness of PHCs for EHRS implementation, to describe the implementation of the EHRS, and to evaluate the impact of the FR and CM. The other two studies were conducted from PHC staff and end-user perspectives to evaluate the implemented EHRS and the PHCs readiness for EHRS implementation

The next chapter describes in detail the research design, methodology and methods, which were used in each of each of the studies, how these studies were conducted, how the data were analysed, and the findings. 
3 Chapter Three: Research Methodology 


\subsection{Introduction}

This thesis aims to explore the large-scale implementation of EHRS in PHCs in SA. As identified in the literature review (Chapter Two), EHRS implementation is influenced by several factors related to the individual, organisation and technology. These determined factors will be taken into consideration, during readiness assessment and during the evaluation of the implemented EHRS, as indicators of project success or failure. The factors identified by the literature review will be examined in the thesis, using the methods described in this chapter.

The literature review revealed a gap in regard to the impact of Centralised Management (CM) on EHRS implementation. It also revealed a gap with regard to impact of Financial Resources (FR) to facilitate EHRS implementation. In addition, no previous studies have been conducted to evaluate EHRS implementation in PHCs in SA or assess the readiness of these centres to introduce EHRS. Consequently, the barriers and facilitators to successful implementation of EHRS in PHCs in SA are not well understood.

This chapter describes the research design and methodological approach used to explore EHRS implementation in PHCs in SA, as well as the data collection methods that underpinned the current thesis, in more detail. To achieve the main aim of this thesis, I utilised a mixed-methods approach which included both qualitative and quantitative methods (Bryman, 2012; Creswell, 2014) (see Figure 3.1.1). Firstly, a questionnairebased study was used to assess PHC readiness for EHRS implementation. This was followed by two further questionnaire-based studies: one of these was conducted to assess PHC readiness for EHRS implementation from the perspective of PHC staff, and the second evaluate the implemented EHRS from the end-user perspective (see Figure 3.1.1). Finally, semi-structured interviews were carried out to explore the views of the project team regarding PHC readiness for EHRS implementation and then used to determine the barriers and facilitators to its implementation, as well as to evaluate the impact of FR and $\mathrm{CM}$ (see Figure 3.1.1). While qualitative semi-structured interviews provided an opportunity for in-depth exploration (Bryman, 2012), questionnaires allow widespread coverage, are relatively cheap, and are less time consuming (Bryman, 2012). 
To explore the large-scale implementation of EHRS in PHCs in SA

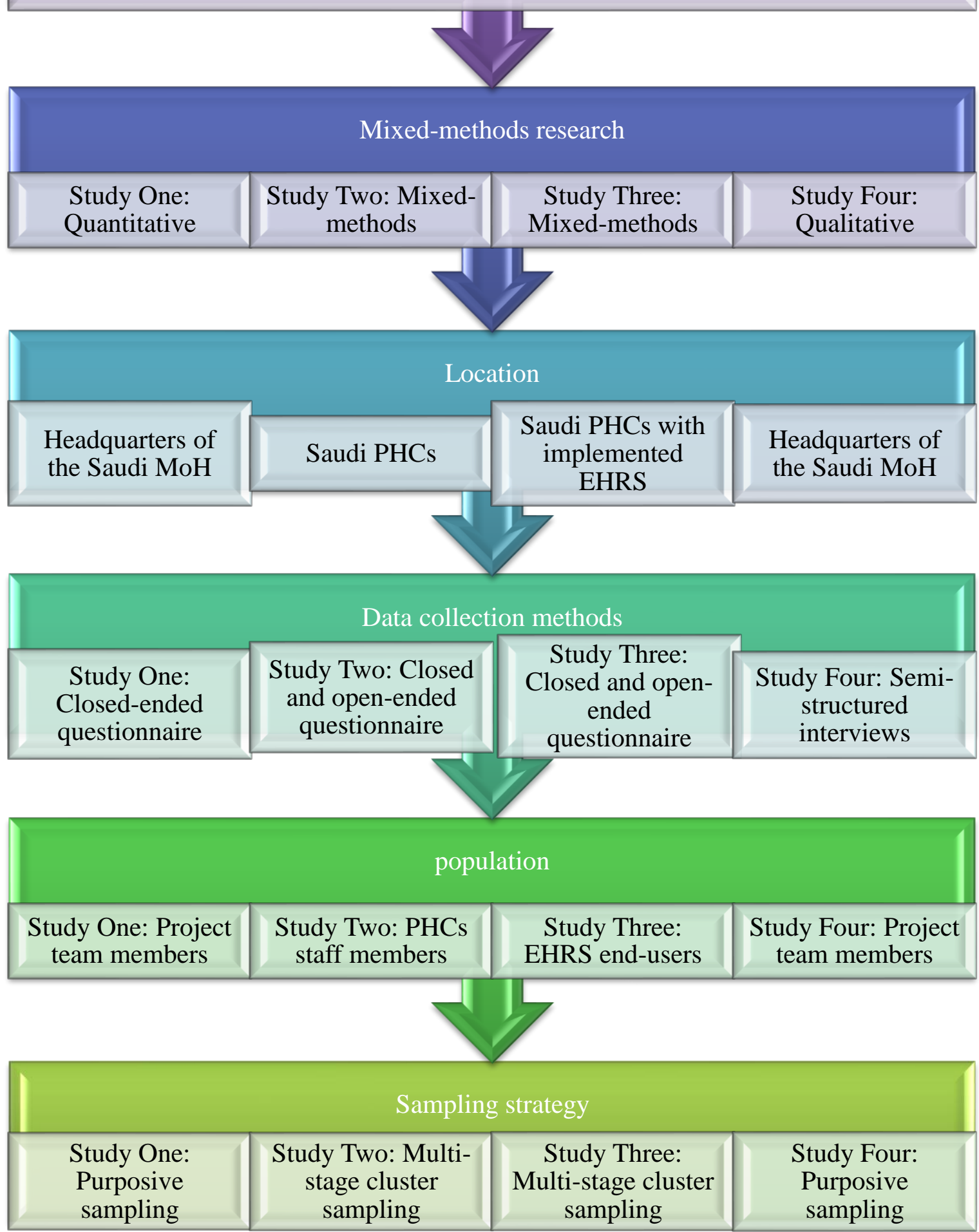

Figure 3.1.1: Methodology and methods employed in the thesis 
As mentioned in Chapter One, the Saudi $\mathrm{MoH}$ is planning to implement a large-scale EHRS in PHCs. The MoH had a previous unsuccessful attempt to implement an EHRS in PHCs. Thus, for better insight and wider exploration of the implementation of EHRS, the conducted studies aimed to explore both previous and current projects. As revealed in Chapter Two, EHRS implementation can be divided into three phases: preimplementation, implementation, and post-implementation (see Figure 3.1.2) (Keshavjee et al., 2006).

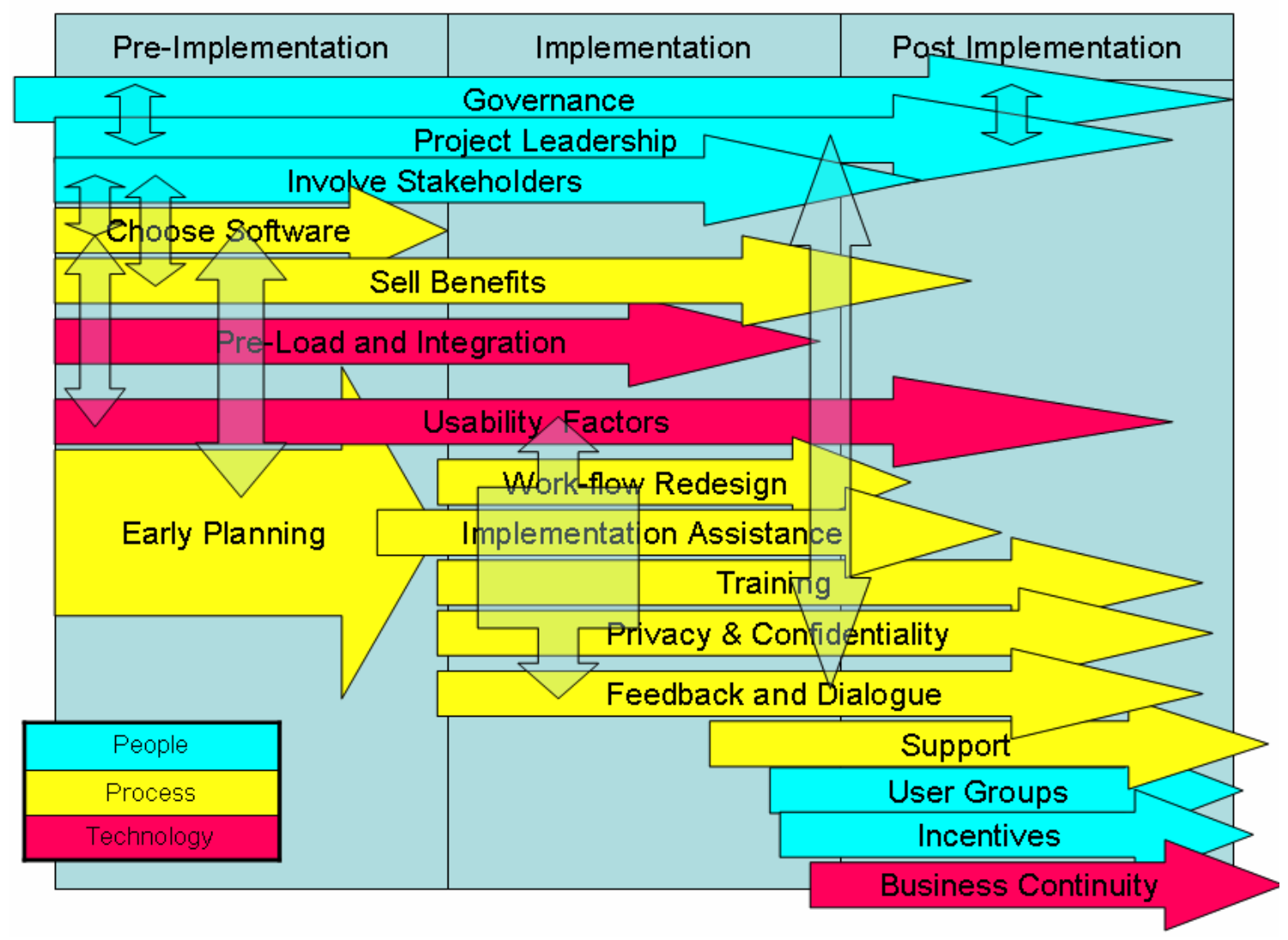

Figure 3.1.2: People, Process and Technology Model (PPTM)

Table 3.1.1 shows the coverage of each study, in this thesis, in accordance with the implementation phases. As seen in the below table, only pre-implementation and postimplementation were examined in this thesis. Therefore, this thesis explored those two phases only by assessing PHC readiness (pre-implementation) and evaluating the implemented EHRS (post-implementation). 
Table 3.1.1: Study coverage based on implementation phases

\begin{tabular}{|l|l|l|l|l|}
\hline & $\begin{array}{l}\text { Conducted } \\
\text { date }\end{array}$ & $\begin{array}{l}\text { Pre- } \\
\text { implementation } \\
\text { phase }\end{array}$ & $\begin{array}{l}\text { Implementation } \\
\text { phase }\end{array}$ & $\begin{array}{l}\text { Post- } \\
\text { implementation } \\
\text { phase }\end{array}$ \\
\hline $\begin{array}{l}\text { Study } \\
\text { One }\end{array}$ & $\begin{array}{l}\text { October, } \\
2014\end{array}$ & Yes & No & No \\
\hline $\begin{array}{l}\text { Study } \\
\text { Two }\end{array}$ & $\begin{array}{l}\text { January, } \\
2016\end{array}$ & Yes & No & No \\
\hline $\begin{array}{l}\text { Study } \\
\text { Three }\end{array}$ & $\begin{array}{l}\text { January, } \\
2016\end{array}$ & No & No & Yes \\
\hline $\begin{array}{l}\text { Study } \\
\text { Four }\end{array}$ & $\begin{array}{l}\text { October, } \\
2014\end{array}$ & Yes & No & Yes \\
\hline
\end{tabular}

\subsection{Ethical considerations}

Prior to any data collection, the first step was to obtain ethical approval to carry out the research. Therefore, two approvals were submitted and granted before starting the data collection. The first was obtained on $16^{\text {th }}$ January 2014 from the College of Human and Health Sciences (CHHS) and Swansea University Medical School (SUMS) Research Ethics Committee (REC) (see Appendix A). The second approval was gained from the External Research Review Committee (ERRC) at the Saudi MoH on $18^{\text {th }}$ of August 2014. The approvals covered all four studies conducted as part of the thesis. Both committees received a detailed description of the nature of the studies, the targeted population for each study, and steps taken by me to ensure that all obtained data were secure and anonymous.

As a result of these approvals, I was able to have direct contact with all the relevant departments and individuals in order to undertake the research. This included the heads of all the related departments, team members of EHRS implementation project, and directors of some PHCs (see Appendix A). Consent was gained from all participants.

The participant information sheets, for the questionnaire-based research, explained the reasons for the study and why the participant had been approached. Participants were told that they could withdraw at any time, even if they had agreed to participate initially. 
Furthermore, the participants were told that no benefits would be offered in return for their participation. The participants were informed that their participation in the study was valued and that it may help make the process of EHRS implementation within PHCs more efficient and effective. All the participants were told that the data gathered would be fairly processed and analysed. The privacy and anonymity of the participants was assured. I indicated to the participants that they would come to no mental or physical harm during the process of gathering data. In the final stage, my contact details were provided so that the participants could make contact if they had any concerns regarding the process and the subsequent treatment of the information gathered. In the case of questionnaire-based studies, returned questionnaires were considered as implied consent from the participant to use the data.

Apart from the above mentioned measures, Barbour (2013) suggests that it is vital for the interviewees and other participants to know about the consent: so I made sure that the participants understood the information provided and what the study involved, and could therefore provide informed consent. For example, it was important that the participant understood that the personal information they provided would be treated confidentially and would not be disclosed to other people.

The researcher also needed to consider the issue of ethical conflict that could occur in the future (Silverman, 2013). In the qualitative research study, I obtained consent from the participants by using an informed consent form (see Appendix A). This was completed prior to carrying out the research with each individual participant. The data collected from the qualitative interviews included audio recordings, interview notes, digital files, transcripts, information in the questionnaires and digital data in SPSS. These data were stored in a hard drive that was protected by a password. The data was archived following submission of the thesis in accordance with Health Research Authority guidance for research (see Appendix A). 


\subsection{Study One: Assessing PHCs readiness for the implementation of the EHRS from the project team perspective}

This study aimed to assess Saudi PHCs readiness for the implementation of a new EHRS. The Saudi MoH is planning to implement an EHRS in all PHCs supervised by the $\mathrm{MoH}$ $(\mathrm{n}=2259)$. As illustrated in Chapter two, healthcare organisations have to be ready for change prior to EHRS implementation (Doebbeling et al., 2006; Lorenzi et al., 2004; Yusof \& Aziz, 2015; Yusof et al., 2008). An organisation's readiness for change was found to be an important factor in overcoming the potential challenges and for assuring smooth implementation of EHRS (Amatayakul, 2005; Jennett et al., 2003; O'Connor \& Fiol, 2006) (see Chapter Two). Readiness assessments are crucial to any EHRS implementation, because failure to consider readiness assessment has contributed to the failure of up to half of large organisational change attempts (Weiner, 2009). Furthermore, the purpose of conducting readiness measurement is to assess the preparedness of the healthcare organisations to introduce a new EHRS. Consequently, doing so will assist the project team in identifying any issues or requirements that need to be addressed during implementation (Biruk et al., 2014).

For improved, broader readiness assessments, factors from different perspectives, such as organisational, technological and human factors, should be included (see Chapter Two). According to Snyder-Halpern (2002), eight different themes need to be considered when assessing a healthcare organisation's readiness for EHRS implementation. These are: resources, end-users, technology, knowledge, processes, values and goals, management and structures, and administration support. These eight themes represent the factors identified in the literature review.

On the other hand, this study endeavoured to address some of the detected gaps in the literature, such as the role of CM on EHRS implementation and the provision of FR (see Chapter Two). As illustrated in the literature review (see Chapter Two), leadership and management were identified as influential factors in EHRS implementation. In addition, the cost of the implementation project was found to be an influential factor. Therefore, this study explored the impact of CM and FR on EHRS implementation in Saudi PHCs. This quantitative research used a self-administered, paper-based, close-ended 
questionnaire. The questionnaire was developed for people engaged in EHRS implementation in PHCs, and subsequently merged in one study and data collection instrument. This can assist in improving the response rate and avoiding time consuming procedures when conducting them separately. The objectives of this study are therefore:

- To assess PHCs readiness for EHRS implementation from the project team perspective.

- To evaluate the impact of Financial Resources (FR) and Centralisation Management (CM) on the implementation of the EHRS in PHCs in SA.

\subsubsection{Site}

This study was carried out at the Saudi Ministry of Health $(\mathrm{MoH})$, which is based in the Ministry headquarters in Riyadh, the capital city of the Kingdom of Saudi Arabia. The Saudi MoH manages and oversees healthcare organisations in Saudi Arabia (see Chapter One). Based on the last statistical yearbook that was published by the Saudi $\mathrm{MoH}$, in 2013, the total number of employees was 430,096 (MoH, 2012).

\subsubsection{Population}

The study population comprises all project team members directly or indirectly involved in implementing a large-scale EHRS project in Saudi PHCs. These consisted, for example, of heads of relevant departments (IT and PHC departments), senior managers, IT engineers, and technicians. This population of participants within the Saudi $\mathrm{MoH}$ has varying backgrounds and experience, departments, occupations and genders. The target population was all project team members $(n=53)$.

\subsubsection{Sampling technique}

To reach the most appropriate subjects for this study (taking into consideration their involvement in the project implementation and knowledge they held about EHRS implementation in PHCs in SA), non-probability, purposive, snowball sampling was used (Bryman, 2012; Thompson, 2012). Purposive sampling is a strategy that is often used in qualitative research (Bowling, 2009), and is also beneficial for opinion and attitude based 
surveys (Thompson, 2012). The selected sampling technique is useful when the data needs to be collected from specific individuals and for a specific purpose. In this study, the selected individuals were people at the Saudi MoH who could provide accurate information about the EHRS implementation project.

Identification of the study sample was conducted over two phases. Initially, I visited the relevant departments at the Saudi MoH (IT department and PHC department). I provided these departments with a copy of the ethical approval and facilitator letters obtained from the Saudi $\mathrm{MoH}$ (see Appendix A) to allow access to details of the potential study population. I requested a list of names and contact details of project team members who were involved in the EHRS implementation. The lists contained only twenty-seven names. I spent time at the MoH familiarise myself with the EHRS implementation project. During this time, I held informal meetings with some of the participants to ensure their appropriateness for the study and also ask them if there were other members of the project team that were not identified on the lists, in particular those from outside the selected departments. I collected demographic information from participants to ensure they appropriately represented the population. A total of fifty-three participants were selected due to their involvement, knowledge, expertise and participation in EHRS implementation projects in PHCs in SA.

Although bias in purposive sampling is higher compared with probability sampling techniques, this study failed to determine the participants' departments. Since the participants came from two main departments, namely IT and PHC departments, the determination of departments may assist in reducing bias. However, I physically collected the questionnaires in person, and I can confirm that individuals from both departments participated and returned the questionnaires.

\subsubsection{Data collection instrument}

The method used in this study was a structured, self-administered questionnaire composed of pre-defined items and response options (Bowling, 2009; Dawson, 2009; Offredy \& Vickers, 2010). Questionnaires are economical and also require less time when compared to other methods. The structured process implemented here by the questionnaires ensured quicker and cost effective collection of large data (Polit et al., 
2002). Such a questionnaire also offered enhanced privacy (Reja et al., 2003). In addition, questionnaires are frequently used to assess healthcare organisations readiness to implement new EHRS (Gagnon, Attieh, et al., 2014; Yusif et al., 2017).

Upon review, the relevant literature questionnaires were found to be common and more effective than other methods of assessing healthcare organisations' readiness to implement an EHRS (e.g. Biruk et al., 2014; Pare et al., 2011; Saleh et al., 2016; Simon et al., 2008). According to Ajami et al. (2011); Gagnon, Attieh, et al. (2014), using such a questionnaire can lead to better insight and provide more valid findings. The questionnaire was found to be a useful tool to gain information from a larger sample and help to obtain a different perspective on PHC readiness for an EHRS. Comparing with other data collection methods used to assess healthcare organisation, such as semistructured interviews (Gagnon, Attieh, et al., 2014; Yusif et al., 2017), the questionnaire is less time consuming and also accessible to the majority of the selected population in this study. As a result, the questionnaire method was deemed to be an appropriate method to gather data from a project team perspective.

A specific questionnaire was developed to achieve the study objectives. Most of the items $(\mathrm{n}=48)$ in this questionnaire were identified from an existing questionnaire called "Organisational Information Technology/Systems Innovation Readiness Scale (OITSIRS)" (see Table 3.3.1) (Snyder-Halpern, 2002), that had previously been used to assess the readiness of six US hospitals in 2002 (Snyder-Halpern, 2002), and then used to assess three US community hospitals in 2006 (Snyder \& Fields, 2006). The OITSIRS questionnaire has also been identified as a useful tool to assess healthcare organisations readiness for the implementation of new IT (Anderson \& Aydin, 2006; Gagnon, Attieh, et al., 2014; Yusif et al., 2017). Moreover, Gagnon et al. (2014) reviewed the frequently used data collection instruments used to assess healthcare organisation readiness for the introduction of new technology, and suggested using OITSIRS. OITSIRS was found to be comprehensive and highlighted most readiness measurements suggested by the literature review (see Chapter Two). 
Table 3.3.1: Organisational Information Technology/Systems Innovation Readiness Scale sub-themes.

\begin{tabular}{|c|c|c|}
\hline $\begin{array}{c}\text { Sub- } \\
\text { Theme/reliability }\end{array}$ & Definition & Questions \\
\hline Resources/.83 & $\begin{array}{l}\text { IT/S } \\
\text { innovation } \\
\text { support } \\
\text { mechanisms. }\end{array}$ & $\begin{array}{l}\text { 1.Funding is adequate for completion of EHRS } \\
\text { implementation. } \\
\text { 2.The project budget includes } \\
\text { training/retraining costs. } \\
\text { 3.There is a good ratio of full-time in-house to } \\
\text { contract IT staff to support the project. } \\
\text { 4.The project budget is consistent with the } \\
\text { organisation's strategic plan. } \\
\text { 5.Project teams have included both technical } \\
\text { support staff and users. } \\
\text { 6.Good quality vendor support for the EHRS is } \\
\text { typically available. }\end{array}$ \\
\hline End-Users/.83 & $\begin{array}{l}\text { End-user } \\
\text { profile }\end{array}$ & $\begin{array}{l}\text { 1.Adequate training is available to support users. } \\
\text { 2.A core group of users (champions) is available } \\
\text { to support implementation } \\
\text { 3.Users are typically involved in EHRS } \\
\text { implementation. } \\
\text { 4.Most users have an adequate level of computer } \\
\text { literacy. } \\
\text { 5.Users are typically supportive of EHRS. } \\
\text { 6.User competencies are appropriately } \\
\text { incorporated into job performance criteria. }\end{array}$ \\
\hline Technology/.83 & $\begin{array}{l}\text { IT/S } \\
\text { infrastructure } \\
\text { (e.g. hardware, } \\
\text { software, } \\
\text { networks, } \\
\text { writing, and } \\
\text { system } \\
\text { integration). }\end{array}$ & $\begin{array}{l}\text { 1.Research and development activities to learn } \\
\text { about new technology are supported. } \\
\text { 2. Development of information systems is based } \\
\text { on current market trends. } \\
\text { 3. There is a good fit between organisational and } \\
\text { EHRS implementation strategic plans. } \\
\text { 4.There are good quality vendor contracts } \\
\text { 5.Current work practices are adequately } \\
\text { supported by existing information systems. } \\
\text { 6.EHRS project implementation timeframes are } \\
\text { usually adequate. }\end{array}$ \\
\hline Knowledge/.78 & $\begin{array}{l}\text { Organisational } \\
\text { knowledge of } \\
\text { external and } \\
\text { internal } \\
\text { driving forces } \\
\text { influencing IT } \\
\text { innovation. }\end{array}$ & $\begin{array}{l}\text { 1. There is a lot of knowledge about the on-going } \\
\text { development needs of EHRS support staff. } \\
\text { 2. Knowledge about how EHRS implementation } \\
\text { is being used by other organisations is available. } \\
\text { 3. There is a lot of knowledge about EHRS } \\
\text { operational and capital budget trends. } \\
\text { 4. Historically, strategic and EHRS } \\
\text { implementation goals have been integrated. } \\
\text { 5. Administrators are very knowledgeable about } \\
\text { EHRS based on their past experience. }\end{array}$ \\
\hline
\end{tabular}




\begin{tabular}{|c|c|c|}
\hline & & $\begin{array}{l}\text { 6. In the past, EHRS users have been included in } \\
\text { decision-making processes. }\end{array}$ \\
\hline Processes/.85 & $\begin{array}{l}\text { Organisational } \\
\text { processes that } \\
\text { influences IT } \\
\text { innovation. }\end{array}$ & $\begin{array}{l}\text { 1.EHRS implementation needs are routinely } \\
\text { incorporated into the organisation's business } \\
\text { processes. } \\
\text { 2. The most appropriate individuals are involved } \\
\text { in the development of the EHRS implementation } \\
\text { strategic plan. } \\
\text { 3. Adequate communication mechanisms exist } \\
\text { to support shared communication across all } \\
\text { organisational levels. } \\
\text { 4. Effective mechanisms are in place to evaluate } \\
\text { EHRS implementation. } \\
5 \text {. Process improvement mechanisms are used } \\
\text { effectively to identify work process redesign } \\
\text { needs. } \\
6 \text {. EHRS implementation decision-makers are } \\
\text { adequately represented on key organisational } \\
\text { committees. }\end{array}$ \\
\hline $\begin{array}{l}\text { Values and } \\
\text { Goals/.89 }\end{array}$ & $\begin{array}{l}\text { Individual and } \\
\text { organisational } \\
\text { values and } \\
\text { goals } \\
\text { supportive of } \\
\text { IT/S } \\
\text { innovation. }\end{array}$ & $\begin{array}{l}\text { Individuals have a positive attitude toward } \\
\text { EHRS implementation. } \\
\text { There is a willingness to engage in the EHRS } \\
\text { implementation process. } \\
\text { There is an emphasis on the importance of } \\
\text { collaborative interdisciplinary teams to support } \\
\text { EHR implementation. } \\
\text { There is satisfaction with the contribution that } \\
\text { EHRS has made to the organisation. } \\
\text { There is a willingness to act on work process } \\
\text { improvement recommendations. } \\
\text { There is an openness to different perspectives } \\
\text { about EHRS implementation. }\end{array}$ \\
\hline $\begin{array}{l}\text { Management } \\
\text { Structures/.85 }\end{array}$ & $\begin{array}{l}\text { Organisational } \\
\text { management } \\
\text { structures and } \\
\text { operations that } \\
\text { influence IT/S } \\
\text { innovation. }\end{array}$ & $\begin{array}{l}\text { 1.The IT department effectively manages the } \\
\text { organisation's shared databases. } \\
\text { 2. The business structure supports involvement } \\
\text { of IS in strategic planning. } \\
\text { 3. Formal policies and procedures are available } \\
\text { to guide EHRS implementation processes. } \\
\text { 4. The IT strategic plan is an effective guide for } \\
\text { the organisation's EHRS implementation } \\
\text { processes. } \\
\text { 5. Formal communication mechanisms exist to } \\
\text { support user and IT support staff } \\
\text { communication. IT department reporting structure } \\
\text { 5. The IT } \\
\text { adequately supports IT staff. }\end{array}$ \\
\hline $\begin{array}{l}\text { Administration } \\
\text { support.92 }\end{array}$ & $\begin{array}{l}\text { Administrative } \\
\text { leadership and } \\
\text { practices } \\
\text { supportive of }\end{array}$ & $\begin{array}{l}\text { 1.Sufficient funds are available to support EHRS } \\
\text { implementation planning activities. } \\
\text { 2. Executives engage in mutual decision-making } \\
\text { with IT leaders regarding proposals and ideas. }\end{array}$ \\
\hline
\end{tabular}




\begin{tabular}{|l|l|l|}
\hline IT/S & 3. The top-ranking IT executive is regularly \\
innovation. & included in senior executive meetings. \\
& 4. EHRS implementation initiatives are usually \\
addressed as part of the organisation's overall \\
strategic planning. \\
5. Non-IT executives are routinely named as co- \\
sponsors for EHRS implementation projects. \\
6. Board members are actively engaged in key \\
EHRS implementation strategic plan \\
committees.
\end{tabular}

This information, provided to participants, was divided into three sections (see Appendix A). Firstly, there was a letter which described the nature of the study, the importance of their participation and their right to withdraw at any time. In addition, the letter explained how their responses would be treated and that their participation would be anonymous (for more details, see Section 3.2).

The second part asked for specific details regarding individual perceptions of PHCs readiness to the EHRS implementation, as well as the impact of CM and FR. This part was made up of sixty-eight items and was divided into three main themes. The respondent was required to answer the questions using a seven-point Likert scale response: Strongly disagree (1), Disagree (2), Somewhat disagree (3), No opinion (4), Somewhat agree (5), Agree (6), Strongly agree (7) (see Appendix A). Seven-point Likert scale response found to be useful when involve experts and other participants with wide knowledge about the studied context (Snyder-Halpern, 2002). Each of these themes were included to address the objectives of this study. The first theme aimed to assess PHC readiness for the implementation of the EHRS from the project team perspective. The second and third themes were related to the impact of CM on EHRS implementation in PHCs, and the impact of FR on the EHRS implementation in PHCs. These themes provided data that directly linked to the second objective of this study: "to evaluate the impact of FR and the CM on the implementation of the EHRS in PHCs in SA".

In order to determine the influence of CM on EHRS implementation, questions 49 to 57 of the survey asked about the respondent's perception of the influence of this type of management on EHRS implementation. The cost was identified in the literature review as being a barrier to these projects (see Chapter Two). There has been little research, however, about the influence of an abundance of FR as a facilitator for EHRS 
implementation. Therefore, the final part of the survey was comprised of eleven questions, from 58 to 68 , which related to the respondent's perception regarding the influence of FR on EHRS implementation in PHCs. The third and final part of the questionnaire collected information about the participant's demographic details. This asked about the participant's gender, position, their role in the EHRS implementation project, and if they had been involved in any similar projects before. The demographic information was used to describe the sample in order for inferences regarding the generalisability of the findings to be made.

\subsubsection{Questionnaire development}

The majority of the questions included in this data collection instrument were taken from a pre-existing questionnaire (see Section 3.3.4). However, as some modifications were made and additional items added, I conducted several preliminary procedures in order to assess and, if necessary, improve the questionnaire. The development of the questionnaire was carried out over two phases:

\section{Phase One}

The preliminary instrument was reviewed by two different expert panels. The first panel consisted of the supervisory team. The second panel consisted of external experts, including:

- two IT specialists from the headquarters of the Saudi MoH;

- the heads of the IT departments of two different hospitals;

- one academic from King Saudi University (who held a PhD in Health Informatics);

- one radiologist;

- one pharmacist (who held a master's degree in Health Informatics).

The purpose of the expert panels was to review the preliminary instrument, to check the if the questions being understandable, and to assess the content of the questions to ensure that all included items were relevant to the target population and addressed the study objectives. 
Both panels of experts suggested adding some demographic questions such as participant gender, and level of involvement (i.e. whether it was direct or indirect). In addition, they suggested to reduce the Likert scale responses from eight to seven where "no opinion" had one option instead of two.

\section{Phase Two}

Following a review and feedback on the questionnaire by the expert panels, it was sent to a small number $(n=5)$ of the project team as a pilot study to ensure that the questionnaire was clear, understandable and reliable. The data collection questionnaire was piloted at the headquarters of the Saudi MoH with volunteer members from the project team who had agreed to take part. Certain criteria were taken into consideration, such as different position level (e.g., supervisors, directors and senior managers), different departments, and different nationalities.

The aim of the pilot study was to gain feedback from the participants about the quality questionnaire such as readability, comprehensiveness, appropriateness and clarity. The pre-test draft of the questionnaire included questions at the end to assess what participants thought about it:

1. Did you understand the questions?

2. Were there any questions you did not understand and why?

3. Are there any questions you think should have been included?

4. Were there any questions you think should have been excluded?

5. Any comments.

Each participant in the pilot study completed the first draft of the questionnaire and also provided comments and feedback about the process and measures, the questionnaire administration time, and the clarity of the questions. Based on the responses from the pilot, none of the participants suggested including or excluding any questions. Overall, the pre-test pilot showed that the questionnaire was relatively clear and easy to complete. The pre-test was conducted over a period of two weeks, from $15^{\text {th }}$ September 2014 to $22^{\text {nd }}$ September 2014. As a result of the pilot feedback, minor spelling and grammar modifications were made to improve the clarity and readability of the questionnaire. In addition to the grammar and spelling mistakes, one duplication in the included items and 
numbering issues was detected by the volunteers. Since the modifications made were relatively minor, responses from volunteers were included in the final results.

\subsubsection{Data collection process}

Once the data collection instrument had been developed and was ready to be used, I started to think about the most appropriate method to distribute it. The first consideration was how to increase response rate and encourage participants to take part in this study. Therefore, the initial step in the data collection process for a questionnaire study is to decide whether to use an electronic or paper-based questionnaire. Each method has advantages and disadvantages. I decided to use the paper-based version due to the characteristics of the target participants. The majority of the potential participants in this study were occupying senior positions (e.g., senior managers and heads of departments). To improve the response rate, I had to encourage participants by visiting them in person and providing them with a copy of the questionnaire with an invitation letter attached. It was felt that using a paper-based questionnaire was better than sending an electronic copy by email and would ensure a greater engagement and completion rate. In addition, during the visit I described the importance of the research and the value of their participation. Although arranging to meet key people was difficult, I spent a lot of time to ensure that all relevant individuals received a copy of the questionnaire. Some participants did however receive a copy of the questionnaire via their personal assistant. Despite the challenges of distributing a paper-based questionnaire, this did not prove overly onerous, as all the participants were located in one place.

The questionnaire was distributed during the week of October 06, 2014. Fifty-three surveys were distributed to participants. Two "in person" reminder visits to the participants were undertaken after the distribution of the questionnaire. One took place during the week of October 20,2014, and the other took place during the week of November 03, 2014. Between these visits, phone calls were made to remind the participants to complete the questionnaire, either directly or via their personal assistant.

\subsubsection{Data analysis}

All data from the questionnaire was coded in numerical categories into SPSS Version 22 (Field, 2013). Firstly, a Cronbach's alpha test was used to examine the data collection 
reliability (see Chapter Four for more details). Thereafter, an initial descriptive analysis of the questionnaire data was undertaken. According to Taylor (2005), descriptive statistics are required to show quantitatively how a specific attribute is distributed within a population. Moreover, the purposes of conducting the descriptive analysis was to obtain a full description of the gathered data that included responses to the demographic questions and the main questionnaire. Descriptive analysis was used to display the data obtained from the participants. Due to the nature of the data obtained from the questionnaires, which was classified as categorical ordered data, I used the median instead of mean to display the data (Field, 2013).

The number of participants responding to each Likert response and percentages were included in the descriptive statistics. These responses were used to calculate a total agreement percentage for each question. The total agreement percentage was used to determine which items, within a given, scale had the highest level of agreement. The total agreement percentage was calculated by summing all those responding positively to a specific question (i.e. somewhat agree, agree, or strongly agree). The total agreement percentage scores were then ranked, in order based on the total agreement within the scale. This can assist with ranking those items that have the same median. 


\subsection{Study Two: Assessing PHC readiness for EHRS implementation in SA from a PHC staff perspective}

This study complements Study One, which was conducted to address the objective, "To assess PHC readiness for EHRS implementation from a project team perspective". Although, this study had the same objective, it was directed to a different population and utilised a different data collection instrument to comply with the addressed population. Therefore, the objective of this study was "To assess PHC readiness for EHRS implementation in SA from a PHC staff perspective”. According to Negash et al. (2018), employee perspectives are important when assessing organisation readiness to change. Although organisation readiness assessment documented to be influential factor to the EHRS implementation (see Section 3.3), some previous evaluation studies have failed to include aspects related to readiness at an individual level, such as individual attitude, awareness and knowledge about the EHRS (Biruk et al., 2014). This study therefore aimed to demonstrate the level of individual readiness to the EHRS implementation.

\subsubsection{Population and sampling technique}

The study population were all clinical staff such as physicians and administrative staff, such as receptionists, working in PHCs in SA that had not yet implemented EHRS ( $n=38514)$ (MoH, 2012). These staff were from various backgrounds, age groups, and occupations as well as genders.

The sampling strategy applied in this study was a multi-stage cluster sampling technique (Daniel, 2011; Levy \& Lemeshow, 2013). The Saudi MoH divided SA into thirteen regions (MoH, 2012). Therefore, at Stage One, I utilised the same division adopted by the Saudi MoH, with regions converted to clusters (see Table 3.4.1) (MoH, 2012). In the second stage, simple random sampling based on the geographical location of each province was used (Bryman, 2012; Thompson, 2012), PHCs in SA are similar to each other (see Chapter One), geographical location is the only difference between them. For instance, Makkah province was selected to represent the west side of the country, and Albaha selected to represent the south side of the country (see Table 3.4.1). As a result, five of thirteen regions were selected for this study (see Table 3.4.1). Simple random sampling is very useful in reducing sampling bias, as it allows the selection of a more 
representative sample (Bryman, 2012; Thompson, 2012). At Stage Three, a total of 21 out of 2259 PHCs were randomly selected within the five chosen clusters. The sample $(n=491)$ was drawn from the selected 21 PHCs, across the five selected regions.

Table 3.4.1 Main regions in Saudi Arabia and the number of PHCs in each province

\begin{tabular}{|c|c|c|c|c|c|}
\hline NO & Region & $\begin{array}{l}\text { Geographical } \\
\text { location }\end{array}$ & $\begin{array}{l}\text { Number } \\
\text { of PHCs }\end{array}$ & $\begin{array}{l}\text { Selected in } \\
\text { this study }\end{array}$ & $\begin{array}{l}\text { Number of } \\
\text { selected } \\
\text { PHCs }\end{array}$ \\
\hline 1 & Riyadh & East & 435 & Yes & 6 \\
\hline 2 & Gassim & Centre & 159 & Yes & 4 \\
\hline 3 & Makkah & West & 355 & Yes & 5 \\
\hline 4 & Almadinah & West & 154 & No & \\
\hline 5 & Alsharqiah & East & 248 & No & \\
\hline 6 & Albaha & South & 101 & Yes & 3 \\
\hline 7 & Asir & South & 317 & No & \\
\hline 8 & Najran & South & 65 & No & \\
\hline 9 & Hail & North & 100 & No & \\
\hline 10 & Alshamaliyah & North & 45 & No & \\
\hline 11 & Jazan & South & 155 & No & \\
\hline 12 & Tabuk & North & 73 & No & \\
\hline 13 & Aljouf & North & 52 & Yes & 3 \\
\hline Total & 13 & & 2259 & & 21 \\
\hline
\end{tabular}

\subsubsection{Data collection instrument}

Although methods such as the questionnaire have been frequently used in similar previous literature (see Section 3.3.4), this study used a modified data collection instrument to comply with the study objective and match the population background. The utilised questionnaire-based method was also used for the first time to assess implementation of an EHRS in SA PHCs. Initially, the questionnaire was presented in two languages (English and Arabic) for two main reasons (see Appendix A). The main language used in Saudi healthcare organisations is English, but the majority of employees in these organisations are Arabic native speakers $(\mathrm{MoH}, 2012)$. Therefore, for the sake of 
convenience and for the purposes of clarity and readability, the questionnaire was developed in both English and Arabic.

The questionnaire consisted of four main sections (see Appendix A). The first section contained a letter of assurance (for more details see Section 3.2). As illustrated in Chapter Two, individual readiness assessments can be conducted by including seven criteria: computer skills, gender, attitudes toward the implementation of the EHRS, knowledge about the EHRS implementation (Biruk et al., 2014; Yusif et al., 2017), experience at work, age (Yusif et al., 2017), Therefore, the second section was designed to obtain the participants' demographic data. Moreover, as revealed in Chapter Two, awareness about the perceived usefulness of the system found to be core predictor to the level of individual readiness to the introduction of new EHRS (Biruk et al., 2014; Kuo et al., 2013; Simon et al., 2008; Yusif et al., 2017). Therefore, the first set of questions in the third section assessed whether practitioners in PHCs were fully aware of the benefits of an EHRS. This scale contains thirteen items, which represent the benefits of the EHRS (see Section 3.5.3). Moreover, the third section comprised specific questions that were designed to assess PHCs readiness for the implementation of the system. Therefore, the second set of questions $(n=13)$ in the third section measured the PHCs readiness for implementation from the PHCs staff perspective. The questions used in this section were taken from the OITIRS questionnaire (see Section 3.3.4). Both scales are designed to gather responses on a 5-point Likert scale that ranged from Strongly disagree to Strongly agree, as follows: Strongly disagree (1); Disagree (2); Agree (3); Strongly agree (4); and No opinion (5). Five-point Likert scale was found to be less confusion especially for non-experts and those who have less knowledge about the studied context. In addition, 5-point Likert scale can increase the response rate (Buttle, 1996). Hence, the panel of experts who reviewed this questionnaire suggested to reduce the Likert scale from 7-points to 5-points (see next section for more details)

To achieve a better understanding of organisational readiness, user resistance and willingness should be measured (Lennon et al., 2017; Yusif et al., 2017). Therefore, the fourth section included two closed-ended questions. The first closed-ended question asked whether participants were enthusiastic about the implementation of the EHRS. The second closed-ended question asked participants whether they would resist the implementation of the EHRS in their workplace. Part of readiness evaluation is to 
determine the specific requirements of individuals who will use the EHRS, so the system can be designed with consideration of their specific needs (see Chapter Two). Therefore, an additional open-ended question asked participants to list any personal requirements related to the implementation of the EHRS and any recommendations they could suggest for improving it.

\subsubsection{Questionnaire development}

Similar to the development procedures adopted in Study One, due to modifications and additional items, the questionnaire of this study was also developed in two phases. In Phase One, the preliminary data collection instrument was reviewed by the same expert panels, however, additional experts joined the second panel. This consisted of three IT engineers from the headquarters of the Saudi MoH, the general manager of the PHC department at the $\mathrm{MoH}$, five heads of IT departments from five different hospitals, three academics from a Saudi university who held a $\mathrm{PhD}$ in Health Informatics, three radiologists, and one pharmacist who held a Master's degree in Health Informatics. As a member of the Health Informatics Club (HIC) in SA, I benefited from the experience of other members. The HIC in SA created a WhatsApp group, which currently has more than 200 active members from different healthcare organisations and backgrounds in HI. I selectively invited some of the members to review the preliminary instrument to ensure that all included items were relevant to the target population and that they addressed the study objectives.

One of the main comments, by the panel of experts, was the recommendation to distribute the questionnaire in two languages (Arabic and English). Once the questionnaire was developed in both languages, it was sent to two Saudi PhD students of translation at Swansea University, who reviewed the questionnaire before the final draft was distributed to the participants. They also suggested removing all items that were not relevant to the target population $(n=35)$. The deleted items were concerned with factors related to the organisation, such as project team selection, software selection, and planning (see section 3.3.4). The panel of experts believed that the current study population may not be able to accurately respond to these items, due to lack of awareness caused by the type of project management utilised (see Chapter One). 
In the second phase, sixteen volunteers agreed to take part in the pilot study. The aim of the pilot study was to gather feedback regarding the questionnaire design before the research was undertaken on the required population. Certain criteria were taken into consideration, such as differences in gender, occupation and nationality of the participants, to ensure all items were relevant and that both languages were clear and readable for all participants. Similar to Study One, the pre-test draft of the questionnaire also included questions at the end to gain feedback about the questionnaire (see Section $3.3 .5)$.

Each volunteer completed a first draft of the questionnaire and provided comments and feedback about the process and the measures, the questionnaire administration time, and the clarity of the questions. None of them asked to include or exclude any question. Overall, the pre-test showed that the questionnaire was relatively clear and easy to fill out. The pre-test was conducted over a period of two weeks, dating from $14^{\text {th }}$ December 2015 to $7^{\text {th }}$ January 2016 . Since very minor modifications were made to the data collection instrument based on this pilot study, all responses obtained from pilot study were included with actual findings. Both SPSS files that included pilot study findings and final study findings were merged into one SPSS file. A Cronbach's alpha test revealed that questionnaire used in this study was statistically reliable.

\subsubsection{Data collection process}

Similar to Study One, once the data collection instrument had been developed and was ready to be used, I started to think about the most appropriate method to distribute it. The first consideration was how to increase the response rate and encourage participants to take part in this study. Initially, I used the electronic questionnaire because it was found to be an effective tool to assess the readiness of numerous PHCs compared with other methods such as observation. As mentioned earlier, twenty-one PHCs were selected for assessment in the current study, for generalisability purposes (see Section 3.4.1). On the other hand, the decision regarding the method of distribution of the questionnaire for this study was based on three main factors. Firstly, the geographical challenges, the Kingdom of Saudi Arabia is a huge country. It was impossible to physically distribute a paper-based questionnaire to all the selected PHCs, as postal services in SA are relatively poor. 
Secondly, the sample size was large, and therefore, using a paper-based questionnaire would have been very expensive. Finally, I conducted this study from the UK, so it was impossible to physically distribute the questionnaire. Therefore, I decided to utilise an online self-administrated questionnaire via Survey Monkey to collect the data. The decision to select Survey Monkey over Google forms or other websites was that the Medical School at Swansea University has a premier account which allows researchers to create and design more flexible and advanced surveys and then export the responses to SPSS.

The questionnaire distribution of this study was conducted over a period of ten weeks, from $11^{\text {th }}$ January 2016 to $31^{\text {st }}$ March 2016. Two reminder e-mails were sent to the participants after distribution of the initial questionnaire. The first e-mail was sent during the week of $8^{\text {th }}$ February 2016, and the second e-mail was sent during the week of $7^{\text {th }}$ March 2016. The official staff emails were not effectively used, the majority of the selected PHCs staff were still using their personal e-mail address at work which was not accessible to me. Therefore, I was directly linked with representatives from the twentyone selected PHCs. Each one of these representatives was provided a copy of the ethical approval letter and was then invited to join a 'WhatsApp' group which was created by me for this purpose. All twenty-one representatives accepted the invitation. Once all of them joined the group, I sent a unique link obtained from surveymonkey.com to the group and asked them to fill in the questionnaire and then forward it to all other staff in the selected PHCs via their personal emails or other possible communications such as other WhatsApp groups.

\subsubsection{Data analysis}

Similar to the data analysis procedures performed in Study One (see Section 3.3.7), the data were analysed using SPSS V.22. Firstly, the scales of the study were tested for reliability using a Cronbach's alpha test to ensure the consistency of the data collection instrument (see Chapter Five for more details). Thereafter, descriptive analysis was applied to display the data obtained from the participants using median, percentages, total agreement and rank (except for the barriers scale, which didn't include total agreement). These responses were used to calculate a total agreement score for each question. Each question was ranked based on the level of agreement, from the highest agreement to the 
lowest. The total agreement was combined (agree + strongly agree) to enable me to distinguish between items, i.e. to see which of the items generated more agreement compared to the others (as ranked).

Following the presentation of descriptive data, inferential statistical tests were used to test any a priori hypotheses. All tests are non-parametric and were chosen as a result of the data being considered of an ordinal nature and nominal. Differences tests were performed using a Mann-Whitney U test for two groups and a Kruskal Wallis for three groups, or more, to examine the differences between groups and then determine if demographic differences influenced the level of readiness of those individuals (see Section 3.4.2).

\section{$\underline{\text { Responses to open-ended questions }}$}

Responses to the open-ended questions were presented and organised using Microsoft Excel. All responses were exported as text from surveymonkey.com to a Microsoft Excel sheet. Arabic responses were translated to English by me and then checked by a professional translator. I read the responses obtained by the participants. The responses were then grouped into themes where similar responses were gathered under one theme. All themes were then coded to label each response with the appropriate code to allow Excel to calculate the number of responses in each theme. Finally, all responses were presented in a table, which included the themes, an example of a response to each theme, and the number of responses to each theme. 


\subsection{Study Three: Evaluation of implemented EHRS in PHCs in SA from the perspective of end-users}

Since this thesis was aiming to explore the large-scale implementation of an EHRS in PHCs in SA, I decided to conduct a further study to evaluate the currently implemented EHRS, because assessing PHC readiness for implementation alone is therefore not sufficient to achieve this aim. Although three questionnaire-based studies were conducted in this thesis, this study focuses on the previously implemented EHRS in PHCs in SA (see Chapter One), whereas the two previous questionnaire-based studies were conducted to assess PHC readiness for an EHRS implementation project.

As revealed in Chapter One, previous implementations of an EHRS in Saudi PHCs have failed. Therefore, it was beneficial to investigate the barriers that may have contributed to the failure of these projects. If these barriers are overcome in future, this may enhance the chance of success of future projects. Other questionnaire-based studies focus on the readiness of PHCs for new EHRS implementation projects.

To achieve the study objective, this study also attempted to determine the barriers and facilitators to EHRS implementation. Moreover, to achieve a better understanding of the implemented EHRS, end-user satisfaction was examined. EHRS end-user satisfaction surveys have been identified as useful tools to measure the success or failure of any EHRS implementation (Yusof et al., 2008). Consequently, several studies have examined enduser satisfaction with EHRS since 1996 (Bani-Issa et al., 2016; Khajouei et al., 2011; Lee et al., 1996).

In addition to EHRS end-user satisfaction, all other factors identified in the literature related to the organisation, individual and technology were addressed and evaluated as influential factors. Moreover, to achieve its aim, this study examined the relationship between different factors that influence EHRS implementation, such as the relationship between perceived usefulness and training (Carr et al., 2010) (see Chapter Two). This questionnaire-based study utilised both closed-ended and open-ended items. 


\subsubsection{Site of the study}

This study was carried out at PHCs that had previously implemented an EHRS. These PHCs were distributed across thirteen regions in Saudi Arabia (see Table 3.5.1) (MoH, 2012).

\subsubsection{Population and sampling technique}

In the current research work, the eligible population involved all end-users of EHRS within 150 PHCs in Saudi Arabia. These end-users within the Saudi MOH came from various backgrounds, age groups, departments and occupations, as well as genders. Such a population was selected since they were currently using or had been using an EHRS in their workplace. According to documents attached to an email sent by the IT department at the Saudi MoH, only 150 PHCs had fully implemented EHRS in SA (will be explored in Chapter Seven).

The sampling strategy applied in this study was similar to the one adopted in Study Two. The number of PHCs with EHRS varied from province to another. For instance, in Riyadh there are twenty-one PHCs with an EHRS, while Aljouf has eight. In Stage three, a total of twenty-one out of 150 PHCs were randomly selected from within the five chosen clusters (see Table 3.5.1). Finally, the sample $(n=483)$ was drawn from the selected twenty-one PHCs across the five selected regions. 
Table 3.5.1: Regions of Saudi Arabia and number of PHCs in each region

\begin{tabular}{|c|c|c|c|c|c|}
\hline NO. & Region & $\begin{array}{l}\text { Geographical } \\
\text { location }\end{array}$ & $\begin{array}{l}\text { Number of } \\
\text { PHCs with } \\
\text { EHRS }\end{array}$ & $\begin{array}{l}\text { Selected in } \\
\text { this study }\end{array}$ & $\begin{array}{l}\text { Number of } \\
\text { selected } \\
\text { PHCs }\end{array}$ \\
\hline 1 & Riyadh & East & 21 & Yes & 5 \\
\hline 2 & Gassim & Centre & 12 & Yes & 4 \\
\hline 3 & Makkah & West & 16 & Yes & 4 \\
\hline 4 & Almadinah & West & 11 & No & \\
\hline 5 & Alsharqiah & East & 10 & No & \\
\hline 6 & Albaha & South & 9 & Yes & 4 \\
\hline 7 & Asir & South & 13 & No & \\
\hline 8 & Najran & South & 11 & No & \\
\hline 9 & Hail & North & 9 & No & \\
\hline 10 & Alshamaliyah & North & 11 & No & \\
\hline 11 & Jazan & South & 10 & No & \\
\hline 12 & Tabuk & North & 9 & No & \\
\hline 13 & Aljouf & North & 8 & Yes & 4 \\
\hline Total & 13 & & 150 & & 21 \\
\hline
\end{tabular}

I visited the head of the PHC department at the Saudi MoH headquarters and provided him with a copy of the ethical approval and facilitator letters. The head of the department then linked me with a small number of PHCs to facilitate the process of distribution of the questionnaires. According to the list obtained from the PHC department, the number of PHCs in each province with EHRS varied from eight to twenty-one, depending of the size of the province and number of residents.

All potential participants in these areas were sent an electronic copy of the questionnaire. The following section highlights the data collection process and describes the questionnaire distribution mechanism. 


\subsubsection{Data collection instrument}

The utilisation of a questionnaire to evaluate EHRS implementation is not unique to this study; as revealed in the literature review (see Chapter Two) the questionnaire was the most frequently used in the field of EHRS implementation (Gagnon, Ghandour el, et al., 2014; Hor et al., 2010; Khajouei et al., 2011). Moreover, Nguyen et al. (2014), in their systematic review, found that the most frequent and effective way to evaluate EHRS implementation is through "perception-based data collection" via the utilisation of questionnaire-based research. According to Hayrinen et al. (2008), there have been twenty-eight questionnaire-based research studies conducted to evaluate EHRS implementation. Although the questionnaire was frequently used in previous literature, this study employed modified data collection instruments used for the first time to evaluate EHRS implementation in Saudi PHCs.

In this section, I describe in detail the design of the questionnaire and its content. I developed the questionnaire after reviewing the relevant literature. Similar to Study Two, the questionnaire utilised in this study was available in both English and Arabic for the same reasons mentioned in Study Two. The questionnaire was designed for the people who were using EHRS in the Saudi PHCs. The aim of this study was therefore to evaluate the EHRS implemented in PHCs in SA.

This questionnaire was divided into three sections (see Appendix A). The first section contained a letter of assurance. The second section of the questionnaire sought information about the participant's demographic details, specifically gender, age, position, years of experience in current workplace, years of experience using a PC, years of experience using an EHRS, the name of their PHC, region name, and if they were still using an EHRS. These demographic details were included in the questionnaire because they were identified in the literature as potential influencing factors of EHRS end-user satisfaction (see Chapter Two). Due to this importance, several studies were keen to include gender and age as essential questions in their data collection instruments to assess the level of EHRS end-user satisfaction and acceptance of system implementation and use (e.g. Bani-Issa et al., 2016; Biruk et al., 2014; Hamid \& Cline, 2013; Pare et al., 2008; Saleh et al., 2016). 
The third section of the questionnaire was divided into three main themes. In this section, a total of sixty-four questions were included in order to evaluate the implemented EHRS, determine the main barriers and facilitators, and to establish end-user satisfaction and attitudes towards the use of EHRS. More than half of the items $(n=37)$ were taken from a pre-existing questionnaire called "The Clinical Information System Implementation Evaluation Scale (CISIES)" (Gugerty et al., 2006). Additional items (n=27) were added to fully achieve the objectives of the study. The CISIES has been widely used in the field of ICT implementation in healthcare, and has already been implemented in various similar studies. CISIES can gather user attitudes on health information systems, and was frequently used in similar studies conducted in various countries. For instance, Hsieh et al. (2009) implemented this tool in a Taiwan health centre to analyse user attitudes toward the Mobile Electronic Medication Administration Record System (ME-MAR). It has also been used in the USA by (O'Meara, 2007) to evaluate user attitudes toward a digital documentation system in Ambulatory Surgery Centres (ASCs).

Participants were asked to rate each question using a scale that ranged from Strongly Disagree to Strongly Agree, as follows: Strongly Disagree (1); Disagree (2); Neutral (3); Agree (4); and Strongly Agree (5). Items that were rated as strongly disagree or disagree represented dissatisfaction, and those that were rated as strongly agree and agree represented satisfaction with EHRS implementation (Gugerty et al., 2006). The items in CISIES focused on end-user perception of EHRS implementation and use, which assisted in providing sufficient understanding of the quality of the implemented EHRS. The decision to select CISIES was also made because it addressed all factors determined to influence EHRS implementation. The items in this section were categorised under four main themes. It began with seventeen questions querying the perceived usefulness of the EHRS, then further items $(n=29)$ were included to evaluate other factors such as training and support. Sixteen items were included to determine the barriers to EHRS implementation. Finally, two open-ended questions were added for further clarification and recommendations. Although, the majority of the items in this questionnaire were taken from pre-used questionnaire, findings from semi-structure interviews were taken into consideration. For instance, some items in the barriers scale were added based on emerged barriers from the semi-structure interviews. 


\section{Theme One: Overall attitudes towards and satisfaction with EHRS implementation}

In order to establish end-user satisfaction and attitudes towards the EHRS implementation, forty-six items were included, which were largely $(n=37)$ quoted from CISIES. This theme allocated several factors determined to be influential factors in EHRS implementation (see Chapter Two), some of which are directly related to EHRS end-user satisfaction. For instance, perceived usefulness and perceived ease of use are considered key indicators in measuring end-user acceptance of EHRS implementation (Devine et al., 2010). In addition, effectiveness and performance of EHRS implementation can influence healthcare practitioner satisfaction with the system (Khalifa \& Alswailem, 2015). The other nine items were added based on a suggestion made by previous literature to fully examine the perceived usefulness of the implemented EHRS (Altuwaijri, 2011; Bardhan \& Thouin, 2013; Chaudhry et al., 2006; Gajanayake et al., 2013; Mekhjian et al., 2002; Menachemi \& Collum, 2011).

\section{Theme Two: The barriers to EHRS implementation}

To assess the barriers to EHRS implementation, the "Barriers to EHR implementation" scale was used (see Appendix B). This consisted of sixteen questions developed according to the most frequent barriers identified in the literature review (see Chapter Two). The aim of this scale was therefore to determine the main barriers to the implementation of EHRS and to find which barriers had the most impact. It was anticipated that the responses offered may provide some insight and clarification regarding the barriers that could result in unsuccessful EHRS implementation in PHCs in Saudi Arabia. Participants were asked to rate each item using a scale that had three response options: not a barrier, minor barrier, and major barrier.

\section{Theme Three: General clarification and end-user recommendations regarding possible ways to improve EHRS implementation in PHCs}

The aim of adding open-ended questions was to gain further information about the implemented EHRS, as well as to determine the barriers to and facilitators of EHRS implementation in PHCs. Question Twelve required the participants to mention the features of EHRS they most liked. Question Thirteen required the participants to provide 
recommendations to improve EHRS implementation in PHCs. Part of the evaluation of the implemented EHRS was to gain further information via these open-ended questions. These two open-ended questions were an important addition to the questionnaire as they sought to obtain rich information and suggestions on how to help the EHRS project team make better decisions. Moreover, the purpose of asking open-ended questions was to encourage the participants to write full, meaningful answers in relation to their knowledge and opinions on improving EHRS implementation.

\subsubsection{Questionnaire development}

The majority of items, included in this study, were from existing questionnaires. These questions were included because of their comprehensiveness and relevance to the factors identified in the literature review. To ensure all factors identified in the literature review were examined in this study, a mind-map was developed to match the items covered by the questionnaire. Additional items were added to the questionnaire to achieve all objectives of this study. For instance, sixteen items were used to determine the main barriers to EHRS implementation in PHCs in SA. The new items were also imported to the mind-map to test their appropriateness in helping achieve the objectives of the study. Bryman (2012) suggested considering such hypotheses when designing new questionnaires. Thus, I took into account hypotheses from the literature review. For instance, the literature identified that participant demography (e.g., gender, age, and occupation) may influence their satisfaction with EHRS implementation (see Chapter Two). Therefore, I included all potential questions related to the participant demographic information to examine their impact on participant satisfaction.

Since this study was conducted at the same time as Study Two, similar development procedures were conducted to develop the questionnaire of this study (see Section 3.4.3 0 ). Although the same expert panels reviewed the questionnaire in the first phase of development, different feedback was received from them. The population of this study may not be able to provide all required information about the implemented EHRS, such as leadership and management, cost of the project, or project team selection and communication factors. Therefore, I asked the panel to review the questionnaire to make 
sure all questions were answerable by the target population and to avoid irrelevant responses which may affect the questionnaire's reliability.

Similar to Study Two, the panel of experts recommended to design the questionnaire to be available in both English and Arabic. Moreover, the barriers scale was ranked from major facilitator to major barrier, as follows: major barrier, minor barrier, not a barrier or facilitator, minor facilitator, major facilitator. However, the panel were of the opinion that a two-sided Likert scale may cause confusion in the participants. Therefore, the scale range was reduced from five to three (see previous section). In addition, since this data collection instrument was designed to evaluate the previously implemented EHRS, it was more beneficial to determine the barriers that led to failure of the project. Finally, a few spelling mistakes and translation errors were detected and corrected.

Phase Two: Following review and feedback on the questionnaire by the expert panels, it was sent to a small number $(n=13)$ of the implemented EHRS end-users in Saudi PHCs as a pilot study to ensure that the questionnaire was clear, understandable and reliable. Every participant involved in the pre-test was selected by me in coordination with the representatives of the PHCs by the utilisation of simple random technique (see Section 3.5.5). Volunteers from different occupations, genders, EHRS usage and nationalities were selected for the pilot study. A Cronbach's alpha test revealed that questionnaire used in this study is statically reliable. Responses obtained from the pilot study were added to the final findings.

\subsubsection{Data collection process}

Similar to Study Two, an online self-administrated questionnaire was used to collect data in the current study. Since I had no direct access to the PHCs, selection was made with the coordination of the PHC department at the Saudi MoH. Therefore, once the PHCs and target population had been identified, I distributed the questionnaire for this study in the same way as Study Two. Similar to Study Two, the Department of PHCs at the MoH provided me with the contact details of representatives from the selected PHCs to contact them directly and facilitate distribution of the questionnaire. All PHC representatives were invited to a WhatsApp group to fill in the questionnaire and then forward the questionnaire link to other staff members at the selected PHCs. All participants received 
an electronic copy of the questionnaire via a PHC representative (see 3.4 .4 for more detail).

\subsubsection{Data analysis}

The data analysis performed for this study was similar to that of studies One and Two. Initially, a reliability test was performed using a Cronbach's alpha test (see Chapter Six for more details), then descriptive analysis to display all obtained data. While demographic tables included frequency and percentages, both main scales include median, percentages, total agreement and rank. As proved earlier, individual satisfaction to EHRS implementation may be influenced by characteristics such as position, gender, experience with computers, etc. (see Section 3.4.2). Therefore, a Mann-Whitney U test was used to examine whether there are any differences between males and females in terms of level of satisfaction, as hypothesised in the literature. A Kruskal Wallis test was also used to examine whether there are any differences between nurses and other PHC staff in regard to their level of satisfaction, as hypothesised in the literature. The Kruskal Wallis test was used with other demographic variables that included more than two groups, such as age and experience using a PC, to determine whether these demographic differences influenced the level of satisfaction, as suggested in previous literature (see Chapter Two).

It was obvious from the literature that certain factors that influence EHRS implementation are associated. Whereas some of the study scales represent these factors, a Spearman's rho was used to determine any correlation between the main scales used in the study. According to Carr et al. (2010); and Escobar-Rodriguez and Bartual-Sopena (2013), factors such as perceived usefulness and perceived ease of use are associated with training and support (see Figure 3.5.1). Therefore, a Spearman's rho correlation coefficient test was used (Field, 2013) to examine whether there was a relationship between these factors, as revealed in previous literature (see Chapter Two). The procedures adopted to analyse the responses to open-ended questions were exactly the same as those adopted in Study Two. 


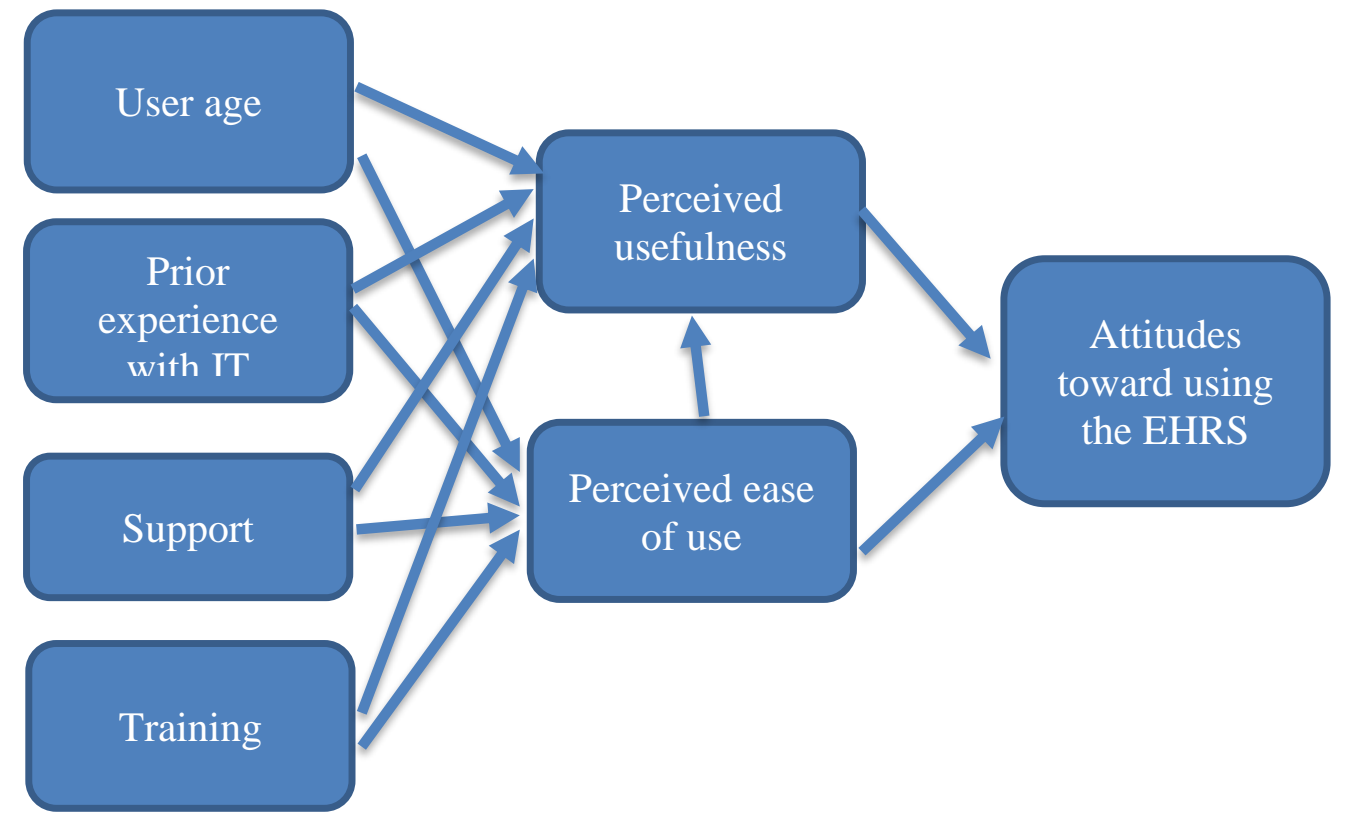

Figure 3.5.1: The relationship between factors influencing EHRS 


\subsection{Study Four: Semi-structured interviews to explore EHRS implementation in PHCs in SA from a project team perspective}

The aim of the thesis was to explore the large-scale implementation of EHRS in PHCs in SA. The decision to conduct semi-structured interviews was made based on the ability of interviews to provide in-depth, rich and detailed, information which cannot be obtained via questionnaire-based approaches. Therefore, to achieve this aim, further qualitative research was conducted via semi-structured interviews, and the validity of the findings was improved through the triangulation of different resources of data and the mixing of both qualitative and quantitative methods (Bryman, 2012).

Although, PHC readiness was assessed in Studies One and two, it was difficult to gain further information about certain pre-implementation procedures, such as project team selection. Therefore, this study provided additional and detailed information to allow a better understanding of previously examined aspects. In addition to readiness measurements, this study also sought further clarification of the impact of CM and FR, also previously examined in the study. This approach was previously termed sequential explanatory mixed-methods (Bryman, 2012). Barriers and facilitators to EHRS implementation were quantitively determined from an EHRS end-user perspective in Study Three. However, this study aimed to provide an in-depth description of the main barriers to and facilitators of EHRS implementation in PHCs from the project team perspective. Although the EHRS used in Saudi PHCs were quantitatively evaluated using questionnaire-based research (see Section 3.5), this study aimed to evaluate the EHRS using semi-structured interviews to gather in-depth descriptions of their implementation, the evaluation was based on a project team perspective.

The qualitative research comprised a set of face-to-face interviews with eleven members of the project team in the Saudi MoH. These members were involved in the planning and other implementation procedures. The interviews were semi-structured and were based on a series of open-ended questions using a pre-defined interview guide (see Appendix A). This study was conducted with the same population as Study One. 


\subsubsection{Study Sampling and population}

Policymakers and other project team members were identified as the most appropriate individuals to provide an in-depth description of EHRS implementation. Hartzler et al. (2013) suggested conducting one to one interviews with key informants from the project team to explore EHRS implementation. Silverman (2013) has suggested using purposive sampling for research that involves interviews, where a researcher gathers samples based on the requirements of the interview participants who are related to the research topic. The targeted population of this study comprised all members of the project team involved in the implementation of an EHRS in Saudi PHCs (see Section 3.3.2 for more details). The population for this study was exactly same as that of Study One. All participants $(n=53)$ from Study One were also invited to participate in an in-depth interview (see Section 3.3.6).

Out of 53 members of project team who involved in the EHRS implementation in the Saudi PHCs, eleven individuals were interviewed. The purposive sampling technique was selected, as key informants were required to ensure they had wide knowledge of the project. For instance, the majority of the items in the interview guide required the participants to provide details and in-depth information about the EHRS, and barriers and facilitators they faced during the implementation process. In this study, bias in purposive sampling has been reduced though conducting a sufficient number of interviews until no new information emerged (Bryman, 2012; Creswell, 2014).

\subsubsection{Data collection via semi-structured interviews}

The type of questions used were open-ended, in order to allow the participant the flexibility to describe their views and opinions (Bryman, 2012). Semi-structured interviews allow me to expand on the questions following unexpected or interesting responses (Mitchell \& Jolley, 2009). Semi-structured interviews can also gather a wider variety of detailed data (Miles \& Gilbert, 2005). Therefore, the main aim of conducting semi-structured interviews was to gain a comprehensive understanding and explanation of the full process of EHRS implementation in PHCs. 
This included a detailed plan of how certain procedures were to be implemented. For example, the methods used to provide training and support. In addition, semi-structured interviews were selected because they are more flexible and allow me to discuss additional issues that aren't covered in the interview guide. This allowed me to obtain insight into the perceptions and attitudes of the project team.

Similar studies have also utilised semi-structured interviews to explore the implementation of EHRS in different healthcare organisations and countries (e.g. Alkraiji et al., 2013; Ash et al., 2003; Boyer et al., 2010; Cresswell et al., 2012; Godoy \& Soares, 2017; McAlearney et al., 2014; McAlearney et al., 2013; Terry et al., 2009). Although, semi-structured interviews have not been the most favoured or frequently used approach for other research (see Chapter Two), the above-mentioned researchers were able to provide a detailed description and explanation of EHRS implementation. Their findings were found to be useful to researchers and policymakers alike. For instance, the findings yielded better decision-making regarding the provision of training and support (see Chapter Two). At the readiness assessment level, although qualitative methods such as semi-structured interviews have been used less than questionnaires (Gagnon, Attieh, et al., 2014; Yusif et al., 2017), they can assist in providing in-depth descriptions of readiness for EHRS implementation and other pre-implementation procedures. For instance, semi-structured interviews are able to provide in-depth descriptions of the implementation process, such as software selection, whereas closed-ended questionnaires simply provide yes or no responses (Bryman, 2012).

\subsubsection{Data collection process and the interview guide}

During October and November of 2014, eleven one-to-one interviews took place with the selected participants. The interviews were conducted over a two-month period. I scheduled up to two hours for each interview, however, no time limits were applied with regards to how long the interview could last. Participants were informed that they could withdraw from the interview at any time. In addition, in order to make the interviews as convenient as possible for the interviewees, I travelled to the participants to interview them. 
To save the participants' time, I prepared well in advance of the interviews and made sure to provide a clear and well organised interview guide. I sought permission to record the interviews and made sure that the recording devices had a full battery and sufficient storage space for the interviews. In addition, mobile phones were switched to airplane mode to prevent any phone calls or other notifications interrupting the interviews. I arrived at least thirty minutes prior to the interview time to make sure that the interview location was suitable. With the exception of one interview, all were conducted at the same location.

All the participants were presented with the same questions, in accordance with the guidelines of conducting interviews (see Appendix A). Robson (2011) has suggested recording interviews by audio, so that the information can been gathered accurately. The interviews were digitally recorded using an iPad and an iPhone. The memory available on both devices was capable of recording audio for up to fifty hours. Two devices were used to ensure that one source was always available if the other device was lost or damaged during the interview. Each device was kept opposite to the other to record the audio accurately. The voice recording files were uploaded to my laptop and checked to see if there were any obvious issues during the recording. All voice files were clear, which assisted the transcription of the interviews.

Field notes were taken during the interview, to avoid interruption when new questions emerged during the interview. This allowed me to generate more questions to overcome the omissions and also to clarify any comments made. This also allowed flexibility in the interview and helped the interviewer identify any comments that could lead to new questions and fields of research. In addition, some field notes were taken during the interview to capture body language and participants reactions that could not otherwise be detected in the audio recording.

I began the interview by introducing myself and giving a description of the nature of the research. I then explained why the participant had been invited to take part. I allowed sufficient time for the participants to read the consent form before starting to record the interview and ask questions. I confirmed with the participants that they were happy to take part in the study and asked them to sign the consent form. At the end of each interview, all participants were encouraged to raise any comments or issues that had not 
been addressed during the interview. The interviews lasted between 35 and 130 minutes, with a mean of around 65 minutes.

To achieve the objectives of this study, I took into account the main factors influencing EHRS implementation as determined by the literature during the creation of the interview guide (see Table 3.6.1). The interview guide therefore focused on general views on EHRS implementation and any barriers and facilitators that were associated with the implementation of large-scale projects. In order to gain a broad understanding of the issues related to large-scale EHRS implementation, I took into consideration both negative and positive views from the participants. The number of questions in the interview guide was the same for each interview, as all the participants were purposively selected and deemed to have a similar level of background about the studied context. 
Table 3.6.1: Interview guide based on the identified factors from the literature

\begin{tabular}{|c|c|}
\hline Factors & Questions from the interview guide \\
\hline Planning & $\begin{array}{l}\text { What are the main Elements included in the plan? } \\
\text { Can you please describe to me your plan to } \\
\text { implement the EHRs in the PHCs including } \\
\text { implementation timeline? } \\
\text { Can you please describe how you decided to } \\
\text { implement the EHRS in the PHCs? }\end{array}$ \\
\hline Readiness & $\begin{array}{l}\text { Can you please describe the level of the PHCs } \\
\text { readiness to implement the EHRS? } \\
\text { Was there any readiness assessment? } \\
\text { Do you think PHCs ready to the EHRS } \\
\text { implementation? Why? }\end{array}$ \\
\hline $\begin{array}{l}\text { Workflow } \\
\text { analysis and } \\
\text { redesign }\end{array}$ & $\begin{array}{l}\text { Did you consider the organisation structure and } \\
\text { workflow redesign to meet the new changes? }\end{array}$ \\
\hline $\begin{array}{l}\text { Teamwork } \\
\text { selection }\end{array}$ & $\begin{array}{l}\text { Can you please describe how you decided to select } \\
\text { the project team and what are the criteria you took } \\
\text { into account? } \\
\text { Was the champions involve in the project team? }\end{array}$ \\
\hline $\begin{array}{l}\text { Teamwork } \\
\text { communication }\end{array}$ & $\begin{array}{l}\text { What steps you take into consideration to ensure } \\
\text { adequate communication between project team in all } \\
\text { phases? }\end{array}$ \\
\hline $\begin{array}{l}\text { Leadership and } \\
\text { management }\end{array}$ & $\begin{array}{l}\text { Can you please describe the role of the leadership } \\
\text { and management in the implementation planning and } \\
\text { process? } \\
\text { Was there any involvement from seniors and top } \\
\text { managements in the EHRS implementation? Can } \\
\text { you please describe their role in the implementation? }\end{array}$ \\
\hline
\end{tabular}




\begin{tabular}{|c|c|}
\hline & $\begin{array}{l}\text { Do you think the CM influence the EHRS } \\
\text { implementation? how this type of management } \\
\text { influences the implementation of the EHRS in the } \\
\text { PHCs? } \\
\text { In your opinion, what are the main factors } \\
\text { influenced directly by the CM? } \\
\text { In which way the CM influence these factors? }\end{array}$ \\
\hline Training & $\begin{array}{l}\text { Was there training for end-users? } \\
\text { Can you please describe how you decided to } \\
\text { provide training to the end-users of the EHRS? }\end{array}$ \\
\hline $\begin{array}{l}\text { Technical and } \\
\text { financial } \\
\text { support }\end{array}$ & $\begin{array}{l}\text { Can you please describe how you decided to } \\
\text { provide technical support to the end-users of the } \\
\text { EHRS? } \\
\text { Have you been provided sufficient budget for this } \\
\text { project? } \\
\text { Can you please describe how FR influence the } \\
\text { EHRS implementation in the Saudi PHCs? } \\
\text { In your opinion, what are the main factors } \\
\text { influenced directly by the FR? } \\
\text { In which way the FR influence these factors? }\end{array}$ \\
\hline $\begin{array}{l}\text { Security and } \\
\text { confidentiality }\end{array}$ & $\begin{array}{l}\text { Can you please describe how data security and } \\
\text { patient's confidentiality were considers? } \\
\text { What steps you take into account to ensure the } \\
\text { systems are secure and patient data are } \\
\text { confidential? }\end{array}$ \\
\hline $\begin{array}{l}\text { Software } \\
\text { selection }\end{array}$ & $\begin{array}{l}\text { Can you please describe how you decided to select } \\
\text { the EHRS? } \\
\text { what are the main criterions you considered while } \\
\text { system selection? } \\
\text { Has the system selected yet? }\end{array}$ \\
\hline $\begin{array}{l}\text { System } \\
\text { usability and } \\
\text { efficiency }\end{array}$ & $\begin{array}{l}\text { In the idea, what would help to ensure the system is } \\
\text { easy to use? and what steps you take into account to } \\
\text { improve the system usability }\end{array}$ \\
\hline
\end{tabular}




\begin{tabular}{|c|c|}
\hline & $\begin{array}{l}\text { What would help to ensure efficiency of the EHRS? } \\
\text { and what steps you take into account to improve the } \\
\text { system efficiency }\end{array}$ \\
\hline $\begin{array}{l}\text { System } \\
\text { interoperability }\end{array}$ & $\begin{array}{l}\text { What steps have you taken into account to ensure } \\
\text { the EHRS will be interoperable with other systems } \\
\text { in the Saudi MOH? }\end{array}$ \\
\hline $\begin{array}{l}\text { User } \\
\text { involvement }\end{array}$ & $\begin{array}{l}\text { Can you please describe how you decided to } \\
\text { involve end-users? } \\
\text { Were adequate time and resources provided to } \\
\text { support end-users involvement? } \\
\text { Were the aim of the project and other } \\
\text { implementation procedures clear to the end-users? } \\
\text { Have you got any feedback from the end-user on } \\
\text { the implemented systems? }\end{array}$ \\
\hline $\begin{array}{l}\text { User } \\
\text { satisfaction }\end{array}$ & $\begin{array}{l}\text { Can you please describe the level of end-user's } \\
\text { satisfaction? } \\
\text { Was there any resistance? } \\
\text { Were there any staffing issues? }\end{array}$ \\
\hline $\begin{array}{l}\text { Barriers and } \\
\text { facilitators }\end{array}$ & $\begin{array}{l}\text { Can you please tell me about the barriers and } \\
\text { challenges during the implementation of the EHRs } \\
\text { in the PHCs in SA? } \\
\text { Were there any difficulties at technical level } \\
\text { What steps you take into account to overcome these } \\
\text { barriers? } \\
\text { Can you please tell me about the facilitators to } \\
\text { implement the EHRs in PHCs in SA? }\end{array}$ \\
\hline Other questions & $\begin{array}{l}\text { Is there any think else that you would like to add to } \\
\text { improve the EHRS implementation? } \\
\text { Do you have any recommendations to enhance the } \\
\text { success of the EHRS for this project and other } \\
\text { projects in the future? } \\
\text { Is there anything you want to tell me at this time? }\end{array}$ \\
\hline
\end{tabular}




\subsubsection{Bias in qualitative research}

Semi-structured and unstructured interviews may face bias issues (Creswell, 2014), because in qualitative research, the reseacher is typically considered to be the instrument of data collection (Creswell, 2014). Bias in interviews can occur as a result of two main issues: researcher performance, and the data collection instruments (interview guide) (Creswell, 2014).

\section{Role of the researcher}

I took care to minimise any potential for bias. Although, as this was my first qualitative research project using an interview approach, I attended some training courses and viewed online media to help me understand what the main issues were likely to be. I then practiced performing interviews with friends and family members to help me learn to moderate and fairly manage the interviews without any bias. I started each interview with an introduction to myself as a $\mathrm{PhD}$ researcher. I tried to make the interviews as friendly as possible. As mentioned earlier in this chapter, I spent sufficient time in the field to familiarise myself with the context and also become familiar with the participants, in particular those who participated in the semi-structured interviews. In addition, all participants were informed of the ethical considerations to ensure their privacy and confidentiality. They all received a copy of the ethical approval (see Appendix A) and consent form. I informed the participants that their data would not be used for any purposes other than this research and that no one would have access to the transcripts except me. In addition, they were told that the data would not be used for evaluation or audit purposes and would not be provided to any one in their organisation. I also stated I would send them a copy of the transcript for validity purposes before they were used. Finally, I explained to them the value of their responses and how they could contribute to my research.

I allowed the participants to choose whether to be interviewed in English or Arabic to avoid any potential for misunderstanding. I also tried to ensure the interview location was quiet, and that the interview could not be heard by others. This was to ensure that the participants could answer questions without fear of being overheard. I took into account that the role of the interviewer was to listen more than speak during the interview. I therefore allowed the participants to talk without any interruptions and avoided making 
comments or asking other questions. I also avoided showing any facial expression or body language that could have influenced the participants answers or change their opinions. As a $\mathrm{PhD}$ researcher with a wide knowledge of EHRS implementation, I tried to keep my knowledge and background to myself to avoid any reflection that may have influenced the data collection during the interviews and the subsequent data interpretation.

I took care not to provide any suggestions or alternative answers to the participants, even if they did not fully answer the question. In addition, I ensured that sufficient time was given to the participants to allow them to adequately answer the question, express their point of view, or describe the process. I avoided confirming participant opinions, even if these opinions were in agreement with my hypotheses. I, therefore, remained neutral at all times. However, the majority of the participants were senior staff at the $\mathrm{MoH}$, so they show more confidence and independence during the interviews.. Moreover, the participants also showed a high level of confidence during the interviews. They believe that giving honest responses to my questions will not result in any harm to them. During the interview with these senior staff members, I experienced some anxiety but tried to remain calm. Moreover, due to cultural nature in SA, as a male researcher I experienced some anxiety during the interviews with female participants. Although I considered myself to be a stranger to the participants, sufficient time spent with the participant prior the interviews allowed me to develop a rapport. I aimed to balance this rapport with keeping an appropriate distance and independence during the interview.

\section{Interview guide related bias}

In regard to the bias related to the interview guide, I took into consideration when asking questions that they would not lead to a specific answer. The aim of this study was to assess PHCs readiness for EHRS implementation, determine barriers and facilitators, and evaluate the impact of $\mathrm{CM}$ and FR, so there were felt to be no sensitive questions or questions that were likely to result in a particular socially desirable response. 


\subsubsection{Qualitative data analysis of semi-structured interviews}

The aim of qualitative data analysis is to "make sense of the collected data" (Guest et al., 2011). The researcher can utilise computer software to collect and analyse qualitative data (Creswell, 2014). NVivo V10 (QSR International) was used to thematically analyse the qualitative data. NVivo helps to manage rich text by categorising it and organising it rather than analysing the text, as other quantitative programs do. Unlike quantitative data analysis software (e.g., SPSS), qualitative data analysis software has less control of the data, it simply assists in organising and categorising the data for better interpretation. Therefore, it is the researcher's role to code and analyse the data. Qualitative data analysis software has fewer functions than quantitative data analysis software.

The qualitative data collected in the semi-structured interviews were analysed using thematic analysis (Bryman, 2012). Thematic analysis is considered to be flexible and accessible and assists the researcher in providing rich and detailed descriptions of the data (Braun \& Clarke, 2006). Thematic analysis is thought to be the foundation of qualitative data analysis (Braun \& Clarke, 2006). According to Braun and Clarke (2006), thematic analysis helps to capture significant information that assists in describing the research question.

I selected thematic analysis because it was considered to be flexible and not restricted to a specific framework or theory. In addition, the decision was made to perform thematic analysis in order to identify patterns in extremely rich information from different perspectives (Bryman, 2012). Thematic analysis was found to be useful to identify patterns and themes that represented implementation procedures. Moreover, thematic analysis enabled the effective categorisation of the main barriers and facilitators and also the procedures of the EHRS implementation plan. Although thematic analysis was found to be very time consuming, NVivo assisted in overcoming this issue. For instance, I was able to recall all highlighted quotes related to one code in just one click instead of searching through all the transcripts again to find the quote.

Once the interviews had finished, I converted the audio file into text format and then a Microsoft Word document. The recordings were transcribed verbatim to make the context of the response and the content of the information clear. Therefore, the process of data 
analysis began immediately once the interviews were transcribed and then translated to English (for all those interviews conducted in Arabic). All transcripts were Microsoft Word files which were imported to the NVivo software. According to Bryman (2012); and Silverman (2013), I performed the thematic analysis in the following six steps:

1. I familiarised myself with the data;

2. Initial codes were generated;

3. The data were searched for themes

4. The themes identified were reviewed

5. The themes were defined and then named

6. A summary of the themes was reported.

Familiarisation started from the transcription process. I also read both Arabic and English transcripts several times to acquire an initial impression of the data in the transcripts and to familiarise myself with the obtained data. In this phase, I also listened to the recorded audio several times to match the transcripts and translation for accuracy purposes. Initial comments about the transcripts were noted. Then I started to label the data and generate the initial codes from the individual transcripts. After an initial reading of the transcripts, I selected the longest and richest transcript to be the first for code extraction. This transcript assisted me in identifying the majority of codes.

I then moved to the next transcript and so on until all transcripts were coded. The number of new codes emerging from the second and subsequent transcripts was fewer than those extracted from the first. The purpose of analysing each transcript, individually, was to detect similar and different opinions (see Table 3.6.2) (Braun \& Clarke, 2006). Moreover, the purpose of data coding was to group similar thoughts and opinions into one term and connect these quotes together within a code. Therefore, searching for codes was based on line by line reading. The quotes in each code varied from one word to a whole paragraph. 
Table 3.6.2:Sample of the tables used to organise quotes for presentation of the final report

\section{Theme One:}

\begin{tabular}{|c|c|c|c|c|}
\hline Codes: & Training & Leadership & Barriers & Communication \\
\hline Interview 1 & $\begin{array}{l}\text { "Training is } \\
\text { a key element } \\
\text { in the } \\
\text { planning." } \\
(D H D 1)\end{array}$ & $\begin{array}{l}\text { "Management } \\
\text { and leadership } \\
\text { affect us } \\
\text { positively and } \\
\text { they have a very } \\
\text { significant role } \\
\text { in contributing to } \\
\text { facilitating our } \\
\text { mission." (SD 1) }\end{array}$ & $\begin{array}{l}\text { "Connectivity } \\
\text { is considered } \\
\text { one of the main } \\
\text { barriers." } \\
\text { (GM1) }\end{array}$ & $\begin{array}{l}\quad \text { "We } \\
\text { hold continuous } \\
\text { workshops." } \\
(\text { GM 2) }\end{array}$ \\
\hline Interview 2 & & & $\begin{array}{l}\text { "Of the main } \\
\text { obstacles is } \\
\text { training." } \\
\text { (Analyst 1) }\end{array}$ & \\
\hline Interview 3 & $\begin{array}{l}\text { "The training } \\
\text { courses } \\
\text { received } \\
\text { much } \\
\text { attention in } \\
\text { the } \\
\text { planning." } \\
\text { (HD 1) }\end{array}$ & & $\begin{array}{l}\text { "One of } \\
\text { biggest } \\
\text { challenges is } \\
\text { finding talented } \\
\text { people in } \\
\text { specific areas, } \\
\text { especially in } \\
\text { SA." (GM1) }\end{array}$ & $\begin{array}{l}\text { "Committees } \\
\text { have been } \\
\text { formed to } \\
\text { communicate } \\
\text { with other } \\
\text { project team } \\
\text { members." (HD } \\
\text { 3) }\end{array}$ \\
\hline Interview 4 & $\begin{array}{l}\text { "Training } \\
\text { courses will } \\
\text { be presented } \\
\text { in both } \\
\text { Arabic and } \\
\text { English } \\
\text { languages." } \\
\text { (DHD1) }\end{array}$ & $\begin{array}{l}\text { "Support from } \\
\text { senior } \\
\text { management is } \\
\text { one of the most } \\
\text { important } \\
\text { facilitators of } \\
\text { successful EHRS } \\
\text { implementation." } \\
\text { (HD 1) }\end{array}$ & $\begin{array}{l}\text { "Geographical } \\
\text { nature is a } \\
\text { challenge for } \\
\text { the MoH." } \\
\text { (HD2) }\end{array}$ & $\begin{array}{l}\text { "We hold } \\
\text { continuous } \\
\text { workshops." } \\
\text { (GM 2) }\end{array}$ \\
\hline Interview 5 & & & $\begin{array}{l}\text { "The obstacles } \\
\text { are too many." } \\
\text { (SD1) }\end{array}$ & \\
\hline
\end{tabular}


Once all transcripts were labelled and coded, I searched the data set to determine patterns (themes). I read all emergent codes and sub-codes to determine the broader patterns. The identified patterns were treated as a potential theme. Therefore, the themes generated by thematic analysis are driven by the codes generated from the transcripts (Bryman, 2012). Initially, the analysis resulted in twelve categories (potential themes). The theme formulation process was carried out by drawing a mind-map, which included all the generated codes and sub-codes. Subsequently, similar codes were combined and then labelled using best themes that match the similar codes. The number of identified categories fell from twelve to six, through the aggregation of similar categories. The identified themes, presented in the results, were felt to be the most appropriate to represent the emergent codes from all transcripts. All generated codes without exception were categorised into the identified themes and then included in the final report. The next step in the thematic analysis was to review the identified themes. In addition to the previous step, I reread the transcripts and reviewed the codes and sub-codes to ensure that they covered the required information in the transcripts and that the data available were appropriate to address the study objectives.

The final stage of thematic analysis was to report the analysed data. The presentation of the final report was based on the themes, sub-themes, codes and sub-codes. The final report includes six main themes, each with several codes and sub-codes. Each code and sub-codes include several quotes. However, the number of quotes varied from one code to another. Some codes comprised multiple quotes, while others comprised only one. Multiple quotes were sometimes in agreement, and sometimes in disagreement.

\subsubsection{Validity and Reliability}

Although validity and reliability have the same concept in both qualitative and quantitative methods, the terms used in qualitative research are different than those used in quantitative research (see Table 3.6.3) (Bryman, 2012). For instance, internal validity in quantitative research means credibility in qualitative research. Validity in qualitative research starts from early data collection procedures (e.g., sampling technique) (Tongco, 2007). Therefore, I conducted several procedures in order to ensure the validity of this 
study. In preparing for this study I spent approximately four months at the headquarters of the Saudi MoH. In that time, I submitted the required documents to obtain ethical approval from the relevant departments at the $\mathrm{MoH}$, and I conducted the first quantitative study in the same place (see Section 3.3). During this period, I tried to familiarise myself with the context, phenomenon and the study participants and their role in the project to improve decision-making regarding the selection of the participants. As previously mentioned, all participants were purposively selected. However, there were very limited subjects available to me which may affect the validity.

Table 3.6.3:Validity and reliability terms used in quantitative and qualitative research

\begin{tabular}{|c|c|}
\hline Quantitative & Qualitative \\
\hline Internal validity & Credibility \\
\hline External validity & Transferability \\
\hline Reliability & Dependability \\
\hline Objectivity & Confirmability \\
\hline
\end{tabular}

\section{$\underline{\text { Pilot interview }}$}

To improve validity, the first interview was conducted with the Deputy Head of Department (DHD1) as a pilot to determine the expected length of the interviews. In addition, the pilot study was conducted to test the interview guide and ensure that it could produce rich data necessary to achieve the study objectives. As a result, a few additional questions were included in the interview guide and the suggested time allocated for the interviews was amended accordingly. On the other hand, no new information emerged from the last two interviews. Therefore, I terminated data collection at interview number eleven. It has been recommended that interviews should be terminated when no new information emerges (Silverman, 2013).

\section{Transcription and translation checking}

Once all interviews conducted and transcribed, I removed the data that would identify any individuals and then the transcribed data were compared against the original audio file to ensure accuracy. Thereafter, I sent the Arabic transcripts to an official translation 
agent in SA, to ensure the translated data were correct. In addition, both Arabic and English copies of the transcripts were given to three $\mathrm{PhD}$ students at Swansea University for reviewing and proofreading. These PhD students are native speakers of the Arabic language and their research is based on English literature and language translation. They provided good feedback about the translated transcripts.

\section{$\underline{\text { Involvement of inter-rater }}$}

Thereafter, all transcripts were sent to another independent researcher who has experience in thematic analysis for other observations (Bryman, 2012). The inter-rater conducted the above six stages of thematic analysis independently. Although, there was about eighty percent agreement regarding most of the themes and codes generated, there was disagreement about some of the codes and their quotes. English is my second language and also that of the independent inter-rater. As a result, at times, different terms were used for similar codes. According to Bryman (2012), minor differences between multiple reports when involving an inter-rater are acceptable, since qualitative research generates a large data set.

\section{Member checking}

For credibility purposes, I conducted member-checking (Bryman, 2012). The transcript and final report of the data with the field notes, codes and themes were sent to the participants for verification. Although only three participants replied to me, minor comments and further clarifications, which include additional information, were received from four participants. The returned comments did not conflict with any of the responses from the interviews. Most of the comments were about dates and numbers, such as the number of PHCs in SA and the number of PHCs that had implemented EHRS. Moreover, one of the interviewees asked to remove the name of a commercial company mentioned during the interview. All participants will receive a copy of this thesis, as I promised.

\section{Audit and documentation}

Every step of data collection and analysis, including those mentioned, above has been documented and also reviewed by a supervision team, whether by email, on campus 
meetings, or Skype meetings (see Figure 3.6.1). Figure 3.6.1 is an example of how all procedures in this study were documented to improve validity. Documents such as transcripts, audio recordings, the interview guide, field notes, consent forms and NVivo files were saved and also shared with my supervisors. The supervision team audit included a revision of transcripts and other documents, data collection monitoring and guidance, and proofreading of the final report. In addition, the final report which included all codes and themes and quotes was examined by the supervisory team to ensure the accuracy of the findings. 


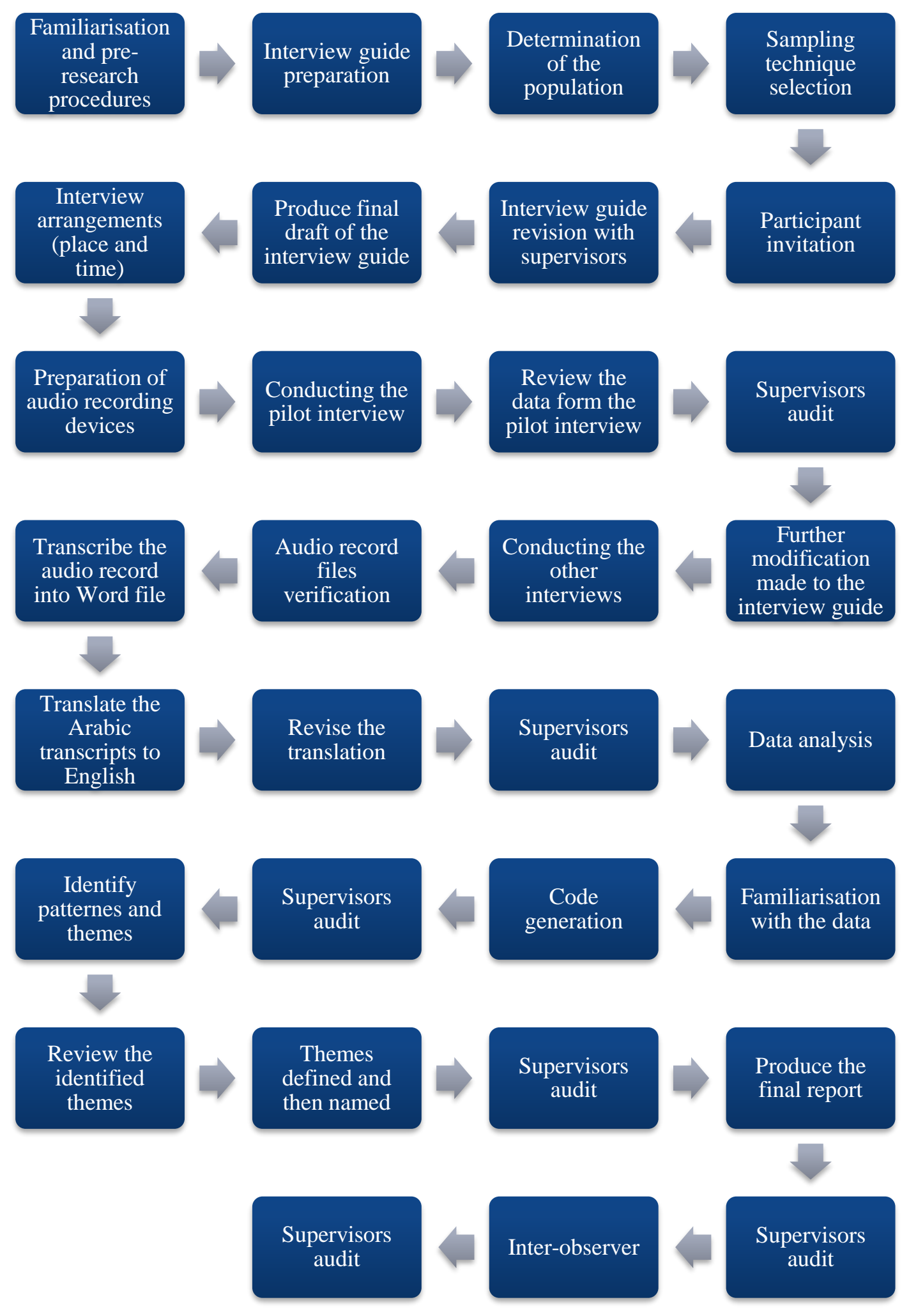

Figure 3.6.1: Semi-structured interviews journey. 


\subsection{Chapter summary}

This chapter summaries the four studies conducted to achieve the main aim of this thesis and obtain a broad view of EHRS implementation in PHCs in SA. The conducted studies were directed to three different populations; two studies were directed to the project team at the Saudi MoH, one to EHRS end-users in PHCs, and the final to potential users of the EHRS at PHCs. Moreover, to achieve the main aim of this thesis, a mixed-methods approach was utilised: three quantitative studies, and one qualitative study. While quantitative data were analysed using SPSS, qualitative data were analysed using NVivo. Both descriptive and inferential, non-parametric tests were performed to analyse the quantitative data. On the other hand, thematic analysis methods were adopted to analyse the qualitative data. The next chapter presents the findings from Study One and aims to assess PHC readiness for EHRS implementation from a project team perspective. 
4 Chapter Four: Study One: Assessing PHC Readiness for EHRS Implementation from the Project Team Perspective 


\subsection{Introduction}

This chapter reports on statistics of the results obtained from the questionnaire that was distributed to thirty-one participants. The aim of the questionnaire was to assess Saudi PHCs readiness for the implementation of a new EHRS from the project team perspective, and also to evaluate the impact of Financial Recourses (FR) and Centralised Management (CM). To achieve the above, descriptive statistics were utilised. This test displays all obtained data and then predicts the level of PHCs readiness to the implementation of the EHRS with accordance to each one of the examined eight factors (see Chapter Three, Section 3.3). In addition, descriptive statistics will help to determine which factor is most influenced by FR and CM.

\subsection{Scale reliability:}

This section will look at the consistency of the answers within each of the scales (themes); this is often referred to as internal reliability of the scales. It is essential that such scales are highly reliable to ensure that all items in each of the scales are measuring the same factor. Reliability in this context is measured through a Cronbach's Alpha test which measures consistency in terms of percentages ranging from 0 to $100 \%(0-1)$. Reliability scores above seventy percent are often considered acceptable (Field, 2013). Table 4.2.1 reveals the reliability score for each of the ten scales in the questionnaire. As seen in Table 4.2.1, all scales in this study were acceptable and had a good reliability score. 
Table 4.2.1: Cronbach's Alpha reliability measure and the numbers of main scale and sub-scale items

\begin{tabular}{|l|c|c|}
\hline Scale & N & $\begin{array}{c}\text { Cronbach's } \\
\text { Alpha }\end{array}$ \\
\hline Readiness & $\mathbf{4 8}$ & $\mathbf{0 . 9 5}$ \\
\hline Resources & 6 & 0.79 \\
\hline End-user & 6 & 0.82 \\
\hline Technology & 6 & 0.75 \\
\hline Knowledge & 6 & 0.70 \\
\hline Processes & 6 & 0.94 \\
\hline $\begin{array}{l}\text { Values and Goals } \\
\text { Management }\end{array}$ & 6 & 0.87 \\
\hline $\begin{array}{l}\text { Structure } \\
\text { Administrative }\end{array}$ & 6 & 0.90 \\
\hline Support & 6 & 0.83 \\
\hline Impact of CM & $\mathbf{9}$ & $\mathbf{0 . 9 4}$ \\
\hline $\begin{array}{l}\text { Impact of F } \\
\text { Entire } \\
\text { Questionnaire }\end{array}$ & $\mathbf{1 1}$ & $\mathbf{0 . 9 3}$ \\
\hline
\end{tabular}

\subsection{Descriptive statistics of participant responses}

A total of fifty-three individuals met the selection criteria with the association of the sampling strategy used. Out of the fifty-three, only thirty-one participated and completed the questionnaire, indicating a response rate of 59\%. Table 4.3.1 shows the percentage of male and female participation in the questionnaire. It is evident that participation was male dominant, with $80.6 \%$ of participants being male. Female participation was found to be only $16.1 \%$. This reflects the actual proportion of females and males in the Saudi $\mathrm{MoH}$, where the majority of the staff are male, particularly the targeted population of this study. Out of thirty-one participants, the gender of one person was not recorded.

Table 4.3.1: Participant distribution based on gender

\begin{tabular}{|c|c|c|}
\hline Gender & Frequency & Percent \\
\hline Male & 25 & 80.6 \\
\hline Female & 5 & 16.1 \\
\hline Total & 30 & 96.8 \\
\hline
\end{tabular}


Table 4.3.2 shows information about participants who had previous involvement with an EHRS implementation. The table indicates that among the thirty-one participants, eighteen $(58.1 \%)$ had been involved in previous EHRS implementation, and thirteen $(41.9 \%)$ had never been involved in any EHRS implementation.

Table 4.3.2:Participant distribution based on previous involvement in EHRS implementation projects

\begin{tabular}{|l|c|c|}
\hline Involvement & Frequency & Percent \\
\hline YES & 18 & 58.1 \\
\hline NO & 13 & 41.9 \\
\hline Total & 31 & 100.0 \\
\hline
\end{tabular}

Table 4.3.3 shows the participants' role in the Saudi $\mathrm{MoH}$, where the survey was conducted. The participants in the survey were found to be from diverse professional roles. The assistants formed the highest number of participants, at twenty-one (67.7\%). Only one deputy manager participated in this study, and three participants from other positions.

Table 4.3.3: Participant distribution based on position

\begin{tabular}{|l|c|c|}
\hline Position & Frequency & Percent \\
\hline General Manager & 3 & 9.7 \\
\hline Deputy Manager & 1 & 3.2 \\
\hline Head of Department & 3 & 9.7 \\
\hline Deputy Head of Department & 3 & 9.7 \\
\hline Assistant & 21 & 67.7 \\
\hline Total & 31 & 100.0 \\
\hline
\end{tabular}

Table 4.3.4 shows the nature of the role played by the participants during EHRS implementation. This can be either direct involvement or cooperation with the process at various stages through indirect involvement. Out of thirty-one participants, twenty $(64.5 \%)$ declared that they were directly involved in the process of implementation and five $(16.1 \%)$ declared that they aided the process through an indirect connection. Six (19.4\%) participants did not declare the nature of their involvement. 
Table 4.3.4: Participant distribution based on role in the implementation project

\begin{tabular}{|l|c|c|}
\hline Role & Frequency & Percent \\
\hline Direct & 20 & 64.5 \\
\hline Indirect & 5 & 16.1 \\
\hline Total & 25 & 80.6 \\
\hline
\end{tabular}

\subsubsection{Organisational Information Technology/Systems Innovation Readiness Scale (OITIRS)}

This part of the analysis examined PHCs readiness for the implementation of an EHRS. Readiness was examined through eight sub-themes/categories; namely resources, enduser, technology, knowledge, processes, values and goals, management structure and administrative support. Each of the following sections will target one of these categories. The findings, in all tables in this section, aim to measure PHC readiness for EHRS implementation. The items, in all tables in this section, are ranked based on total agreement. Total agreement was calculated, based on the sum of percentages of three categories: "somewhat agree, agree, and strongly agree" (see Chapter Three).

\section{PHCs readiness at the resources level}

The findings in Table 4.3.5 show a high level of PHC readiness to implement the EHRS at the resources level, where all six items were provided in this scale had a median score between five and seven. It can be seen from Table 4.3.5 that Item 1), "Funding is adequate for completion of EHRS implementation", ranked first among all items with a median of seven and total agreement (93.6\%). This was followed by 2) "The project budget includes training/retraining costs", which generated the same median and slightly less agreement $(83.6 \%)$. The last item ranked sixth in the resources table, "Good quality vendor support for the EHRS is typically available", with a median score of five (64.6\% agreement). Overall it can be concluded that all items had a median score above the no opinion point of four, which shows a high level of agreement with all items at the readiness at resources level. 
Table 4.3.5: Participant responses to items representing PHC readiness for EHRS implementation at resources level

\begin{tabular}{|c|c|c|c|c|c|c|c|c|c|c|c|}
\hline \multicolumn{2}{|l|}{$\underset{\overparen{\Xi}}{\widetilde{\Xi}}$} & 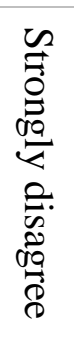 & 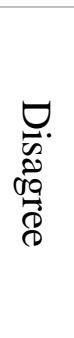 & 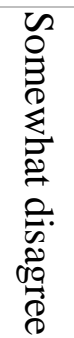 & $\begin{array}{l}Z \\
\text { Z } \\
0 \\
o \\
0 . \\
\text { E. }\end{array}$ & 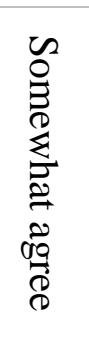 & 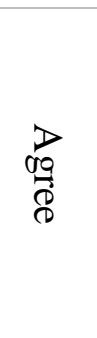 & 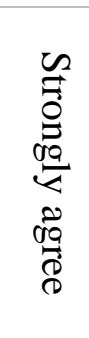 & 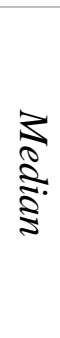 & 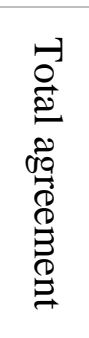 & 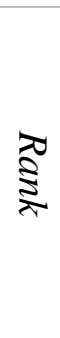 \\
\hline \multirow{2}{*}{$\begin{array}{l}\text { Funding is } \\
\text { adequate for } \\
\text { completion of } \\
\text { EHRS } \\
\text { implementation }\end{array}$} & $\mathrm{N}$ & & & & 2 & & 6 & 23 & \multirow[b]{2}{*}{7} & 29 & \multirow[b]{2}{*}{1} \\
\hline & $\%$ & & & & 6.5 & & 19.4 & 74.2 & & 93.6 & \\
\hline \multirow{2}{*}{$\begin{array}{l}\text { The project budget } \\
\text { includes } \\
\text { training/retraining } \\
\text { costs }\end{array}$} & $\mathrm{N}$ & & 1 & & 4 & 3 & 5 & 17 & \multirow[b]{2}{*}{7} & 25 & \multirow[b]{2}{*}{2} \\
\hline & $\%$ & & 3.2 & & 12.9 & 9.7 & 16.1 & 54.8 & & 83.6 & \\
\hline \multirow{2}{*}{$\begin{array}{l}\text { There is a good } \\
\text { ratio of full-time } \\
\text { in-house to } \\
\text { contract IT staff to } \\
\text { support the project }\end{array}$} & $\mathrm{N}$ & 2 & & & 4 & 8 & 11 & 6 & \multirow[b]{2}{*}{6} & 25 & \multirow[b]{2}{*}{3} \\
\hline & $\%$ & 6.5 & & & 12.9 & 25.8 & 35.5 & 19.4 & & 80.6 & \\
\hline \multirow{2}{*}{$\begin{array}{l}\text { The project budget } \\
\text { is consistent with } \\
\text { the organisation's } \\
\text { strategic plan }\end{array}$} & $\mathrm{N}$ & 1 & 1 & 1 & 4 & 3 & 9 & 12 & \multirow[b]{2}{*}{6} & 24 & \multirow[b]{2}{*}{4} \\
\hline & $\%$ & 3.2 & 3.2 & 3.2 & 12.9 & 9.7 & 29.0 & 38.7 & & 77.4 & \\
\hline \multirow{2}{*}{$\begin{array}{l}\text { Project teams have } \\
\text { included both } \\
\text { technical support } \\
\text { staff and users }\end{array}$} & $\mathrm{N}$ & 1 & 1 & 2 & 5 & 2 & 13 & 7 & \multirow[b]{2}{*}{6} & 22 & \multirow[b]{2}{*}{5} \\
\hline & $\%$ & 3.2 & 3.2 & 6.5 & 16.1 & 6.5 & 41.9 & 22.6 & & 71.0 & \\
\hline \multirow{2}{*}{$\begin{array}{l}\text { Good quality } \\
\text { vendor support for } \\
\text { the EHRS is } \\
\text { typically available }\end{array}$} & $\mathrm{N}$ & 2 & 2 & 2 & 5 & 8 & 10 & 2 & \multirow[b]{2}{*}{5} & 20 & \multirow[b]{2}{*}{6} \\
\hline & $\%$ & 6.5 & 6.5 & 6.5 & 16.1 & 25.8 & 32.3 & 6.5 & & 64.6 & \\
\hline
\end{tabular}

\section{PHC readiness at the end-user level}

As can be seen in Table 4.3.6, the end-user scale included six items enquiring about the level of PHC readiness to implement EHRS at the end-user level. Descriptive statistics Item 1) "Adequate training is available to support users" ranked first among all items in this scale, where the median was found to be six. This indicates that, according to the participants, adequate training is indeed available to users (77.5\% agreement). This item was followed by 2) "A core group of users (champions) is available to support implementation", which also shows the availability of support in the form of a core group 
of users (Median=6 and $77.5 \%$ agreement). The least ranked item was 6) "User competencies are appropriately incorporated into job performance criteria" (Median=5 and $61.4 \%$ agreement). Again, and similar to the previous scale, all items achieved a median above the neutral point of four, which indicates that the number of individuals agreeing with the statements was greater than those disagreeing. However, the level of end-user readiness was found to be lower than resource readiness.

Table 4.3.6: Participant responses to items representing PHC readiness for EHRS implementation at the end-user level.

\begin{tabular}{|c|c|c|c|c|c|c|c|c|c|c|c|}
\hline \multicolumn{2}{|l|}{$\underset{\mathbb{\Xi}}{\widetilde{\Xi}}$} & 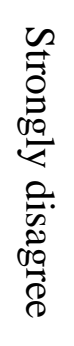 & 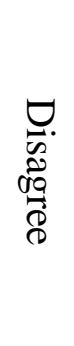 & 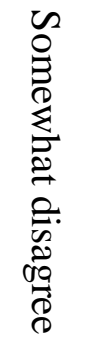 & $\begin{array}{l}z \\
o \\
o \\
o \\
\\
\vdots \\
o\end{array}$ & 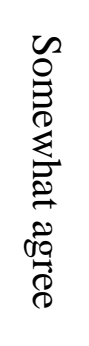 & $\underset{\substack{10 \\
8}}{8}$ & 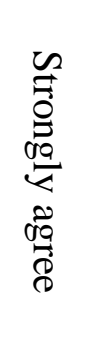 & $\begin{array}{c}\vdots \\
\vdots \\
\vdots \\
\vdots \\
\vdots \\
\vdots\end{array}$ & 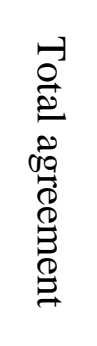 & 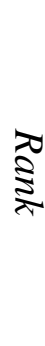 \\
\hline \multirow{2}{*}{$\begin{array}{l}\text { Adequate training } \\
\text { is available to } \\
\text { support users }\end{array}$} & $\mathrm{N}$ & 1 & 2 & & 4 & 2 & 7 & 15 & \multirow[b]{2}{*}{6} & 24 & \multirow[b]{2}{*}{1} \\
\hline & $\%$ & 3.2 & 6.5 & & 12.9 & 6.5 & 22.6 & 48.4 & & 77.5 & \\
\hline \multirow{2}{*}{$\begin{array}{l}\text { A core group of } \\
\text { users (champions) } \\
\text { is available to } \\
\text { support } \\
\text { implementation }\end{array}$} & $\mathrm{N}$ & 2 & 1 & 2 & 2 & 3 & 9 & 12 & \multirow[b]{2}{*}{6} & 24 & \multirow[b]{2}{*}{2} \\
\hline & $\%$ & 6.5 & 3.2 & 6.5 & 6.5 & 9.7 & 29.0 & 38.8 & & 77.5 & \\
\hline Users are & $\mathrm{N}$ & 2 & 3 & 1 & 2 & 5 & 14 & 4 & \multirow[b]{2}{*}{6} & 23 & \multirow[b]{2}{*}{3} \\
\hline $\begin{array}{l}\text { implementation } \\
\text { im EHRS }\end{array}$ & $\%$ & 6.5 & 9.7 & 3.2 & 6.5 & 16.1 & 45.2 & 12.9 & & 74.2 & \\
\hline \multirow{2}{*}{$\begin{array}{l}\text { Most users have } \\
\text { an adequate level } \\
\text { of computer } \\
\text { literacy }\end{array}$} & $\mathrm{N}$ & & 1 & 4 & 4 & 7 & 12 & 3 & \multirow[b]{2}{*}{5} & 22 & \multirow[b]{2}{*}{4} \\
\hline & $\%$ & & 3.2 & 12.9 & 12.9 & 22.6 & 38.7 & 9.7 & & 71.0 & \\
\hline \multirow{2}{*}{$\begin{array}{l}\text { Users are } \\
\text { typically } \\
\text { supportive of an } \\
\text { EHRS }\end{array}$} & $\mathrm{N}$ & & 2 & 1 & 6 & 9 & 9 & 3 & \multirow[b]{2}{*}{5} & 21 & \multirow[b]{2}{*}{5} \\
\hline & $\%$ & & 6.5 & 3.2 & 19.4 & 29.0 & 29.0 & 9.7 & & 70.0 & \\
\hline \multirow{2}{*}{$\begin{array}{l}\text { User } \\
\text { competencies are } \\
\text { appropriately } \\
\text { incorporated into } \\
\text { job performance } \\
\text { criteria }\end{array}$} & $\mathrm{N}$ & 2 & 1 & 2 & 7 & 11 & 6 & 2 & \multirow[b]{2}{*}{5} & 19 & \multirow[b]{2}{*}{6} \\
\hline & $\%$ & 6.5 & 3.2 & 6.5 & 22.6 & 35.5 & 19.4 & 6.5 & & 61.4 & \\
\hline
\end{tabular}




\section{PHC readiness at the technology level}

PHC readiness, for the implementation of the EHRS at the technology level, was investigated through six items. Table 4.3.7, below, shows that there is more agreement across all items compared to disagreement, and all items had a median score between five and six, showing a high level of agreement for all items. 1) "Research and development activities to learn about new technology are supported" ranked first, in the technological scale, with a median score of six and agreement of $80.7 \%$. This was followed by 2) "Development of information systems is based on current market trends" (median of six and agreement of 74.2\%). The least agreement score was generated for 6) "EHRS project implementation time frames are usually adequate", which had the lowest median (five) and the lowest agreement (61.3\%). 
Table 4.3.7: Participant responses to items representing PHC readiness for EHRS implementation at the technology level.

\begin{tabular}{|c|c|c|c|c|c|c|c|c|c|c|c|}
\hline \multicolumn{2}{|l|}{ 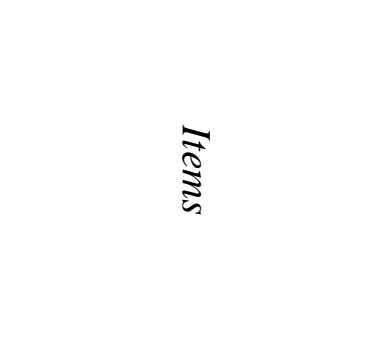 } & 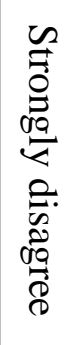 & 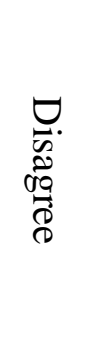 & 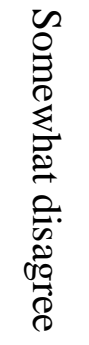 & 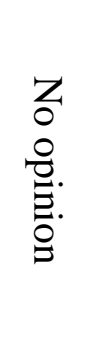 & 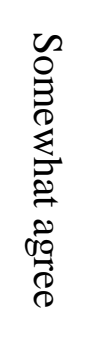 & 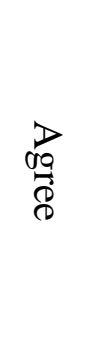 & 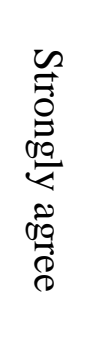 & 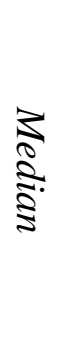 & 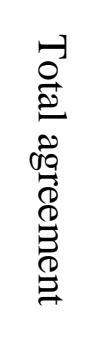 & 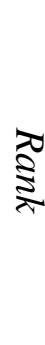 \\
\hline \multirow{2}{*}{$\begin{array}{l}\text { Research and } \\
\text { development } \\
\text { activities to learn } \\
\text { about new } \\
\text { technology are } \\
\text { supported }\end{array}$} & $\mathrm{N}$ & 2 & 1 & & 3 & 7 & 16 & 2 & \multirow[b]{2}{*}{6} & 25 & \multirow[b]{2}{*}{1} \\
\hline & $\%$ & 6.5 & 3.2 & & 9.7 & 22.6 & 51.6 & 6.5 & & 80.7 & \\
\hline \multirow{2}{*}{$\begin{array}{l}\text { Development of } \\
\text { information } \\
\text { systems is based } \\
\text { on current } \\
\text { market trends }\end{array}$} & $\mathrm{N}$ & 1 & 3 & & 4 & 7 & 13 & 3 & \multirow[b]{2}{*}{6} & 23 & \multirow[b]{2}{*}{2} \\
\hline & $\%$ & 3.2 & 9.7 & & 12.9 & 22.6 & 41.9 & 9.7 & & 74.2 & \\
\hline \multirow{2}{*}{$\begin{array}{l}\text { There is a good } \\
\text { fit between } \\
\text { organisational } \\
\text { and EHRS } \\
\text { implementation } \\
\text { strategic plans }\end{array}$} & $\mathrm{N}$ & 2 & 1 & 3 & 3 & 4 & 3 & 15 & \multirow[b]{2}{*}{6} & 22 & \multirow[b]{2}{*}{3} \\
\hline & $\%$ & 6.5 & 3.2 & 9.7 & 9.7 & 12.9 & 9.7 & 48.4 & & 71.0 & \\
\hline \multirow{2}{*}{$\begin{array}{l}\text { There are good } \\
\text { quality vendor } \\
\text { contracts }\end{array}$} & $\mathrm{N}$ & 1 & 2 & 1 & 6 & 4 & 3 & 14 & \multirow{2}{*}{6} & 21 & \multirow{2}{*}{4} \\
\hline & $\%$ & 3.2 & 6.5 & 3.2 & 19.4 & 12.9 & 9.7 & 45.2 & & 67.8 & \\
\hline \multirow{2}{*}{$\begin{array}{l}\text { Current work } \\
\text { practices are } \\
\text { adequately } \\
\text { supported by } \\
\text { existing } \\
\text { information } \\
\text { systems }\end{array}$} & $\mathrm{N}$ & & 6 & 2 & 2 & 4 & 16 & 1 & \multirow[b]{2}{*}{6} & 21 & \\
\hline & $\%$ & & 19.4 & 6.5 & 6.5 & 12.9 & 51.6 & 3.2 & & 67.7 & 5 \\
\hline \multirow{2}{*}{$\begin{array}{l}\text { EHRS project } \\
\text { implementation } \\
\text { time frames are } \\
\text { usually adequate }\end{array}$} & $\mathrm{N}$ & & 2 & 6 & 4 & 5 & 12 & 2 & \multirow[b]{2}{*}{5} & 19 & \multirow[b]{2}{*}{6} \\
\hline & $\%$ & & 6.5 & 19.4 & 12.9 & 16.1 & 38.7 & 6.5 & & 61.3 & \\
\hline
\end{tabular}

\section{PHC readiness at the knowledge level}

Table 4.3.8 below illustrates all items for PHC readiness at the knowledge level. There was a higher proportion of agreement compared to disagreement, which was evident through the median and agreement scores. In terms of ranking, Item 1) "There is a lot of 
knowledge about the on-going development needs of EHRS support staff', was the highest ranked, with a median of six and an agreement of $83.9 \%$. This was followed by 2) "Knowledge about how EHRS implementation is being used by other organisations is available", with a median of six and an agreement of $80.7 \%$. The least ranked item was "In the past, EHRS users have been included in decision-making processes". This item showed a median of four and a total agreement of $48.4 \%$. Whereas, the proportion of people responding in a neutral or disagreement is greater than those responding positively. However, the majority of the items in this scale had a median score between five and six, which is above the neutral point of four. This shows a high level of agreement with most items. Hence, corresponding to the objective of this study, Table 4.3.8 reveals a good level of PHC readiness for EHRS implementation in terms of knowledge. 
Table 4.3.8: Participant responses to items representing $P H C$ readiness for EHRS implementation at the knowledge level

\begin{tabular}{|c|c|c|c|c|c|c|c|c|c|c|c|}
\hline \multicolumn{2}{|l|}{$\begin{array}{l}\widetilde{\Xi} \\
\stackrel{\Xi}{5}\end{array}$} & 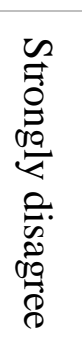 & 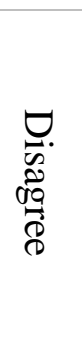 & 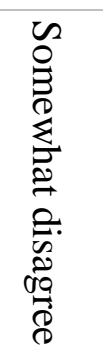 & $\begin{array}{l}Z \\
\text { z } \\
\circ \\
\text { o्. } \\
\text { E. } \\
0\end{array}$ & 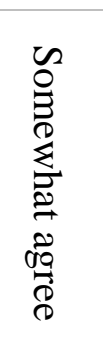 & $\underset{D}{\frac{D}{80}}$ & 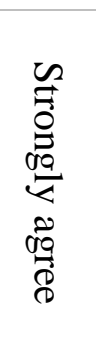 & $\begin{array}{l}\frac{1}{0} \\
\stackrel{2}{\vdots} \\
\vdots\end{array}$ & 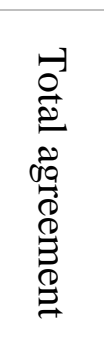 & 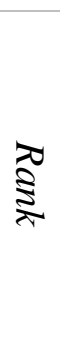 \\
\hline \multirow{2}{*}{$\begin{array}{l}\text { There is a lot of } \\
\text { knowledge about } \\
\text { the on-going } \\
\text { development } \\
\text { needs of EHRS } \\
\text { support staff }\end{array}$} & $\mathrm{N}$ & 1 & 2 & 2 & & 6 & 17 & 3 & & 26 & \\
\hline & $\%$ & 3.2 & 6.5 & 6.5 & & 19.4 & 54.8 & 9.7 & 6 & 83.9 & 1 \\
\hline \multirow[b]{2}{*}{$\begin{array}{l}\text { Knowledge is } \\
\text { available about } \\
\text { how EHRS } \\
\text { implementation is } \\
\text { being used by } \\
\text { other } \\
\text { organisations }\end{array}$} & $\mathrm{N}$ & 2 & & 1 & 3 & 6 & 4 & 15 & & 25 & \\
\hline & $\%$ & 6.5 & & 3.2 & 9.7 & 19.4 & 12.9 & 48.4 & 6 & 80.7 & 2 \\
\hline \multirow{2}{*}{$\begin{array}{l}\text { There is a lot of } \\
\text { knowledge about } \\
\text { EHRS } \\
\text { operational and } \\
\text { capital budget } \\
\text { trends }\end{array}$} & $\mathrm{N}$ & 3 & & & 4 & 15 & 6 & 3 & & 24 & \\
\hline & $\%$ & 9.7 & & & 12.9 & 48.4 & 19.4 & 9.7 & 5 & 77.5 & 3 \\
\hline \multirow{2}{*}{$\begin{array}{l}\text { Historically, the } \\
\text { strategic and } \\
\text { EHRS } \\
\text { implementation } \\
\text { goals have been } \\
\text { integrated }\end{array}$} & $\mathrm{N}$ & 1 & 1 & & 6 & 1 & 15 & 7 & & 23 & \\
\hline & $\%$ & 3.2 & 3.2 & & 19.4 & 3.2 & 48.4 & 22.6 & 6 & 74.2 & 4 \\
\hline \multirow[b]{2}{*}{$\begin{array}{l}\text { Administrators } \\
\text { are very } \\
\text { knowledgeable } \\
\text { about EHRS } \\
\text { based on their } \\
\text { past experience }\end{array}$} & $\mathrm{N}$ & 2 & & 7 & 6 & 4 & 8 & 4 & & 16 & \\
\hline & $\%$ & 6.5 & & 22.6 & 19.4 & 12.9 & 25.8 & 12.9 & 5 & 51.6 & 5 \\
\hline \multirow{2}{*}{$\begin{array}{l}\text { In the past, EHRS } \\
\text { users have been } \\
\text { included in } \\
\text { decision-making } \\
\text { processes }\end{array}$} & $\mathrm{N}$ & 3 & 2 & 5 & 6 & 3 & 9 & 3 & & 15 & \\
\hline & $\%$ & 9.7 & 6.5 & 16.1 & 19.4 & 9.7 & 29.0 & 9.7 & 4 & 48.4 & 6 \\
\hline
\end{tabular}




\section{PHCs readiness at the process level}

PHC readiness for EHRS implementation at the process level was examined through six items (see Table 4.3.9), all of which generated similar total agreement, as the proportion of people responding in a neutral or agreement was greater than those responding negatively. The highest ranked item was 1) "EHRS implementation needs are routinely incorporated into the organisation's business processes", with an agreement of $74.1 \%$, while Item 6) "EHRS implementation decision-makers are adequately represented on key organisational committees", ranked last, generating a total agreement of $67.7 \%$. Overall, the below scale show very high level of agreement toward the PHCs readiness for EHRS implementation at the process level. 
Table 4.3.9: Participant responses to items representing PHC readiness for EHRS implementation at the process level

\begin{tabular}{|c|c|c|c|c|c|c|c|c|c|c|c|}
\hline \multicolumn{2}{|l|}{ 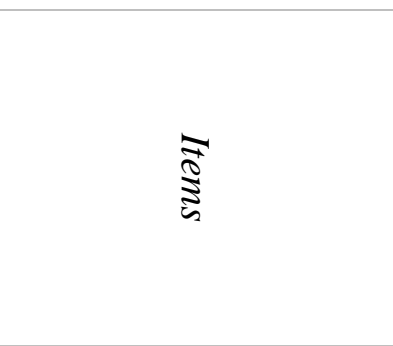 } & 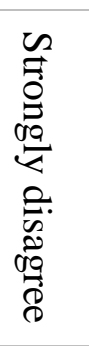 & 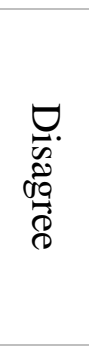 & 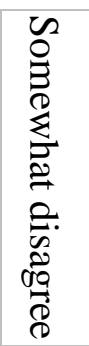 & $\begin{array}{l}Z \\
\text { z } \\
o \\
\text { o. } \\
\text { E. } \\
0\end{array}$ & 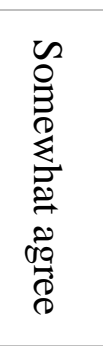 & $\underset{D}{\stackrel{D}{0}}$ & 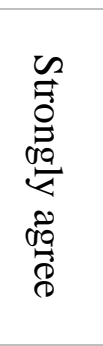 & 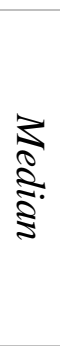 & 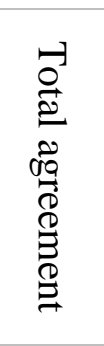 & 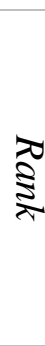 \\
\hline \multirow[b]{2}{*}{$\begin{array}{l}\text { EHRS } \\
\text { implementation } \\
\text { needs are } \\
\text { routinely } \\
\text { incorporated into } \\
\text { the organisation's } \\
\text { business processes }\end{array}$} & $\mathrm{N}$ & 5 & & & 3 & 1 & 5 & 17 & & 23 & \\
\hline & $\%$ & 16.1 & & & 9.7 & 3.2 & 16.1 & 54.8 & 7 & 74.1 & 1 \\
\hline \multirow[b]{2}{*}{$\begin{array}{l}\text { The most } \\
\text { appropriate } \\
\text { individuals are } \\
\text { involved in the } \\
\text { development of } \\
\text { the EHRS } \\
\text { implementation } \\
\text { strategic plan }\end{array}$} & $\mathrm{N}$ & 1 & 4 & & 3 & 3 & 5 & 15 & & 23 & \\
\hline & $\%$ & 3.2 & 12.9 & & 9.7 & 9.7 & 16.1 & 48.4 & 6 & 74.2 & 2 \\
\hline \multirow[b]{2}{*}{$\begin{array}{l}\text { Adequate } \\
\text { communication } \\
\text { mechanisms exist } \\
\text { to support shared } \\
\text { communication } \\
\text { across all } \\
\text { organisational } \\
\text { levels }\end{array}$} & $\mathrm{N}$ & & 2 & 3 & 3 & 2 & 13 & 8 & & 23 & \\
\hline & $\%$ & & 6.5 & 9.7 & 9.7 & 6.5 & 41.9 & 25.8 & 6 & 74.2 & 3 \\
\hline \multirow{2}{*}{$\begin{array}{l}\text { Effective } \\
\text { mechanisms are } \\
\text { in place to } \\
\text { evaluate EHRS } \\
\text { implementation }\end{array}$} & $\mathrm{N}$ & 1 & 1 & 2 & 4 & 2 & 16 & 5 & & 23 & \\
\hline & $\%$ & 3.2 & 3.2 & 6.5 & 12.9 & 6.5 & 51.6 & 16.1 & 6 & 74.2 & 4 \\
\hline \multirow{2}{*}{$\begin{array}{l}\text { Process } \\
\text { improvement } \\
\text { mechanisms are } \\
\text { used effectively to } \\
\text { identify work } \\
\text { process redesign } \\
\text { needs }\end{array}$} & $\mathrm{N}$ & 1 & 5 & 2 & 1 & 4 & 3 & 15 & & 22 & \\
\hline & $\%$ & 3.2 & 16.1 & 6.5 & 3.2 & 12.9 & 9.7 & 48.4 & 6 & 71.0 & 5 \\
\hline \multirow{2}{*}{$\begin{array}{l}\text { EHRS } \\
\text { implementation } \\
\text { decision-makers } \\
\text { are adequately } \\
\text { represented on key } \\
\text { organisational } \\
\text { committees }\end{array}$} & $\mathrm{N}$ & 2 & 2 & & 6 & 1 & 4 & 16 & & 21 & \\
\hline & $\%$ & 6.5 & 6.5 & & 19.4 & 3.2 & 12.9 & 51.6 & 7 & 67.7 & 6 \\
\hline
\end{tabular}




\section{PHCs readiness at the values and goals level}

When examining the values and goals of EHRS implementation, it was found that 1) "Individuals have a positive attitude toward EHRS implementation" had the highest agreement (80.6\%) and a median of six. Similarly, 2) "There is a willingness to engage in the EHRS implementation process" generated the same results of previous item. The least agreement was generated for Item 6) "There is an openness to different perspectives about EHRS implementation", which showed the lowest agreement (54.9\%) (see Table 4.3.10). Corresponding to the objective of this study, Table 4.3.10 shows a good level of PHC readiness for EHRS implementation at values and goals level. 
Table 4.3.10: Participant responses to items representing PHC readiness for EHRS implementation at the values and goals level

\begin{tabular}{|c|c|c|c|c|c|c|c|c|c|c|c|}
\hline $\begin{array}{l}\overrightarrow{\vec{\rho}} \\
\vec{\Xi}\end{array}$ & & 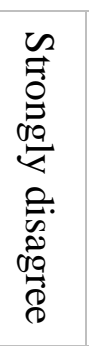 & 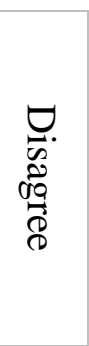 & 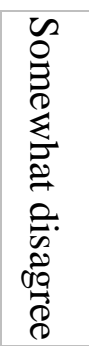 & $\begin{array}{l}z \\
\text { z } \\
o \\
o \\
\text { E. } \\
0 .\end{array}$ & 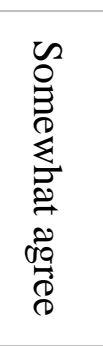 & $\underset{D}{\stackrel{D}{0}}$ & 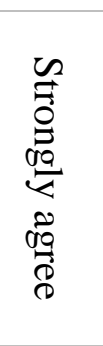 & $\begin{array}{l}\frac{3}{8} \\
\stackrel{0}{0} \\
\text { : }\end{array}$ & 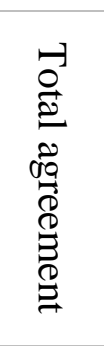 & $\begin{array}{l}\bar{\nabla} \\
\text { 兑 }\end{array}$ \\
\hline \multirow{2}{*}{$\begin{array}{l}\text { Individuals have a } \\
\text { positive attitude } \\
\text { toward EHRS } \\
\text { implementation }\end{array}$} & $\mathrm{N}$ & & 1 & 2 & 3 & 5 & 11 & 9 & \multirow[b]{2}{*}{6} & 25 & \multirow[b]{2}{*}{1} \\
\hline & $\%$ & & 3.2 & 6.5 & 9.7 & 16.1 & 35.5 & 29.0 & & 80.6 & \\
\hline \multirow{2}{*}{$\begin{array}{l}\text { There is a } \\
\text { willingness to } \\
\text { engage in the } \\
\text { EHRS } \\
\text { implementation } \\
\text { process }\end{array}$} & $\mathrm{N}$ & & 2 & 2 & 2 & 1 & 20 & 4 & \multirow[b]{2}{*}{6} & 25 & \multirow[b]{2}{*}{2} \\
\hline & $\%$ & & 6.5 & 6.5 & 6.5 & 3.2 & 64.5 & 12.9 & & 80.6 & \\
\hline \multirow[b]{2}{*}{$\begin{array}{l}\text { There is an } \\
\text { emphasis on the } \\
\text { importance of } \\
\text { collaborative } \\
\text { interdisciplinary } \\
\text { teams to support } \\
\text { EHR } \\
\text { implementation }\end{array}$} & $\mathrm{N}$ & & 2 & 1 & 6 & 3 & 17 & 2 & \multirow[b]{2}{*}{6} & 22 & \multirow[b]{2}{*}{3} \\
\hline & $\%$ & & 6.5 & 3.2 & 19.4 & 9.7 & 54.8 & 6.5 & & 71.0 & \\
\hline \multirow{2}{*}{$\begin{array}{l}\text { There is } \\
\text { satisfaction with } \\
\text { the contribution } \\
\text { that EHRS has } \\
\text { made to the } \\
\text { organisation }\end{array}$} & $\mathrm{N}$ & & 1 & 1 & 9 & 1 & 7 & 12 & \multirow[b]{2}{*}{6} & 20 & \multirow[b]{2}{*}{4} \\
\hline & $\%$ & & 3.2 & 3.2 & 29.0 & 3.2 & 22.6 & 38.7 & & 64.5 & \\
\hline \multirow{2}{*}{$\begin{array}{l}\text { There is a } \\
\text { willingness to act } \\
\text { on work process } \\
\text { improvement } \\
\text { recommendations }\end{array}$} & $\mathrm{N}$ & 1 & 2 & 1 & 9 & 4 & 7 & 7 & \multirow[b]{2}{*}{5} & 18 & \multirow[b]{2}{*}{5} \\
\hline & $\%$ & 3.2 & 6.5 & 3.2 & 29.0 & 12.9 & 22.6 & 22.6 & & 58.1 & \\
\hline \multirow{2}{*}{$\begin{array}{l}\text { There is an } \\
\text { openness to } \\
\text { different } \\
\text { perspectives on } \\
\text { EHRS } \\
\text { implementation }\end{array}$} & $\mathrm{N}$ & & 1 & 2 & 11 & 2 & 13 & 2 & \multirow[b]{2}{*}{5} & 17 & \multirow[b]{2}{*}{6} \\
\hline & $\%$ & & 3.2 & 6.5 & 35.5 & 6.5 & 41.9 & 6.5 & & 54.9 & \\
\hline
\end{tabular}




\section{PHCs readiness at the management structure level}

Table 4.3.11 shows the six items of the scale of PHC readiness at the management structure level. It can be observed that Item 1) "The IT department effectively manages the organisation's shared databases", ranked first with an agreement of $83.9 \%$ and a median of seven, followed by 2) "The business structure supports involvement of IT in strategic planning”, which generated a similar median and an agreement of $77.4 \%$. The least ranked item was 6) "The IT department effectively manages the organisation's shared databases", which had an agreement of $70.9 \%$ and a median of six. 
Table 4.3.11: Participant responses to items representing PHC readiness for EHRS implementation at the management structure level

\begin{tabular}{|c|c|c|c|c|c|c|c|c|c|c|c|}
\hline $\begin{array}{l}\overrightarrow{\overrightarrow{\vec{\theta}}} \\
\overrightarrow{\mathrm{w}_{n}}\end{array}$ & & 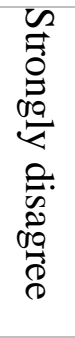 & 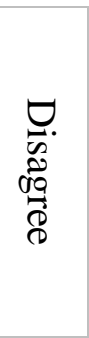 & 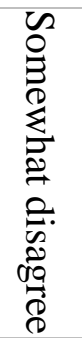 & 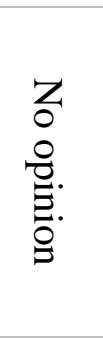 & 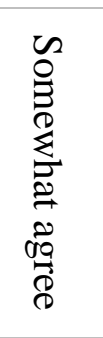 & 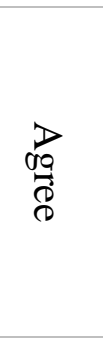 & 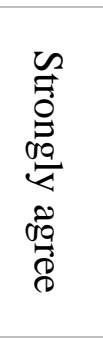 & $\begin{array}{l}3 \\
\frac{1}{2} \\
\stackrel{0}{0}\end{array}$ & 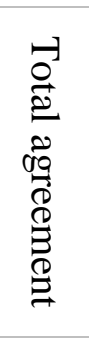 & $\begin{array}{l}\text { त्र } \\
\text { 䒕 }\end{array}$ \\
\hline \multirow{2}{*}{$\begin{array}{l}\text { The IT department } \\
\text { effectively } \\
\text { manages the } \\
\text { organisation's } \\
\text { shared databases }\end{array}$} & $\mathrm{N}$ & & 2 & & 3 & 2 & 4 & 20 & \multirow[b]{2}{*}{7} & 26 & \multirow[b]{2}{*}{1} \\
\hline & $\%$ & & 6.5 & & 9.7 & 6.5 & 12.9 & 64.5 & & 83.9 & \\
\hline \multirow{2}{*}{$\begin{array}{l}\text { The business } \\
\text { structure supports } \\
\text { involvement of IS } \\
\text { in strategic } \\
\text { planning }\end{array}$} & $\mathrm{N}$ & & 2 & 3 & 2 & 4 & 4 & 16 & \multirow[b]{2}{*}{7} & 24 & \multirow[b]{2}{*}{2} \\
\hline & $\%$ & & 6.5 & 9.7 & 6.5 & 12.9 & 12.9 & 51.6 & & 77.4 & \\
\hline \multirow{2}{*}{$\begin{array}{l}\text { Formal policies } \\
\text { and procedures are } \\
\text { available to guide } \\
\text { EHRS } \\
\text { implementation } \\
\text { processes }\end{array}$} & $\mathrm{N}$ & 3 & 1 & 2 & 1 & 2 & 2 & 20 & \multirow[b]{2}{*}{7} & 24 & \multirow[b]{2}{*}{3} \\
\hline & $\%$ & 9.7 & 3.2 & 6.5 & 3.2 & 6.5 & 6.5 & 64.5 & & 77.5 & \\
\hline \multirow[b]{2}{*}{$\begin{array}{l}\text { The IT strategic } \\
\text { plan is an effective } \\
\text { guide for the } \\
\text { organisation's } \\
\text { EHRS } \\
\text { implementation } \\
\text { processes }\end{array}$} & $\mathrm{N}$ & 1 & 3 & 2 & 2 & 2 & 6 & 15 & \multirow[b]{2}{*}{6} & 23 & \multirow[b]{2}{*}{4} \\
\hline & $\%$ & 3.2 & 9.7 & 6.5 & 6.5 & 6.5 & 19.4 & 48.4 & & 74.2 & \\
\hline \multirow[b]{2}{*}{$\begin{array}{l}\text { Formal } \\
\text { communication } \\
\text { mechanisms exist } \\
\text { to support user } \\
\text { and IT support } \\
\text { staff } \\
\text { communication }\end{array}$} & $\mathrm{N}$ & & 3 & 2 & 4 & 2 & 4 & 16 & \multirow[b]{2}{*}{7} & 22 & \multirow[b]{2}{*}{5} \\
\hline & $\%$ & & 9.7 & 6.5 & 12.9 & 6.5 & 12.9 & 51.5 & & 70.9 & \\
\hline \multirow{2}{*}{$\begin{array}{l}\text { The IT department } \\
\text { reporting structure } \\
\text { adequately } \\
\text { supports IT staff }\end{array}$} & N. & & 2 & 3 & 4 & 1 & 9 & 12 & \multirow[b]{2}{*}{6} & 22 & \multirow[b]{2}{*}{6} \\
\hline & $\%$ & & 6.5 & 9.7 & 12.9 & 3.2 & 29.0 & 38.7 & & 70.9 & \\
\hline
\end{tabular}




\section{PHCs readiness at the administrative support level}

All items in the administrative scale generated high agreement (see Table 4.3.12). It should be noted here that some of the items had higher median scores but less agreement than others, hence, the ranking was based on total agreement. The highest ranked item was 1) "Sufficient funds are available to support EHRS implementation planning activities", with a total agreement of $93.6 \%$. The second ranked item is 2) "Executives engage in mutual decision-making with IT leaders regarding proposals and ideas", which had an agreement of 83.9\%. The lowest ranked item was 6) "Sufficient funds are available to support EHRS implementation planning activities", although it also showed high agreement of $70.9 \%$. Corresponding to the objective of this study, Table 4.3.12 shows a high level of PHC readiness for EHRS implementation at the resources level. 
Table 4.3.12: Participant responses to items representing $P H C$ readiness for EHRS implementation at the administration support level

\begin{tabular}{|c|c|c|c|c|c|c|c|c|c|c|c|}
\hline$\underset{\vec{\Phi}}{\vec{\Xi}}$ & & 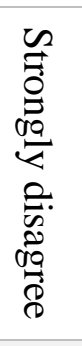 & 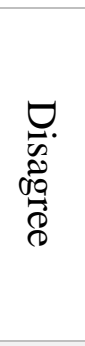 & 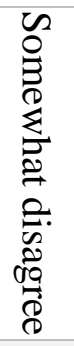 & $\begin{array}{l}Z \\
\text { Z } \\
o \\
o \\
\\
\end{array}$ & 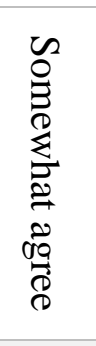 & 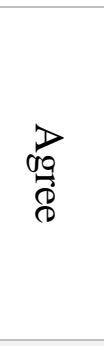 & 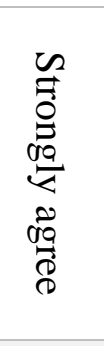 & \begin{tabular}{l}
3 \\
\multirow{2}{0}{} \\
$\stackrel{0}{0}$
\end{tabular} & 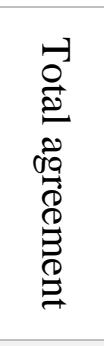 & $\begin{array}{l}\text { त्र } \\
\stackrel{0}{0}\end{array}$ \\
\hline \multirow{2}{*}{$\begin{array}{l}\text { Sufficient funds are } \\
\text { available to support } \\
\text { EHRS } \\
\text { implementation } \\
\text { planning activities }\end{array}$} & $\mathrm{N}$ & & & 1 & 1 & 3 & 8 & 18 & \multirow[b]{2}{*}{7} & 29 & \multirow[b]{2}{*}{1} \\
\hline & $\%$ & & & 3.2 & 3.2 & 9.7 & 25.8 & 58.1 & & 93.6 & \\
\hline \multirow{2}{*}{$\begin{array}{l}\text { Executives engage } \\
\text { in mutual decision- } \\
\text { making with IT } \\
\text { leaders regarding } \\
\text { proposals and ideas }\end{array}$} & $\mathrm{N}$ & & 1 & 1 & 3 & 4 & 7 & 15 & \multirow[b]{2}{*}{6} & 26 & \multirow[b]{2}{*}{2} \\
\hline & $\%$ & & 3.2 & 3.2 & 9.7 & 12.9 & 22.6 & 48.4 & & 83.9 & \\
\hline \multirow{2}{*}{$\begin{array}{l}\text { The top-ranking IT } \\
\text { executive is } \\
\text { regularly included } \\
\text { in senior executive } \\
\text { meetings }\end{array}$} & $\mathrm{N}$ & & 1 & 1 & 4 & & 18 & 7 & \multirow[b]{2}{*}{6} & 25 & \multirow[b]{2}{*}{3} \\
\hline & $\%$ & & 3.2 & 3.2 & 12.9 & & 58.1 & 22.6 & & 80.7 & \\
\hline \multirow[b]{2}{*}{$\begin{array}{l}\text { EHRS } \\
\text { implementation } \\
\text { initiatives are } \\
\text { usually addressed } \\
\text { as part of the } \\
\text { organisation's } \\
\text { overall strategic } \\
\text { planning }\end{array}$} & $\mathrm{N}$ & 2 & 1 & 1 & 3 & 2 & 7 & 15 & \multirow[b]{2}{*}{6} & 24 & \multirow[b]{2}{*}{4} \\
\hline & $\%$ & 6.5 & 3.2 & 3.2 & 9.7 & 6.5 & 22.6 & 48.4 & & 77.5 & \\
\hline \multirow{2}{*}{$\begin{array}{l}\text { Non-IT executives } \\
\text { are routinely named } \\
\text { as co-sponsors for } \\
\text { EHRS } \\
\text { implementation } \\
\text { projects }\end{array}$} & $\mathrm{N}$ & & 1 & 3 & 3 & 4 & 15 & 5 & \multirow[b]{2}{*}{6} & 24 & \multirow[b]{2}{*}{5} \\
\hline & $\%$ & & 3.2 & 9.7 & 9.7 & 12.9 & 48.4 & 16.1 & & 77.4 & \\
\hline \multirow{2}{*}{$\begin{array}{l}\text { Board members are } \\
\text { actively engaged in } \\
\text { key EHRS } \\
\text { implementation } \\
\text { strategic plan } \\
\text { committees }\end{array}$} & $\mathrm{N}$ & & 3 & 1 & 5 & 1 & 4 & 17 & \multirow[b]{2}{*}{7} & 22 & \multirow[b]{2}{*}{6} \\
\hline & $\%$ & & 9.7 & 3.2 & 16.1 & 3.2 & 12.9 & 54.8 & & 70.9 & \\
\hline
\end{tabular}

In response to the first objective of this study, the above eight tables show a high level of PHC readiness in all examined elements. Although the above tables reveal a high level of 
PHC readiness in all factors, only process, management structure and administration support achieved the minimum median score of six (agree). All items in the three scales are categorised as organisational factors. These aspects will be further discussed later in this thesis.

\subsubsection{The impact of CM on EHRS implementation PHCs in SA:}

Similar to the previous section, items in this scale are ranked based on total agreement. This scale looked at whether the Saudi MoH CM had any impact on EHRS implementation in PHCs. This scale was designed to correspond to the second objective of this study, which is to evaluate the impact of CM and financial resources on EHRS implementation in PHCs in SA. The impact was explored with nine items (see Table 4.3.13). Overall, it was shown that there is an extremely high agreement on all items (above 90\%), with median scores being six or seven. Item 1) "Overall impact is positive", showed an agreement of $96.8 \%$ and a median score of seven, where participants strongly agree that CM positively influences EHRS implementation in PHCs in SA. Whereas the lowest rank was given to item 9) "Improve project team communication" (90.4\%), which is also considered very high with median score of six. 
Table 4.3.13: Participant responses to items representing the impact of CM on EHRS implementation in PHCs

\begin{tabular}{|c|c|c|c|c|c|c|c|c|c|c|c|}
\hline$\underset{\vec{\Phi}}{\vec{\Xi}}$ & & 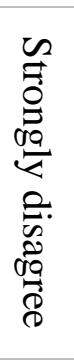 & 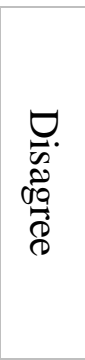 & 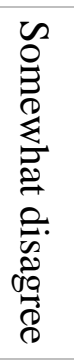 & $\begin{array}{l}z \\
0 \\
0 \\
o \\
0 . \\
0 . \\
0\end{array}$ & 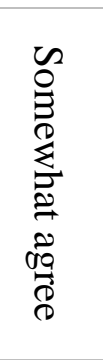 & 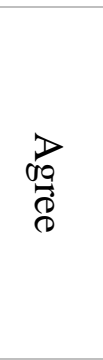 & 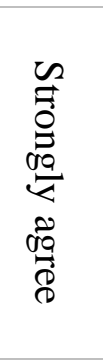 & 胥. & 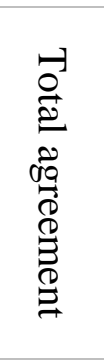 & 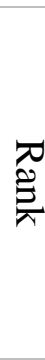 \\
\hline \multirow{2}{*}{$\begin{array}{l}\text { Overall impact is } \\
\text { positive }\end{array}$} & $\mathrm{N}$ & & & & 1 & & 5 & 25 & \multirow{2}{*}{7} & 30 & \multirow{2}{*}{1} \\
\hline & $\%$ & & & & 3.2 & & 16.1 & 80.6 & & 96.8 & \\
\hline \multirow{2}{*}{$\begin{array}{l}\text { Improve systems } \\
\text { integration and } \\
\text { interoperability }\end{array}$} & $\mathrm{N}$ & & & & 1 & & 12 & 18 & \multirow[b]{2}{*}{7} & 30 & \multirow[b]{2}{*}{2} \\
\hline & $\%$ & & & & 3.2 & & 38.7 & 58.1 & & 96.8 & \\
\hline \multirow{2}{*}{$\begin{array}{l}\text { Better software } \\
\text { selection }\end{array}$} & $\mathrm{N}$ & & 1 & & 1 & 1 & 7 & 21 & \multirow{2}{*}{7} & 29 & \multirow{2}{*}{3} \\
\hline & $\%$ & & 3.2 & & 3.2 & 3.2 & 22.6 & 67.7 & & 93.5 & \\
\hline \multirow{2}{*}{$\begin{array}{l}\text { Easier to manage } \\
\text { EHRS } \\
\text { implementation in } \\
\text { a large number of } \\
\text { PHCs that are } \\
\text { widely dispersed }\end{array}$} & $\mathrm{N}$ & & & 1 & 1 & & 12 & 17 & \multirow[b]{2}{*}{7} & 29 & \\
\hline & $\%$ & & & 3.2 & 3.2 & & 38.7 & 54.8 & & 93.5 & 4 \\
\hline \multirow{2}{*}{$\begin{array}{l}\text { Better project } \\
\text { team selection }\end{array}$} & $\mathrm{N}$ & & & & 2 & 4 & 8 & 17 & \multirow{2}{*}{7} & 29 & \multirow{2}{*}{5} \\
\hline & $\%$ & & & & 6.5 & 12.9 & 25.8 & 54.8 & & 93.5 & \\
\hline \multirow{2}{*}{$\begin{array}{l}\text { Better decision- } \\
\text { making }\end{array}$} & $\mathrm{N}$ & & 1 & & 2 & & 6 & 22 & \multirow{2}{*}{7} & 28 & \multirow{2}{*}{6} \\
\hline & $\%$ & & 3.2 & & 6.5 & & 19.4 & 71.0 & & 90.4 & \\
\hline \multirow{2}{*}{$\begin{array}{l}\text { Improve } \\
\text { implementation of } \\
\text { the strategic plan }\end{array}$} & $\mathrm{N}$ & & 1 & & 2 & 1 & 9 & 18 & \multirow[b]{2}{*}{7} & 28 & \multirow[b]{2}{*}{7} \\
\hline & $\%$ & & 3.2 & & 6.5 & 3.2 & 29.0 & 58.1 & & 90.4 & \\
\hline \multirow{2}{*}{$\begin{array}{l}\text { Help leading and } \\
\text { managing the } \\
\text { project }\end{array}$} & $\mathrm{N}$ & & & 1 & 1 & 3 & 10 & 15 & \multirow[b]{2}{*}{6.50} & 28 & \multirow[b]{2}{*}{8} \\
\hline & $\%$ & & & 3.2 & 3.2 & 9.7 & 32.3 & 48.4 & & 90.4 & \\
\hline \multirow{2}{*}{$\begin{array}{l}\text { Improve project } \\
\text { team } \\
\text { communication }\end{array}$} & $\mathrm{N}$ & & & & 3 & 1 & 18 & 9 & \multirow[b]{2}{*}{6} & 28 & \multirow[b]{2}{*}{9} \\
\hline & $\%$ & & & & 9.7 & 3.2 & 58.1 & 29.0 & & 90.4 & \\
\hline
\end{tabular}




\subsubsection{The impact of financial resources (FR) on EHRS implementation in PHCs in SA}

Similar to the above, the items in this scale were ranked based on total agreement. This scale was designed to achieve the second objective of this study and evaluate the impact of FR on EHRS implementation in PHCs in SA. The impact was explored with eleven items (see Table 4.3.14). Overall, it was shown that there is a high agreement on all items (above $80 \%$ ), some of which had slightly more agreement than others, with median scores being six or seven. It was shown that the influence of an abundance of financial resources is 1) "overall positive”, showing an agreement of $96.8 \%$ and a median score of seven, where participants strongly agree that an abundance of financial resources positively influences EHRS implementation. Whereas the least agreement was given for Item 11) "Improve systems integration and interoperability" (83.9\%). Participants were asked whether the FR has a positive impact on the availability of sufficient project team members and other individuals or not "Abundance of staff and professionals", responses were mostly agree $(87,1 \%)$ 
Table 4.3.14: Participant responses to items representing the impact of financial resources on EHRS implementation in PHCs

\begin{tabular}{|c|c|c|c|c|c|c|c|c|c|c|c|}
\hline \multicolumn{2}{|l|}{$\overrightarrow{\vec{\rho}}$} & 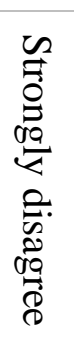 & 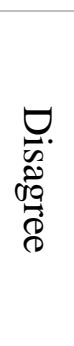 & 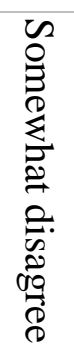 & 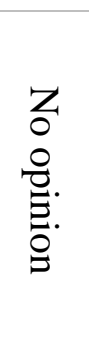 & 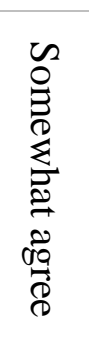 & $\underset{\substack{\infty \\
0}}{\stackrel{\infty}{8}}$ & 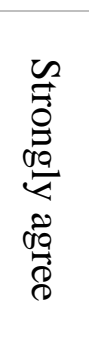 & : & 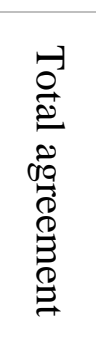 & 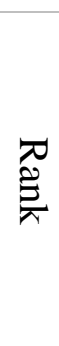 \\
\hline \multirow{2}{*}{$\begin{array}{l}\text { Overall impact is } \\
\text { positive }\end{array}$} & $\mathrm{N}$ & & & & 1 & 2 & 3 & 25 & \multirow{2}{*}{7} & 30 & \multirow{2}{*}{1} \\
\hline & $\%$ & & & & 3.2 & 6.5 & 9.7 & 80.6 & & 96.8 & \\
\hline \multirow{2}{*}{$\begin{array}{l}\text { Better software } \\
\text { selection }\end{array}$} & $\mathrm{N}$ & & & & 2 & 2 & 8 & 19 & \multirow{2}{*}{7} & 29 & \multirow{2}{*}{2} \\
\hline & $\%$ & & & & 6.5 & 6.5 & 25.8 & 61.3 & & 93.5 & \\
\hline \multirow{2}{*}{$\begin{array}{l}\text { Better team } \\
\text { selection }\end{array}$} & $\mathrm{N}$ & & & & 2 & 4 & 14 & 11 & \multirow{2}{*}{6} & 29 & \multirow{2}{*}{3} \\
\hline & $\%$ & & & & 6.5 & 12.9 & 45.2 & 35.5 & & 93.5 & \\
\hline \multirow{2}{*}{$\begin{array}{l}\text { Improve user } \\
\text { training and } \\
\text { motivation }\end{array}$} & $\mathrm{N}$ & & 1 & 1 & 1 & 1 & 5 & 22 & \multirow[b]{2}{*}{7} & 28 & \multirow[b]{2}{*}{4} \\
\hline & $\%$ & & 3.2 & 3.2 & 3.2 & 3.2 & 16.1 & 71.0 & & 90.3 & \\
\hline Improve the & $\mathrm{N}$ & & & & 3 & 1 & 8 & 19 & \multirow[b]{2}{*}{7} & 28 & \multirow[b]{2}{*}{5} \\
\hline $\begin{array}{l}\text { provision of } \\
\text { appropriate } \\
\text { hardware }\end{array}$ & $\%$ & & & & 9.7 & 3.2 & 25.8 & 61.3 & & 90.3 & \\
\hline \multirow{2}{*}{$\begin{array}{l}\text { Improve on-going } \\
\text { support and } \\
\text { maintenance }\end{array}$} & $\mathrm{N}$ & & & & 4 & 2 & 6 & 19 & \multirow[b]{2}{*}{7} & 27 & \multirow[b]{2}{*}{6} \\
\hline & $\%$ & & & & 12.9 & 6.5 & 19.4 & 61.3 & & 87.1 & \\
\hline \multirow{2}{*}{$\begin{array}{l}\text { Abundance of } \\
\text { staff and } \\
\text { professionals }\end{array}$} & $\mathrm{N}$ & & 1 & 2 & 1 & 4 & 4 & 19 & \multirow[b]{2}{*}{7} & 27 & \multirow[b]{2}{*}{7} \\
\hline & $\%$ & & 3.2 & 6.5 & 3.2 & 12.9 & 12.9 & 61.3 & & 87.1 & \\
\hline \multirow{2}{*}{$\begin{array}{l}\text { Appropriate } \\
\text { infrastructure }\end{array}$} & $\mathrm{N}$ & & & 2 & 2 & 1 & 8 & 18 & \multirow{2}{*}{7} & 27 & \multirow{2}{*}{8} \\
\hline & $\%$ & & & 6.5 & 6.5 & 3.2 & 25.8 & 58.1 & & 87.1 & \\
\hline \multirow{2}{*}{$\begin{array}{l}\text { Improve team } \\
\text { communication }\end{array}$} & $\mathrm{N}$ & 1 & 1 & & 2 & 3 & 14 & 10 & \multirow{2}{*}{6.} & 27 & \multirow{2}{*}{9} \\
\hline & $\%$ & 3.2 & 3.2 & & 6.5 & 9.7 & 45.2 & 32.3 & & 87.1 & \\
\hline \multirow{2}{*}{$\begin{array}{l}\text { Improve systems } \\
\text { integration and } \\
\text { interoperability }\end{array}$} & $\mathrm{N}$ & 1 & & 2 & 2 & 1 & 9 & 16 & \multirow[b]{2}{*}{7} & 26 & \multirow[b]{2}{*}{10} \\
\hline & $\%$ & 3.2 & & 6.5 & 6.5 & 3.2 & 29.0 & 51.6 & & 83.9 & \\
\hline \multirow{2}{*}{$\begin{array}{l}\text { Improve } \\
\text { organisation } \\
\text { workflow and } \\
\text { structure redesign }\end{array}$} & $\mathrm{N}$ & 1 & 2 & 1 & 1 & 2 & 15 & 9 & \multirow[b]{2}{*}{6} & 26 & \\
\hline & $\%$ & 3.2 & 6.5 & 3.2 & 3.2 & 6.5 & 48.4 & 29.0 & & 83.9 & 11 \\
\hline
\end{tabular}




\subsection{Summary}

Only thirty-one questionnaires of the fifty-three distributed to the EHRS implementation project team at the Saudi $\mathrm{MoH}$ were returned. Over $80 \%$ of the participants were male and the majority of the participants occupied assistant positions (67.7\%). About half of the participants had previous involvement in similar EHRS implementation projects, and $64.5 \%$ of the participants had been directly involved in the EHRS implementation in PHCs in SA. Overall, the participant responses show that the Saudi PHCs are high level of readiness for EHRS implementation. PHCs readiness was very high at the process, management structure and administration support levels, while readiness was good at end-user, technology and values and goals levels.

As this study was aiming to evaluate the impact of CM and FR on EHRS implementation in the PHCs in SA, the most obvious finding to emerge from this study is that FR has a very positive impact on implementation. According to the total agreement and ranking tests, the most influenced factor by the FR was the software selection (will be explored in Chapter Eight). Likewise, CM was recorded to have a positive impact on EHRS implementation projects in Saudi PHCs. According to the total agreement and ranking tests, the most influenced factor by the CM was the EHRS interoperability (will be explored in Chapter Eight).

The following chapter outlines findings corresponding to objectives of the current study related to the readiness of Saudi PHCs to implement a new EHRS. However, the findings in the following study were obtained from PHC staff via electronic questionnaire-based research. 
5 Chapter Five: Study Two: Assessing PHCs Readiness for EHRS Implementation in SA from a PHC Staff Perspective 


\subsection{Introduction}

This chapter presents the results and statistics of the study in assessing the readiness of Saudi PHCs for EHRS implementation from a PHC staff perspective. To achieve the study aim, two different statistical tests were performed. Firstly, a descriptive statistics test was carried out to determine the overall level of PHCs readiness for the implementation of an EHRS. Items in the questionnaire have been described and then classified into two main scales. Once all items were allocated to their appropriate scales, a Cronbach's Alpha test was performed to ensure all main scales are reliable enough to conduct inferential statistics. Thereafter, inferential statistics were performed to determine the impact of demographic differences of the participants on individual readiness for EHRS implementation. As previously revealed, individual demographics, such as experience with using a PC, may make a difference regarding readiness for EHRS implementation (see Chapter Two).

\subsection{Scale reliability}

The reliability of the benefits and readiness scale was measured using a Cronbach's Alpha test. The results of the test are reflected in Table 5.2.1 below, which shows good internal consistency between the items in each of the scales (themes). The readiness scale reflected a score of $89 \%$ consistency (0.89) and the perceived usefulness of an EHRS scale a score of $94 \%(0.94)$. As a result, it can be concluded that both scales are reliable.

Table 5.2.1: Reliability of benefits and readiness scales

\begin{tabular}{|l|c|c|}
\multicolumn{1}{|c|}{ Scale } & $\begin{array}{c}\text { Number of } \\
\text { Items }\end{array}$ & $\begin{array}{c}\text { Cronbach's } \\
\text { Alpha }\end{array}$ \\
\hline $\begin{array}{l}\text { PHCs staff awareness about perceived } \\
\text { usefulness of EHRS }\end{array}$ & 13 & 0.94 \\
PHCs readiness & & 0.89 \\
\hline Entire questionnaire & 13 & $\mathbf{0 . 9 3}$ \\
\hline
\end{tabular}




\subsection{Descriptive statistics of participant responses}

Out of the 491 healthcare practitioners working in the selected PHCs, 351 completed in the questionnaire. This equated to a response rate of $71.5 \%$. Therefore, the questionnaire data were collected from 351 participants across five different regions of the Kingdom of Saudi Arabia. The largest number of the respondents, 103 (29.3\%), were residents of the capital city, Riyadh (see Table 5.3.1). As seen the below table, the majority of participants were from Riyadh and Makkah regions which reflect the number of populations in these regions as the biggest regions in SA.

Table 5.3.1: Participant distribution based on geographical location

\begin{tabular}{|l|c|c|}
\hline Region & Frequency & Percent \\
\hline Riyadh & 103 & 29.3 \\
\hline Gassim & 61 & 17.4 \\
\hline Aljouf & 69 & 19.7 \\
\hline Albaha & 30 & 8.5 \\
\hline Makkah & 88 & 25.1 \\
\hline Total & 351 & 100.0 \\
\hline
\end{tabular}

All participants worked in healthcare and administrative roles. As can be seen in Table 5.3.2, $149(42.4 \%)$ were in a administrators role such as managers, secretaries and receptionist, $104(29.6 \%)$ worked in a nursing role, thirty-two (9.1\%) were physicians and thirty $(8.5 \%)$ were pharmacists. Four $(1.1 \%)$ participants did not declare their occupation. The below table show that the study sample is representative and reflect the actual portion of the study population $(\mathrm{MoH}, 2012)$.

Table 5.3.2: Participant distribution based on occupation

\begin{tabular}{|l|c|c|}
\hline Occupation & Frequency & Percent \\
\hline Administrator & 149 & 42.4 \\
\hline Physician & 32 & 9.1 \\
\hline Nurse & 104 & 29.6 \\
\hline Lap technician & 11 & 3.1 \\
\hline Pharmacist & 30 & 8.5 \\
\hline Radiologist & 9 & 2.6 \\
\hline Dentist & 12 & 3.4 \\
\hline Total & 347 & 98.9 \\
\hline
\end{tabular}


Age was measured via six categories, as illustrated in Table 5.3.3 below. As revealed in Chapter Two, age differences have an influence on the level of individual readiness. The majority of participants, 192 (54.7\%), were between twenty-five and thirty-four years of age. A detailed breakdown of the age categories is provided in Table 5.3.3. Four (1.1\%) participants did not declare their age. The below table show that only $29(8.3 \%)$ of the participants are over 45 years old.

Table 5.3.3: Participant distribution based on age

\begin{tabular}{|l|c|c|}
\hline Age Group & Frequency & Percent \\
\hline 18 to 24 & 3 & .9 \\
\hline 25 to 34 & 192 & 54.7 \\
\hline 35 to 44 & 123 & 35.0 \\
\hline 45 to 54 & 23 & 6.6 \\
\hline 55 to 64 & 4 & 1.1 \\
\hline 65 to 74 & 2 & .6 \\
\hline Total & 347 & 98.9 \\
\hline
\end{tabular}

Participants were asked to specify their gender, as shown in Table 5.3.4. Participants were mostly male ( $\mathrm{n}=261 ; 74.4 \%)$. Out of 351 participants, only eighty-one $(23.1 \%)$ were female. This number reflect the actual ratio of males against females in the PHCs in SA $(\mathrm{MoH}, 2012)$. Nine (2.6\%) participants did not declare their gender.

Table 5.3.4: Participant distribution based on gender

\begin{tabular}{|c|c|c|}
\hline Gender & Frequency & Percent \\
\hline Female & 81 & 23.1 \\
\hline Male & 261 & 74.4 \\
\hline Total & 342 & 97.4 \\
\hline
\end{tabular}

The participants' experience of using a Personal Computer (PC) at home varied, with most participants, 129 (36.8\%), stating that they had experience ranging between ten to fifteen years. Only eighteen participants $(5.1 \%)$ had less than one year's experience with using a PC (see Table 5.3.5). As revealed in Chapter Two, experience with computer consider as a core predictor to the level of individual readiness. Therefore, the below table indicates that the PHCs staff at high level of readiness, whereas the majority of PHCs had 
more than five years' experience in using PC (will be discussed in Chapter Eight). Four (1.1\%) participants did not declare their experience with using a PC at home.

Table 5.3.5: Participant distribution based on their experience with using a personal computer

\begin{tabular}{|l|c|c|}
\hline Length of experience & Frequency & Percent \\
\hline Less than 1 year & 18 & 5.1 \\
\hline 1 to 5 years & 29 & 8.3 \\
\hline 5 to 10 years & 109 & 31.1 \\
\hline 10 - 15 years & 129 & 36.8 \\
\hline More than 20 years & 62 & 17.7 \\
\hline Total & 347 & 98.9 \\
\hline
\end{tabular}

The participants' time spent working in their current work role was measured via five periodic categories. The majority of participants, 105 (29.9\%), had one to five years' experience. A detailed breakdown of participant's time in their current position is provided in Table 5.3.6. As seen in the below table, most of the participants had one-year experience and more, only $19(5.4 \%)$ had less than one year in their current position. Five (1.4\%) participants did not declare their experience in their current position.

Table 5.3.6: Participant distribution based on experience in their current position

\begin{tabular}{|l|c|c|}
\hline Length of experience & Frequency & Percent \\
\hline Less than 1 year & 19 & 5.4 \\
\hline 1 to 5 years & 105 & 29.9 \\
\hline 5 to 10 years & 100 & 28.5 \\
\hline 10 to 15 years & 82 & 23.4 \\
\hline More than 20 years & 40 & 11.4 \\
\hline Total & 346 & 98.6 \\
\hline
\end{tabular}

\subsubsection{Participant perceptions of the perceived usefulness of an EHRS}

In order to determine the participant awareness and perceptions of the usefulness of an EHRS, they were asked to answer thirteen items reflecting the possible benefits of an EHRS. All the items generated high agreement scores, ranging from $90.5 \%$ to $84.2 \%$ (see Table 5.3.7). This clearly indicates that most of the participants agree with all the 
statements on the questionnaire. It was observed that the highest ranked benefit was "Information from the EHRS enables me to make better decisions about patient care" (90.5\%), followed by "EHRS provides accurate, up-to-date and complete information about patients at the point of care" (89.9\%), "EHRS enable quick access to patient records for more coordinated, efficient care" (89.5\%), and "EHRS improve patient and healthcare professionals interaction and communication as well as healthcare convenience" (89.0\%). At the bottom of the scale, though still with high agreement, the least ranked items were "EHRS improve end-users' productivity and efficiency" (87.3\%), "EHRS enable safer, more reliable prescribing" (87.0\%), "EHRS improve the privacy and security of patient data" (85.8\%) and, finally, "Using the EHRS helps to reduce medical errors" (84.2\%). Overall, participants express high level of awareness about the perceived usefulness of the EHRS. 
Table 5.3.7: Participant responses to statements regarding PHC staff awareness of the perceived usefulness of an EHRS $(N=13)$

\begin{tabular}{|c|c|c|c|c|c|c|c|c|c|}
\hline$\underset{\widetilde{\Xi}}{\overparen{s}}$ & & 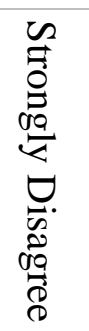 & 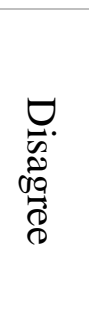 & 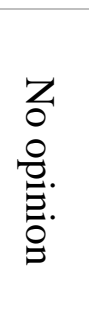 & $\underset{\substack{90 \\
8}}{\frac{D}{8}}$ & 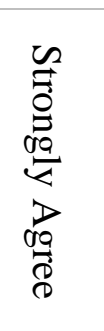 & 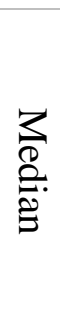 & 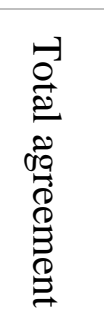 & 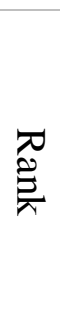 \\
\hline \multirow{2}{*}{$\begin{array}{l}\text { Information from EHRS } \\
\text { enables better decisions } \\
\text { about patient care }\end{array}$} & $\mathrm{N}$ & 22 & 8 & 3 & 154 & 160 & \multirow[b]{2}{*}{4} & 314 & \multirow[b]{2}{*}{1} \\
\hline & $\%$ & 6.3 & 2.3 & .9 & 44.4 & 46.1 & & 90.5 & \\
\hline \multirow{2}{*}{$\begin{array}{l}\text { EHRS provide accurate, } \\
\text { up-to-date and complete } \\
\text { information about patients } \\
\text { at the point of care }\end{array}$} & $\mathrm{N}$ & 19 & 13 & 3 & 160 & 150 & \multirow[b]{2}{*}{4} & 310 & \multirow[b]{2}{*}{2} \\
\hline & $\%$ & 5.5 & 3.8 & .9 & 46.4 & 43.5 & & 89.9 & \\
\hline \multirow{2}{*}{$\begin{array}{l}\text { EHRS enables quick access } \\
\text { to patient records for more } \\
\text { co-ordinated, efficient care }\end{array}$} & $\mathrm{N}$ & 23 & 9 & 4 & 123 & 184 & \multirow[b]{2}{*}{5} & 307 & \multirow[b]{2}{*}{3} \\
\hline & $\%$ & 6.7 & 2.6 & 1.2 & 35.9 & 53.6 & & 89.5 & \\
\hline \multirow{2}{*}{$\begin{array}{l}\text { EHRS improves patient } \\
\text { and healthcare } \\
\text { professionals' interaction } \\
\text { and communication as well } \\
\text { as healthcare convenience }\end{array}$} & $\mathrm{N}$ & 24 & 8 & 6 & 122 & 185 & \multirow[b]{2}{*}{5} & 307 & \multirow[b]{2}{*}{4} \\
\hline & $\%$ & 7.0 & 2.3 & 1.7 & 35.4 & 53.6 & & 89.0 & \\
\hline \multirow{2}{*}{$\begin{array}{l}\text { Using EHRS helps to } \\
\text { effectively diagnose } \\
\text { patients }\end{array}$} & $\mathrm{N}$ & 21 & 15 & 4 & 137 & 169 & \multirow[b]{2}{*}{4} & 306 & \multirow[b]{2}{*}{5} \\
\hline & $\%$ & 6.1 & 4.3 & 1.2 & 39.6 & 48.8 & & 88.4 & \\
\hline \multirow{2}{*}{$\begin{array}{l}\text { EHRS reduces costs } \\
\text { through decreased } \\
\text { paperwork, improved } \\
\text { safety, reduced duplication } \\
\text { of testing and improved } \\
\text { health }\end{array}$} & $\mathrm{N}$ & 24 & 12 & 4 & 108 & 195 & \multirow[b]{2}{*}{5} & 303 & \multirow[b]{2}{*}{6} \\
\hline & $\%$ & 7.0 & 3.5 & 1.2 & 31.5 & 56.9 & & 88.4 & \\
\hline \multirow{2}{*}{$\begin{array}{l}\text { EHRS helps to promote } \\
\text { legible, complete } \\
\text { documentation and } \\
\text { accurate, streamlined } \\
\text { coding and billing. }\end{array}$} & $\mathrm{N}$ & 24 & 12 & 5 & 113 & 191 & \multirow[b]{2}{*}{5} & 304 & \multirow[b]{2}{*}{7} \\
\hline & $\%$ & 7.0 & 3.5 & 1.4 & 32.8 & 55.4 & & 88.2 & \\
\hline \multirow{2}{*}{$\begin{array}{l}\text { Sharing electronic } \\
\text { information with patients } \\
\text { and other clinicians is } \\
\text { more secure when using an } \\
\text { EHRS }\end{array}$} & $\mathrm{N}$ & 24 & 15 & 4 & 134 & 167 & \multirow[b]{2}{*}{4} & 301 & \multirow[b]{2}{*}{8} \\
\hline & $\%$ & 7.0 & 4.4 & 1.2 & 39.0 & 48.5 & & 87.5 & \\
\hline \multirow{2}{*}{$\begin{array}{l}\text { An EHRS helps to provide } \\
\text { safer care }\end{array}$} & $\mathrm{N}$ & 23 & 16 & 5 & 128 & 174 & \multirow{2}{*}{5} & 302 & \multirow{2}{*}{9} \\
\hline & $\%$ & 6.6 & 4.6 & 1.4 & 37.0 & 50.3 & & 87.3 & \\
\hline \multirow{2}{*}{$\begin{array}{l}\text { EHRS improves end-user } \\
\text { productivity and efficiency }\end{array}$} & $\mathrm{N}$ & 24 & 15 & 5 & 121 & 180 & \multirow{2}{*}{5} & 301 & \multirow{2}{*}{10} \\
\hline & $\%$ & 7.0 & 4.3 & 1.4 & 35.1 & 52.2 & & 87.3 & \\
\hline \multirow{2}{*}{$\begin{array}{l}\text { EHRS enables safer, more } \\
\text { reliable prescribing. }\end{array}$} & $\mathrm{N}$ & 22 & 15 & 8 & 109 & 191 & \multirow{2}{*}{5} & 300 & \multirow{2}{*}{11} \\
\hline & $\%$ & 6.4 & 4.3 & 2.3 & 31.6 & 55.4 & & 87.0 & \\
\hline
\end{tabular}




\begin{tabular}{|l|c|c|c|c|c|c|c|c|c|}
\hline $\begin{array}{l}\text { EHRS improves the privacy } \\
\text { and security of patient data }\end{array}$ & $\mathrm{N}$ & 22 & 22 & 5 & 110 & 185 & 5 & 295 & 12 \\
\hline $\begin{array}{l}\text { EHRS helps to reduce } \\
\text { medical errors }\end{array}$ & $\mathrm{N}$ & 25 & 6.4 & 1.5 & 32.0 & 53.8 & & 85.8 & \\
\hline
\end{tabular}

\subsubsection{Participant responses regarding perceived readiness for EHRS implementation}

This section assesses the perceived level of readiness for EHRS implementation. The most important aspect of readiness, according to the questionnaire, was that "Individuals have a positive attitude toward EHR implementation" (84.1\%), which generated the highest total agreement, followed by "Users are typically supportive of EHRS" (82.5\%), "There is a willingness to engage in the EHR implementation process" (82.2\%), and "Most users have an adequate level of computer literacy" (74.1\%). At the other end of the scale, there was more disagreement than agreement on a number of items, mainly those concerning formal communication, specifically "Formal communication mechanisms exist to support user and IT support staff communication" (22.2\%), followed by "Adequate communication mechanisms exist to support shared communication across all organisational levels" (19.8\%), and "Staff have been included in decision-making processes" (18\%). Finally, the least agreed upon item stated that, "Current work practices are adequately supported by existing information systems" (15.4\%) (see Table 5.3.8). 
Table 5.3.8: Participant responses regarding the perceived readiness of PHCs to implement an EHRS $(N=13)$

\begin{tabular}{|c|c|c|c|c|c|c|c|c|c|}
\hline 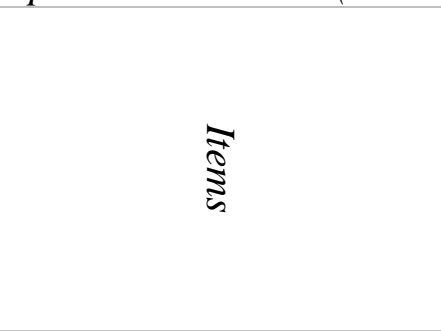 & & 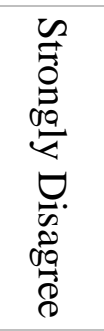 & 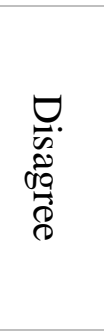 & $\begin{array}{l}z \\
0 \\
0 \\
o \\
0 . \\
0 \\
0\end{array}$ & $\underset{\substack{\infty \\
\mathbb{8}}}{\mathbb{8}}$ & 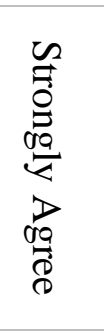 & : & 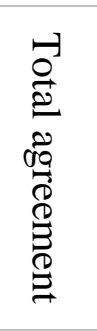 & $\underset{\widetilde{D}}{\bar{\lambda}}$ \\
\hline \multirow{2}{*}{$\begin{array}{l}\text { Individuals have a } \\
\text { positive attitude toward } \\
\text { EHRS implementation }\end{array}$} & $\mathrm{N}$ & 19 & 30 & 5 & 116 & 171 & \multirow{2}{*}{5} & 287 & \multirow[b]{2}{*}{1} \\
\hline & $\%$ & 5.6 & 8.8 & 1.5 & 34.0 & 50.1 & & 84.1 & \\
\hline \multirow{2}{*}{$\begin{array}{l}\text { Users are typically } \\
\text { supportive of an EHRS }\end{array}$} & $\mathrm{N}$ & 18 & 39 & 4 & 164 & 122 & \multirow{2}{*}{4} & 286 & \multirow{2}{*}{2} \\
\hline & $\%$ & 5.2 & 11.2 & 1.2 & 47.3 & 35.2 & & 82.5 & \\
\hline \multirow{2}{*}{$\begin{array}{l}\text { There is a willingness to } \\
\text { engage in the EHRS } \\
\text { implementation process }\end{array}$} & $\mathrm{N}$ & 23 & 32 & 6 & 114 & 168 & \multirow[b]{2}{*}{4} & 282 & \multirow[b]{2}{*}{3} \\
\hline & $\%$ & 6.7 & 9.3 & 1.7 & 33.2 & 49.0 & & 82.2 & \\
\hline \multirow{2}{*}{$\begin{array}{l}\text { Most users have an } \\
\text { adequate level of } \\
\text { computer literacy }\end{array}$} & $\mathrm{N}$ & 27 & 59 & 4 & 160 & 97 & \multirow[b]{2}{*}{4} & 257 & \multirow[b]{2}{*}{4} \\
\hline & $\%$ & 7.8 & 17.0 & 1.2 & 46.1 & 28.0 & & 74.1 & \\
\hline \multirow{2}{*}{$\begin{array}{l}\text { Staff are typically } \\
\text { involved in EHRS } \\
\text { implementation. }\end{array}$} & $\mathrm{N}$ & 72 & 140 & 19 & 77 & 36 & \multirow[b]{2}{*}{2} & 113 & \multirow[b]{2}{*}{5} \\
\hline & $\%$ & 20.9 & 40.7 & 5.5 & 22.4 & 10.5 & & 32.9 & \\
\hline \multirow{2}{*}{$\begin{array}{l}\text { Adequate training is } \\
\text { available to support } \\
\text { users }\end{array}$} & $\mathrm{N}$ & 75 & 145 & 16 & 60 & 49 & \multirow[b]{2}{*}{2} & 109 & \multirow[b]{2}{*}{6} \\
\hline & $\%$ & 21.7 & 42.0 & 4.6 & 17.4 & 14.2 & & 31.6 & \\
\hline \multirow[b]{2}{*}{$\begin{array}{l}\text { There is an emphasis on } \\
\text { the importance of } \\
\text { collaborative } \\
\text { interdisciplinary teams } \\
\text { to support EHRS } \\
\text { implementation }\end{array}$} & $\mathrm{N}$ & 89 & 149 & 21 & 55 & 29 & \multirow[b]{2}{*}{2} & 84 & \multirow[b]{2}{*}{7} \\
\hline & $\%$ & 25.9 & 43.4 & 6.1 & 16.0 & 8.5 & & 24.5 & \\
\hline \multirow{2}{*}{$\begin{array}{l}\text { A core group of users } \\
\text { (champions) is available } \\
\text { to support } \\
\text { implementation }\end{array}$} & $\mathrm{N}$ & 85 & 154 & 26 & 52 & 28 & \multirow[b]{2}{*}{2} & 80 & \multirow[b]{2}{*}{8} \\
\hline & $\%$ & 24.6 & 44.6 & 7.5 & 15.1 & 8.1 & & 23.2 & \\
\hline \multirow{2}{*}{$\begin{array}{l}\text { Knowledge about how } \\
\text { EHRS is being used by } \\
\text { other organisations is } \\
\text { available }\end{array}$} & $\mathrm{N}$ & 92 & 158 & 15 & 55 & 24 & \multirow[b]{2}{*}{2} & 79 & \multirow[b]{2}{*}{9} \\
\hline & $\%$ & 26.7 & 45.9 & 4.4 & 16.0 & 7.0 & & 23.0 & \\
\hline \multirow{2}{*}{$\begin{array}{l}\text { Formal communication } \\
\text { mechanisms exist to } \\
\text { support user and IT } \\
\text { support staff } \\
\text { communication }\end{array}$} & $\mathrm{N}$ & 97 & 150 & 19 & 49 & 27 & \multirow[b]{2}{*}{2} & 76 & \multirow[b]{2}{*}{10} \\
\hline & $\%$ & 28.4 & 43.9 & 5.6 & 14.3 & 7.9 & & 22.2 & \\
\hline \multirow{2}{*}{$\begin{array}{l}\text { Adequate } \\
\text { communication } \\
\text { mechanisms exist to } \\
\text { support shared }\end{array}$} & $\mathrm{N}$ & 106 & 148 & 22 & 48 & 20 & \multirow[b]{2}{*}{2} & 68 & \multirow[b]{2}{*}{11} \\
\hline & $\%$ & 30.8 & 43.0 & 6.4 & 14.0 & 5.8 & & 19.8 & \\
\hline
\end{tabular}




\begin{tabular}{|c|c|c|c|c|c|c|c|c|c|}
\hline \multicolumn{10}{|l|}{$\begin{array}{l}\text { communication across } \\
\text { all organisational levels }\end{array}$} \\
\hline Staff have been included & $\mathrm{N}$ & 111 & 153 & 17 & 43 & 19 & \multirow[b]{2}{*}{2} & 62 & \multirow[b]{2}{*}{12} \\
\hline $\begin{array}{l}\text { in decision-making } \\
\text { processes }\end{array}$ & $\%$ & 32.4 & 44.6 & 5.0 & 12.5 & 5.5 & & 18 & \\
\hline \multirow{2}{*}{$\begin{array}{l}\text { Current work practices } \\
\text { are adequately } \\
\text { supported by existing } \\
\text { information systems }\end{array}$} & $\mathrm{N}$ & 115 & 154 & 23 & 32 & 21 & \multirow[b]{2}{*}{2} & 53 & \multirow[b]{2}{*}{13} \\
\hline & $\%$ & 33.3 & 44.6 & 6.7 & 9.3 & 6.1 & & 15.4 & \\
\hline
\end{tabular}

\subsubsection{Participant responses regarding endorsement of EHRS implementation}

In response to questions regarding whether or not they would endorse EHRS implementation in PHCs, the majority of participants $(339,97.7 \%)$ stated that they would endorse it, compared with eight (2.3\%) who said they would not (see Table 5.3.9). Four $(1.1 \%)$ participants did not declare whether or not they endorsed the implementation of an EHRS in PHCs.

Table 5.3.9: Do you endorse the implementation of an EHRS in PHCs?

\begin{tabular}{|c|c|c|}
\hline & Frequency & Percent \\
\hline Yes & 339 & 96.6 \\
\hline No & 8 & 2.3 \\
\hline Total & 347 & 98.9 \\
\hline
\end{tabular}

In response to the question of whether they would be resistant to having EHRS in the workplace, again, the majority $(318,91.6 \%)$ stated that they would not be resistant, whilst twenty-nine $(8.3 \%)$ stated that they would be resistant (see Table 5.3.10). Four (1.1\%) participants did not declare whether or not they would resist the implementation.

Table 5.3.10: Would you be resistant to an EHRS?

\begin{tabular}{|c|c|c|}
\hline & Frequency & Percent \\
\hline Yes & 29 & 8.3 \\
\hline No & 318 & 90.6 \\
\hline Total & 347 & 98.9 \\
\hline
\end{tabular}

The participants were asked why they would or would not be resistant to the implementation of an EHRS. The reasons given for not resisting EHRS implementation 
were related to it contributing to the system since it is "efficient", "helps people in their profession", "improves customer services", is "fast and accurate" and keeps "developing". Only one of the participants who said they would resist it provided a reason, stating that there was "no training and personnel".

\subsubsection{Open-ended question}

The responses to the open-ended question, "Do you have any recommendations for the decision-makers to improve the implementation of the EHRS?" A total of 232 responses were obtained from the participants, which were then categorised into seventeen themes. The percentage of responses varied from one theme to another (see Table 5.3.11). The most frequent recommendations were related to usability, which constituted $13 \%(n=29)$ of the recommendations. In this theme, the PHC staff suggested developing the infrastructure of the PHCs $11 \%(n=25)$, which includes implementing a high-speed Internet connection. In response to this open-ended question, PHC staff demanded an upgrade of the hardware in the PHCs. This demand appears twenty-three times (10\%). Nine percent $(n=21)$ of the recommendations are related to training. PHCs staff recommended providing adequate training programmes. Similar to training, recommendations to involve end users were raised twenty-one times (9\%). The least common recommendation was regarding awareness, which arose only three times (1\%). 
Table 5.3.11: Suggestions made by participants regarding recommendations and requirements for successful EHRS implementation.

\begin{tabular}{|c|c|c|c|c|}
\hline Rank & Themes & Example of responses & Frequency & Percentage \\
\hline 1 & Easy to use & $\begin{array}{c}\text { "Easy system" } \\
\text { "Usability is important" }\end{array}$ & 29 & $13 \%$ \\
\hline 2 & Infrastructure & $\begin{array}{l}\text { "Appropriate infrastructure" } \\
\text { "Fast Internet" } \\
\text { "Internet connection in the } \\
\text { PHCs" } \\
\text { "Infrastructure is very poor" }\end{array}$ & 25 & $11 \%$ \\
\hline 3 & Hardware & $\begin{array}{c}\text { "We need new computers" } \\
\text { "High performance computers } \\
\text { and printers" } \\
\text { "We ask them to change our } \\
\text { old computers" }\end{array}$ & 23 & $10 \%$ \\
\hline 4 & Training & $\begin{array}{l}\text { “Training, training, training” } \\
\text { “Adequate training must be } \\
\text { available for users" }\end{array}$ & 21 & $9 \%$ \\
\hline 5 & Staff involvement & $\begin{array}{l}\text { "Members of the centre should } \\
\text { represent us during the } \\
\text { implementation." } \\
\text { "They should hear our voice" }\end{array}$ & 22 & $9 \%$ \\
\hline 6 & Technical support & $\begin{array}{c}\text { "24-hours support” } \\
\text { "We need technical support all } \\
\text { the time", } \\
\text { "Technical support is } \\
\text { important" }\end{array}$ & 18 & $8 \%$ \\
\hline 7 & $\begin{array}{l}\text { Consider PHC } \\
\text { staff' } \\
\text { requirements }\end{array}$ & $\begin{array}{l}\text { "We have specific } \\
\text { requirements that should be } \\
\text { available in the EHRS" } \\
\text { "Take our requirements into } \\
\text { consideration" }\end{array}$ & 13 & $6 \%$ \\
\hline
\end{tabular}




\begin{tabular}{|c|c|c|c|c|}
\hline & & $\begin{array}{c}\text { "We will use the system and } \\
\text { we have some requirements } \\
\text { that must be available in the } \\
\text { system" }\end{array}$ & & \\
\hline 8 & $\begin{array}{l}\text { Keenness for } \\
\text { EHRS } \\
\text { implementation }\end{array}$ & $\begin{array}{c}\text { "I asked the Ministry to } \\
\text { implement the EHRS very } \\
\text { soon" } \\
\text { "We need it as soon as } \\
\text { possible" }\end{array}$ & 13 & $6 \%$ \\
\hline 9 & Secure system & $\begin{array}{l}\text { "Strong and secure system" } \\
\text { "They should use anti-virus } \\
\text { software to protect the system" } \\
\text { "The IT department must test } \\
\text { the system before } \\
\text { implementing it to make sure it } \\
\text { is protected against any } \\
\text { breaches" }\end{array}$ & 13 & $6 \%$ \\
\hline 10 & $\begin{array}{l}\text { Link all PHCs } \\
\text { together }\end{array}$ & $\begin{array}{c}\text { "It is better to link all PHCs } \\
\text { with each other" } \\
\text { "Information exchange } \\
\text { between all PHCs" }\end{array}$ & 11 & $5 \%$ \\
\hline 11 & Manpower & $\begin{array}{c}\text { "They must provide enough } \\
\text { users to work on the system" } \\
\text { "There is a shortage in } \\
\text { manpower" }\end{array}$ & 9 & $4 \%$ \\
\hline 12 & $\begin{array}{l}\text { Workflow } \\
\text { analysis and } \\
\text { redesign }\end{array}$ & $\begin{array}{c}\text { "PHC general managers must } \\
\text { consider business process } \\
\text { redesign" } \\
\text { "Centres redesigned before } \\
\text { the implementation" }\end{array}$ & 6 & $3 \%$ \\
\hline
\end{tabular}




\begin{tabular}{|c|c|c|c|c|}
\hline 13 & $\begin{array}{c}\text { Display in Arabic } \\
\text { and English }\end{array}$ & $\begin{array}{c}\text { "We need the system in two } \\
\text { languages, Arabic and } \\
\text { English" } \\
\text { "The system should be } \\
\text { available in Arabic for Saudi } \\
\text { users and English for non- } \\
\text { Saudi users" }\end{array}$ & 7 & $3 \%$ \\
\hline 14 & Efficient system & $\begin{array}{c}\text { "I need a highly efficient } \\
\text { system" } \\
\text { "Select an efficient system" }\end{array}$ & 8 & $3 \%$ \\
\hline 15 & $\begin{array}{l}\text { Link with } \\
\text { hospitals }\end{array}$ & $\begin{array}{c}\text { "I wish to link the centres with } \\
\text { hospitals electronically" } \\
\text { "We need to share patient } \\
\text { information with the } \\
\text { hospitals" }\end{array}$ & 7 & $3 \%$ \\
\hline 16 & $\begin{array}{l}\text { Store patient data } \\
\text { in cloud }\end{array}$ & $\begin{array}{l}\text { "I suggest uploading all } \\
\text { patient data to the cloud" } \\
\text { "It is better to save all data in } \\
\text { the cloud" }\end{array}$ & 4 & $2 \%$ \\
\hline 17 & Awareness & $\begin{array}{c}\text { "We need to be informed } \\
\text { about everything in the } \\
\text { implementation" }\end{array}$ & 3 & $1 \%$ \\
\hline & Total & & 232 & $100 \%$ \\
\hline
\end{tabular}




\subsection{Inferential statistics}

As illustrated in Chapters Two and Three, individual demographic and characteristics differences such as gender, age, and experience with PC influence the level of individual readiness to implement new EHRS (will be discussed in Chapter Eight). Therefore, a nonparametric test was performed to determine whether participants' demographic differences have any influence on individual readiness for EHRS implementation.

\section{Examining differences relating to gender}

A Mann-Witney $U$ test was performed on one variable in the questionnaire, participant gender. The male and female participants were not significantly different in terms of the benefits scale $(\mathrm{p}=0.506)$ and the readiness scale $(\mathrm{p}=0.344)$ (see Table 5.4.1).

Table 5.4.1: Mann-Witney U test for gender

\begin{tabular}{|c|c|c|c|c|}
\hline & $\begin{array}{l}\text { PHC staff awareness } \\
\text { about perceived } \\
\text { usefulness of an } \\
\text { EHRS }\end{array}$ & $\begin{array}{c}\text { Readiness } \\
\text { of PHCs }\end{array}$ & $\begin{array}{c}\text { PHC staff } \\
\text { resistance }\end{array}$ & $\begin{array}{c}\text { PHC staff } \\
\text { willingness }\end{array}$ \\
\hline $\begin{array}{c}\text { p. value } \\
\text { for } \\
\text { gender }\end{array}$ & .506 & .344 & .079 & .925 \\
\hline
\end{tabular}

\section{Examining differences relating to occupation}

A Kruskal-Wallis test showed no significant difference between participants in regard to their occupation when looking at perceived usefulness $(p=0.450)$, readiness $(p=0.475)$, resistance $(\mathrm{p}=0.441)$ and willingness $(\mathrm{p}=0.467)$ (see Table 5.4.2).

\section{Examining differences relating to gender}

A Kruskal-Wallis test showed no significant difference between age categories when looking at perceived usefulness $(\mathrm{p}=0.074)$, readiness $(\mathrm{p}=0.616)$, resistance $(\mathrm{p}=0.785)$ and willingness $(\mathrm{p}=0.055)$ (see Table 5.4.2). Moreover, age was examined using a MannWitney U test for two categories only (25 to 34 and 35 to 44), as the other age categories had only a small number of participants, which also showed no significant difference between the two age categories. 


\section{Examining differences relating to experience with using a personal computer}

Experience using computers was examined for its effect on participant perceptions of the benefits of an EHRS and their readiness to implement them. Using three categories of experience, a Kruskal-Wallis test showed no significant difference between the categories when looking at perceived usefulness $=(\mathrm{p}=0.757)$, readiness $(\mathrm{p}=0.925)$, resistance $(\mathrm{p}=0.409)$, and willingness $(\mathrm{p}=0.146)$ (see Table 5.4.2).

\section{Examining differences relating to years of job experience}

As seen in Table 5.4.2, experience in current position was also examined for its effect on the participants' perceptions of the benefits of EHRS and their readiness to implement them. A Kruskal Wallis test showed no significant difference between the four categories of experience when looking at benefits $(\mathrm{p}=0.771)$, readiness $(\mathrm{p}=0.984)$, resistance $(\mathrm{p}=0$. $663)$ and willingness $(\mathrm{p}=0.529)$.

\section{Examining differences relating to province}

A Kruskal-Wallis test was conducted to measure the differences between the regions in Saudi Arabia. No significant difference to perceived usefulness was found between participants in regard to their region $(\mathrm{p}=0.283)$, readiness $(\mathrm{p}=0.853)$, resistance $(\mathrm{p}=0.890)$, or willingness $(\mathrm{p}=0.145)$ (see Table 5.4.2).

Table 5.4.2: Kruskal-Wallis Test for occupation, age, computer experience, experience at current position, and province differences.

\begin{tabular}{|c|c|c|c|c|}
\hline & $\begin{array}{c}\text { PHC staff } \\
\text { awareness of the } \\
\text { usefulness of } \\
\text { EHRS }\end{array}$ & $\begin{array}{c}\text { Readiness } \\
\text { of PHCs }\end{array}$ & $\begin{array}{l}\text { PHC staff } \\
\text { resistance }\end{array}$ & $\begin{array}{l}\text { PHC staff } \\
\text { willingness }\end{array}$ \\
\hline $\begin{array}{l}\text { P. value for } \\
\text { occupation }\end{array}$ & .450 & .475 & .441 & .467 \\
\hline P. value for age & .074 & .925 & .785 & .055 \\
\hline $\begin{array}{l}P . \text { value for } \\
\text { experience with } \\
P C\end{array}$ & .757 & .968 & .409 & .146 \\
\hline $\begin{array}{l}P . \text { value for } \\
\text { experience in } \\
\text { current position }\end{array}$ & .771 & .984 & .663 & .529 \\
\hline $\begin{array}{l}\text { P. value for } \\
\text { province }\end{array}$ & .283 & .853 & .890 & .145 \\
\hline
\end{tabular}




\subsection{Summary}

The aim of this study was to assess PHC readiness for EHRS implementation from a potential user perspective. Similar to Study One, the majority of participants were male (74.4\%), were nurses (29.6\%), and aged between twenty-five and forty-four years (89.7\%). When assessing PHC staff awareness of the usefulness of EHRS, responses were mostly positive. The majority of the participants acknowledged the benefits of EHRS based on the high agreement across the benefits scale items (ranging between $90.5 \%$ and $84.2 \%$ agreement).

As for PHC readiness, the outcomes were mixed. Whilst the participants were positive about their readiness to implement EHRS in certain areas, there remained a number of challenges that they perceived, such as PHC staff involvement in the implementation procedures or the establishment of proper communication mechanisms between project team and PHCs that would prevent them from being fully ready. Finally, when asking the participants whether or not they would endorse EHRS in PHCs, 97.7\% stated that they would do so. The results, however, show poor readiness at the organisational level, with training being unavailable during the pre-implementation phase, whereas $63.7 \%$ provided negative responses about the availability of adequate training. In addition, $69.2 \%$ of the participants disagreed when asked if champions are available to support the implementation. Moreover, $\mathrm{PHC}$ readiness was found to be poor at the technological level due to weak infrastructure and a lack of appropriate hardware, which can be inferred form the participants claims in their responses to the open-ended question. The following chapter presents the findings related to the implemented EHRS in Saudi PHCs obtained from EHRS end-users via electronic questionnaire-based research. 
6 Chapter Six: Study Three: Evaluation of the Implemented EHRS in PHCs in SA from the Perspective of Endusers 


\subsection{Introduction}

This chapter presents the results and statistics from a study aimed at evaluating the EHRS implemented in PHCs in SA, and also to determine the main facilitators and barriers to EHRS implementation from an end-user perspective. To achieve the above aims, three different statistical tests were performed. Firstly, descriptive statistics to determine the overall attitudes regarding the implementation of the EHRS, as well as the major and minor barriers to EHRS implementation. This test assists in displaying all obtained data and then predicting which items in the questionnaire are more important and which factors recorded more satisfaction than others. Secondly, inferential statistics using differences and correlation tests were performed to test the study hypotheses (see Chapter Three). The items in the questionnaire have been categorised into five different scales, as follows: perceived usefulness of the EHRS, positive attitude, negative attitude, training and support, and barriers. An additional two open-ended questions were included to identify any further facilitators and barriers to EHRS implementation other than those included in previous scales.

\subsection{Scale reliability:}

This study utilised five scales (themes). This section measures the reliability of each of those scales (perceived usefulness of EHRS, positive attitude, training and support, negative attitude, and barriers). As can be seen in Table 6.2.1, the reliability of all scales is high. The advantages scale shows the highest reliability (0.92), followed by satisfaction (0.89), positive attitude (0.88), barriers (0.83) and, lastly, negative attitude (0.70).

Table 6.2.1: Scale reliability using Cronbach's Alpha

\begin{tabular}{|l|c|c|}
\hline Scale & Number of Items & Cronbach's Alpha \\
\hline Perceived usefulness of the EHRS & $\mathbf{1 7}$ & $\mathbf{0 . 9 2}$ \\
\hline Positive attitude & 15 & 0.89 \\
\hline Training and support & 7 & 0.90 \\
\hline Negative attitude & 7 & 0.70 \\
\hline Barriers & $\mathbf{1 6}$ & $\mathbf{0 . 8 3}$ \\
\hline Entire questionnaire & $\mathbf{6 2}$ & $\mathbf{0 . 8 3}$ \\
\hline
\end{tabular}




\subsection{Descriptive statistics of participants responses}

In order to examine the implemented EHRS in Saudi PHCs and also to determine the main barriers to implementation, descriptive statistics were performed to display the data obtained by the participants. Out of the 483 healthcare practitioners working in the selected PHCs, 205 completed the questionnaire. This indicates a response rate of $42.4 \%$. The highest number of responses were received from the capital, Riyadh $(n=60 ; 29.3 \%)$, followed by Gassim $(n=52 ; 25.4 \%)$, Makkah $(n=42 ; 20.5 \%)$, Aljouf $(n=33 ; 16.1 \%)$ and Albaha $(\mathrm{n}=18 ; 8.8 \%)$ (see Table 6.3.1). There were 159 male participants $(77.6 \%)$ and forty-six female participants $(22.4 \%)$.

Table 6.3.1: Participant distribution based on geographical location

\begin{tabular}{|l|c|c|}
\hline Region & Frequency & Percent \\
\hline Riyadh & 60 & 29.3 \\
\hline Gassim & 52 & 25.4 \\
\hline Aljouf & 33 & 16.1 \\
\hline Albaha & 18 & 8.8 \\
\hline Makkah & 42 & 20.5 \\
\hline Total & 205 & 100.0 \\
\hline
\end{tabular}

Participants were asked to specify their gender. As shown in Table 6.3.2, the participants were mostly male $(n=159 ; 77.6 \%)$. Out of 205 participants, only forty-six females responded to the questionnaire.

Table 6.3.2: Participant distribution based on gender

\begin{tabular}{|l|c|c|}
\hline Gender & Frequency & Percent \\
\hline Female & 46 & 22.4 \\
\hline Male & 159 & 77.6 \\
\hline Total & 205 & 100.0 \\
\hline
\end{tabular}

The participants had a range of occupations within the healthcare field. The highest proportion were nurses $(\mathrm{n}=62 ; 32 \%)$, followed by administrators $(\mathrm{n}=54 ; 26.4 \%)$, physicians $(n=24 ; 12.4 \%)$ and pharmacists $(n=20 ; 10.3 \%)$. Table 6.3 .3 shows the full range of occupations of the participants. Eleven (5.4\%) participants did not declare their occupation. 
Table 6.3.3: Participant distribution based on occupation

\begin{tabular}{|l|c|c|}
\hline Occupation & Frequency & Percent \\
\hline Physician & 24 & 11.7 \\
\hline Administrator & 54 & 26.4 \\
\hline Nurse & 62 & 30.2 \\
\hline Lap Technician & 12 & 5.9 \\
\hline Pharmacist & 20 & 9.8 \\
\hline Radiologist & 9 & 4.4 \\
\hline Dentist & 13 & 6.3 \\
\hline Total & 194 & 94.6 \\
\hline
\end{tabular}

As outlined in Table 6.3.4, the majority of the participants $(n=113 ; 55.1 \%)$ were between twenty-five and thirty-four years of age.

Table 6.3.4: Distribution of participants based on age

\begin{tabular}{|l|c|c|}
\hline Age & Frequency & Percent \\
\hline 18 to 24 & 1 & .5 \\
\hline 25 to 34 & 113 & 55.1 \\
\hline 35 to 44 & 68 & 33.2 \\
\hline 45 to 54 & 19 & 9.3 \\
\hline 55 to 64 & 2 & 1.0 \\
\hline 65 to 74 & 2 & 1.0 \\
\hline Total & 205 & 100.0 \\
\hline
\end{tabular}

In terms of the time the participants had spent in their roles, sixty-one (29.8\%) had been in their position between one and five years, fifty-eight (28.3\%) between six and ten years, and fifty-six (27.3\%) between eleven and fifteen years. Only twenty $(9.8 \%)$ participants had been in their position more than fifteen years, and only ten (4.9\%) for less than one year (see Table 6.3.5).

Table 6.3.5: Participant distribution based on experience in their present position

\begin{tabular}{|l|c|c|}
\hline Length of experience & Frequency & Percent \\
\hline Less than 1 year & 10 & 4.9 \\
\hline 1 to 5 years & 61 & 29.8 \\
\hline 6 to 10 years & 58 & 28.3 \\
\hline 11 - 15 years & 56 & 27.3 \\
\hline More than 15 years & 20 & 9.8 \\
\hline Total & 205 & 100.0 \\
\hline
\end{tabular}


In response to the question regarding experience with using a personal computer at home, it was evident that the majority had more than five years' experience. See Table 6.3.6 for details.

Table 6.3.6: Participant distribution based on their experience with using a personal computer

\begin{tabular}{|l|c|c|}
\hline Length of experience & Frequency & Percent \\
\hline Less than 1 year & 5 & 2.4 \\
\hline 1 to 5 years & 22 & 10.7 \\
\hline 6 to 10 years & 52 & 25.4 \\
\hline 11 - 15 years & 81 & 39.5 \\
\hline More than 15 years & 45 & 22.0 \\
\hline Total & 205 & 100.0 \\
\hline
\end{tabular}

In terms of using an EHRS, the survey identified that 118 (58.4\%) of the participants had less than one year of experience. In contrast, sixty-two (30.7\%) participants had between 1 and 5 years' experience, eighteen (8.9\%) stated that they had between six and ten years' experience (18), and only four (2\%) participants had between eleven and fifteen years' experience (4) (see Table 6.3.7). Three (1.5\%) participants did not declare their experience with using an EHRS.

Table 6.3.7 Participant distribution based on their experience with using an EHRS

\begin{tabular}{|l|c|c|}
\hline Length of experience & Frequency & Percent \\
\hline Less than 1 year & 118 & 57.6 \\
\hline 1 to 5 years & 62 & 30.2 \\
\hline 6 to 10 years & 18 & 8.8 \\
\hline 11 - 15 years & 4 & 2.0 \\
\hline Total & 202 & 98.5 \\
\hline
\end{tabular}

When asked about whether they were still using the EHRS in their workplace, the majority $(n=157 ; 76.6 \%)$ of the participants stated that they did not use the EHRS at their workplace, with only forty-six $(22.4 \%)$ using the EHRS at their workplace. Two (1\%) participants did not declare whether or not they are still using the EHRS at their workplace (see Table 6.3.8). 
Table 6.3.8: Participant distribution based on current usage of the EHRS in the workplace

\begin{tabular}{|l|c|c|}
\hline EHRS used? & Frequency & Percent \\
\hline Yes & 46 & 22.4 \\
\hline No & 157 & 76.6 \\
\hline Total & 203 & 99.0 \\
\hline
\end{tabular}

\subsubsection{Overall attitudes towards the implementation of EHRS}

\section{Perceived usefulness of the EHRS}

The participants expressed a very high level of satisfaction with the usefulness of an EHRS. It was evident from the participants' responses across all the items that they were satisfied with the usefulness of the implemented EHRS. Based on the questions in the second section of the questionnaire, relating to the benefits of using the EHRS, such as medication error reduction, cost reduction, improved patient safety and quality of care, there was a high level of agreement with all items, ranging from a high of $93.7 \%$ to a low of $87.3 \%$. Table 6.3 .9 shows that the items with the highest level of endorsement were: 1) "EHRS reduces costs through decreased paperwork, improved safety, reduced duplication of testing and improved health" (93.6\%); 2)“EHRS help to promote legible documents" (93.1\%); 3) "Sharing electronic information with patients and other clinicians is more secure when using the EHR system" (92.7\%); and, 4), "The EHR system helps to do streamlined coding" (92.7\%). Those items, with a lower level of endorsement, were: 14) "Using the EHR system helps to provide safer care" (88.7\%); and 15) "Information from the EHRS enables me to make better decisions about patient care" (88.3\%); 16) "Using the EHR system helps to effectively diagnose patients" (88.2\%) and; 17) "Using the EHR system helps to reduce medical errors" (87.3\%). 
Table 6.3.9: Degree of endorsement for each of the seventeen questions relating to perceived usefulness of EHRS

\begin{tabular}{|c|c|c|c|c|c|c|c|c|c|}
\hline 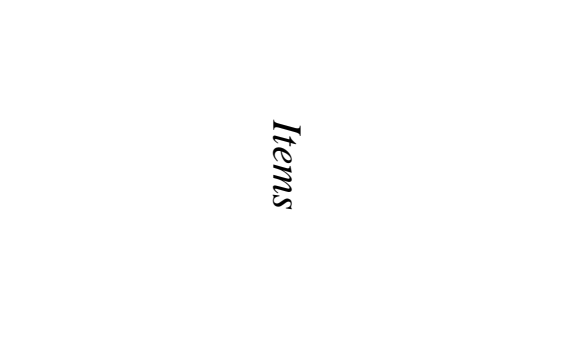 & & 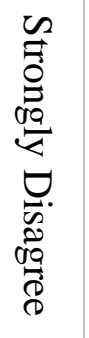 & 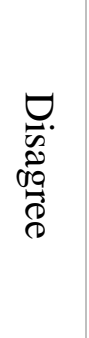 & 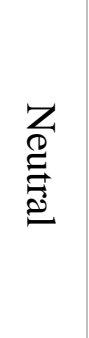 & $\underset{\substack{\infty \\
\mathbb{d}}}{\stackrel{D}{0}}$ & 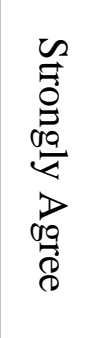 & 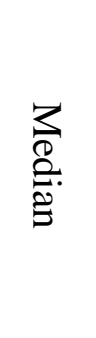 & $\begin{array}{l}\overrightarrow{0} \\
\stackrel{\overrightarrow{0}}{0} \\
00 \\
00 \\
0 \\
0 \\
0 \\
0 \\
0\end{array}$ & 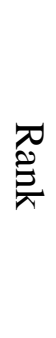 \\
\hline \multirow{2}{*}{$\begin{array}{l}\text { EHRS reduces costs through } \\
\text { decreased paperwork, improved } \\
\text { safety, reduced duplication of } \\
\text { testing and improved } \\
\text { healthcare }\end{array}$} & $\mathrm{N}$ & 5 & 4 & 4 & 87 & 105 & 5.00 & 192 & \\
\hline & $\%$ & 2.4 & 2.0 & 2.0 & 42.4 & 51.2 & & 93.6 & 1 \\
\hline \multirow{2}{*}{$\begin{array}{l}\text { EHRS help to promote legible } \\
\text { documents }\end{array}$} & $\mathrm{N}$ & 4 & 6 & 4 & 94 & 95 & 4.00 & 189 & \multirow{2}{*}{2} \\
\hline & $\%$ & 2.0 & 3.0 & 2.0 & 46.3 & 46.8 & & 93.1 & \\
\hline \multirow{2}{*}{$\begin{array}{l}\text { Sharing electronic information } \\
\text { with patients and other } \\
\text { clinicians is more secure when } \\
\text { using the EHR system }\end{array}$} & $\mathrm{N}$ & 6 & 4 & 5 & 91 & 99 & 4.00 & 190 & \multirow[b]{2}{*}{3} \\
\hline & $\%$ & 2.9 & 2.0 & 2.4 & 44.4 & 48.3 & & 92.7 & \\
\hline \multirow{2}{*}{$\begin{array}{l}\text { The EHR system helps with } \\
\text { streamlined coding }\end{array}$} & $\mathrm{N}$ & 4 & 6 & 5 & 97 & 93 & 4.00 & 190 & \multirow{2}{*}{4} \\
\hline & $\%$ & 2.0 & 2.9 & 2.4 & 47.3 & 45.4 & & 92.7 & \\
\hline \multirow{2}{*}{$\begin{array}{l}\text { EHRS enable quick access to } \\
\text { patient records for more } \\
\text { coordinated and efficient care. }\end{array}$} & $\mathrm{N}$ & 8 & 5 & 3 & 95 & 94 & 4.00 & 189 & \multirow[b]{2}{*}{5} \\
\hline & $\%$ & 3.9 & 2.4 & 1.5 & 46.3 & 45.9 & & 92.2 & \\
\hline \multirow{2}{*}{$\begin{array}{l}\text { Using the EHR system } \\
\text { improves patient and } \\
\text { healthcare } \\
\text { professionals' interaction and } \\
\text { communication as well as } \\
\text { healthcare convenience }\end{array}$} & $\mathrm{N}$ & 5 & 5 & 6 & 101 & 88 & 4.00 & 189 & \multirow[b]{2}{*}{6} \\
\hline & $\%$ & 2.4 & 2.4 & 2.9 & 49.3 & 42.9 & & 92.2 & \\
\hline \multirow{2}{*}{$\begin{array}{l}\text { The EHR system allows me to } \\
\text { spend more time on other } \\
\text { aspects of patient care }\end{array}$} & $\mathrm{N}$ & 6 & 6 & 5 & 94 & 94 & 4.00 & 188 & \multirow[b]{2}{*}{7} \\
\hline & $\%$ & 2.9 & 2.9 & 2.4 & 45.9 & 45.9 & & 91.8 & \\
\hline \multirow{2}{*}{$\begin{array}{l}\text { EHRS help to provide accurate } \\
\text { information }\end{array}$} & $\mathrm{N}$ & 5 & 7 & 5 & 100 & 88 & 4.00 & 188 & \multirow{2}{*}{8} \\
\hline & $\%$ & 2.4 & 3.4 & 2.4 & 48.8 & 42.9 & & 91.7 & \\
\hline \multirow{2}{*}{$\begin{array}{l}\text { EHRS enable safer and more } \\
\text { reliable prescribing }\end{array}$} & $\mathrm{N}$ & 4 & 6 & 8 & 88 & 99 & 4.00 & 187 & \multirow{2}{*}{9} \\
\hline & $\%$ & 2.0 & 2.9 & 3.9 & 42.9 & 48.3 & & 91.2 & \\
\hline \multirow{2}{*}{$\begin{array}{l}\text { EHRS help to have complete } \\
\text { documentation }\end{array}$} & $\mathrm{N}$ & 6 & 8 & 6 & 92 & 93 & 4.00 & 185 & \multirow{2}{*}{10} \\
\hline & $\%$ & 2.9 & 3.9 & 2.9 & 44.9 & 45.4 & & 90.3 & \\
\hline \multirow{2}{*}{$\begin{array}{l}\text { EHRS provide accurate, up-to- } \\
\text { date and complete information } \\
\text { about patients at the point of } \\
\text { care }\end{array}$} & $\mathrm{N}$ & 5 & 8 & 7 & 106 & 79 & 4.00 & 185 & \multirow[b]{2}{*}{11} \\
\hline & $\%$ & 2.4 & 3.9 & 3.4 & 51.7 & 38.5 & & 90.2 & \\
\hline \multirow{3}{*}{$\begin{array}{l}\text { EHRS improve end- } \\
\text { user productivity and efficiency }\end{array}$} & $\mathrm{N}$ & 6 & 9 & 7 & 90 & 92 & 4.00 & 182 & \multirow{2}{*}{12} \\
\hline & $\%$ & 2.9 & 4.4 & 3.4 & 44.1 & 45.1 & & 89.2 & \\
\hline & $\mathrm{N}$ & 6 & 6 & 11 & 88 & 93 & 4.00 & 181 & 13 \\
\hline
\end{tabular}




\begin{tabular}{|c|c|c|c|c|c|c|c|c|c|}
\hline $\begin{array}{l}\text { EHRS improve the privacy and } \\
\text { security of patient data }\end{array}$ & $\%$ & 2.9 & 2.9 & 5.4 & 43.1 & 45.6 & & 88.7 & \\
\hline \multirow{2}{*}{$\begin{array}{l}\text { Using the EHR system helps } \\
\text { to provide safer care }\end{array}$} & $\mathrm{N}$ & 4 & 8 & 11 & 93 & 88 & 4.00 & 181 & \multirow{2}{*}{14} \\
\hline & $\%$ & 2.0 & 3.9 & 5.4 & 45.6 & 43.1 & & 88.7 & \\
\hline \multirow{2}{*}{$\begin{array}{l}\text { Information from the EHR } \\
\text { system enables me to make } \\
\text { better decisions about patient } \\
\text { care }\end{array}$} & $\mathrm{N}$ & 5 & 8 & 11 & 99 & 82 & 4.00 & 181 & \multirow[b]{2}{*}{15} \\
\hline & $\%$ & 2.4 & 3.9 & 5.4 & 48.3 & 40.0 & & 88.3 & \\
\hline \multirow{2}{*}{$\begin{array}{l}\text { Using the EHR system helps } \\
\text { to effectively diagnose patients }\end{array}$} & $\mathrm{N}$ & 5 & 9 & 10 & 96 & 83 & 4.00 & 179 & \multirow{2}{*}{16} \\
\hline & $\%$ & 2.5 & 4.4 & 4.9 & 47.3 & 40.9 & & 88.2 & \\
\hline \multirow{2}{*}{$\begin{array}{l}\text { Using the EHR system helps } \\
\text { to reduce medical errors }\end{array}$} & $\mathrm{N}$ & 6 & 7 & 13 & 96 & 83 & 4.00 & 179 & \multirow{2}{*}{17} \\
\hline & $\%$ & 2.9 & 3.4 & 6.3 & 46.8 & 40.5 & & 87.3 & \\
\hline
\end{tabular}

\section{Positive Attitudes towards use of the EHRS}

Based on the responses to the fourteen items representing positive attitudes toward EHRS implementation and use, it was clear that there was a high level of positive endorsement. The highest level of endorsement was $97.5 \%$, and the lowest $79.6 \%$. Looking at the items individually, those with the highest level of endorsement were 1) "Overall, I prefer using the EHR system to the paper-based system" (97.5\%); 2) "The EHR system is more efficient than a paper-based system" (95.1\%); and 3) "Using EHRS leads to better adherence to policies and procedures" (92.6\%). The items with the lowest level of endorsement (but still having more agreement than disagreement) were: 13) "The EHR system takes into account the specific needs of my care area $(s)$ " $(81.9 \%)$; 14) "Overall, the introduction of the EHR system has been effective" (80\%) and; 15) "I'm committed to the successful use of the EHR system" (79.6\%).

Moreover, a positive attitude scale provided participant responses to examined factors such as EHRS usability, efficiency and information quality. The table below shows a high level of agreement towards all items representing EHRS usability: "The EHR system is easy to use" (86.2\%); "I am physically comfortable while using the EHR system equipment and hardware" (87.2\%). Furthermore, a high level of agreement was also recoded with items representing EHRS efficiency: "The EHR system is more efficient than a paper-based system" (95.1\%). Table 6.3.10, also illustrated the level of agreement towards information quality items. High agreement was also found with "I can depend on the accuracy of the EHR system" (93.6\%); and "Information almost never gets lost in the EHR system" (83.3\%). 
Table 6.3.10: Responses and endorsement to fourteen statements on positive attitudes toward the use of the EHRS scale

\begin{tabular}{|c|c|c|c|c|c|c|c|c|c|}
\hline$\underset{\overparen{\Xi}}{\widetilde{\Xi}}$ & & 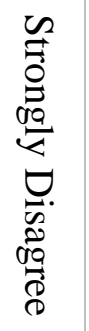 & 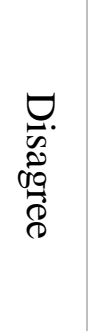 & 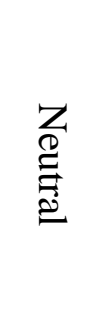 & 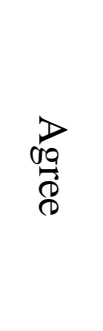 & 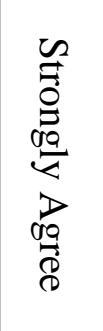 & 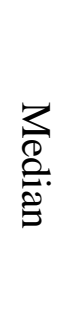 & 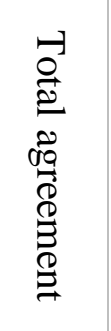 & 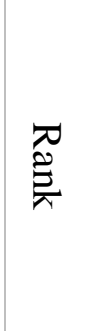 \\
\hline \multirow{2}{*}{$\begin{array}{l}\text { Overall, I prefer using the } E H R \\
\text { system to the paper-based } \\
\text { system }\end{array}$} & $\mathrm{N}$ & 1 & 2 & 2 & 91 & 107 & \multirow[b]{2}{*}{4} & 198 & \multirow[b]{2}{*}{1} \\
\hline & $\%$ & .5 & 1.0 & 1.0 & 44.8 & 52.7 & & 97.5 & \\
\hline \multirow{2}{*}{$\begin{array}{l}\text { The EHR system is more } \\
\text { efficient than a paper-based } \\
\text { system }\end{array}$} & $\mathrm{N}$ & & 4 & 6 & 80 & 113 & \multirow[b]{2}{*}{4} & 193 & \multirow{2}{*}{2} \\
\hline & $\%$ & & 2.0 & 3.0 & 39.4 & 55.7 & & 95.1 & \\
\hline \multirow{2}{*}{$\begin{array}{l}\text { I can depend on the accuracy of } \\
\text { the EHR system }\end{array}$} & $\mathrm{N}$ & 1 & 6 & 6 & 104 & 85 & \multirow{2}{*}{4} & 189 & \multirow{2}{*}{3} \\
\hline & $\%$ & .5 & 3.0 & 3.0 & 51.5 & 42.1 & & 93.6 & \\
\hline \multirow{2}{*}{$\begin{array}{l}\text { Using EHRS leads to better } \\
\text { adherence to policies and } \\
\text { procedures }\end{array}$} & $\mathrm{N}$ & 3 & 5 & 7 & 108 & 81 & \multirow[b]{2}{*}{4} & 189 & \multirow[b]{2}{*}{4} \\
\hline & $\%$ & 1.5 & 2.5 & 3.4 & 52.9 & 39.7 & & 92.6 & \\
\hline \multirow{2}{*}{$\begin{array}{l}\text { The EHR system facilitates the } \\
\text { communication of patient } \\
\text { information among members of } \\
\text { our healthcare team. }\end{array}$} & $\mathrm{N}$ & 2 & 4 & 13 & 87 & 99 & \multirow[b]{2}{*}{4} & 186 & \multirow[b]{2}{*}{5} \\
\hline & $\%$ & 1.0 & 2.0 & 6.3 & 42.4 & 48.3 & & 90.7 & \\
\hline \multirow{2}{*}{$\begin{array}{l}\text { I am physically comfortable } \\
\text { while using the EHR system } \\
\text { equipment and hardware }\end{array}$} & $\mathrm{N}$ & 4 & 8 & 14 & 90 & 88 & \multirow[b]{2}{*}{4} & 178 & \\
\hline & $\%$ & 2.0 & 3.9 & 6.9 & 44.1 & 43.1 & & 87.2 & \\
\hline \multirow{2}{*}{$\begin{array}{l}\text { The EHR system has improved } \\
\text { my practice }\end{array}$} & $\mathrm{N}$ & 4 & 6 & 21 & 78 & 96 & \multirow{2}{*}{4} & 174 & \multirow{2}{*}{7} \\
\hline & $\%$ & 2.0 & 2.9 & 10.2 & 38.0 & 46.8 & & 84.8 & \\
\hline \multirow{2}{*}{$\begin{array}{l}\text { I feel the use of the EHR system } \\
\text { has improved the quality of } \\
\text { patient care }\end{array}$} & $\mathrm{N}$ & 2 & 7 & 21 & 99 & 75 & \multirow[b]{2}{*}{4} & 174 & \multirow[b]{2}{*}{8} \\
\hline & $\%$ & 1.0 & 3.4 & 10.3 & 48.5 & 36.8 & & 85.3 & \\
\hline \multirow{2}{*}{ The EHR system is easy to use } & $\mathrm{N}$ & 3 & 7 & 18 & 104 & 70 & \multirow{2}{*}{4} & 174 & \multirow{2}{*}{9} \\
\hline & $\%$ & 1.5 & 3.5 & 8.9 & 51.5 & 34.7 & & 86.2 & \\
\hline \multirow{2}{*}{$\begin{array}{l}\text { I feel the use of the system has } \\
\text { improved patient care outcomes }\end{array}$} & $\mathrm{N}$ & 5 & 5 & 25 & 84 & 86 & \multirow{2}{*}{4} & 170 & \multirow{2}{*}{10} \\
\hline & $\%$ & 2.4 & 2.4 & 12.2 & 41.0 & 42.0 & & 83.0 & \\
\hline Information almost never gets & $\mathrm{N}$ & 5 & 11 & 18 & 84 & 85 & 4 & 169 & 11 \\
\hline lost in the EHR system & $\%$ & 2.5 & 5.4 & 8.9 & 41.4 & 41.9 & 4 & 83.3 & 11 \\
\hline The EHR system takes into & $\mathrm{N}$ & 6 & 10 & 21 & 90 & 78 & & 168 & \\
\hline $\begin{array}{l}\text { account the specific needs of my } \\
\text { care area }(s)\end{array}$ & $\%$ & 2.9 & 4.9 & 10.2 & 43.9 & 38.0 & 4 & 81.9 & 12 \\
\hline Overall, the introduction of the & $\mathrm{N}$ & 8 & 9 & 24 & 79 & 85 & 4 & 164 & \\
\hline EHR system has been effective & $\%$ & 3.9 & 4.4 & 11.7 & 38.5 & 41.5 & 4 & 80.0 & \\
\hline I am committed to the & $\mathrm{N}$ & 12 & 7 & 23 & 86 & 77 & & 163 & \\
\hline $\begin{array}{l}\text { successful use of the EHR } \\
\text { system }\end{array}$ & $\%$ & 5.9 & 3.4 & 11.2 & 42.0 & 37.6 & 4 & 79.6 & \\
\hline
\end{tabular}




\section{Attitudes toward training and support}

Based on responses to the eight items representing training and support in Table 4.3.11, items with the highest level of endorsement were: 1) "I don't get as much help as I need to fix problems with EHRS" (72.1\%); and 2) "I am satisfied with the mechanism for making suggestions to improve the system" (16.6\%). The responses that received less satisfaction toward training were: 6) "Adequate resources were available when I was learning to use the EHR system" (11.7\%); and 8) "There was a campaign to introduce the EHR system prior to the implementation" (8.8\%). Overall, these findings indicate that participants were unsatisfied about the training and support provided to them.

Table 6.3.11: Participant responses to eight items reflecting attitudes toward training and support for EHRS

\begin{tabular}{|c|c|c|c|c|c|c|c|c|c|}
\hline$\underset{\overparen{\Xi}}{\widetilde{\Xi}}$ & & 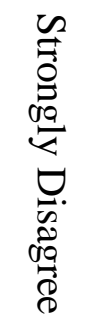 & 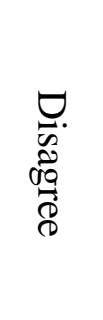 & 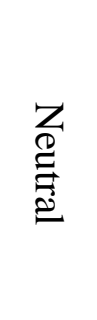 & 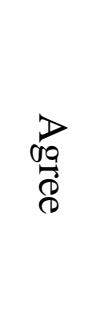 & 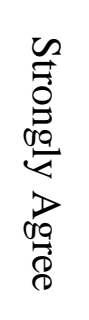 & : & 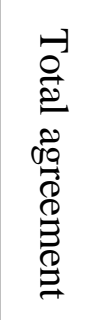 & 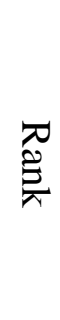 \\
\hline \multirow{2}{*}{$\begin{array}{l}\text { I don't get as much help as I } \\
\text { need to fix problems with } \\
\text { EHRS }\end{array}$} & $\mathrm{N}$ & 14 & 19 & 24 & 75 & 72 & \multirow{2}{*}{4} & 147 & \multirow{2}{*}{1} \\
\hline & $\%$ & 6.9 & 9.3 & 11.8 & 36.8 & 35.3 & & 72.1 & \\
\hline \multirow{2}{*}{$\begin{array}{l}\text { I am satisfied with the } \\
\text { mechanism for making } \\
\text { suggestions to improve the } \\
\text { system }\end{array}$} & $\mathrm{N}$ & 94 & 44 & 33 & 16 & 18 & \multirow[b]{2}{*}{2} & 34 & \multirow[b]{2}{*}{2} \\
\hline & $\%$ & 45.9 & 21.5 & 16.1 & 7.8 & 8.8 & & 16.6 & \\
\hline \multirow{2}{*}{$\begin{array}{l}\text { The training I received was } \\
\text { adequate }\end{array}$} & $\mathrm{N}$ & 76 & 70 & 26 & 23 & 10 & \multirow{2}{*}{2} & 33 & \multirow{2}{*}{3} \\
\hline & $\%$ & 37.1 & 34.1 & 12.7 & 11.2 & 4.9 & & 16.1 & \\
\hline \multirow{2}{*}{$\begin{array}{l}\text { I am satisfied with the } \\
\text { mechanism for } \\
\text { identifying/reporting issues } \\
\text { with the system }\end{array}$} & $\mathrm{N}$ & 95 & 50 & 32 & 14 & 14 & \multirow[b]{2}{*}{2} & 28 & \multirow[b]{2}{*}{4} \\
\hline & $\%$ & 46.3 & 24.4 & 15.6 & 6.8 & 6.8 & & 13.6 & \\
\hline \multirow{2}{*}{$\begin{array}{l}\text { When the EHR system is } \\
\text { down, the backup methods } \\
\text { work adequately }\end{array}$} & $\mathrm{N}$ & 90 & 58 & 30 & 17 & 9 & \multirow[b]{2}{*}{2} & 26 & \multirow[b]{2}{*}{5} \\
\hline & $\%$ & 44.1 & 28.4 & 14.7 & 8.3 & 4.4 & & 12.7 & \\
\hline \multirow{2}{*}{$\begin{array}{l}\text { Adequate resources were } \\
\text { available when I was learning } \\
\text { to use the EHR system }\end{array}$} & $\mathrm{N}$ & 76 & 82 & 23 & 17 & 7 & \multirow[b]{2}{*}{2} & 24.0 & \multirow[b]{2}{*}{6} \\
\hline & $\%$ & 37.1 & 40.0 & 11.2 & 8.3 & 3.4 & & 11.7 & \\
\hline \multirow{3}{*}{$\begin{array}{l}\text { When I report problems with } \\
\text { the system that need fixing, I } \\
\text { receive adequate feedback }\end{array}$} & $\mathrm{N}$ & 109 & 51 & 25 & 8 & 12 & \multirow[b]{2}{*}{1} & 20.0 & \multirow[b]{2}{*}{7} \\
\hline & $\%$ & 53.2 & 24.9 & 12.2 & 3.9 & 5.9 & & 9.8 & \\
\hline & $\mathrm{N}$ & 89 & 75 & 23 & 11 & 7 & 2 & 18 & 8 \\
\hline
\end{tabular}




\section{Negative attitudes toward the EHRS:}

In this table, 'strongly agree' means that participants were dissatisfied with the scored item, while 'strongly disagree' reflects the participant satisfaction with that item (see Table 6.3.12). The proportion of neutral or disagreement is greater than the positive. This table contains items representing factors that influence EHRS implementation, such as user involvement and system efficiency, based on the responses to seven questions on the negative attitude scale. The negative attitude scale provides participant responses to items representing factors that influence EHRS implementation such as EHRS end-user involvement (see Chapter Two). Therefore, the highest level of endorsement was generated for user involvement: 1) "End-users should have been considered in the system design" (85.8\%). The second level of agreement was generated for 2) "It takes too much time to help others who don't know how to use the system" (63.4\%). However, lower agreement was generated for items: 5) "Using EHRS raises stress levels among practitioners" (12.8\%); 6) "The system makes me feel like I am no longer functioning as part of a team" (12.3\%); and 7) "The EHR system is considered to be an extra load at work" $(9.7 \%)$. 
Table 6.3.12: Participant responses to seven items reflecting negative attitudes toward the EHRS implemented in PHCs.

\begin{tabular}{|c|c|c|c|c|c|c|c|c|c|}
\hline$\underset{\overparen{3}}{\overparen{3}}$ & & 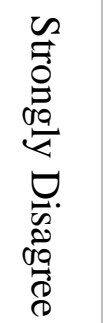 & 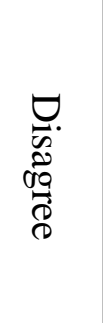 & 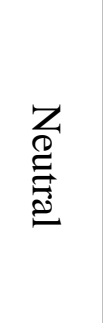 & $\underset{\substack{D \\
0}}{\stackrel{D}{8}}$ & 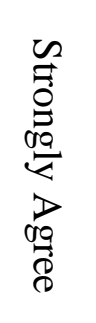 & . & 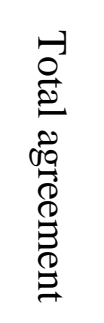 & $\begin{array}{l}\text { त्र } \\
\text { 突 }\end{array}$ \\
\hline \multirow{2}{*}{$\begin{array}{l}\text { End-users should have been } \\
\text { considered in the system } \\
\text { design }\end{array}$} & $\mathrm{N}$ & 2 & 6 & 21 & 56 & 120 & \multirow{2}{*}{5} & 176 & \multirow[b]{2}{*}{1} \\
\hline & $\%$ & 1.0 & 2.9 & 10.2 & 27.3 & 58.5 & & 85.8 & \\
\hline \multirow{2}{*}{$\begin{array}{l}\text { It takes too much time to help } \\
\text { others who don't know how to } \\
\text { use the system }\end{array}$} & $\mathrm{N}$ & 9 & 29 & 37 & 87 & 43 & \multirow[b]{2}{*}{4} & 130 & \multirow[b]{2}{*}{2} \\
\hline & $\%$ & 4.4 & 14.1 & 18.0 & 42.4 & 21.0 & & 63.4 & \\
\hline \multirow{2}{*}{$\begin{array}{l}\text { I am aware that problems with } \\
\text { the EHR system have a direct } \\
\text { impact on patient care }\end{array}$} & $\mathrm{N}$ & 16 & 36 & 27 & 78 & 48 & \multirow[b]{2}{*}{4} & 126 & \multirow[b]{2}{*}{3} \\
\hline & $\%$ & 7.8 & 17.6 & 13.2 & 38.0 & 23.4 & & 61.4 & \\
\hline \multirow{2}{*}{$\begin{array}{l}\text { Using the EHR system takes } \\
\text { longer than the paper-based } \\
\text { system }\end{array}$} & $\mathrm{N}$ & 75 & 81 & 13 & 18 & 18 & \multirow[b]{2}{*}{2} & 36 & \multirow[b]{2}{*}{4} \\
\hline & $\%$ & 36.6 & 39.5 & 6.3 & 8.8 & 8.8 & & 17.6 & \\
\hline \multirow{2}{*}{$\begin{array}{l}\text { Using EHRS raises stress } \\
\text { levels among practitioners }\end{array}$} & $\mathrm{N}$ & 94 & 65 & 18 & 19 & 7 & \multirow{2}{*}{2} & 26 & \multirow{2}{*}{5} \\
\hline & $\%$ & 46.3 & 32.0 & 8.9 & 9.4 & 3.4 & & 12.8 & \\
\hline \multirow{2}{*}{$\begin{array}{l}\text { The system makes me feel like } \\
\text { I am no longer functioning as } \\
\text { part of a team }\end{array}$} & $\mathrm{N}$ & 73 & 85 & 21 & 15 & 10 & \multirow[b]{2}{*}{2} & 25 & \multirow[b]{2}{*}{6} \\
\hline & $\%$ & 35.8 & 41.7 & 10.3 & 7.4 & 4.9 & & 12.3 & \\
\hline \multirow{2}{*}{$\begin{array}{l}\text { The EHRS is considered to be } \\
\text { an extra load at work }\end{array}$} & $\mathrm{N}$ & 99 & 66 & 20 & 13 & 7 & \multirow{2}{*}{2} & 20 & \multirow{2}{*}{7} \\
\hline & $\%$ & 48.3 & 32.2 & 9.8 & 6.3 & 3.4 & & 9.7 & \\
\hline
\end{tabular}

The above four tables show a high variability in relation to EHRS end-user attitudes toward the use and implementation of the system. Whilst high levels of dissatisfaction were recorded, regarding organisational factors such as training, support and user involvement high levels of satisfaction were found in relation to the technology itself, such as ease of use, perceived usefulness, and efficiency.

\subsubsection{Barriers to EHRS implementation}

The responses to the sixteen questions on the barrier scale were mixed, with some factors being viewed as less of a barrier than others (see Table 6.3.13). The items given the lowest level of positive endorsement were: 1) "Lack of training" (83.8\%); 2) "Inadequate infrastructure and the absence of connectivity" (78.5\%); 3) "Lack of technical support" 
(75.5\%) and;4) "The absence of end-user involvement in EHR implementation and software design" (74.6\%).

The items that were given the highest level of positive endorsement and reflected lesser barriers were: 13) "The time spent using the EHR system" (34\%); 14) "Confidentiality and privacy concerns" (28.3\%); 15) "Concerns about loss of personal attention given to patients as entering patient information into the computer" (28.3\%) and; 16) "Concern about a decrease in productivity during the use of the EHR system" (25.4\%). As seen in the below table, all listed items in the barrier scale were considered to be major or minor barriers. 
Table 6.3.13: Participant responses to sixteen items representing barriers to EHRS implementation

\begin{tabular}{|c|c|c|c|c|c|c|}
\hline Items & & $\begin{array}{l}\text { Not a } \\
\text { barrier }\end{array}$ & $\begin{array}{l}\text { Minor } \\
\text { barrier }\end{array}$ & $\begin{array}{l}\text { Major } \\
\text { barrier }\end{array}$ & Median & Rank \\
\hline \multirow{2}{*}{ Lack of training } & $\mathrm{N}$ & 4 & 29 & 171 & \multirow{2}{*}{3} & \multirow{2}{*}{1} \\
\hline & $\%$ & 2.0 & 14.2 & 83.8 & & \\
\hline \multirow{2}{*}{$\begin{array}{l}\text { Inadequate infrastructure and } \\
\text { the absence of connectivity }\end{array}$} & $\mathrm{N}$ & 4 & 40 & 161 & \multirow{2}{*}{3} & \multirow{2}{*}{2} \\
\hline & $\%$ & 2.0 & 19.5 & 78.5 & & \\
\hline \multirow{2}{*}{ Lack of technical support } & $\mathrm{N}$ & 7 & 43 & 154 & \multirow{2}{*}{3} & \multirow{2}{*}{3} \\
\hline & $\%$ & 3.4 & 21.1 & 75.5 & & \\
\hline \multirow{2}{*}{$\begin{array}{l}\text { The absence of end-user } \\
\text { involvement in the EHR system } \\
\text { implementation and software } \\
\text { design }\end{array}$} & $\mathrm{N}$ & 9 & 43 & 153 & \multirow[b]{2}{*}{3} & \multirow[b]{2}{*}{4} \\
\hline & $\%$ & 4.4 & 21.0 & 74.6 & & \\
\hline \multirow{2}{*}{$\begin{array}{l}\text { Ignoring end-user feedback and } \\
\text { issue reports }\end{array}$} & $\mathrm{N}$ & 9 & 44 & 152 & \multirow{2}{*}{3} & \multirow{2}{*}{5} \\
\hline & $\%$ & 4.4 & 21.5 & 74.1 & & \\
\hline \multirow{2}{*}{$\begin{array}{l}\text { The lack of awareness of end- } \\
\text { user requirements }\end{array}$} & $\mathrm{N}$ & 14 & 73 & 118 & \multirow{2}{*}{3} & \multirow{2}{*}{6} \\
\hline & $\%$ & 6.8 & 35.6 & 57.6 & & \\
\hline \multirow{2}{*}{ Difficulties using the system } & $\mathrm{N}$ & 31 & 93 & 80 & \multirow{2}{*}{2} & \multirow{2}{*}{7} \\
\hline & $\%$ & 15.2 & 45.6 & 39.2 & & \\
\hline \multirow{2}{*}{$\begin{array}{l}\text { Inadequate resources and } \\
\text { materials within the centre }\end{array}$} & $\mathrm{N}$ & 12 & 113 & 79 & \multirow{2}{*}{2} & \multirow{2}{*}{8} \\
\hline & $\%$ & 5.9 & 55.4 & 38.7 & & \\
\hline \multirow{2}{*}{ Lack of computer literacy } & $\mathrm{N}$ & 25 & 103 & 77 & \multirow{2}{*}{2} & \multirow{2}{*}{9} \\
\hline & $\%$ & 12.2 & 50.2 & 37.6 & & \\
\hline \multirow{2}{*}{$\begin{array}{l}\text { Lack of perceived EHR system } \\
\text { usefulness }\end{array}$} & $\mathrm{N}$ & 37 & 92 & 76 & \multirow[t]{2}{*}{2} & 10 \\
\hline & $\%$ & 18.0 & 44.9 & 37.1 & & 10 \\
\hline Lack of awaronoss of EHRS & $\mathrm{N}$ & 32 & 99 & 74 & 2 & 11 \\
\hline & $\%$ & 15.6 & 48.3 & 36.1 & 2 & 11 \\
\hline Rocistanco to now tochnoloov & $\mathrm{N}$ & 29 & 105 & 71 & 2 & 12 \\
\hline Keststance to new lechnology & $\%$ & 14.1 & 51.2 & 34.6 & 2 & 12 \\
\hline The time spent using the EHR & $\mathrm{N}$ & 36 & 98 & 69 & 2 & 12 \\
\hline system & $\%$ & 17.7 & 48.3 & 34.0 & 2 & 13 \\
\hline Confidentiality and privacy & $\mathrm{N}$ & 51 & 96 & 58 & 2 & 14 \\
\hline concerns & $\%$ & 24.9 & 46.8 & 28.3 & & \\
\hline Concerns about loss of personal & $\mathrm{N}$ & 45 & 102 & 58 & & \\
\hline $\begin{array}{l}\text { attention given to patients as } \\
\text { entering patient information into } \\
\text { the computer }\end{array}$ & $\%$ & 22.0 & 49.8 & 28.3 & 2 & 15 \\
\hline Concern about a decrease in & $\mathrm{N}$ & 38 & 115 & 52 & & \\
\hline $\begin{array}{l}\text { productivity during the use of the } \\
\text { EHR system }\end{array}$ & $\%$ & 18.5 & 56.1 & 25.4 & 2 & 16 \\
\hline
\end{tabular}




\subsubsection{Participant responses to open-ended questions}

\section{Q1: What are the main things you like about the EHRS?}

The responses received to the open-ended question, "What are the main things you like about the EHRS?", were categorised into seven themes. In terms of system benefits, the aspects the EHRS end-users liked most about the system were timesaving, improved productivity, and easier access to data. Furthermore, EHRS end-users were very satisfied with the usability of the system, with participants saying that it was easy to use. A total of sixty-seven responses were obtained from the participants and categorised into seven themes (see Table 4.3.14).

The most frequent positive noted by EHRS end-users was related to usability, which constituted $31 \%(n=21)$. This was followed by time-savings, which constituted what $19 \%$ $(n=13)$ of EHRS end-users like about the system. In this theme, the EHRS end-users stated their appreciation of the role of the system in reducing time spent. Sixteen percent $(n=11)$ of EHRS end-users stated their most appreciated aspect of the system to be related to improved productivity. This was followed by data accessibility, which appears nine times (13\%). Patient privacy was the lowest ranked item that EHRS end-users reported to appreciate about the system, which appears only three times (4\%). In this theme, the EHRS end-users stated that only authorised people can access patient data. 
Table 6.3.14: What are the main things you like about the EHRS?

\begin{tabular}{|c|c|c|c|c|}
\hline Rank & Themes & Example of responses & $\begin{array}{l}\text { Frequency of } \\
\text { endorsement }\end{array}$ & Percentage \\
\hline 1 & Easy to use & $\begin{array}{c}\text { "Very easy" } \\
\text { "There is no need for } \\
\text { training to use it; it is very } \\
\text { clear." } \\
\text { "I like working on the } \\
\text { system; it is very easy." }\end{array}$ & 21 & $31 \%$ \\
\hline 2 & Time reduction & $\begin{array}{l}\text { "I spend less time when } \\
\text { using the system." } \\
\text { "Saves our time" } \\
\text { "I like the system because it } \\
\text { is faster than paper." }\end{array}$ & 13 & $19 \%$ \\
\hline 3 & $\begin{array}{l}\text { Improved } \\
\text { productivity }\end{array}$ & $\begin{array}{c}\text { "I feel my productivity is } \\
\text { better when using the } \\
\text { system." } \\
\text { "Our outcomes are } \\
\text { improved." }\end{array}$ & 11 & $16 \%$ \\
\hline 4 & $\begin{array}{c}\text { Data } \\
\text { accessibility }\end{array}$ & $\begin{array}{l}\text { "I can access the patient's } \\
\text { data from my computer." } \\
\text { "The data is always } \\
\text { available, even when the } \\
\text { patient's record is in } \\
\text { another clinic." }\end{array}$ & 9 & $13 \%$ \\
\hline 5 & $\begin{array}{c}\text { Improved } \\
\text { quality of care }\end{array}$ & $\begin{array}{l}\text { "It serves the patients } \\
\text { better." } \\
\text { "I can depend on the system } \\
\text { more compared with paper." } \\
\text { "Improves patient care" }\end{array}$ & 5 & $7 \%$ \\
\hline 6 & Accuracy & $\begin{array}{c}\text { "The data displayed on the } \\
\text { screen is much clearer than } \\
\text { on paper." } \\
\text { "It is not possible to make } \\
\text { errors when reading the } \\
\text { data." }\end{array}$ & 5 & $7 \%$ \\
\hline 7 & Patient privacy & $\begin{array}{l}\text { "Only authorised persons } \\
\text { can access patient records." } \\
\text { "We can monitor the } \\
\text { employees to see if there is } \\
\text { any misuse of data." }\end{array}$ & 3 & $4 \%$ \\
\hline & Total & & 67 & $100 \%$ \\
\hline
\end{tabular}




\section{Q2: Do you have any recommendations for the decision-makers to improve EHRS implementation?}

The recommendations by participants in response to the open-ended question, "Do you have any recommendations for the decision-makers to improve EHRS implementation?" were comprehensive and covered all the areas that affected the implementation process. A total of 127 responses were obtained from the participants and categorised into ten themes. The percentage of responses varied from one theme to another (see Table 6.3.15). The most frequent recommendations were related to training, which constituted $20 \%$ $(n=26)$ of the recommendations. In this theme, the EHRS end-users recommended providing adequate training programmes inside their workplace and during work hours. This was followed by recommendations for sufficient technical support, which constituted $18 \%(n=23)$ of the recommendations. In this theme, the PHCs staff suggested providing ongoing technical support and responses to their reporting of technical issues. $12 \%(n=15)$ of the recommendations were related to EHRS end-user involvement. On the technical side, $11 \%(\mathrm{n}=14)$ of the recommendations suggested improving the connectivity of the PHCs. EHRS end-users demanded connecting PHCs to the Internet and then connecting the PHCs to each other to share patient data. Similar to connectivity, recommendations to upgrade the hardware in the PHCs appear fourteen times (11\%). The lowest ranked recommendation was adding Clinical Decision Support System (CDSS) tools to the EHRS, which appeared only five times (4\%). In this theme, the EHRS endusers suggest adding CDSS to reduce medication errors. 
Table 6.3.15: Do you have any recommendations for the decision-makers to improve the implementation of the EHRS?

\begin{tabular}{|c|c|c|c|c|}
\hline Rank & Themes & Examples of responses & Frequency & Percentage \\
\hline 1 & Training & $\begin{array}{c}\text { "Increase training sessions" } \\
\text { "Training should be during work } \\
\text { hours." } \\
\text { “We need proper training.", } \\
\text { "Provide training on site." } \\
\text { "Provide qualified trainers." }\end{array}$ & 26 & $20 \%$ \\
\hline 2 & $\begin{array}{l}\text { Technical } \\
\text { support }\end{array}$ & $\begin{array}{c}\text { "Provide technical support } \\
\text { through direct lines to the } \\
\text { technical support department." } \\
\text { "Technical support is very } \\
\text { important." } \\
\text { "Provide ongoing technical } \\
\text { support." } \\
\text { "Solve technical issues." } \\
\text { "Respond to issue reports." }\end{array}$ & 23 & $18 \%$ \\
\hline 3 & $\begin{array}{l}\text { Involve the end- } \\
\text { user }\end{array}$ & $\begin{array}{c}\text { "They should listen to the users, } \\
\text { requirements and suggestions." } \\
\text { "We want to participate in the } \\
\text { system design." } \\
\text { "Involve those who will actually } \\
\text { work with the system." }\end{array}$ & 15 & $12 \%$ \\
\hline 4 & Connectivity & $\begin{array}{c}\text { "Link all PHCs together." } \\
\text { "Use E-referral between PHCs } \\
\text { and hospitals." } \\
\text { "Connect the system to the } \\
\text { Internet to exchange patient data } \\
\text { with other organisations." }\end{array}$ & 14 & $11 \%$ \\
\hline 5 & Hardware & $\begin{array}{l}\text { "Update the old computers." } \\
\text { "Change our computers." } \\
\text { "Provide computers for } \\
\text { everyone in the centre." }\end{array}$ & 14 & $11 \%$ \\
\hline 6 & Improvements & $\begin{array}{c}\text { "The current system needs some } \\
\text { enhancements." } \\
\text { "Improve the system." } \\
\text { "The system needs ongoing } \\
\text { improvement." } \\
\text { "Update the system on a regular } \\
\text { basis." }\end{array}$ & 11 & $9 \%$ \\
\hline 7 & System security & $\begin{array}{c}\text { "Concerns about the security of } \\
\text { the system." } \\
\text { "The system is not secure } \\
\text { enough." } \\
\text { "Security is very important." }\end{array}$ & 7 & $6 \%$ \\
\hline 8 & Inclusiveness & $\begin{array}{l}\text { "The system should be } \\
\text { comprehensive and serve all } \\
\text { departments at the centre." }\end{array}$ & 6 & $5 \%$ \\
\hline
\end{tabular}




\begin{tabular}{|c|c|c|c|c|}
\hline & & $\begin{array}{c}\text { "Add all medical orders to help } \\
\text { physicians." } \\
\text { "To benefit from the system, } \\
\text { specific tools must be added." }\end{array}$ & & \\
\hline 9 & $\begin{array}{l}\text { Technical } \\
\text { requirements }\end{array}$ & $\begin{array}{c}\text { "Automatically update the date } \\
\text { rather than manually every } \\
\text { day." } \\
\text { "Reduce the number of } \\
\text { screens." } \\
\text { "Make it easier to switch from } \\
\text { English to Arabic." } \\
\text { "Add an option to produce } \\
\text { medical reports." } \\
\text { "Update the vaccinations form } \\
\text { and all other forms in the } \\
\text { system." } \\
\text { "Add tools to follow-up on } \\
\text { patients with chronic diseases." }\end{array}$ & 6 & $5 \%$ \\
\hline \multirow[t]{2}{*}{10} & CDSS & $\begin{array}{c}\text { "I hope they add CDSS to help } \\
\text { us with medication." } \\
\text { "Better to include CDSS to } \\
\text { reduce medication errors." } \\
\text { "Our problem is duplication in } \\
\text { medication, so we need to have } \\
\text { CDSS." }\end{array}$ & 5 & $4 \%$ \\
\hline & Total & & 127 & $100 \%$ \\
\hline
\end{tabular}




\subsection{Inferential statistics}

\subsubsection{Demographic variable differences and their influence on scales}

As illustrated in Chapter Two, EHRS end-user demographics influence their level of satisfaction in different ways. The following represents inferential statistics that can be used to generalise outcomes from the sample, in this study, to the wider population. Nonparametric tests were performed to determine differences in the level of EHRS end-user satisfaction.

\section{Examining differences relating to gender}

A Mann-Whitney U test was used to measure whether or not the participants' gender had an effect on the way they responded to the items across the four scales. This test enables me to measure the difference between two independent groups (male and female in this case). Overall, it was evident that gender had no significant effect on the way the participants responded to the items across the scales regarding the usefulness of EHRS $(p=0.559)$, positive attitude $(p=0.737)$, satisfaction $(p=0.113)$, negative attitude $(p 0.338)$ and, finally, barriers $(\mathrm{p}=0.432)$ (see Table 6.4.1).

\section{Examining differences relating to the use of an EHRS in the workplace}

A Mann-Whitney U test was deployed to measure whether or not using an EHRS in the workplace made any difference to responses to the items across the four scales. The results showed no significant differences between the two groups across four scales ( $\mathrm{p}>0.05)$, as follows: usefulness $(\mathrm{p}=0.829)$, positive attitude $(\mathrm{p}=0.477)$, training and support $(\mathrm{p}=0.092)$, negative attitude $(\mathrm{p}=0.990)$ (see Table 6.4.1).

Table 6.4.1: Mann-Whitney U test for gender and using EHRS at the workplace

\begin{tabular}{|l|c|c|c|c|}
\hline & $\begin{array}{l}\text { Perceived } \\
\text { usefulness of the } \\
\text { EHRS }\end{array}$ & $\begin{array}{l}\text { Positive } \\
\text { attitude }\end{array}$ & $\begin{array}{c}\text { Training } \\
\text { and support }\end{array}$ & $\begin{array}{c}\text { Negative } \\
\text { attitude }\end{array}$ \\
\hline $\begin{array}{l}\text { p.value for gender } \\
\text { p.value for using } \\
\begin{array}{l}\text { EHRS at } \\
\text { workplace }\end{array}\end{array}$ & .559 & .737 & .113 & .338 \\
\hline
\end{tabular}




\section{Examining differences relating to province}

The findings suggest that the region/city where the participants lived had no significant effect on their responses to the items across the scales ( $p>0.05$ ), which were the usefulness of EHRS ( $\mathrm{p}=0.726)$, positive attitude $(\mathrm{p}=0.197)$, training and support $(\mathrm{p}=0.610)$, negative attitude ( $\mathrm{p}=0.221)$ and (see Table 6.4.2).

\section{Examining differences relating to occupation}

When examining differences relating to occupation a Kruskal-Wallis test detected only one significant difference between participants in regard to their occupation when looking at positive attitude $(\mathrm{p}=0.006)$. On the other hand, a Kruskal-Wallis test showed no significant difference between participants in regard to their occupation when looking at other scales: perceived usefulness $(\mathrm{p}=0.520)$, training and support $(\mathrm{p}=0.166)$ and negative attitude $(\mathrm{p}=0.964)$ (see Table 6.4.2).

\section{Examining differences relating to years of job experience}

Furthermore, I also tested whether or not job experience (in years) had an impact on the participants' responses to the items across the four scales and found that there was no significant effect ( $p>0.05)$ across all the variables, as follows: the usefulness of EHRS ( $p$ $=0.770)$, positive attitudes $(\mathrm{p}=0.204)$, training and support $(\mathrm{p}=0.765)$, and negative attitudes $(\mathrm{p}=0.833)$ (see Table 6.4.2).

\section{Examining differences relating to experience with using an EHRS}

I also examined whether experience using an EHRS had an impact on the participants' responses to the items across the four scales. The results indicated that experience using an EHRS had no significant effect ( $p>0.05$ ). No significant effect was found on the usefulness of an EHRS ( $\mathrm{p}=0.309)$, positive attitude $(\mathrm{p}=0.373)$, training and support $(\mathrm{p}=0.303)$, and negative attitude $(\mathrm{p}=0.570)$ (see Table 6.4.2).

\section{$\underline{\text { Examining differences relating to experience with using a personal computer }}$}

This section tested whether experience using personal computers had an impact on the participants' responses to the items across the four scales. The results indicated that there 
was no significant effect across all the scales ( $p>0.05)$, as follows: usefulness ( $p=0.598)$, positive attitude $(\mathrm{p}=0.590)$, training and support $(\mathrm{p}=0.804)$, and negative attitude ( $\mathrm{p}$ $=0.218)($ see Table 6.4.2) .

\section{Examining differences relating to age}

Overall, the results showed that age had no significant effect on responses to items across the scales ( $p>0.05)$, as follows: usefulness $(\mathrm{p}=0.312)$, positive attitude $(\mathrm{p}=0.470)$, training and support ( $\mathrm{p}=0.379)$, and negative attitude $(\mathrm{p}=0.542)$ (see Table 6.4.2).

Table 6.4.2: Kruskal-Wallis test for province, experience in position, experience in using an EHRS, experience in using personal computer, and age

\begin{tabular}{|l|c|c|c|c|}
\hline & Usefulness & $\begin{array}{c}\text { Positive } \\
\text { attitude }\end{array}$ & $\begin{array}{c}\text { Training } \\
\text { and support }\end{array}$ & $\begin{array}{c}\text { Negative } \\
\text { attitude }\end{array}$ \\
\hline $\begin{array}{l}\text { p.value for occupation } \\
\text { p.value for regions }\end{array}$ & .520 & .006 & .166 & .964 \\
\hline $\begin{array}{l}\text { p.value for experience } \\
\text { in position }\end{array}$ & .726 & .197 & .610 & .221 \\
\hline $\begin{array}{l}\text { p.value for experience } \\
\text { with using an EHRS }\end{array}$ & .309 & .104 & .765 & .833 \\
\hline $\begin{array}{l}\text { p.value for experience } \\
\text { in using a personal } \\
\text { computer }\end{array}$ & .598 & .590 & .303 & .570 \\
\hline $\begin{array}{l}\text { p.value for age } \\
\end{array}$ & .312 & .470 & .379 & .218 \\
\hline
\end{tabular}

\subsubsection{Correlations between main themes in the questionnaire}

A Spearman's correlation test was performed to determine the relationship between main scales. The correlation test showed a positive and significant correlation between the perceived usefulness of an EHRS and a positive attitude towards the use of the EHRS (see Table 6.4.3). Conversely, there was a negative correlation between usefulness and a negative attitude towards EHRS implementation. Furthermore, a negative correlation between a positive attitude toward the EHRS and lack of training and support was found (see Table 6.4.3). However, all significant correlations are considered to be weak. 
Table 6.4.3: Spearman's correlation coefficient for correlations between the four attitude scales

\begin{tabular}{|c|c|c|c|c|c|}
\hline & & $\begin{array}{l}\mathcal{E} \\
\stackrel{\infty}{\infty} \\
\stackrel{\Xi}{\Xi} \\
\stackrel{\infty}{\infty} \\
\infty\end{array}$ & 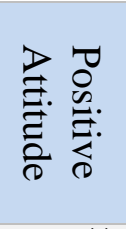 & 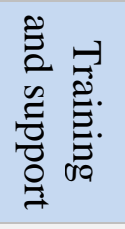 & 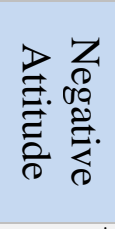 \\
\hline \multirow{3}{*}{ Usefulness } & Correlation Coefficient & 1.000 & $.239^{* * *}$ & -.055 & $-.176^{*}$ \\
\hline & Sig. (2-tailed) & 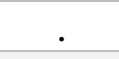 & .001 & .429 & .012 \\
\hline & $\mathrm{N}$ & 205 & 205 & 205 & 205 \\
\hline \multirow{3}{*}{ Positive attitude } & Correlation Coefficient & $.239^{* *}$ & 1.000 & $-.148^{*}$ & -.031 \\
\hline & Sig. (2-tailed) & .001 & & .034 & .657 \\
\hline & $\mathrm{N}$ & 205 & 205 & 205 & 205 \\
\hline \multirow{3}{*}{ Training and support } & Correlation Coefficient & -.055 & $-.148^{*}$ & 1.000 & -.051 \\
\hline & Sig. (2-tailed) & .429 & .034 & . & .467 \\
\hline & $\mathrm{N}$ & 205 & 205 & 205 & 205 \\
\hline \multirow{3}{*}{ Negative Attitude } & Correlation Coefficient & $-.176^{*}$ & -.031 & -.051 & 1.000 \\
\hline & Sig. (2-tailed) & .012 & .657 & .467 & \\
\hline & $\mathrm{N}$ & 205 & 205 & 205 & 205 \\
\hline
\end{tabular}




\subsection{Summary}

The target population for this study were EHRS end-users in Saudi PHCs. The questionnaire was distributed to 483 people, and 205 of the questionnaires were returned, giving a $42.4 \%$ response rate. Similar to Studies One and Two, the majority of the participants were male (77.6\%), and a large proportion of participants were nurses $(32 \%)$. Most participants were between twenty-five and forty-four $(55.1 \%)$ years of age. This study was designed to evaluate the implemented EHRS in the Saudi PHCs and to determine the main barriers to EHRS implementation from an end-user perspective.

Regarding end-user attitudes toward the EHRS, there was variability in attitudes recorded across all three scales. Firstly, when looking at the positive attitude scales, it was clear that there was an overall positive attitude towards the EHRS. As for the negative attitude scale, there was clearly more disagreement with the negative statements, which further re-affirms the positive attitude of the participants. Negative attitude statements reflected negative opinions of the EHRS and, hence, disagreement with them reflects a positive attitude and that the participants generally welcome the EHRS. Attitude toward training and support generated negative responses, with the participants indicating dissatisfaction with the current EHRS across most items.

The responses regarding the main barriers to EHRS implementation in PHCs were mixed, with some factors being viewed as less of a barrier than others. For instance, training and support were recorded as a major barrier, while confidentiality and privacy were found to be less of a barrier to EHRS implementation. When looking at factors that may influence EHRS end-user satisfaction, with the exception of participants occupation, the inferential statistics showed that demographic characteristics had no significant impact on EHRS end-user attitudes and satisfaction toward the implementation and use of the system. However, participants in their different occupations expressed different positive attitudes toward the implementation of the EHRS. Correlation tests showed only one positive significant correlation between perceived usefulness and positive attitudes toward the EHRS implementation.

In response the open-ended questions, the participants raised several issues in regard to the infrastructure, such as concerns about connectivity and hardware. They expressed 
their appreciation of the system's benefits, such as improving productivity, patient privacy, data accuracy and data accessibility and reducing medication errors and time spent. In response to the open-ended questions that require further suggestions, the participants suggested that some improvements should be made to the system, such as adding more tools and orders to make the system more comprehensive and suited to the needs of all end-users from different specialities.

Although the implemented EHRS was quantitatively evaluated and presented in this chapter, the evaluation was based on an end-user perspective. Hence, the following chapter will provide in-depth information about the implemented EHRS from a project team perspective. The following chapter will also present findings from semi-structured interviews which include further details and in-depth descriptions of PHC readiness for EHRS implementation as well as the impact of CM and FR on EHRS implementation. 
7 Chapter Seven: Study Four: Semistructured Interviews Exploring the EHRS implementation in PHCs in SA from a Project Team Perspective 


\subsection{Introduction}

This study was designed to explore the implementation of an EHRS in Saudi PHCs from a project team perspective. The readiness assessment will take into consideration a description of the implementation plan and other factors influencing EHRS implementation in the pre-implementation phase. Moreover, this study aims to determine the main barriers and facilitators to EHRS implication from a project team perspective, and evaluate the impact of CM and FR. A thematic analysis was used to analyse the obtained data (see Chapter Three). The selection of the quotations was based on a line by line reading of the transcripts, and all quotations included in this chapter have been selected for their appropriateness to the study aims (see Chapter Three). The included quotations are directly related to the above-mentioned aims.

The participants were occupied in five different positions (see Table 7.1.1): General Manager ( $n=3)$, Head of Department ( $n=3)$, Deputy Head of Department ( $n=1)$, Software Developer ( $\mathrm{n}=1)$, and Analyst $(\mathrm{n}=3)$.

Table 7.1.1: Participant abbreviation description

\begin{tabular}{|l|l|}
\hline Position & Code used \\
\hline General Manager & GM \\
\hline Head of Department & HD \\
\hline Deputy Head of Department & DHD \\
\hline Software Developer & SD \\
\hline Analyst & Analyst \\
\hline
\end{tabular}

Figure 7.1.1 shows the most frequent words and terms reported during the interviews. "Systems" "health" and "centres" were the most frequently mentioned. The words included in Figure 7.1.1 are the most frequent words, other words in the transcripts are not available in the figure. 


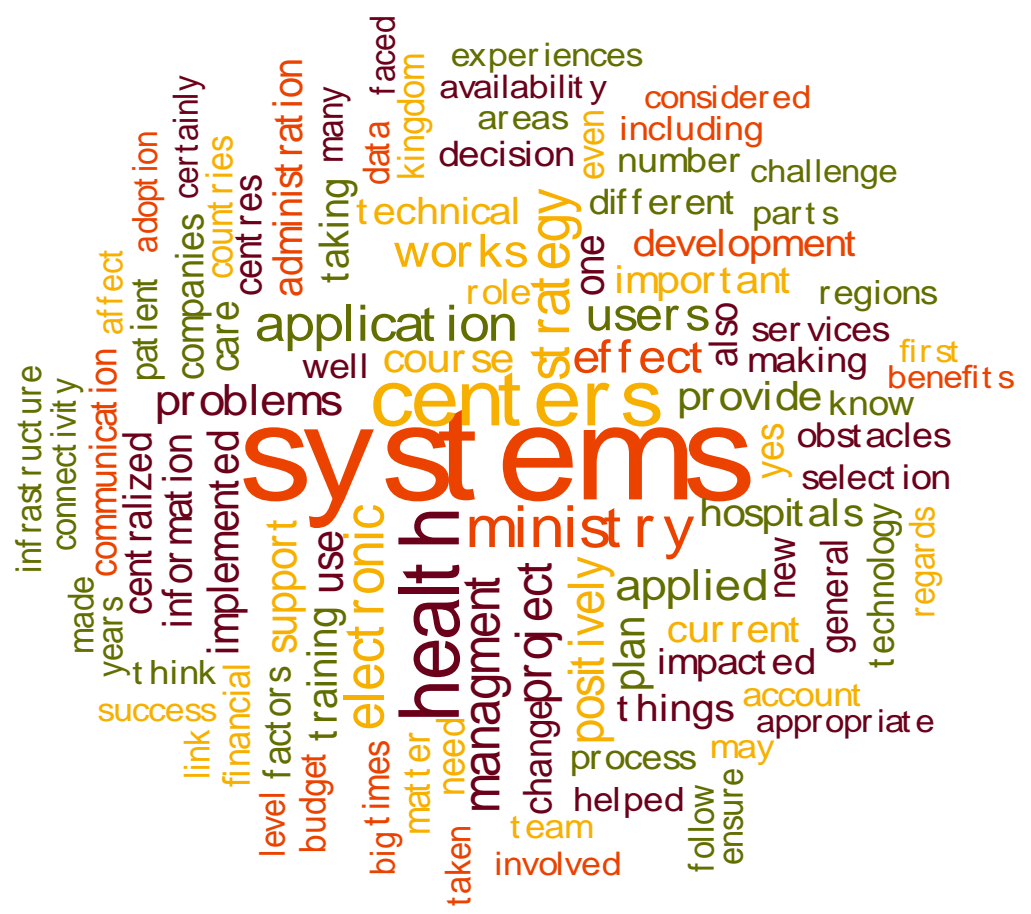

Figure 7.1.1: The most frequent words in the interviews

As seen in Table 7.1.2, thirty-eight key codes emerged from the analysis of the transcripts. Although the number of codes that emerged from the transcripts was greater than included in the table below, Table 7.1.2 includes the key codes only. All codes and sub-codes will be presented in the following sections. Six themes were phrased according to all codes that emerged from the transcripts:

- Procedures adopted in the pre-implementation phase.

- Plan for implementation of an EHRS in PHCs.

- The role of the Centralised Management (CM) in EHRS implementation.

- The role of Financial Recourses (FR) in EHRS implementation.

- Facilitators and barriers to EHRS implementation.

- The EHRS implemented in PHCs in SA. 
Table 7.1.2: List of key codes via NVivo 10

\section{No. Key Codes}

1. Restructuring and workflow redesign

2. Readiness assessments

3. Consultations

4. Project team selection

5. Consultation

6. Project team communication

7. Awareness campaigns

8. Management and leadership

9. Training

10. Technical support

11. Security, privacy and confidentiality

12. End-user involvement

13. User acceptance

14. User resistance

15. End-user requirements

16. Interoperability

17. Software selection

18. Usability

19. Efficiency

20. Infrastructure

21. Software selection

22. Financial support

23. Characteristics of the PHCs

24. Perceived usefulness

25. Changing people

26. Lack of experts

27. Number of PHCs

28. EHRS implementation time

29. Vendors

30. Lack of connectivity

31. Geographical challenges

32. Piloting the system

33. Co-operation with Telecommunications Companies (TCs)

34. Divide the country into regions

35. Studies and research

36. Technology developments

37. Evaluation

38. User awareness 


\subsection{Theme One: Procedures adopted in the pre-implementation phase.}

This section will describe EHRS pre-implementation phase procedures for PHCs in SA. It will be presented as a process based on the codes generated from the transcripts. The pre-implementation procedures began with selecting the project team. Once project team selection has been described the participants explained the communication mechanisms between project team. In addition, the participants explained how end-users and other stakeholders were involved. Thereafter, they underline how to maintain EHRS end-user acceptance. The interviewees also illustrated the process of readiness assessment, PHCs restructuring and workflow redesign.

\subsubsection{Project team selection}

The individuals on the project team were chosen carefully by the IT department at the Saudi MoH in accordance with their ability to carry out this task.

"In the implementation, well-qualified people were selected." (HD 3 )

"All the team members have been selected by the IT department and they are all highly qualified.” (HD 2)

The project team consists of three different levels: senior managers at the Saudi $\mathrm{MoH}$ level, and health affairs management at the regional and PHC level.

"The selection of project team members was from three levels: senior management at the Ministry, and then from the regions' management level, and finally, from the PHC level." (Analysts 2)

According to the software developer and one of the analysts, the project team consisted of doctors, nurses, technicians, laboratory IT technicians, engineers and administrators. In addition, the project team have experience in their field and others have experience in similar projects.

"This is natural, they were appointed as highly qualified people from different departments and expertise; so, we selected doctors and engineers, including a doctor who worked for ten years as a PHC manager and served us in the analysis of the current status of PHCs." (SD 1) 
"The team was composed of several different levels and backgrounds of doctors, nurses, administrators, lab technicians and centre managers." (Analyst 1)

Similarly, HD3 stated that the project team consist of people "from various departments and all levels."

Once the project team had been selected, the project team members started communication between themselves. The following section will describe the project team communication mechanisms.

\subsubsection{Project team communication}

The Saudi MoH has utilised methods to ensure proper communication between the project team members. Communication between the project team members is conducted through the formation of committees and the holding of workshops and meetings, and occurs either face-to-face at the headquarters of the $\mathrm{MoH}$, or via the Internet and other media.

\section{Communications are made via committee}

The formation of committees is one of the most common methods used by the Saudi MoH to conduct consultations and negotiations, as well as to make decisions related to EHRS implementation in PHCs in SA. Committees often consist of representatives from the relevant departments and the PHCs. In addition to direct encounters in person at the Ministry, members of the committees communicate either through e-mail or mobile phones.

"Committees have been formed to communicate with other project team members." (HD 3)

"... and set up specialised committees for this project with the involvement of representatives from different departments at the headquarters of the $\mathrm{MoH}$ " (GM 3)

Communication among all project team members is not limited to committees, workshops are also used to communicate between project team members. 


\section{Communications were made via workshops}

Project team communication workshops are held both inside the headquarters of the $\mathrm{MoH}$ to promote proper communication between project team members. The workshops are held on an ongoing basis.

“We hold continuous workshops.” (GM 2)

"One of the most important plans made by the Ministry to ensure good communication between the project team is holding repeated workshops and periodic meetings; often weekly." (Analyst 1)

Some workshops are even held outside work hours, which is reportedly the most effective method of communication for project team members.

"We hold the communication process and meetings and workshops outside of work hours." (GM 3)

\section{Communications made via meetings}

In addition to the formation of committees and the holding of workshops, the project team holds regular meetings to ensure communication between all members of the project team.

“Communication between teamwork through holding regular meetings.” (HD 2)

“We made regular meetings.” (SD 1)

\section{Communications made via media}

The typical method of communication between the project team is email, video conferencing and other media.

"We communicate through email as well as video conferences to facilitate communication between us." (DHD1)

“Communicate via e-mail as well as through mobile.” (HD3)

The interviewees also highlighted the importance of involving EHRS end-users and other stakeholders in the above-mentioned communication methods. The following section will describe the EHRS end-users and other stakeholders' mechanisms. 


\subsubsection{EHRS end-user involvement}

End-user involvement aims to enhance EHRS end-user satisfaction. Hence, EHRS endusers have been involved through committee appointed representatives. These representatives can be champions or super-users who act as a communication channel between the project team and the end-users (see Chapter Two).

"Representatives from the PHCs have been appointed for the representation of other users." (HD3)

"The aim of involvement is to reduce the rate of resistance and unwillingness to use the system." (GM3)

"The most important thing which has been taken into account to ensure the success of the EHRS implementation is the participation of all the beneficiaries of these systems during the strategy and noting their opinions and requirements." (HD 1)

"If users do not participate or share their opinions and needs, the project may fail, so they must be involved to overcome any barriers that may negatively influence their satisfaction." (HD 3)

According to HD 3, “...committees have been formed with the participation of representatives of end-users and the relevant department in the Ministry."

The involvement of EHRS end-users took place in the early stages of the EHRS implementation project. Thus, planning was formulated with consideration of the involvement of the stakeholders. However, end-user involvement was not limited to the early stages, but lasted throughout all phases of the project, even into the postimplementation phase.

"All beneficiaries of the system or stakeholders have been involved since planning and strategy development." (HD1)

"We have involved all stakeholders in the strategy; they were always participants in our meetings, and we are still carrying out these meetings and consultations between us. " (GM3)

On the other hand, the level of participation in the project varied from one phase to another. There was reasonable involvement in the process of decision-making in general. The MoH did not make any decisions concerning the implementation of EHRS without consulting all those involved; each according to his/her specialisation, particularly in 
clinical decisions. Inputs were taken from representatives of the PHCs from different backgrounds.

“All stakeholders were involved in decision-making." (Analyst 3)

"There is no decision made with respect to clinical matters without consultation and participation of a doctor or other technician who specialises in the same field." (HD 1)

In this context, the EHRS end-users or their representatives were particularly involved in the decision-making regarding software selection.

"Stakeholders were involved during the software selection." (HD 1)

"Whenever we want to select a new system, we should engage stakeholders or their representative." (GM 1)

As illustrated in Chapter Two, there is a correlation between EHRS end-user involvement and their acceptance of EHRS implementation. Therefore, the interviewees underline the importance of maintaining end-user acceptance in the early stages. The following section will describe the procedures followed to maintain end-user acceptance of EHRS implementation.

\subsubsection{EHRS end-user acceptance}

Human related factors were a focus of the project team during EHRS implementation.

“...in particular human factors; they are the most important factors that may contribute to the success or failure of EHRS implementation." (HD3)

For instance, EHRS end-user acceptance and satisfaction were found to be crucial to the success of the project.

"If the user doesn't want to use the system, then it will affect the success of the implementation." (Analyst 1)

Therefore, the Saudi MoH now attempts to prepare EHRS end-users for the changes. 
"Preparing them and trying to remove the fears of using the new technology." (HD 1)

The responses, obtained from some of the participants, indicated that the majority of users are very enthusiastic about the use of an EHRS and are motivated to make the transition to electronic transactions in their workplace.

"They are ready for the maximum extent because they need this system; electronic transactions are required to facilitate the performance of work. " (HD 3)

"Users are ready, and they are very enthusiastic about the system." (Analyst 1)

One of the encouragements, that contributed to the EHRS end-user's enthusiasm and acceptance, was the positive impact of technology on their daily lives.

"Most users were briefed on the technical side of things and they know the value of this technology and its impact on their daily lives." (GM 3)

\subsubsection{Awareness campaigns}

To prepare the end-users for the new EHRS and reduce their resistance to it, awareness campaigns will be carried out and occur side by side with training in the implementation process. The awareness campaigns will be presented continuously throughout all implementation phases.

"There will be awareness campaigns and guidance during the implementation period." (HD3)

In this context, the delivery methods used for awareness campaigns will be through distributing brochures and leaflets explaining the EHRS project, as well as the intended benefits of the system. In addition, there will be visits to the PHCs to introduce the implementation of the EHRS project.

"At the beginning, there will be the provision of promotional material such as leaflets.” (HD 1)

"...brochures will be distributed prior to the implementation and visits to these PHCs will be held to introduce the EHRS."

(HD2) 
However, a general manager argued that the Saudi MoH "In marketing; is not strong enough." (GM1)

The awareness campaigns highlight the potential benefits of the EHRS and the advantages of its implementation. The EHRS end-user should know why the EHRS system will be implemented in their workplace and what the potential benefits of the system are to encourage them to use the system and to avoid any resistance that may occur in the future. "...will give an idea about the project and the potential benefits." (DHD1)

"We will organise awareness campaigns to inform the users of the system and its advantages and its role to facilitate their work."

(Analyst 1)

\subsubsection{Readiness assessments}

Healthcare organisations readiness to implement a new EHRS has been found to be influential to the success of the project (see Chapter Two). Therefore, the Saudi MoH take several readiness measurements to ensure successful EHRS implementation.

"Some of the requirements have been considered, such as the readiness of PHCs to successfully implement new technology.” (GM 1)

Numerous studies were conducted in order to identify all the obstacles and facilitators directly associated with the success or failure of EHRS implementation projects. In addition, the Saudi MoH conducted research to determine the strengths and weaknesses that may influence EHRS implementation in PHCs in SA

"Firstly, we made so many studies prior to the implementation to know the strengths and weaknesses of the challenge." (DHD 1)

"We conducted a number of studies and research before the beginning of any EHRS implementation to learn the strengths and weaknesses, as well as to determine the challenges, the causes of failure and identify risks." (HD 3)

"The Ministry conducted research to avoid any problems and ensure the successful implementation of the EHRS." (GM 3) 
At a technological level, the $\mathrm{MoH}$ is currently assessing the readiness of PHCs, in regard to the connectivity infrastructure, to accommodate the EHRS implementation project and identifying any obstacles and challenges.

"We discussed the readiness of hospitals and PHCs in terms of infrastructure compatibility with the change, especially PHCs. ” (HD 3)

"We are currently making a situation analysis of the PHCs, including an assessment of the PHCs readiness in terms of connectivity." (GM 3 )

While some of the participants believe all PHCs are currently ready for EHRS implementation.

"PHCs are currently ready to implement the EHRS." (DHD1)

“I think they are almost ready to use a new EHRS." (HD 3)

Others think that PHCs are not yet ready to implement the EHRS.

"I think some of the PHCs are still not ready for this project, compared with hospitals." (SD 1)

"Some of the PHCs need to be prepared for the new implementation - they are not ready yet." (GM1)

\subsubsection{Restructuring and workflow redesign}

In the pre-implementation phase, the Saudi $\mathrm{MoH}$ are planning to re-structure the workflow of the PHCs to comply with new changes post EHRS implementation. This is confirmation that the $\mathrm{MoH}$ is restructuring the workflow of some of its centres that use EHRS. Although there will be three different vendors implementing EHRS in Saudi PHCs, they will take the role of redesigning a unified workflow. According to one of the participants, the workflow redesign will not be affected by the fact that the Ministry will select three different vendors.

"Another thing that we could take into consideration is to redesign the PHC workflow; even if we do have three vendors, we will ask them to design one workflow for all PHCS." (HD1)

"If you go to the PHCs that have the EHRS and other centres, you will see that they have been redesigned to comply with new IT projects." (GM1) 
This section included all codes generated from the transcripts that described the preimplementation phase procedures. According to the participants' responses, the first step in the pre-implementation phase was to select the project team. Team members were selected based on several criteria: background, experience, position, and profession. Once the project team had been built, communication was established through meetings, workshops, committees and other media including email. It has been illustrated that EHRS end-users or their presentative have been involved in these meetings and other communication methods. Initially, the involvement of EHRS end-users will take place in the pre-implementation phase and will continue through to the implementation and postimplementation phases. They will be involved in the decision-making processes, in particular software selection. Furthermore, user acceptance has been found to be essential to the successful implementation of an EHRS. Therefore, the findings show that the plan includes procedures to improve user acceptance and reduce their resistance to EHRS implementation.

The project team planned to launch awareness campaigns, prior to implementation, to prepare the EHRS end-users and enhance their acceptance. Moreover, the participants revealed that PHC readiness for an EHRS has been considered in the early stages of implementation (pre-implementation phase), particularly readiness at a technical level, such as preparedness of the infrastructure. Participants also stated that, one of the preimplementation procedures, was to restructure the PHCs to comply with the new EHRS. 


\subsection{Theme Two: Plan for EHRS implementation in PHCs.}

This theme describes in detail the process to formulate a plan to implement a new EHRS in PHCs. The participants highlighted the main elements of the plan as well as the process. Initially, everything is still on paper and no practical steps have been taken. The Saudi $\mathrm{MoH}$ has developed an elaborate scheme for the implementation based on the principle of priority, whereby projects are implemented in certain PHCs according to preliminary needs.

"All that has been done before is a theoretical thing on paper; there has been no action taken or a trial for certain systems." (GM 2)

The essential stage in the planning phase was to conduct several consultations at various levels.

\subsubsection{Consultations during the formulation of the EHRS implementation plan}

In this section, the participants describe the need for consultation with this type of project and how such consultations are conducted. The Saudi MoH has carried out several different consultations to enhance the implementation plan, conducted on three different levels: country, organisational, and individual See Figure 7.3.1.

Consultations at country level:

Canada, Australia, Turkey, Norway, Jordan, Denmark, United Kingdom, United States, South Korea and Singapore

Consultations at organisational level:

Health Info Way and IBM

Consultations at individual level:

Reviwers, auditors and consultants from SA and some developed countries such as UK and Australia

Figure 7.3.1: Type of consultations 


\section{Consultations made at the country level}

Although the Saudi MoH has conducted consultations in different countries, the nature of Saudi PHCs business process and workflow is different to that of other countries, as this participant's comment highlights:

"We benchmark with the Canadian experience, and we also had a benchmark with some countries like Australia, Turkey and Denmark, which have big initiatives." (GM 1)

"In addition to America, we have a number of trips and visits to a group of countries in Europe (Norway, Spain, Germany, Italy), North America (Canada) and South Korea. We have access to all the systems they already have, and we have benefitted from their experiences in this area." (HD 1)

"Moreover, we benefited from the experiences of other countries like Norway and Australia." (DHD 1)

"The Ministry benefited from the South Korean experience as well as the Jordan experience." (Analyst 1)

"Most of the benefits that we have are from the UK experience; the UK experience is a very rich experience with a lot of difficulties and failures, so it was very rich, and we learned from it." (GM 1)

The consultation-based reviews varied from country to country, depending on several criteria. One of the criteria considered was the resemblance of the Saudi healthcare system to that of other countries. The healthcare system of some countries, such as Canada, is considered closest to the Saudi healthcare system. Thus, there is great co-operation between health institutions in SA and Canada in this context. Subsequently, there is Australia, where the healthcare system is the second most similar to that of Saudi Arabia. Singapore occupies third place in terms of similarities with the SA healthcare system. Therefore, these countries are the most involved in the consultation process based on the statements of the participants; for example:

“The countries most involved are Australia and Singapore." (Analyst 3)

"...especially since Canada is the nearest country to us in terms of its healthcare system and sharing some of the challenges such as cities that are a great distance from each other, so there was co-operation between us directly and continuously." (HD 1) 
As stated by a head of department, "It is not logical to use other countries' strategies and apply them to SA, or even take some aspects" (HD 1). Therefore, the purpose of the consultations was to take advantage of the positive experiences of different countries, regardless of the differences in healthcare provision and organisation. The countries that were successful in implementing their strategies are the UK, Canada and America; as indicated in the following comments:

"Definite strategy in the beginning was to bring distinctive resources of the strategies that were successful, such as those of Britain, Canada and America." (HD 2)

\section{Consultations made at the organisational level}

The Saudi MoH did not implement and apply strategies from other countries, but they benefitted from the experiences of some companies (national and international), which had extensive experience in this field and a willingness to cooperate with the $\mathrm{MoH}$ to create and develop a strategy that was suited to companies in Saudi Arabia. Therefore, consultations were not confined to the country level, but were also held at the organisational level. Those organisations played a huge role in the formulation and development of the Saudi e-health strategy. Consequently, the co-operation established a roadmap to facilitate the implementation of EHRS in PHCs in Saudi Arabia; as these comments illustrate:

"There is no use of outside strategies directly, but we used well-known and large global companies. They have a great experience in this area and they help to set up the private strategy of the Saudi Arabia Ministry of Health, but they did not use other strategies because the e-health strategy of the Ministry of Health was made here in the Ministry, and this strategy won international awards." (HD 1)

At organisational level, the "Health Info Way" in Canada was chosen for the establishment and development of the Saudi e-health strategy, in general, and the implementation of EHRS in particular. At the beginning of the co-operation, the Saudi MoH created a Request for Proposal (RFP) in order to determine the criteria, requirements and regulations, as evidenced by the following comments from two participants: 
"We built our Request for Proposal (RFP) and sent it to 'Health Info Way' in Canada to give it to their consultants; we worked with them." (GM 1)

“We benefitted from 'Health Info Way' in Canada." (DHD 1)

Among the organisations, that have been cooperating with them and have had a direct involvement in the implementation of EHRS, is IBM. The role of IBM is concentrated on the review and implementation of the Saudi e-health strategy and all the projects adopted in the strategy.

"IBM was responsible for the development of the e-health strategy." (Analyst 2)

This was further supported by comments from a deputy head of department:

"The Health Ministry annually reviewed the e-health strategy to assure the smooth running of the projects. The last review was run by the Ministry in 2014 during the convention of HIMSS, which was held in Jeddah this year in cooperation with a consulting committee and IBM." (DHD 1)

“We have co-operation with the IBM company." (DHD 1)

\section{Consultations made at the individual level}

The Saudi MoH also conducted consultations at the individual level. However, this time the MoH did not rely on international-based experts only, they hired experts from both inside and outside the Kingdom. The task of the internal consultants was to review the decisions made and the strategies; as illustrated in the comments below:

"Internally we have six reviewers from the Kingdom here." (GM 1)

“...was attended by a number of consultants within the Saudi Ministry.” (GM 3)

Numerous important issues were discussed with consultants and experts from inside and outside the Kingdom.

"A lot of reasons for failure are discussed and challenges that may hinder the implementation of the EHRS are also discussed with them (experts)." (HD 3).

In addition to the internal reviewers, external reviewers from developed countries were hired to review the EHRS implementation plan, those reviewers had extensive experience in their countries and had participated in successful projects. 
"The Ministry used international auditors and senior experts who have experience in this field." (HD 1)

"It was better to use the same experts who participated in successful strategies in their countries and ask them to participate in reviewing the Saudi E-Health Strategy.”(HD 1)

The consultants and experts who were involved came from different countries such as Canada, USA, UK and Australia, and had extensive experience in this sort of project. The consultations were conducted either by the consultants themselves attending the headquarters of the Ministry, or by communicating with them via the Internet; as illustrated by the comments below:

"We have learned a lot from consultants who have been hired, whether through contracts or being present in the Kingdom, or by communicating with them in their own countries, whether from Canada, Australia or other countries that have experience in these projects. We have been directed by them to do the proper mechanism and how to avoid mistakes." (HD 3)

"We had contact with experts in the field of EHRS implementation from different countries, including USA, Australia and UK.” (DHD 1)

The selection criteria of the experts and consultants were not limited to their expertise and nationalities. The consultants' and experts' backgrounds, interests and specialties were also taken into consideration.

"During the implementation of the system there has been hiring from different backgrounds; different specialties were taken into account. The two parts I had were either from a clinical background or from a technical background." (DHD 1)

"In the planning, qualified people from different backgrounds have been chosen, and from outside the Kingdom." (HD 3)

In addition to the consultations made during the formulation of the plan, the project team also considered the EHRS end-user requirements. The following section illustrates the consideration of EHRS end-user requirements during the planning phase. 


\subsubsection{End-user requirements}

The importance of EHRS end-user participation, during the planning and EHRS selection phase, was illustrated by the participants. The project team at the Saudi MoH pays attention to end-user requirements during planning and software selection.

"User needs have been considered." (HD 1)

"The end-users' needs and requirements have been taken into account." (GM 2)

"End-users have been taking into account their requirements and needs during the planning." (HD 2)

Particularly during the software selection.

"Essentially, cannot build a system without understanding user requirements." (Analyst1)

"User needs are the foundation of our system selection criteria." (GM3)

\subsubsection{Software selection}

In addition to the consideration of end-user requirements, this section illustrates other software selection process and criteria to be considered during software selection. Software selection is one of the major challenges the $\mathrm{MoH}$ faces and can lead to delays in many projects, especially the implementation of EHRS in PHCs. Moreover, the participants reported some of the reasons for this obstacle, such as no available software meeting their requirements. One of the participants emphasised that the $\mathrm{MoH}$ had not yet found a system that complies with the functions of PHCs in Saudi Arabia. Therefore, the $\mathrm{MoH}$ is still keenly searching for a perfectly integrated system that meets the requirements and ambitions of the Ministry and is commensurate with the functions of the PHCs. According to a head of department, the Saudi MoH also considers other criteria, such as system efficiency and ease of use of the system, to be important. These two criteria are discussed in detail below.

“The system's ease of use and efficiency are considered." (HD 3) 


\section{$\underline{\text { System efficiency }}$}

The Saudi MoH considers system efficiency to be one of the requirements of an EHRS implementation project. The participants justified the concerns of the Saudi MoH in this regard, since software selection is one of the main reasons for the success or failure of EHRS implementation projects. The deputy head of department stated that if the efficiency of the system is poor, it will not be able to fulfil its purpose and will not meet the user's expectations, which may lead to user resistance.

"Efficiency of the system has been taken into account because if the system doesn't help users do their jobs, or if the system doesn't improve health services, user-acceptance will fail, and we may then go back to the paper-based system." (DHD 1)

"This is essential and one of the most important criteria that we have set for the selection of the system -the system's usability and efficiency." (GM 3)

\section{$\underline{\text { System usability }}$}

The Ministry included ease of use of the system as one of the essential criteria during software selection. To verify the ease of use of any EHRS, the project team is planning to request a trial version of the software from the vendors to test the system. This testing is performed with the coordination of actual users of the EHRS to measure their satisfaction with the system's usability.

"Ease of use, or so-called 'user friendliness', is a requirement for each user, and ease of use of the system is part of the criteria that has been set for the selection of any system." (Analyst 1)

"System usability is one of the most important criteria which we take into account during the testing of any system." (GM 3)

\section{$\underline{\text { EHRSs Interoperability }}$}

Interoperability of EHRS systems has also been considered as one of the main criteria during software selection. Participants mentioned two methods of enhancing EHRS interoperability: through the development of such a standard, and the development of terminology scheme. The Saudi $\mathrm{MoH}$ is planning to implement interoperable EHRS in PHCs. The purpose of this is to facilitate inter-PHC communication and exchange patient 
information without any technical problems. This will continue to facilitate the connectivity of PHCs with hospitals and all organisations affiliated to the Saudi $\mathrm{MoH}$, as well as to reduce the distance gap between the regions of the Kingdom of Saudi Arabia.

"A unified system can be implemented to avoid the problems of compatibility between systems, whether with the same health centres or with hospitals." (Analyst 2)

To enhance systems interoperability, the Saudi MoH is developing its own standard.

“We also started to build our own interoperability standard." (GM1)

In the same context, the Saudi MoH has worked on the development of two terminology schemes known as SnomedCT and ICD 10.

"The MoH developed two standards, known as MedCT and ICD 10, for data. They have worked on this for about two years." (Analyst 3)

\section{$\underline{\text { Local or international system }}$}

Whether to select a local or an international system is a subject of broad debate among project teams. However, most prefer international systems to local systems, hence the project team primarily endorse the implementation of an international EHRS. This is illustrated clearly in the responses of most of the project team.

“The Ministry has decided to buy an international system." (Analyst 1)

"The Ministry is looking globally to find a system that meets its requirements. We have to hold many meetings with large international companies that present their systems to the Ministry." (HD 1)

However, the head of department supports a decision to select a local system.

"One of the things proposed for discussion at the moment is to find a local system for the PHCs." (HD3)

\section{$\underline{\text { Request for Proposal (RFP) }}$}

The MoH has formulated an RFP that includes all criteria, requirements and conditions for the implementation of an EHRS. The IT department at the Saudi MoH co-ordinates 
with the relevant departments to draft the RFP and then provide the companies with a copy. In order to create an integral and clear RFP, the MoH has conducted a full analysis at the PHCs to determine their functions and requirements. Thereafter, the potential vendors who will implement the EHRS in the PHCs will obtain a copy of the RFP, in order to assess whether they are commensurate with requirements.

"The IT department team will provide the RFP to companies that have already submitted offers to consider whether their systems meet our requirements." (Analyst 2)

"A full situation analysis of PHCs has been added to the RFP, which contains all functions and services provided by the PHCs." (Analyst 2)

In the same context, a general manager emphasised the importance of issuing a clear and integral RFP document to avoid any problems during the implementation of EHRS.

"It is very risky if the RFP is not clear for the vendor; you may face implementation issues in the future." (GM1)

Nevertheless, no system had been selected at the time of the interviews. The Saudi MoH was still searching for an appropriate EHRS that meets their requirements and aspirations.

"The search for a suitable system is still underway." (HD 1)

"Up to this time, we have not implemented any appropriate systems to the PHCs." (Analyst 2)

"For the PHCs, an appropriate system has not yet been selected and we are still looking for a system that facilitates all activities and services in the PHCs." (Analyst 2)

In addition to software selection, training and awareness campaigns were discussed during the formulation of the plan. The following section will describe the plan to introduce the system through the awareness campaigns.

\subsubsection{Training}

As mentioned earlier, training will be provided side by side with the awareness campaigns. Therefore, the Saudi MoH has paid attention to the provision of training, 
setting up several training courses and developing a proper plan to provide training for all EHRS users.

"We are prepared to arrange suitable training programmes for users." (Analyst 1)

"Training is very important to ensure the success of the EHRS usage on an ongoing basis." (HD 1)

Moreover, the majority of participants agreed that training courses are essential to the EHRS implementation plan. For example:

"The training courses received much attention in the planning." (HD 1)

“Training is a key element in the planning." (DHD 1)

On the other hand, interviewees illustrated that training courses will be presents in two languages (Arabic and English) in order to be accessible to all end-users, even nonSaudis.

"The content of training materials will be printed in two languages, English and Arabic, for the convenience for all users." (GM1)

"Training courses will be presented in both Arabic and English languages." (DHD1)

\section{Training delivery methods}

Training courses will be implemented in a number of ways, including requesting the health affairs administration, in each region, to hold training courses for the EHRS users in that region. In addition, the $\mathrm{MoH}$ will distribute guide leaflets to help EHRS end-users to understand the system.

"Training will be centralised through directing the mission to the regions' administrations, where each health affairs management ensures to train their PHC staff." (HD 3)

"With the distribution of guided leaflets to make it easier for the user to use the system, they can educate themselves without the need for trainers." (Analyst 1) 
Another planned method by, the Saudi MoH, to provide training to all EHRS end-users is the concept of trainer training. One member from each PHC will be trained and then this member provides training to his/her colleagues at the same PHCs.

"We will train one employee who, in turn, will train another employee in the PHC.” (DHD 1)

"Train some users; after that the users will train new employees in the future." (Analyst 1)

\section{$\underline{\text { Training provision as a condition of the contract with vendors }}$}

The Saudi MoH is planning to include a clause in the contract with selected vendors to take the role of provide training courses for EHRS end-users.

"One of the contract terms put to the vendor is to train the users." (HD 1)

"Other options would be for the vendors to arrange and provide training courses for users.” (DHD 1)

\section{Training provision timeline}

As planned, the training sessions will be held in the early stages of the implementation of EHRS (pre-implementation phase).

"The training courses will be held in the pre-implementation stage." (GM 2)

“At the beginning of the project, training courses will be provided.” (HD 1)

Others stated that the training begins in the early stages of an EHRS implementation project and will last throughout all project phases, including the post-implementation phase.

"Training will be on an ongoing basis from the beginning of the implementation; even after the implementation." (GM 3)

"This will be ongoing during the implementation and after." (HD 2)

\subsubsection{Technical support}

In addition to training, technical support is another element discussed during the planning of EHRS implementation in PHCs. Technical support is one of the pillars of the project, 
and it is provided on an ongoing basis after the implementation of EHRS, especially in the post-implementation phase. In addition, the participants emphasised that technical support is provided from within SA and not from outside the country if the EHRS is implemented by an international vendor.

"Technical support is very important, and it is important to have technical support from within SA and not from the country we are buying the system from." (HD 1)

In the same context, technical support will be provided remotely through call centres in the headquarters of the MoH. However, this method is subject to the connectivity between the $\mathrm{MoH}$ and the PHCs.

"There is a huge call centre in the MoH to provide technical support. We are hoping to get the connectivity to provide remote support to the PHCs. " (Analyst 3)

\section{Provision of technical support as a condition of the contract with vendors}

As planned, similar to training, technical support will be provided by the vendors who implement the EHRS. Therefore, the Saudi MoH obliges the vendors to take responsibility for providing technical support on an ongoing basis, which will be included as a clause in the contract with the selected vendors.

"We will ask the companies to provide technical support constantly by adding some items to the contracts before signing." (HD 3)

"Technical support will be considered and approved during the agreement of any contract with any vendor." (HD 2)

In this context, technical support will be conducted on three levels, as follows: MoH level, represented by the call centre, service provider level, represented by the Telecom Communication Companies (TCCs), and vendor level.

"Usually, there will be three levels of technical support: 1) through the call centres at the Ministry; 2) services providers such as Saudi telecom companies;

3) through the vendor." (HD 3) 


\subsubsection{Security, privacy and confidentiality}

During the planning phase, the project team considers issues related to the protection of patient data, by taking several steps to improve security system standards in order to maintain patient confidentiality. Such steps were taken at both technical and human levels. Moreover, the Saudi MoH applied very strict rules and regulations to prevent any irregularities or breaches of patient data. The main procedures conducted by the $\mathrm{MoH}$ are: monitoring, implementing data protection law, applying non-disclosure agreements, giving privileges to users, and selecting secure systems.

"There is a big focus on security and protection of patient data as well as data confidentiality and privacy." (Analyst 1)

"There are policies that have been developed for security and confidentiality." (Analyst 3)

\section{Data protection laws to protect patient data}

The Saudi MoH has adopted laws to protect patient data from unauthorised access and any attempt to use it illegally. These laws involve deterrent punishments for any unethical use of such information.

"There are also systems and governmental laws concerned with punishing and preventing any illegal usage.” (GM 1)

"Strict standards and penalties for any use not in a moral position." (DHD 1)

Moreover, the MoH prompted all EHRS users to sign a "non-disclosure agreement", which contains clauses that ensure the protection of patient information from any misuse. In addition, the MoH sends a "confidentiality letter" to all EHRS users and vendors. Through this document, the user undertakes not to disclose any information concerning the patient or to use patient data for any purpose other than healthcare.

"The confidentiality of data is very important and, based on this matter, the Ministry in turn has prompted all its employees to sign a Non-Disclosure Agreement." (HD 1)

"A ratification has been made and should be signed by the vendors and the users themselves. It is called a Confidentiality Letter; the ratification contains a clause stating not to use any information for any other purposes." (HD 3) 


\section{Monitoring and auditing by $\mathrm{PHC}$ directors}

The Saudi MH is planning to monitor these systems by granting PHCs directors the authority to monitor all transactions made by PHC staff to detect any misuse during the use of these systems.

"The Ministry not only endorsed this ratification, but carries out audits and follow-ups of all electronic transactions. In the case of proven misuse, there is a penalty." (HD 3)

"We have given each centre director the power to monitor, so the director knows exactly what each employee does, how many orders have been entered and how many patients have been served." (SD 1)

\section{Limited access privileges}

All EHRS end-users from different levels and occupations will be provided limited access based on their role. Therefore, privileges will be granted to those users to avoid any unauthorised access.

“...privileges will be determined for each user.” (HD 1)

"In terms of users themselves, they will be given privileges based on each user's role and responsibilities.” (GM 1)

\section{Selecting a secure system}

In addition to the procedures mentioned above, system security will be taken into consideration during software selection.

"One of the Ministry's criteria for the selection of any EHRS is that the system should be secure and not penetrable. We will test any system before we purchase it." (HD 3)

The findings in this section show that the design of the EHRS plan took into consideration most of the factors that influence EHRS implementation, and illustrate in detail the role of consultation in implementation planning. The findings show that large-scale projects require further consultations conducted at three levels: country, organisational, and individual. Training will accompany the awareness campaigns and will be delivered via the train the trainers concept. In addition, training will be provided via the health affairs in each region. Technical support has also been described as an essential factor of the 
EHRS implementation plan. To ensure the provision of technical support, the project team included this aspect as a condition in the contract with the potential vendors. Finally, factors related to the security and confidentiality of patient data were also included in the EHRS implementation plan. As illustrated, a set of data protection laws have been adopted to ensure security and confidentiality, in addition to other procedures, such as providing privileges to end-users, monitoring transactions in the system, and selecting a secure system.

Factors related to the EHRS itself were highlighted in the implementation plan, such as systems interoperability and the development of such a standard. It has been described that interoperability will be addressed during the software selection to ensure the selected EHRS is compatible with other systems. On the other hand, as described, software selection will be made taking into account criteria such as efficiency, usability and security. All these criteria and other requirements were included in the RFP document which has been formulated by the project team. Once project plan and other preimplementation procedures described, I went to ask about the impact of CM and FR on EHRS implementation in PHCs. The following section will present participant responses related to the impact of these factors. 


\subsection{Theme Three: The role of Centralised Management (CM)}

The previous section describes the EHRS procedures that included in the planning phase. This section highlights the role of CM on EHRS implementation in PHCs in SA (see Chapter Two). As illustrated in Chapter Two, leadership and management have been found to be influential factors of EHRS implementation. However, the role of CM has not been examined previously, therefore, in the current study I examine its role in detail. The Saudi MoH utilises CM methods. The findings of this section show a consensus that $\mathrm{CM}$ has a positive impact on the implementation of EHRS in PHCs in SA. Some of the project team emphasised that $\mathrm{CM}$ is best suited to the nature of the management system in Saudi Arabia. Some of the participants stated that this type of management system was appropriate in SA due to the similarities of the functions and business processes in the PHCs. It was commented by some that decisions related to EHRS implementation should be made centrally, by the Saudi MoH, to enhance the success of the project.

"...particularly in SA, because the PHCs in SA are similar, are under the Ministry's management and all have the same functions.” (Analyst 2)

"In the SA, in particular, it is necessary to use CM to ensure project success." (SD 1)

However, others suggested that the utilisation of a semi-centralised management system is more effective for implementing a large-scale EHRS. They argued that the adoption of this type of management represents a major challenge to the Saudi MoH and would be more effective, if the $\mathrm{MoH}$ involved representatives from regional sub-departments in decision-making. So, decisions regarding EHRS implementation could be made in coordination with representatives from lower levels, such as health affairs' administrators in the regions or members of the PHCs.

"CM is a challenge for the Ministry represented by the IT department due to the scale of the project, and therefore must involve sub-departments, health affairs in regions and also PHCs." (HD 2)

"With regard to the CM, all projects should be under the supervision of a central administration in co-ordination with representatives of regional health affairs administration to implement the large-scale EHRS in all PHCs. Therefore, it is better to use semi-centralised management and maintain the central management 
at the same time with the support of representatives to coordinate with the regions." (Analyst 2)

It was also recommended that the health affairs' administration should provide the regions with more authority in regard to EHRS implementation projects.

"In the current situation, regional management should be given some authority and functions to facilitate the implementation of the EHRS in PHCs which cannot be linked to the Ministry through the web. In this case, it is better to use semicentral management, so they can implement orders and directives directed by the Ministry. Then pass on powers and authorities to the regional management to execute and coordinate in addition to monitoring and auditing by the central administration at the Ministry." (Analyst 2)

\section{The impact of CM on decision-making}

All participants agreed that CM has a positive impact on decision-making. For example:

“CM's role is very positive.” (GM 1)

“CM has a very positive impact on decision-making." (Analyst 3)

\section{The impact of CM on geographical challenges}

The geographical nature of SA is considered a challenge that may affect the success of EHRS implementation.

"Geographical nature was a challenge to the MoH." (HD2)

The concept of CM has been found to be useful to overcome this challenge. The majority of the project team agreed that $\mathrm{CM}$ has a positive impact on the geographical factor. For example:

"CM is the best." (SD 1)

“CM has a positive impact.” (Analyst 3)

Nevertheless, there was some disagreement; a head of department thought that CM has a negative impact on the geographical factor.

"I think the effect is negative." (HD3) 
On the other hand, one of the analysts believed that CM has no impact on the geographical factor.

“CM will not have a significant role." (Analysts 2)

\section{The impact of CM on EHRS interoperability}

Interoperability was another measured factor, found to be positively influenced by CM. $\mathrm{CM}$ facilitates communication among project teams to unify decision-making regarding a system that can be interoperable in the future. In addition, this contributes to making the process of communication between the systems more efficient and smoother, and minimises any issues related to future EHRS interoperability. Consequently, this matter will reflect positively on EHRS implementation.

"EHRS interoperability is crucial, it is highly affected by CM and cannot succeed unless CM is adopted. So, it has a very positive impact." (HD1)

"In general, CM has a positive effect through the selection of a single system for PHCs to facilitate communication with each other in the future." (GM2)

\section{The impact of CM on the scale of the EHRS implementation project}

There is collective agreement on the positive impact of $\mathrm{CM}$ in overcoming challenges, related to scale of the project. A general manager believes that the CM is "Certainly positive” (GM1). Other participants state, for example:

"It has a very positive effect." (HD 3)

"The CM system has a positive effect regarding the implementation of the EHRS in a large number of centres." (SD 1)

Firmly believing that $\mathrm{CM}$ has a positive impact on the number of PHCs, a proposal has been made to divide the PHCs into zones or regions to overcome any issues related to the scale of the project and facilitate the implementation process.

"It has a positive impact. I would probably recommend dividing them into groups of different regions or zones." (Analyst 3) 


\section{$\underline{\text { The impact of CM on planning EHRS implementation in PHCs in SA }}$}

The majority of the project team agreed that CM has a positive impact on planning for the implementation of an EHRS. For instance, Analyst 1 said CM has a "very positive effect", and GM3 stated that the impact of CM is "positive". One of the general managers illustrated that CM helps to avoid many of the technical and administrative problems that occur during the planning phase by standardising any decisions and opinions. In addition, $\mathrm{CM}$ helps to reduce the costs of the project, since the planning occurs centrally at the Saudi MoH.

"The PHCs in SA are similar, have the same characteristics, and are working in the same field, so, CM is considered the best and easiest way to standardise decisions and opinions as well as avoid many technical and administrative problems." (GM2)

"It is better to be centralised, because when the planning and application is centralised the cost of the project will be reduced." (GM2)

On the other hand, one of the analysts believed that CM has a negative impact on EHRS implementation planning.

"The CM has negative impact on the planning phase" (Analyst 3)

\section{The impact of CM on software selection}

According to the participants' responses, CM has a positive impact on the software selection process. As illustrated by one of the general managers, "CM is very positive, without a doubt" (GM1). In this context, software selection should be carried out centrally and under the supervision of the Saudi $\mathrm{MoH}$.

"CM is positive with regard to EHRS selection, which should be done under the supervision of the Ministry." (SD 1)

"Such a decision regarding the software selection should be centralised at the top management of the Ministry.” (HD 2) 
Likewise, one of the heads of department emphasised the importance of software selection being made centrally at the top level of the Saudi MoH. He believed that lower level organisations, such as regional health affairs, do not have the ability to select or even examine an EHRS due to a lack of IT expertise.

\section{"Before choosing a system, we must evaluate and review the EHRS and compare prices. These things cannot be done in health centres where there are no experts in IT." (HD 3)}

Although software selection should be carried out centrally by the $\mathrm{MoH}$ in order to be positive and effective, all project stakeholders need to be involved, and champions must be appointed to enhance decision-making regarding software selection. Therefore, software selection should be made by representatives of the beneficiaries of the software to obtain their opinions and requirements.

"Choosing the appropriate system must be done through CM and in co-ordination with concerned parties, taking in their opinions and requirements; all beneficiaries of the system should participate." (Analyst 2)

"That will be done centrally, but again the impact depends on how you get people involved, whether you have change champions, and how you make everybody happy with the software selection." (Analyst 3)

On the other hand, due to previous experience in similar EHRS implementation projects, one of the analysts claims to have adopted the concept of a semi-centralised management to enhance software selection.

"From the experience of previous implementation projects, there were many notes from regions about the system, so health affairs in the regions should participate in system selection." (Analyst 2)

\section{The impact of CM on team communication processes}

There was a complete consensus that $\mathrm{CM}$ has a positive impact on team communication. For instance, HD 2 said "its effect was positive". As illustrated by some of the participants, project team communications were made at the headquarter in the Saudi $\mathrm{MoH}$ in the form of meetings and workshops. All such meetings and workshops also involve the project team at different departments and different regions. This greatly facilitates the decision-making process, reducing the time spent on it. 
“...CM is best to ensure communication between the project team.” (Analyst 2)

"We were holding meetings in the Ministry. The meetings were held centrally at Ministry level. The Ministry calls some employees from the regions to participate in these meetings and workshops. Each region was sending representatives from their area, so certainly it has a positive impact." (GM 1)

\section{The impact of CM on project team selection}

Team selection is also positively affected by CM. according to one the general managers, team selection is easier and more efficient when conducted centrally by the Saudi MoH. According to GM2, "CM is the best" in terms of enhancing project selection, and DHD1 said "It has a very positive impact".

“...CM dramatically facilitates this process because project team selection is easier and of more benefit." (GM 1)

This section evaluates the impact of CM on EHRS implementation in PHCs in SA. The findings of this section show that CM has a positive impact on EHRS implementation in PHCs in SA. Decision-making, EHRS interoperability, project management and leadership, project team communication and selection were found to be the factors most affected by CM. While, planning and scale of the project were found to be less influenced by $\mathrm{CM}$. The following section presents findings related to the impact of FR on the implementation of EHRS in Saudi PHCs 


\subsection{Theme Four: The role of Financial Resources $(F R)$}

This section highlights the role of FR in EHRS implementation in PHCs in SA (see Chapter Two). The Saudi MoH is characterised by an abundance of financial resources provided by the Saudi government, and the participants agreed upon this unanimously. Overall, FR has a very positive impact on EHRS implementation projects. All the participants reported that financial resources contributed positively and facilitated the success of many previous projects, in particular EHRS implementation projects, due to the country's ability to fund electronic transformation in all sectors and services. For instance, the participants said:

"The role of FR is definitely positive; this country has more access to financial resources." (Analyst 3)

"Positive, without a doubt." (HD 1)

"FR is very positive." (HD 3)

"The financial resources are the most important factor that contribute to the success of the project." (Analyst 1)

"The main factor which helps us to implement EHRS is financial support." (SD 1)

Furthermore, a head of department stated that "there are no financial problems that are hindering the implementation of the EHRS" (HD1), and one of the general managers said "the Kingdom does not suffer at all from the problem of availability of financial resources as they are available; plentifully and thankfully." (GM 1)

The provision of FR assists in the accomplishment of EHRS implementation projects in general. The Ministry had to fulfil all the decision-makers' requirements to complete the EHRS implementation project regardless of the cost, and was committed to providing them.

"We have a high budget for the implementation of the EHRS both in hospitals and health centres.” (GM 1) 
"Yes, we received an adequate budget for this project. It was supposed to provide a very strong balance sheet." (HD 3)

In regard to overcoming technical and other organisational challenges, the software developer revealed that a sufficient budget had been allocated for specific elements such as technical infrastructure and training.

“We received major financial support to set up the infrastructure.” (SD 1)

"The Ministry appropriately financed the courses and provided housing and transportation for all trainees when they had to travel to attend courses at the Ministry." (SD 1)

Three percent of the Ministry's annual budget is allocated to the implementation of EHRS projects. This is stipulated in the policy of Saudi Arabia, where the same proportion of the budget of any ministry is allocated to information technology. In the case of the $\mathrm{MoH}$, this amount is approximately three billion Saudi Arabian Riyals, which is equivalent to six hundred million pounds.

"After developing the strategic plan of the Ministry, it was approved by the Council of Ministers who allocated three billion riyals (6 hundred million pounds) for adoption of IT in the MoH. It was the biggest budget ever for the Ministry to support IT implementation, and the support is still ongoing. The state policy has allocated $3 \%$ of the budget for any ministry for IT projects. This is very significant support and the figure was adopted annually, will cover all the costs of IT and will certainly facilitate the implementation of EHRS overall.” (DHD 1)

\section{The impact of financial resources on the provision of hardware}

All participants reported that the abundance of financial resources positively affects the availability of efficient, high-performance computers and other devices needed to run the EHRS without any issues.

\footnotetext{
"Positive, one hundred percent." (Analyst 1)

“...the availability of financial resources helps in providing high efficiency devices to ensure the system is running without any problems.” (GM 1)
}

However, one of the analysts reported that the provision of hardware wasn't among the challenges of EHRS implementation in PHCs. 
"I don't think the provision of appropriate hardware is as big a problem as you think." (Analyst 3)

\section{The impact of financial resources on technical infrastructure}

There was unanimous agreement that technical infrastructure is positively affected by an abundance of financial resources. A general manager stated that the abundance of financial resources has contributed greatly to overcoming all the challenges associated with development of the infrastructure, such as connectivity for new projects. Developing an appropriate infrastructure is very expensive, so abundant financial resources contribute to its facilitation.

"FR helped us to overcome the obstacles we encountered with regard to infrastructure. Infrastructure is the toughest obstacle which we have encountered, but with money availability it became easy to overcome." (GM 1)

“FR helps to provide the appropriate connection." (GM 2)

"FR has a very positive impact, because preparing infrastructure is very expensive." (Analyst 1)

\section{The impact of financial resources on systems interoperability}

One of the analysts argued that "FR has no impact" (Analyst 3). In addition, a software developer said, "I do not think that financial support has an effect on EHRS interoperability" (SD 1). However, the majority of participants confirmed that FR does have a positive impact on interoperability. FR assists in purchasing an effective standard, such as HL7. Moreover, FR allows organisations to select vendors that provide an interoperable EHRS.

"FR helps in the purchase of a standard such as HL7.” (GM 2)

"The abundance of FR gives you the option of selecting appropriate vendors which can provide compatible systems and not be limited to less expensive companies whose systems may not be compatible with other EHRS." (DHD 1)

"FR has a positive effect by providing high quality standards and making EHRS compatible with each other." (Analyst 1) 


\section{The impact of financial resources on $\mathrm{PHC}$ restructuring and workflow redesign}

The majority of respondents stated that FR has a positive influence on restructuring and workflow design. For instance, GM2 said “FR helps to redesign the PHC workflow”. The Saudi MoH was able to sign a contract with a specialist company to assist them during the restructuring of the PHCs to be ready for the new EHRS implementation.

"FR helps to sign a contract with consulting firms specialising in business reengineering." (DHD 1)

However, others believe that FR has limited and even no impact on PHC restructuring and workflow redesign.

"Very limited impact on this matter, but it is a positive." (HD2)

"This matter has nothing to do with FR, from my point of view." (Analyst 1)

\section{The impact of financial resources on software selection}

All participants agreed that FR has a very positive impact on the selection of an appropriate and highly efficient EHRS. FR facilitate the selection of the most efficient system. For example:

"Definitely has a positive impact." (Analyst 3)

"Abundant FR are positive, as in such case the Ministry selects an excellent system." (Analyst 1)

"We have the option to choose the best system available, so it is very positive." (DHD 1)

Furthermore, software selection is highly influenced by FR. Thus, the $\mathrm{MoH}$ is not restricted to certain systems due to a lack of financial resources.

"Software selection is highly affected by FR, where it gave us a big chance to choose any EHRS without being confined to specific systems due to shortage in FR." (HD 3) 


\section{The impact of financial resources on project team communication}

The majority of the statements were in agreement with the positive impact of FR on project team communication. Project team communication is one of the costliest procedures, and FR contribute to overcoming this challenge, especially when the Saudi $\mathrm{MoH}$ holds meetings between members from different regions.

"Joint collaborative work between project team members in different regions is very expensive, and the money was available and helped us a lot." (GM 1)

"FR has a positive effect here, it was easy for us to hold meetings with all members from different regions, we also arranged visits to the PHCs for consultation and evaluation. FR were always available for these activities." (HD 3)

\section{The impact of financial resources on project team selection}

The participants agreed that there are professional shortages in HI and IT. However, FR assisted the Saudi $\mathrm{MoH}$ in attracting qualified personnel to participate in the EHRS implementation project. Thus, FR are considered to have a positive impact on team selection.

"It is positive. The formation of an excellent project team, large amounts of money paid to hire experts." (HD 1)

"The availability of FR assists in hiring talents and experts, otherwise we would have had big problems and select unqualified people, which may affect the implementation of such large-scale projects." (GM 1)

"The financial support has had a very positive impact on the provision of experts." (Analyst 2)

However, others argued that IT and HI professional's availability is a worldwide issue and FR have no impact on the provision of those individuals.

"It is not a cost issue, it is a worldwide shortage, so I don't think FR has a role." (Analyst 3) 
"In terms of expertise and competencies, I do not think FR has an effect." (GM 3)

\section{The impact of financial resources on the provision of technical support}

Once again, the participants unanimously agreed on the positive impact of an abundance of FR on the provision of technical support. The provision of technical support is very costly. Thus, with sufficient money, fifteen to twenty percent can be added to the cost of the contract fees for continuous technical support, maintenance and insurance. Thus, the Saudi MoH can ensure the success of the EHRS implementation project. Moreover, FR contribute positively by paying for technical support twenty-four hours per day, with the $\mathrm{MoH}$ compensating the technicians for excessive working hours.

"FR are positive because if we buy the EHRS we can add $18 \%$ or $20 \%$ to the project cost to provide continuous technical support as well as insurance and system maintenance." (GM 1)

"It has a positive effect because technical support should be continuous, thus extra pay for additional work hours or more technicians is necessary, and this requires providing large amounts." (Analyst 1 )

\section{The impact of financial resources on the provision of training}

None of the participants reported that the abundance of FR has a negative or neutral impact on the training process. All agreed that an abundance of FR has a positive impact on the training process. For example, Analyst 3 said "Absolutely, FR has a positive impact" on provision of training, HD1 said, "It has a very positive effect." Analyst 2 said, "FR is essential and has a very positive impact, particularly on training."

This section evaluates the impact of FR on EHRS implementation in PHCs in SA. The findings of this section show that FR has a very positive impact on preparation of infrastructure, software selection, technical support, project team communication and provision of training. Project team selection, restructuring and workflow redesign were found to be less influenced by FR. Furthermore, I asked the interviewees about the facilitators and barriers to EHRS implementation in PHCs in SA. The participants responses will be presented in detail in the following section. 


\subsection{Theme Five: Facilitators and barriers to EHRS implementation}

This theme includes all generated codes which represent barriers and facilitators to EHRS implementation in PHCs in SA. Several barriers and facilitators were mentioned by the participants, however, the number of the identified barriers is greater compared with the facilitators of EHRS implementation. Moreover, several steps to overcoming the barriers that may lead to implementation failure were reported. This section will be divided into three sub-themes: codes related to the facilitator, codes related to barriers, and steps taken to overcome the barriers.

\subsubsection{Facilitators of EHRS implementation in PHCs in SA}

A number of factors assisted the project team during EHRS implementation in PHCs in SA. These facilitators include e-government, the characteristics of PHCs in SA, financial resources (see Section 7.5), leadership and management, user willingness, and perceived usefulness of the EHRS.

\section{$\underline{\text { E-government trend in SA }}$}

As stated by a general manager, the Saudi MoH has been encouraged by the government to move toward digital transformation. This trend is supported directly by the higher authority in SA, which is also highlighted in the Saudi Vision 2030 (see Chapter One).

"The main orientation of SA is transforming all government services into electronic transactions. This is an important factor which helped and encouraged the Ministry to implement EHRS in all sectors." (GM 1)

\section{Characteristics of PHCs}

PHCs in SA are very similar to each other in terms of the healthcare provided and business workflow. The business process, structure and workflow are considered to be the same in all PHCs in SA. This facilitates software selection and other processes of EHRS implementation.

"If you have a look at the PHCs, they all offer the same services and the same standards; there is no difference between them." (HD 1) 
"The PHCs in the Kingdom are similar, have the same characteristics and work in the same field." (GM 2)

Moreover, PHCs in SA are distinguished by their small size and number of staff compared to other healthcare sectors in SA. The small size of Saudi PHCs allows greater flexibility to implement new projects and assists in overcoming challenges such as training.

"The small size and number of staff in PHCs is ideal for the training process. Services provided by the PHCs are easy when compared with hospital services, and that helps in the implementation of the system and training." (HD3)

\section{$\underline{\text { Leadership and management }}$}

Management and leadership have been recorded as instrumental in the success of an EHRS implementation project.

"Management and leadership affect us positively and they have a very significant role in contributing to facilitating our mission." (SD 1)

"Leadership and management have an important role and are essential to the success of any project. The most important thing that affects such projects is the support from leaders and managers." (Analyst 1)

In this context, strong leadership has a very positive impact on EHRS implementation projects and contributes significantly to the success of any project, particularly when support comes from senior management and others who have authority and influence at the ministry level. Concurring with this view, one of the general managers claimed that the success of EHRS implementation projects is fifty percent dependent on strong support and leadership at the senior manager level. Due to its importance and great influence on the success or failure of EHRS implementation projects, leadership has been discussed at all conferences, meetings and workshops held at the Saudi MoH.

"Support from senior management is one of the most important facilitators of successful EHRS implementation.” (HD 1)

"EHRS implementation projects generally rely $50 \%$ on management and leadership.” (GM 1)

"Regarding management factors, leadership in such projects is one of the most important factors contributing to a systems' implementation; this matter has 
been discussed in all the conferences, workshops and meetings I have attended." (HD 3)

One of the benefits of strong leadership in EHRS implementation projects is that it ensures there is no disruption or delay. In addition, it prevents complacency in relation to the completion of these projects and minimises any errors; as represented in the comment below:

"It certainly helps in the success of the system by providing adequate budgets, careful follow-ups and supervision, and gives strong commands to ensure that there is no leniency or delay in the implementation of any project." (HD 3)

Furthermore, the participants highlighted the positive role played by senior managers in driving the development wheel, especially in relation to participating in the implementation of an EHRS in PHCs. HD2 said, "No doubt, the senior management team are the foundation, and if they don't involve, the project may fail'. The highest authority in the Ministry, represented by the Minister and his deputies, were involved in the EHRS implementation project.

"There was high-level participation of the Ministry in this project, and they have a big role in this project." (Analyst 2)

The participants reported that two categories of support are provided by the senior managers: moral and financial, and also talked about the significance of the power of management and leadership in the success of EHRS implementation projects.

Analyst 1 stated that, "the most important thing that affects such EHRS implementation projects is the support from top management, whether moral or financial". Therefore, any project included in the e-health strategy has received great moral or financial support from managers at the Saudi $\mathrm{MoH}$, including support from the highest authority at the Ministry. Moral support was provided by seniors and top management at the Saudi MoH. Seniors were directly involved in the project to provide recommendation and to overcome challenges faces the project team, such as delay. 
"All projects within the Ministry's e-health strategy have always received both moral and material support from top management at the MoH". (DHD 1)Perceived usefulness of an EHRS

The saving time and effort, cost and error reduction and disease control benefits of an EHRS were considered as the most important factors that encourage senior managers to implement it.

"Senior management became aware of the role of IT and the extent of savings that could be achieved with the EHRS, whether in terms of money, time or effort. Senior management are also aware that the EHRS will help to reduce errors." (GM 3)

\section{EHRS end-user willingness to use the system}

End-user willingness plays an important role in EHRS implementation in Saudi PHCs.

"One of the main facilitators is the willingness in users themselves." (DHD1)

This section determines facilitators of EHRS implementation in PHCs in SA. Several facilitators have been detected in the findings presented by this section. The Saudi MoH has benefited from support from the Saudi government. Moreover, the small size and the unified business process of all PHCs also facilitates the EHRS implementation project. Moreover, the project team benefited from abundant financial support, where a sufficient budget was allocated to help accomplish the EHRS implementation project. Strong leadership and follow-up from the highest authority in the Ministry assisted in overcoming many challenges. Finally, the project team benefited from the willingness of end-users to introduce the EHRS in their workplace.

\subsubsection{Barriers to EHRS implementation in PHCs in SA}

There are many obstacles to the implementation of EHRS in PHCs.

\section{"The obstacles are too many." (SD1)}

Through the interviews, the participants mentioned many obstacles that they had encountered during their involvement in EHRS implementation in PHCs. Barriers were represented by changing of staff, a lack of $\mathrm{HI}$ experts, lack of training, a large number of 
PHCs, implementation time, barriers related to vendors, a lack of connectivity, poor infrastructure, geographical challenges, EHRS interoperability, software selection, and a lack of appropriate computers.

\section{Changing people}

Changing individuals, especially at the level of policymakers or senior managers, has a negative impact on EHRS implementation projects. Each new minister arrives with a new plan that cancel all that had been done previously, both in terms of plans and adopted strategies. Changes were not confined to plans and strategies, but also included changing the administrative staff and decision-makers.

"Every minister cancels the previous plan and develops new plans and strategies." (GM 2)

"Unfortunately, we have a problem in SA in that new ministers remove the plans and decisions of the former minister." (DHD 1).

Due to these changes, obstructions and delays have marred the execution of many EHRS implementation projects.

"Some obstructions, delays and execution of works are also affected by constant changes that happen in the Ministry, including replacement of the Minister and some seniors in the MoH." (GM 1)

"Unfortunately, frequent changes at the ministerial level and the subsequent changes at the departmental level had a negative effect on the completion of the projects." (GM 2)

The consequences of these changes are not only delays and disruptions to the projects, but also the termination of existing projects (EHRS implementation in PHCs in SA).

"Unfortunately, with new seniors and a new Minister, the work on the current project stopped.” (HD 3)

Similarly, changes at staff level in PHCs were found to have a negative impact on EHRS implementation, particularly the provision of training. 
"Another problem that has been encountered is that of staff change. Many times, after three or four months of training one employee, another employee comes instead of that one." (SD1)

\section{Shortage of expertise in EHRS implementation}

The Saudi MoH suffers from a shortage of expert staff, such as IT technicians and HI professionals.

"One of biggest challenges is finding talented people in specific areas, especially in SA." (GM1)

"Finding efficiencies is a very difficult task; we also have a very big problem with labour availability." (HD1)

\section{Lack of training}

Training is another challenge the Saudi MoH faces, particularly with regards to the use and implementation of EHRS in PHCs. The majority of the study participants agreed that training was one of the main barriers to EHRS implementation. For example:

"Most obstacles with regards to employees/users are associated with training." (HD 1)

“The lack of training is among the problems.” (HD 1)

"Of the main obstacles is training." (Analyst 1)

\section{Scale of the project}

The number of PHCs is very large and represents an obstacle to the implementation of EHRS. The delivery of training and technical support is negatively influenced by the scale of the project.

"The biggest problem is how to install the EHRS into more than 2,000 PHCs, so it is a huge issue because it is equally important as the hospitals." (Analyst 3)

"The main obstacles are training and technical support in particular; with regards to the PHCs, problems arise due to the large number of the PHCs." (Analyst 1) 


\section{Timeframe for implementing EHRS in PHCs}

The time required to implement a large-scale project was one of the challenges that faced the project team, as large-scale projects require more time and may cause delays.

"Our problems are often associated with time, as the implementation of such large projects requires a lot of time." (HD3)

"We also have difficulties such as delays in large-scale projects such as the current one." (GM 1)

\section{Geographical challenges}

The size and geographical nature of SA is considered to be a major challenge by the Saudi $\mathrm{MoH}$.

"Geographical nature is a challenge for the MoH." (HD2)

"The geographical nature of the Kingdom is considered to be a big challenge to EHRS implementation." (HD 3)

The location of some PHCs is one of the factors that make the Kingdom's geography a major obstacle to the implementation of EHRS. In particular, those located in rural or remote areas.

"The geographical nature constitutes a challenge because there are many PHCs located in remote areas." (DHD 1)

"The most influential obstacles to the MoH are PHCs which are located in remote areas." (GM 3 )

\section{Inadequate infrastructure and lack of connectivity}

The geographical challenges described above have a direct relationship with infrastructure. Hence, infrastructure and connectivity are another two major challenges to EHRS implementation in Saudi PHCs. Connectivity between PHCs is considered a dilemma by the Saudi $\mathrm{MoH}$ and has led to the delay of many projects. Lack of connectivity between PHCs is a result of poor infrastructure.

"The challenges are still the same, especially in terms of connectivity and infrastructure." (Analyst 3) 
"Also, we faced other difficulties related to the infrastructure such as connectivity, especially with PHCs in remote areas." (GM 1)

"We have talked about many of the barriers; especially regarding infrastructure." (HD 1)

"The development of infrastructure for each PHC is considered a major obstacle to the implementation of the EHRS." (DHD 1)

"Connectivity is considered one of the main barriers." (GM1)

"The technical side is a very important factor regarding the development of the infrastructure and networks, it is possible to choose the best system in the world, but when it comes to implementation, the surprise is that the infrastructure may not be suitable for implementation." (Analyst 1)

Developing a proper infrastructure can be very expensive, especially since some of the PHCs are operated from rented properties which are not suitable for such IT projects.

"Infrastructure involves a very expensive process of communication between the health centres, especially since some of the PHCs are in rented buildings." (GM 3)

Lack of hardware, such as computers, in some of the PHCs is another barrier related to infrastructure.

"Lack of computers is one of the obstacles we faced at the beginning, especially in non-developed PHCs." (Analyst 2)

\section{$\underline{\text { Software selection }}$}

Lack of an appropriate EHRS that meets project team ambitions and aspirations was highlighted as another barrier to EHRS implementation in Saudi PHCs. The majority of the EHRS that were presented to the Saudi $\mathrm{MoH}$, in particular international systems, do not correspond with the characteristics and functions of the PHCs in SA.

"The most difficult obstacle is that until now we haven't found an appropriate EHRS that can achieve our needs and satisfy our ambition." (Analyst 2)

"We also face a big challenge, we cannot find either a local or global EHRS that meets the requirements of PHCs in SA, and the most important reason is 
that there are not many options for PHCs because all global companies focus on hospitals and making systems that fit into hospitals." (HD 3)

The nature of the PHCs in SA is different from that of PHCs in other countries such as the UK, USA and Australia. These differences have hampered software selection due to lack of a highly efficient world-class EHRS that fits the current functions of PHCs in SA.

"... and what made it even more difficult is the differences in the characteristics of PHCs and business workflow in SA compared to that of large countries such as America, Britain and Australia. They apply so-called 'GPs' rather than PHCs." (HD 3)

"We did not find an EHRS that is compatible with the workflow of the PHCs in SA.” (GM 3)

"We received offers from outside the Ministry from international companies who had previous experience in the implementation of EHRS, but we faced a problem with these systems. They only suit the processes in other countries such as Britain, America and Canada." (GM 3)

Vendor selection is another obstacle to the implementation of EHRS for several reasons, including: vendors are inefficient, increase their prices, and do not have sufficient experience in the Saudi healthcare system. These three factors represent obstacles to the selection of suitable vendors to entrust with EHRS implementation projects. A lack of sufficient experience saw the project team hesitate to make a decision in this regard. Moreover, exaggerated prices made it difficult for the Saudi MoH to take further steps to sign an agreement with vendors. Finally, some of the vendors were not efficient enough to satisfy the ambitions of the project team at the $\mathrm{MoH}$; as indicated by the quotes below:

"International companies have never implemented EHRS Saudi PHCs; this has made us hesitant to select international companies. " (GM 3)

"The drawback is the greed of vendors. I noticed that some big IT companies have raised their prices twofold, and even some prices three times. This has affected some of the decisions that have been made." (DHD 1)

"The main obstacles are contracting with a qualified vendor.” (SD 1) 


\section{Lack of EHRS interoperability}

EHRS interoperability has been determined to be one of the main barriers to the implementation of EHRS projects in PHCs in SA.

"One of the big challenges here is EHRS interoperability." (Analyst 3)

“We should take EHRS interoperability issues seriously.” (SD 1)

Similar to the previous section, this section determines the barriers to EHRS implementation in PHCs in SA. Several barriers have been determined to influence EHRS implementation in PHCs, including the changing of key people such as senior managers, particularly those directly involved in the project. Moreover, a lack of experts in the field of health informatics has been found to have an impact. The provision of training has also been recorded to be a barrier, as well as the large number of the PHCs in SA.

Moreover, technical barriers also inhibit EHRS implementation in PHCs in SA. In this regard, connectivity between PHCs was another barrier faced by the project teams, as well as poor PHC infrastructure. These barriers are influenced by the geographical challenges of SA. The selection of an appropriate EHRS and vendor was recorded as another barrier to the accomplishment of this project. In the same context, EHRS interoperability was deemed a technical barrier to EHRS implementation, as well as the availability of appropriate hardware.

\subsubsection{Overcoming barriers}

The Saudi MoH has taken a number of steps to overcoming the above-mentioned barriers and enhancing the success of EHRS implementation. Steps taken to overcome the above obstacles and challenges include developing the necessary infrastructure and standards, conducting research and studies (see Section 7.2.6), cooperating with Telecommunication Companies (TCs), selecting more than one vendor, dividing the Kingdom into regions, and involving all stakeholders. 


\section{Technology development}

It was noted that a key step taken by the Saudi MoH to overcome obstacles and challenges in EHRS implementation projects was the development of the current infrastructure, standards and other technical aspects.

"The key success, of course, is the development of standards and infrastructure." (Analyst 3)

The MoH also plan to develop the existing EHRS to comply with the aspirations of the $\mathrm{MoH}$ and users.

"The Ministry is currently studying the possibility of the development of the previous EHRS to be generalised and implemented in all PHCs." (HD 1)

\section{Dividing the country into regions}

As previously mentioned, the large scale of the project makes implementing the EHRS in PHCs very complicated. In an attempt to overcome this obstacle, the MoH divided the SA into five zones, and will build a data centre in each of these regions; all PHCs and hospitals, in each region, will be linked to each other via these data centres.

"As I mentioned to you earlier, the plan was to set up a data centre in each region after the division of the Kingdom into five regions (zones), to link the PHCs with these data centres, and then connect the PHCs with the hospitals." (HD 3)

"We divided the Kingdom into five zones; each zone will have one data centre." (GM 1)

"The Kingdom will be divided into five zones, data centres will be connected to PHCs, and then the hospitals shall be connected to the healthcare centres." (DHD 1)

Subsequently, more than one vendor will be selected to implement the EHRS to reduce the workload and minimise the risks.

"We will be contracting with at least three providers to reduces the pressure on the provider. If it is one vendor, the number to cope with is huge, and one company alone cannot implement the EHRS in all PHCs in SA." (GM 1) 


\section{$\underline{\text { Co-operation with Telecommunications Companies (TCs) }}$}

Another solution that may contribute to overcoming the obstacles is collaboration with communications and information technology companies. Indeed, the Saudi $\mathrm{MoH}$ has signed contracts with several communication and IT companies. The purpose of this collaboration is to overcome the geographical challenges and associated obstacles pertaining to infrastructure.

"The geographical challenges will be addressed through co-ordination with the TCs and connection to the Internet for all PHCs; then linking them to the data centres in each region." (HD 1)

"Contracts have been made with IT service providers." (DHD 1)

"Working in co-ordination with the TCs on the development of infrastructure." (Analyst 1)

\section{Piloting the system}

The Saudi MoH will run a pilot of the selected system by implementing the EHRS in a small number of PHCs. Thereafter, the $\mathrm{MoH}$ will evaluate the system to find out if there are any issues related to usability or any technical issues prior to actual implementation.

"First, we will select a system and try to implement it in some PHCs for the evaluation of several aspects to measure the system's usability and determine any problems; then we will collect and analyse the problems and solve them. We will work on this more than once until we achieve 100\% user satisfaction." (GM 3)

"The Ministry has developed a unified plan at all PHCs and areas. A number of centres have been identified to apply the EHRS according to priority in order to test the system at the beginning; these centres were ready to implement the system as they have an excellent infrastructure, so a number of centres have been selected in each region in the Kingdom." (SD1)

This section determines the procedures the Saudi $\mathrm{MoH}$ is to follow to overcome the mentioned barriers and challenges. The first step is to develop technical infrastructure and standards to assure system interoperability. In addition, the $\mathrm{MoH}$ is planning to conduct research and studies to enhance the implementation of the EHRS in PHCs. 
Moreover, the MoH has plans to divide SA into five regions to improve connectivity between PHCs. Data centres will be built in each region to link PHCs with each other and hospitals. In addition, more than one vendor will be selected. To enhance the connectivity between the PHCs, the MoH will coordinate with Saudi TCs. On the other hand, to avoid unexpected issues, the MoH will pilot the EHRS before it is fully implemented. 


\subsection{Theme Six: The implemented EHRS}

The Saudi MoH has previously implemented an EHRS in approximately 150 PHCs.

"I think it is 150 PHCs." (GM 1)

"It was implemented in 150 PHCs ten years ago." (GM 2)

However, this project failed due to several factors such as changes at the administrative level, particularly at the level of ministers and senior managers (see Section 7.6.2).

"Unfortunately, with administrative changes and the change of the former minister, work on the current EHRS was terminated in 2009." (HD 3)

\subsubsection{Evaluation of the implemented EHRS}

The Saudi MoH IT department evaluated the implemented EHRS on a regular basis.

“We were doing an assessment of the system on a regular basis.” (GM2)

"It was being evaluated from time to time." (HD2)

"The EHRS was evaluated by colleagues in the IT department.” (HD 1)

The evaluation process is performed using two different methods. The first method involves sending a team from the $\mathrm{MoH}$ to test the system on site, while the second method involves holding meetings with EHRS end-users and asking them to fill in a questionnaire to ascertain their views on the system.

"One of the assessment methods was sending teams to the PHCs to test the EHRS and stand on its problems." (GM2)

"I was holding a meeting with the end-users themselves and asked them to fill in a questionnaire.” (DHD1) 
However, others argued that no evaluation of the implemented EHRS had been performed.

“No evaluation has been done.” (Analyst 2)

"No assessment of the implemented EHRS has been done." (HD3)

\subsubsection{Perceived usefulness of the implemented EHRS}

Although the previously implemented EHRS has been considered a failure, it does have a positive impact on PHCs. Thus, the performance of PHCs has changed for the better, which has resulted in a major improvement in services provided to patients.

"There was a positive change detected, and there was a development in the performance of the PHCs and services provided. So, PHCs performance dramatically changed for the better with the utilisation of the EHRS. " (Analyst 2)

“Undoubtedly the EHRS changed the PHCs for better." (HD 2)

"The EHRS changed positively because it raised the level of performance and efficiency." (DHD 1)

Moreover, the performance of the staff in these PHCs has been positively affected. In addition, experience with using an EHRS has motivated those users to implement a new EHRS after the termination of the previous system. The former EHRS assisted in making users more aware of modern technology and to adapt to the needs of the future.

\footnotetext{
"The system made changes to user performance for the better." (Analyst 1)

"It created a desire among users to implement the new EHRS, and they are motivated to use the new system." (DHD 1)
}

\subsubsection{Technical support}

Technical support was considered one of the main challenges that the previous EHRS project faced. The Saudi MoH received numerous complaints from EHRS end-users due to the lack of technical support.

"We faced a problem with maintenance, many users complained of lack of maintenance of the system.” (GM2) 
Moreover, lack of technical support resulted in disappointment for many EHRS endusers and led to them being resistant to the system.

"Technical support was a source of concern for users. Among the problems I remember, therefore, we faced some opposition from the end-users, they were not willing to use the system due to lack of technical support." (DHD 1).

One of the general managers argued that lack of technical support was one of the causes that led to the failure of the previous EHRS.

“The EHRS failed because it was without technical support.” (GM 3)

Some of the general managers attributed the failure to provide appropriate technical support to two important factors, namely the lack of IT professionals in the regions to provide instant support, and not being able to provide technical support remotely, which requires technical support technicians to travel in person to the site.

"We faced problems with technical support; the EHRS malfunctioned for days due to a lack of IT technicians in the region." (GM 1)

"The reason is the difficulty accessing the PHCs because the system is not connected with the Ministry for easy maintenance by providing technical support remotely." (GM 2)

\subsubsection{Training}

When examining the provision of training to end-users of the implemented EHRS, some participants agreed that all EHRS end-users have received adequate training since the first day of implementation. For example:

“There was a very adequate training course.” (HD3)

"Staff were trained from the first day the EHRS was implemented." (SD1)

Moreover, training given was accredited by the Saudi MoH. The training sessions took place at different times and at different locations. It was noted that the training courses were carried out both inside and outside working hours as well as inside and outside PHCs. In addition, continuous training was provided to the PHC staff. 
"Training courses are held continuously, as needed; and courses are provided inside and outside the centres." (DHD 1)

"We had continuous training courses which were held outside work times and within work times.” (GM 2)

However, others argued that not enough training courses were provided for the EHRS end-users.

"There are not sufficient courses at the present time; they are few." (HD 1)

On the other hand, others stated that there are no training courses; training has been suspended for the former EHRS.

"At present, there is no training." (Analyst 1)

"Currently, the system is disrupted, and training sessions have stopped as well." $(S D$ 1)

\subsubsection{User awareness}

EHRS end-users were fully aware of the EHRS implementation project. At the beginning of the project, there was an awareness campaign conducted, via distributing brochures and sending announcements and circulars to the targeted PHCs. These contained information on the benefits of the system and its role in facilitating their tasks and improving the healthcare provided. In addition, the brochures contained a simple explanation of how to use the system.

"We made announcements and circulars stating that we will develop a system to be implemented in the PHCs. It also stated that the role of the EHRS will assist the users in providing high quality services to patients from the time they enter the PHC until they are released. We have also distributed posters and brochures." (SD 1)

\subsubsection{User resistance}

User acceptance varies from one PHC to another.

"The level of system acceptance varies from one PHC to another; some centres were enthusiastic and willing to apply it, whereas other centres have no desire." (SD 1) 
Some of the interviewees stated that the Saudi MoH had faced difficulties associated with the end-users themselves, as some of them were unwilling to use the EHRS. This is a major obstacle which may lead to implementation failure.

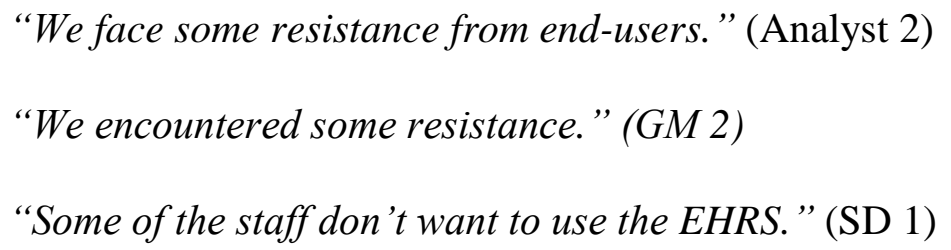

There are several reasons for reluctance to use an EHRS. According to SD1, one of these is computer illiteracy; it was found that users who do not have experience in using computers are unwilling to use the system. Another reason is that the PHC directors themselves did not seem willing to provide any support.

"We face some problems from users who have no computer experience - they do not want the system." (SD 1)

"We don't have appropriate support from the PHCs that we implemented the system in; especially the centres' directors. We didn't get the expected support from them." (SD1)

HD2 argued that the most likely reason for the end-user's resistance and unwillingness to use the system was due to the lack of full familiarity with EHRS and a lack of awareness of the purpose of their implementation in PHCs. Therefore, this participant provided a solution to overcome this issue, stating, "this problem is solved through training".

"In some cases, the lack of desire to use the system was caused by lack of understanding of how to use the EHRS.” (HD2)

Another reason was mentioned by Analyst 2, who perceived that elderly users are often more resistant. This is not confined to the use of EHRS, but also to the use of computers in the workplace in general.

"Many users, especially the elderly, do not want to deal with computers at work." (Analyst 2)

It has been illustrated that end-users may not be willing to use an EHRS in their workplace and may refuse the change. They believe that the new system may negatively affect their work routine and may even lead to them losing their jobs. 
"It is very natural to find a person who doesn't want to change; most of the employees do not want to change from one system to another EHRS; they think this change may threaten their job security" (GM2)

Finally, Analyst 1 revealed that some end-users still prefer to use a paper-based system instead of using the electronic-based system.

"Some users prefer to stay on the paper system." (Analyst 1)

\subsubsection{Connectivity issues}

Connectivity is another challenge to the implementation of an EHRS in PHCs. In this context, linking PHCs is almost impossible, due to lack of internet connection.

"The connection of PHCs with each other is difficult, it's nearly impossible" (GM 1)

"The main problem was the impossibility of connecting the EHRS in the PHCs with the internet." (Analyst 1)

Moreover, connectivity issues are directly associated with failure of the previous EHRS implementation project.

"Connectivity between the PHCs is considered a big challenge which led to failure of the project." (Analyst 1)

However, "A few PHCs have successfully been linked to the Internet." (HD2)

\subsubsection{The efficiency of the implemented EHRS}

When examining the previous EHRS, inefficiency was found to be another reason for implementation failure. In addition, one of head of departments described the previously implemented EHRS as "a modest system". Moreover, decreased efficiency of the implemented EHRS was considered to be due to a lack of comprehensiveness, where the required functionality to provide a proper healthcare service to the patients was not being integrated. Therefore, the previous system was lacking many of the features compatible with the functions of PHCs. 
"The implemented EHRS is a modest system and not one of high efficiency." (HD 3)

"They implemented EHRS, but they were not comprehensive and did not have all the required characteristics and functions, such as a CDSS. The coding in the system differs from the user's coding system in the Ministry, and this is one of the defects which curtailed the advantage of the current EHRS." (GM 1)

In addition, the implemented EHRS did not meet the expectations of the end users.

"As far as I am concerned, the previous EHRS does not meet users' expectations and it was not fully efficient." (DHD 1)

"I believe that the current EHRS has not come up to the expectations of the users." (HD 2)

However, the software developer argued that the implemented EHRS was efficient enough to meet the end-user's expectations.

"The EHRS meets the requirements of the PHCs more than you think; the proof is that there are still some PHCs using the EHRS. " (SD1)

\subsubsection{System usability}

Some participants stated that the end-users of the EHRS found the system easy to use. For instance, Analyst 2 stated, "All users agreed that the system is very easy to use" (Analyst 2), and GM1 stated, "The EHRS is honestly easy to use".

However, according to the software developer, some doctors expressed dismay at the number of screens, particularly during movement between commands, and time was wasted as a result.

"The flow of many screens has upset some doctors. They do not want to move through many screens and from one screen to another - that takes a lot of time." (SD1)

This section evaluates the implemented EHRS from a project team perspective. The findings show that the EHRS was implemented in only 150 PHCs out 2259. However, the implemented EHRS was terminated due to several causes. Lack of technical support and connectivity were the most common causes that led to the failure of the implemented EHRS. The project team evaluated the implemented EHRS on a regular basis through direct meetings with the EHRS end-users and through surveys distributed to the end- 
users. The outcomes of these evaluations show that the system was very useful and has had a positive impact on the EHRS end-users' daily work. In addition, the evaluation illustrated that the system was easy to use. However, the implemented EHRS faced several issues, such as lack of connectivity and technical support. Adequate training was provided to all EHRS end-users from the first day of the implementation process. 


\subsection{Summary}

This study explores, qualitatively, EHRS implementation in Saudi PHCs from a project team perspective. The majority of the data addresses the pre-implementation and postimplementation phases. Initially, the participant started to provide details about the other pre-implementation procedures such as project team selection and communication. Then participants went to describe in detail the plan to implement the EHRS in the PHCs. Hence, the analysis of semi-structure interviews shows that the plan to implement an EHRS, in the Saudi PHCs, is relatively in line with other plans described in the literature, despite the differences in the scale and the location of the project. In this context, the findings reveal that the majority of factors influencing EHRS implementation were taken into consideration, by the project, team during the pre-implementation phase. For instance, organisational level factors such as training, support, legal issues and organisational workflow and redesign, were a concern of the project team during the preimplementation phase. In addition, other factors related to technology and end-users were included in the EHRS implementation plan.

Thereafter, I asked the participants about the impact of CM and FR on EHRS implementation in PHCs in SA. The findings show that both CM and FR have a positive impact on EHRS implementation in PHCs in SA. In particular, factors such as interoperability, teamwork communication and software selection were positively influenced by CM. Factors such as preparation of infrastructure, provision of training and software selection were the most positively influenced by FR. Moreover, participants discussed several barriers to implement large-scale EHRS in the Saudi PHCs. The findings of this study revealed that barriers are greater than facilitators. In addition to barriers, participants provided several facilitators. For instance, financial resources are determined to be the most influential to EHRS implementation In regard to the barriers, technical factors such as connectivity and preparation of technical infrastructure formed the greatest barrier to implementation. Therefore, the Saudi MoH has taken several steps to overcome these barriers, such as co-operation with TCs in SA to perform infrastructure configuration tasks.

Several of the identified barriers had a direct impact on the previous project and led to project failure (e.g. changing people, lack of connectivity and lack of technical support). 
Therefore, went to asked about the EHRS previously implemented in PHCs in SA. The findings revealed that EHRS was implemented in 150 PHCs and was considered as a pilot to the previous project. However, the findings also revealed that the implemented EHRS was easy to use, and improved healthcare quality and end-user productivity.

To sum up, the Saudi MoH now attempts to implement new EHRS, due to the failure of the former project. Although several barriers found to have negative impact on the success of the EHRS implementation, the Saudi MoH has taken several steps to overcome these barriers. On the other hand, facilitators such as FR, CM, PHCs readiness and perceived usefulness of an EHRS contributed to the EHRS implementation in the PHCs in SA. The following chapter will discuss thesis limitations, strengths, weaknesses and recommendations for future work. In addition, the next chapter will discuss in detail the main findings of the four studies conducted in this thesis within the context of the existing literature. 


\section{Chapter Eight: Discussion}




\subsection{Introduction}

This thesis set out to explore the readiness of PHCs for the implementation of a largescale EHRS, determine the barriers and facilitators to the implementation of EHRS in PHCs in SA, and to find out the impact of FR and CM. To achieve these aims, four different methodologies (three quantitative studies and one qualitative study), three different populations, and four different tools (see Chapter Three) were used. This chapter will compare and contrast the findings from all four studies, to clearly address the main objectives of this thesis. Moreover, this chapter will provide a context for the findings of this thesis in relation to other published literature in the field.

\subsection{Study limitations}

A number of limitations need to be noted regarding the present study. One of the main limitations, that negatively impacted the data collection of this thesis, was a disclosure agreement between the Saudi MoH and IBM (consultant partner with the Saudi MoH). Initially, the decision was made to conduct five studies to achieve the main aim of this thesis. However, I was unable to conduct the fifth study, which aimed to "assess PHC readiness for EHRS implementation" through documentation analysis. Although I obtained all the required ethical approvals, the Saudi MoH refused to provide me the required documents due to their contract with IBM. According to this partnership, both parties agreed not disclose any information or documents concerning the E-health strategy. Documentation analysis can be useful tool to describe the implementation plan with consideration of good plan described in previous literature (see Chapter Two). As illustrated in Chapter Two, documentation analysis ranked as the third frequent data collection method used in the field of the EHRS implementation.

Despite the facilities provided by the Saudi $\mathrm{MoH}$, this thesis was impacted negatively by the type of project management utilised at the Saudi $\mathrm{MoH}$, which is purely centralised (see Chapter One). This is where all the decisions, relating to EHRS implementation projects, are taken centrally by a small group of people in two different departments of the Saudi MoH, namely the IT department and the PHC department. This type of management has led to a widening of the gap between the project team and the PHC staff. Therefore, PHC staff have less knowledge about certain aspects related to EHRS 
implementation in their workplace, such as resources, management structure and administration support.

During the data collection phase, several changes were made at the senior management and ministerial level in the Saudi MoH. To the best of my knowledge, during 2014 and 2015, six different ministers were appointed by the Saudi MoH. These personnel changes impacted negatively on the data collection process, where some study participants had been recently appointed in their new positions and were therefore less knowledgeable about the strategic implementation plan; others had no idea about the former project. Furthermore, several of the project team and policymakers who had been booked for an interview were laid off prior to the interview date, which affected the sample size of the semi-structured interviews.

I submitted a proposal two years before the commencement of my $\mathrm{PhD}$. The Saudi $\mathrm{MoH}$, at this time, was piloting the former system in some PHCs. Due to the changes at the senior manager and ministerial level, mentioned earlier, the new staff decided not to pursue the previous project and commenced with a plan to implement a new EHRS. They argued that the previous project had serious issues, which hindered the success of the EHRS implementation. Consequently, I found myself needing to examine two different projects to be able to fully explore the implementation of large-scale EHRS in Saudi PHCs. This subsequently led to some repetition (see Chapter Seven).

Based on the regulations of the Saudi Cultural Bureau in London (my sponsor), $\mathrm{PhD}$ students are allowed to travel to SA to collect their data for up to three months, which negatively impacted the data collection process. Due to these regulations, I decided to conduct the data collection via an electronic method, so a questionnaire was compiled for both studies conducted in the PHCs. Time issues also negatively influenced the data collection process, where the sequence of studies was based on the accessible population. Therefore, one of the main weakness of this thesis was the sequence in which the studies were conducted. Although the semi-structured interviews were conducted before two of the questionnaire-based studies, it was felt that a sequential explanatory mixed-method of conducting the semi-structured interviews after all questionnaire-based studies may have provided a better understanding of the implemented EHRS. This can improve the coherence of the thesis and help gain a better understanding of the findings. In addition, 
since the qualitative semi-structured interviews were conducted with the project team, a sequential explanatory mixed-methods will be beneficial to gain further discussion and interoperation of quantitative findings, especially those requirements and disagreements reported by PHC staff and EHRS end-users of former EHRS.

Interviews were carried out with participants whose first language was Arabic, for purposes of convenience and to avoid any misunderstanding. However, the majority of the data analysis time was spent on the transcription and translation of the interviews from Arabic to English. Some of the interviews were two hours long, and more than a week was spent transcribing each interview. Due to the amount of obtained data, the total interview, a substantial amount (five months) of my $\mathrm{PhD}$ candidature time was spent carrying out translation, transcription and validation.

The EHRS was implemented across PHCs located in different regions of SA, and, it was very difficult to travel to all these PHCs to distribute the questionnaires of studies two and three. Due to the time limitation issue mentioned previously, as well as travel difficulties, I decided to utilise an electronic-based survey. However, the official e-mail was not activated, so the sample was reduced from all PHCs that have an EHRS to a selection of PHCs based on their geographical location (see Chapter Three). 


\subsection{Readiness of SA PHCs to implement an EHRS}

As illustrated in Chapter Two, readiness assessments need to be conducted in the early stages of the pre-implementation phase. Therefore, an initial objective of this thesis was to assess PHCs readiness for the implementation of the EHRS in SA, since the Saudi $\mathrm{MoH}$ is planning to implement the EHRS in all PHCs and according to my best knowledge the Saudi MoH still in early phases and no EHRS implemented (see Chapter One). Due the importance of readiness assessments to the implementation of the EHRS, I, therefore, conducted three studies using both quantitative and qualitative approaches. This triangulation of methods aimed to enhance the validity of the findings. Data were obtained from two different perspectives: the project team, and PHC staff (see Chapter Three). In reviewing the literature, no data about readiness of Saudi PHCs for implementation of a large-scale EHRS was found.

Although the majority of the findings related to readiness were obtained via a pre-existing questionnaire (see Chapter Three), this questionnaire has never been used to measure the level of readiness of PHCs for EHRS implementation. In addition, there has been limited research conducted to measure the readiness of healthcare organisations in developing countries (Alasmary et al., 2014), which distinguish the findings of this thesis. Saleh et al. (2016) conducted a study in Lebanon and identified that no previous studies had been conducted to assess PHC readiness to implement a new EHRS in Arab countries. Therefore, the research in this thesis is one of only a few that have measured the level of readiness of PHCs for EHRS implementation in developing countries. It is also the first to be carried out on the large-scale implementation of an EHRS in PHCs. It is also of note that the readiness assessments in this thesis were conducted in 2014, before the research by Saleh et al. (2016), which means that the readiness assessments in this thesis are the first to be carried out in an Arab country.

Findings, from studies one, two and four to assess PHCs readiness for the implementation of a large-scale EHRS, illustrated different level of readiness, due to the disparity in responses between project team and PHCs staff (will be discussed in the following sections). Moreover, findings from Study Four revealed disparity in responses between project team themselves. For instance, while the Deputy Head of Department stated that 
the PHCs are ready for the new EHRS, the Software Developer argued that some the PHCs still not ready yet.

It is worth noting that the majority of the participants in Study One were males, which may bias the data and limit the generalisability of the findings. However, there was no significant differences between males and females. The ratio of respondents however reflects the proportion of females to males at the headquarters of the Saudi $\mathrm{MoH}$ (see Chapter Four). Therefore, recorded feelings towards EHRS implementation are mainly based on male perceptions. The data in Study One were not normally distributed due to the fact that the responses to most questions were positive. This could reflect a true agreement by the respondents to the statements presented or could be due to a number of other factors. The data collection instrument utilised in Study One was one that had been previously developed and used in other studies (see Chapter Three). Using an existing instrument may have limited the responses for participants, with them being forced to select one of the categories available, with the positive categories perhaps being the best available to them.

It could also be that individuals working in the in the Saudi MoH may be selecting the most socially desirable responses and being overly positive regarding EHRS implementation in the PHCs, especially after the previously failed project. Respondents could also have been mindful of the new stricter changes at governmental level and there may have been apprehension to respond negatively for fear of reprisal. I, however, reassured participants prior to their participation regarding the fact that the data would be confidential and only used for research purposes only. To get better understanding of the nature of the participants and their ability to provide these facts. Question regarding their involvement in the project has been added. The purposes of adding this question was to determine the level of knowledge between those who are directly involve and the others who are not. However, differences test shows no significant difference between who are directly or indirectly involved.

Adding a "don't know" response may have provided more accurate responses especially with factual questions rather than attitude questions. Therefore, if I were to conduct this research again using the same instruments, I would add a "don't know" option. Although readiness assessments were negatively impacted by $\mathrm{CM}$ (see Section 8.2), conducting 
both readiness studies simultaneously in future and using one data collection instrument may enhance response rates and save time. The data collection instruments for both Study One and Study Two could be combined and then modified by providing a "don't know" response option.

\subsubsection{PHC readiness for EHRS implementation at the individual level}

Results, from studies one, two and four to assess readiness, illustrated a high level of readiness at the individual level. Both project team and PHC staff expressed high levels of readiness to the implementation of the new large-scale EHRS. Furthermore, PHC staff expressed a very high level of awareness, acceptance and willingness towards the implementation of the EHRS in their workplace. On the other hand, findings from Study Two and those from Saleh et al. (2016), revealed that gender and age differences had no association with individual readiness for the implementation of EHRS. In contrast, previous research conducted in three hospitals in Ethiopia documented that males displayed a higher level of readiness toward the implementation of EHRS than females (Biruk et al., 2014). In addition, Biruk et al. (2014) documented that younger healthcare professionals showed a greater readiness for EHRS implementation.

My findings also showed that experience in the workplace made no significant difference to the level of individual readiness for EHRS implementation (see Chapter Five). Likewise, Saleh et al. (2016) found that experience at the workplace had no significant association with individual readiness. When examining the association with occupation, my study findings showed no difference in relation to different occupations. For example, both physicians and nurses express the same level of readiness toward the implementation of the EHRS. Contrariwise, Saleh et al. (2016) detected a significant association between the level of readiness of PHCs staff and their occupation, with nurses displaying a higher level of readiness compared with physicians. It is worth noting that my research included a wide range of PHC staff from various occupations, including lab-technicians and dentists, which have not previously been surveyed in other research studies especially in developing countries.

The findings of Study Two also illustrated that years of experience in using computers had no influence on the level of individual readiness for implementation. These findings 
contrast those of Biruk et al. (2014); Kuo et al. (2013); Saleh et al. (2016); and Terry et al. (2008), who documented that users with more computer experience had a higher level of individual readiness. It is of note that the majority of the PHCs ( $n=300,86.9 \%)$ have more than five years' experience in using PCs (see Chapter Five), indicating a high level of readiness if we consider that computer skills are a predictor of the level of readiness (see Chapter Three). One of the interesting findings obtained from the semi-structured interviews was that experience with different types of technology other than PCs, such as mobile phones, was found to have a positive impact and encourage individuals to use the EHRS and increase the level readiness to implement new technology (see Chapter Seven).

The findings from Study Two illustrated that PHC staff expressed a high level of enthusiasm to be involved in EHRS implementation in their workplace. However, the participants' response to "Staff are typically involved in EHRS implementation" was mostly 'disagree', at $67.2 \%$, and their response to "Staff have been included in decisionmaking processes" was also mostly disagree. This indicates that PHC staff are not happy with the current involvement mechanisms, which may affect their readiness (will be discussed in detail later in this chapter). In contrast, the findings from Studies One and Four illustrated that the involvement of PHC staff in the implementation process and planning was rated highly.

Despite the PHC staff's disagreement about involvement mechanisms in the EHRS implementation, $96.6 \%$ of them endorse EHRS implementation in their workplace. Furthermore, they were asked whether they would resist EHRS implementation or not, only $9.1 \%$ said they would which reflect their willingness to the EHRS implementation. Biruk et al. (2014) found that those who have good knowledge about the EHRS and are also willing to use the EHRS are more likely to be ready for its implementation. It is of note that the awareness scale in Study Two has no "don't know" response option, which may have influenced the participant response. Providing a "don't know" answer may allow the participants to express their awareness about the benefits of the EHRS more accurately. Although level of education was found to be a predictor variable of the level of individual readiness for EHRS implementation (Saleh et al., 2016; Yusif et al., 2017), Study Two failed to examine this factor. Hence, future researcher may need to consider this predictor variable when readiness assessment is being carried out. 
At the project team level, involvement in previous EHRS implementation projects has been considered as a key predictor to the level of project team readiness to the EHRS implementation (see Chapter Three). The findings show that the project team at the Saudi $\mathrm{MoH}$ had a good level of readiness due to their knowledge about previous EHRS implementation, with more than half of the project team (58\%) having been involved in similar projects previously. In addition, the project team members agreed that "there is $a$ lot of knowledge about the on-going development needs of EHRS support staff". However, it is worth noting that the project at the Saudi MoH relies on capitalising on the experiences of other organisations and other countries in the development of strategic implementation plans and policies (see Chapter Seven).

\subsubsection{PHC readiness for EHRS implementation at the training and technical support levell}

The results of studies one, two and four illustrated a moderate level of readiness in terms of training and technical support. It was indicated that awareness campaigns are essential and can be included as part of training courses (see Chapter Seven). Similar to my findings, Steininger et al. (2014), and Allen et al. (2000) also found that providing adequate awareness campaigns would contribute to enhancing end-user readiness for the introduction of a new EHRS and reduce their resistance. Therefore, it is highly recommended to provide awareness campaigns for large-scale projects that may face direct contact challenges with stakeholders. According to the Saudi MoH plan, training will be provided to EHRS end-users before implementing a system in order to raise their competence level and avoid any resistance or training issues (see Chapter Seven).

Both the project team, in Study One, and PHC staff, in Study Two, were asked about the availability of adequate training and delivery methods. Their responses were contradictory. While the project team's responses showed a high level of agreement with regards to whether training had been provided to PHC staff, with a total agreement of $77.4 \%$, PHC staff disagreed, with a total disagreement of $63.7 \%$. This result indicates that the more than half of PHC staff were yet to receive training. In addition, responses to open-ended questions highlighted the need to provide adequate training (see Chapter Five). Although the project team stated that training courses will be held in the preimplementation phase, no EHRS has been selected yet, which could be the reason for a 
lack of training at this stage. It is possible, therefore, that training sessions will be established once the EHRS has been selected. The disagreement of PHC staff indicates that PHCs remain at a lower level of readiness in this regard. However, $83.3 \%$ of the project team agreed that "the project budget includes training/retraining costs".

Moreover, the planning has taken into consideration several methods to provide adequate training and support. My findings and those of Keshavjee et al. (2006); Piliouras et al. (2011), determined that relying on vendors to provide training and support to end-users is a useful way to overcome any challenges related to the provision of training and support, particularly in large-scale projects (see Chapter Seven). In addition, the approaches planned by the $\mathrm{MoH}$ involve training a large number of staff for a large-scale implementation project so these trainers can then train the end-users, this concept known as 'train the trainers'. These findings are consistent with data obtained from previous studies (Slight et al., 2014) which suggest applying the concept of 'train the trainers'. It is of note that the study by Slight et al. (2014) was conducted in secondary care in the UK. Other possible training methods have been agreed, such as distributing guidance leaflets to help end-users and educate them.

\subsubsection{PHC readiness for EHRS implementation at the processes level}

\section{Project team communication}

The project team responses illustrated the prominent and effective role of the IT department in facilitating EHRS implementation. The findings show the project team's keenness to have channels of continuous and effective communication among all stakeholders at all levels, which have also been postulated by previous studies (Carayon et al., 2014; Lorenzi et al., 2009; Unger et al., 2014). The findings also emphasised the importance of the presence of representatives from the project team in meetings or committees. The presence of key members of the project team in all communication methods, as mentioned in Chapter Two, can enhance the level of readiness for EHRS implementation. As identified in Chapter Seven, project team communications were made through three different methods: meetings, committees, and workshops. Regular meetings and workshops were also found to be useful methods of ensuring proper communication 
among project team members in previous studies conducted in developed countries (Pitcher, 2010; Tsiknakis \& Kouroubali, 2009).

\section{Conduct consultations}

As part of the project team preparation for the implementation of this large-scale project, they conducted several consultations to enhance the readiness of the PHCs, as well as to formulate a well-defined plan. As revealed in Study Four, consultations were found to have a prominent role in enhancing the success of the implementation of this large-scale project, as well as to overcome any barriers that may have hindered its success. One of the most interesting findings to emerge from the semi-structured interviews (see Chapter Seven) is that such a large-scale project should consider a number of consultations and attempt to benefit from the experiences of other countries or experts with extensive experience in the field of EHRS implementation. This is due to the magnitude of the project and the need to take extreme care to avoid mistakes that can be extremely costly. Reliance on consulting services and taking advantage of the experience of others are positive measures that will contribute to the success of any implementation project. The findings from this thesis suggest that conducting a multi-stage consultation during the planning phase could avoid mistakes or drawbacks that may lead to project failure. These are country level, organisation level and individual level (see Chapter Seven). Conducting consultations can assist to overcome shortage in HI experts (will be discussed further later in this chapter)

Conducting consultations was found to be predictor of the level of readiness of the healthcare organisations to the introduction of large-scale EHRS, and only emerged following the analysis of Study Four. It would have been beneficial if the data collection instrument used in Study One considered this important factor. Therefore, future researchers who are interesting in assessing healthcare organisations readiness to implementing EHRS especially large-scale projects, may need to consider adding few items to the utilised data collection instrument in Study One about conducting consultations. 


\section{Workflow and business structure redesign}

The findings from Study Four identify that the business structure of PHCs has been redesigned to comply with EHRS implementation (see Chapter Seven), which indicates that PHCs are ready for the EHRS at the process level. In contrast, healthcare organisations in the UK have recorded lower readiness due to the variation between workflow processes and new IT systems (Lennon et al., 2017).

\subsubsection{PHCs readiness for EHRS implementation at the management structure and administrative and financial support level}

The results from studies one and four illustrated that PHCs are at a high level of readiness at the management structure and administrative and financial support level. However, due to a lack of awareness of the PHC staff in regard to certain administrative aspects related to EHRS implementation in Saudi PHCs, findings about management structure were mostly obtained from the project team. The quantitative and qualitative studies assessed the strategic plan and its compatibility with the implementation of the EHRS project. For instance, project team responses show a very high level of agreement (74.2\%) with the statement, "The IT strategic plan is an effective guide for the organisation's EHRS implementation processes". This illustrates a high level of readiness at the management structure level. A well-designed strategic plan can directly link with consultations made during the planning phase, especially for large-scale projects. In contrast, the readiness of healthcare organisations in the USA were found to be low at the planning level (Cherry, 2011)

Another interesting finding to emerge from analysis of studies one and four was that the readiness of PHCs at the resource level was high. The findings show that the Saudi MoH pays great attention to the project in general by providing an adequate budget. About ninety three percent of the project team agreed that "funding is adequate for completion of EHRS implementation". Thus, this result demonstrated the great impact of the financial aspect on the success of EHRS implementation. In addition, the finding shows that "the project budget is consistent with the organisation's strategic plan'. In contrast, previous research conducted in the UK revealed that the level of readiness with respect to FR was 
found to be low and considered to be a constraint to EHRS implementation (Lennon et al., 2017).

\subsubsection{PHC readiness at the technological level}

Although Study One was designed to assess PHCs readiness for EHRS implementation, it failed to examine the technical infrastructure as one of the readiness assessments criteria (see Chapter Three). Technical infrastructure was identified in studies two and four, and again it would be useful in future if technical infrastructure was included in the data collection instrument used in Study One. Findings, from studies two and four, illustrated that PHCs are at a lower level of readiness with respect to technology than they are in relation to other factors, such as resources. This can be inferred from the numerous requests $(n=25)$ to improve the PHCs infrastructure (see Chapter Five). These requests indicate inadequate infrastructure, particularly when they raised claims to provide an "Internet connection" in their workplace. Infrastructure aspects, such as connectivity, were recorded to be inadequate and not meeting the requirements for EHRS implementation. These issues represent a challenge for the Saudi MoH.

The findings also reveal that the available hardware is not compatible with the new EHRS. Many of the participants in Study Two requested up to date hardware that is compatible with the new project, such as desktops and printers. These findings are in line with Lennon et al. (2017); and Sahay (2001) who also argued that developing countries are still behind developed countries in terms of technical infrastructure. However, Cherry (2011) reported a low level of readiness in developed countries, such as USA, with respect to the provision of appropriate hardware. Lennon et al. (2017) also found that the level of readiness in both hospitals and PHCs in the UK was low with respect to technical infrastructure. Furthermore, Biruk et al. (2014) documented low infrastructure readiness in Ethiopian hospitals. Contrariwise, Saleh et al. (2016) found that PHCs in Lebanon were at a higher level of readiness in terms of hardware. Most importantly, large-scale projects can suffer dramatically due to poor infrastructure, systems interoperability and other technological challenges. If these challenges are insurmountable and improperly handled, they may lead to the failure of EHRS implementation projects. All of these findings show that, poor technical infrastructure was the most significant factor affecting readiness for EHRS 
implementation in healthcare organisations in developing and developed countries alike, especially when implementing large-scale projects.

\subsection{Facilitators of EHRS implementation in PHCs in SA}

In addition to readiness assessments, the present thesis was also designed to determine the facilitators of and barriers to EHRS implementation in PHCs in SA. Similar to the previous section, the identification of the facilitators and barriers were determined through three different studies (two quantitative and one qualitative), with responses gained from a diverse population (project team, PHC staff and EHRS end-users) to improve the validity of the findings through triangulation. Results, from studies one, three and four, showed that the main facilitators to implementing a large-scale EHRS in the Saudi PHCs were FR, leadership and management, specifications of the PHCs, perceived usefulness, and EHRS usability. It is worth noting that findings from Study Four may be impacted by the current use of the EHRS, since the former EHRS failed, about $76 \%$ of EHRS end-users are no longer using the system in their workplace, some of them even didn't use the EHRS for more than five years. Thus, this may negatively impact their responses. In reviewing the literature, very little was found regarding the impact of CM and FR on the implementation of EHRS, especially large-scale projects (see Chapter Two).

\subsubsection{Financial resources (FR)}

Results from studies one and four to determine the impact of FR, illustrated that FR had a very high positive impact on facilitating the implementation of large-scale EHRS in the PHCs and contributing to overcome many challenges. This could reflect a true agreement by the respondents to the statements presented in Study One or could be due to a number of other factors. Using Likert scale may have limited the responses for participants (see Chapter Four), with them being forced to select one of the categories available, with the positive categories perhaps being the best available to them. However, the participants provided negative and no opinion response. Therefore, if I were to conduct this study all over again, I will rephrase the questions to be negative or neutral. However, findings from 
Study One were then further supported by the findings of the semi-structure interviews (see Chapter Seven).

The findings showed that the Saudi MoH did not face any financial constraints during the implementation of the EHRS projects. Thus, the influence of this factor has been examined against some of the main factors found to have a direct relationship with FR (see Chapter Three). This thesis is the first comprehensive investigation of the impact of the FR on EHRS implementation in SA, whereas the impact of FR has been examined against a wide range of factors which have been presented in previous literature (see Chapter Two). Consequently, the factor most influenced by FR was software selection, where $93.5 \%$ of project team agreed that FR assists in the selection of high-quality software. It was perceived that FR could have a beneficial effect on software selection, allowing more flexibility to select the best vendors to implement EHRS in PHCs and then enhance the system interoperability. Although, preparing adequate infrastructure is very costly (Ross et al., 2010), it was another factor that significantly influenced by the provision of the FR in a positive way (see Chapters One and Four). Another interesting finding (from studies One and Four) was that FR facilitated the provision of training and technical support, which had previously been reported as a barrier to implementing a large-scale EHRS. Ninety percent of the participants, in Study One, agreed that FR has a very positive impact on the provision of training. In addition, in a semi-structured interview, Analyst 2 said, "FR is essential and has a very positive impact, particularly on training".

Although findings from studies One and Four illustrated that FR was one of the main facilitators to the implementation of the EHRS in Saudi PHCs, others found that the cost of implementation was one of the main barriers, and the Saudi healthcare organisations struggle to support their project due to FR shortages (Altuwaijri, 2008; Hasanain et al., 2014; Khudair, 2008). Likewise, internationally, the cost of EHRS implementation is classified as a barrier to the success of the projects (e.g. Gagnon, Nsangou, et al., 2014; Jha, Bates, et al., 2009; Jotkowitz et al., 2006; Koivunen et al., 2008; Kruse, Kristof, et al., 2016; Leon et al., 2007; Ludwick \& Doucette, 2009b; McAlearney et al., 2005; Nakamura et al., 2010; Walji et al., 2009). It is worth noting that my study is the only one which has examined the impact of the FR to on the implementation of large-scale EHRS in the Saudi PHCs. 


\subsubsection{Leadership and management}

Overall, the findings from studies One and Four identified that strong leadership and appropriate project management play a key role in the success of large-scale projects. Effective leadership, at the senior management level, can contribute to the success of the implementation of a large-scale project, fifty percent of the project success can rely on strong and effective leadership and management (see Chapter Seven). However, leadership and management issues have been documented, in previous literature, as one of the barriers to EHRS implementation in SA (Khudair, 2008). In some cases, these issues may lead to the failure of EHRS projects (Boonstra \& Broekhuis, 2010; Khudair, 2008).

This thesis is the first comprehensive investigation about the impact of the $\mathrm{CM}$ on largescale EHRS implementation in PHCs. Hence, results, from studies One and Four to evaluate the impact of $\mathrm{CM}$, showed that this barrier may not exist, when implementing a large-scale EHRS in PHCs that utilise CM and illustrated that CM had a positive impact on EHRS implementation. Similar to the previous section, this could reflect a true agreement by the respondents to the statements presented in Study One or could be due to a number of other factors (see Section 8.4.1). However, these findings were further supported by the findings of the semi-structure interviews (see Chapter Seven). Moreover, these findings are in line with those by Safdari et al. (2015), who also stated that $\mathrm{CM}$ can contribute to the success of the EHRS implementation. It is worth noting that research by Safdari et al. (2015) carried out in secondary care organisations. My findings and those by Safdari et al. (2015) are in disagreement with the findings by Ekvall (1996), who argued that CM has some disadvantages such as delaying projects, resistance to change, and a decrease in the level of innovation within the organisation. This conflict raises doubts about the impact of the $\mathrm{CM}$, which require further investigation in the future.

The impact of CM was examined with consideration of several factors that influence the EHRS implementation (see Chapters Four and Seven). Consequently, systems interoperability tops the list of factors influenced positively by CM. Therefore, CM can be a lifeline for many organisations and project teams that have encountered interoperability issues during EHRS implementation. On the basis of the findings of this thesis, it appears that $\mathrm{CM}$ helps facilitate and overcome several difficulties faced during 
software selection. Therefore, the factors that are affected positively by CM are decisionmaking, planning, scale of the project and teamwork communication. The study identified that unilateral decisions and plans that are supported by everyone have a positive impact on huge projects, and that this can only be achieved through CM.

Although CM has been found to be beneficial for large-scale projects, it was highly recommended to move toward semi-centralised management (see Chapter Seven). This can enhance other stakeholder awareness, particularly EHRS end-users and, consequently, semi-centralised management can improve the level of satisfaction of those end-users. To make better decision regarding which type of management is better to implement large-scale EHRS, further research is needed, in the future, to examine the difference between the centralised and semi-centralised management. CM lead to unification of workflow and business structure of all PHC centres in SA. The following section will discuss this factor in detail.

\subsubsection{PHC specifications}

One of the results of this thesis, which has not been addressed before, is that the nature of the PHC workflow and business structure which is common to all PHCs in SA is a facilitator of EHRS implementation. This harmonisation is due to the CM system adopted by the Saudi $\mathrm{MoH}$ (see Chapter One). In this context, this harmonisation facilitates software selection, where one system can be implemented in all PHCs in SA. In addition to software selection, training courses also can be unified due to the similarities in PHC healthcare function, workflow and business structure.

Furthermore, the findings illustrate that the size of the PHCs in SA is another facilitator of EHRS implementation. As illustrated in Chapter Two, the size of the healthcare organisation has been recognised as an influential factor (Ash \& Bates, 2005; Kruse, Kothman, et al., 2016). In this regard, the results of studies One and Four revealed that the project team benefited from the small size of the PHCs. In contrast, the findings of previous research show that larger healthcare organisations such as hospitals are more flexible and have a higher level of readiness than PHCs or other small healthcare 
organisations (Jones \& Furukawa, 2014). Others argued that EHRS adoption is lower in small practices compared to large practices (Ancker et al., 2013; DesRoches et al., 2012).

\subsubsection{EHRS usabillity}

Results, from studies Three and Four, illustrated that EHRS usability was one of the facilitators to implementing a large-scale EHRS. These findings are in agreement with those by Ludwick and Doucette (2009b), who documented a positive relationship between the usability of the EHRS and the adoption rate. Others argued that usability issues can act as constraints to the implementation of IT in healthcare organisations (Gagnon, Nsangou, et al., 2014; Khajouei et al., 2011; Kruse, Kristof, et al., 2016; Middleton et al., 2013; Yusof et al., 2008). Moreover, as illustrated in Chapter Two, system usability is directly associated with end-user satisfaction (Gagnon et al., 2016; Khajouei et al., 2011). Accordingly, EHRS end-users recorded very high levels of satisfaction with system usability. Compared with previous literature, these findings are in contrast with those presented by Cresswell et al. (2012), which recorded dissatisfaction with system usability.

Feedback statements reflect the importance of involving EHRS end-users in implementation. In addition, the EHRS end-users identified a few usability issues that may be of interest to the project team (see Chapter Six). Therefore, it is highly recommended that EHRS end-users are involved and that consideration is given to their feedback and recommendations, either during software selection or when system enhancement is taking place to improve the system usability. While numerous studies have documented adverse effects on the quality of care, medication errors, EHRS enduser errors, and patient safety resulting from usability issues (Khajouei et al., 2011; Middleton et al., 2013; Zahabi et al., 2015). This thesis wasn't able to evaluate the relationship between usability issues and consequent factors, such as medication errors. Therefore, further researches is needed to determine this relationship in PHCs settings. 


\subsubsection{Perceived usefulness of the EHRS and system efficiency}

While the findings, from studies Three and Four, illustrated that perceived usefulness are other facilitators to the EHRS implementation, previous literature revealed that the EHRS was not useful and was considered to be a barrier to the EHRS implementation (Boonstra et al., 2014; Clarke et al., 2013; Nguyen et al., 2014). Benefits such as data accessibility, time saving, cost reduction and improved productivity were the things that end-users like about the EHRS implemented in PHCs in SA. The findings also show that EHRS endusers gave positive feedback about data accessibility, accuracy, improved productivity and time saving as a result of the system. Although these findings differ from those of several published studies (Chen et al., 2009; Handel \& Hackman, 2010; Huerta et al., 2013; Miller et al., 2005; Redd et al., 2014; Zandieh et al., 2008), which argue that EHRS decreases staff productivity, they are consistent with those of Cheriff et al. (2010); and Lorenzi et al. (2009).

The findings of studies Three and Four are consistent with those of Gagnon et al. (2012); Gagnon, Nsangou, et al. (2014); Jha, DesRoches, et al. (2009); and Kruse, Kothman, et al. (2016), who also found that cost reduction constitutes a major facilitator of EHRS implementation. In addition, the findings illustrated that a higher perceived usefulness of an EHRS increases the end-user's willingness to use the system, which has been recorded as another facilitator to EHRS implementation. In addition to the above benefits, results (from studies Three and Four) illustrated that the EHRS contributed positively to patient outcomes. To sum up, while data accessibility, time reduction, and improved productivity are the aspects EHRS end-users like most about the system, the project team found cost reduction to be the most beneficial aspect.

While, studies Three and Four show an agreement between both project team and enduser perspectives on perceived usefulness, in facilitating EHRS implementation, EHRS efficiency was another factor of dispute between the project team and EHRS end-users. Although EHRS end-users argued that the EHRS was efficient, the project team argued that the system was inefficient and modest. The above findings raised queries about the feasibility of implementing another EHRS, while the previous system already satisfied the end-users in terms of perceived usefulness and efficiency. It also proved that perceived usefulness was not a cause of the failure of the previous project and should be 
taken into consideration when the decision is made to select a new EHRS. When examining the correlation between perceived usefulness of the EHRS with training and support, the findings show no significant correlation. These findings are in disagreement with those of Carr et al. (2010), who documented a relationship between perceived usefulness of an EHRS with training and support.

To sum up, this section provides several recommendations, which can facilitate the implementation of a large-scale EHRS. Firstly, policymakers need to consider providing sufficient budget for smooth implementation, particularly when decisions are being made regarding software selection. Secondly, centralised or semi-centralised management were found to be more effective in implementing a large-scale EHRS to unify decisions, policies and procedures. Finally, at a technological level, selecting a beneficial, efficient and easy to use EHRS can enhance EHRS end-user acceptance, which may then facilitate the success of EHRS implementation projects.

\subsection{Barriers to EHRS implementation in PHCs in SA}

As mentioned in Chapter Seven, an EHRS has been implemented in only 150 PHCs out of 2259, and due to the barriers and challenges, this project failed. Therefore, it is worth investigating the causes behind this failure. Barriers determination was based on the Saudi $\mathrm{MoH}$ experience on previous and future projects. Findings about the barriers to the EHRS implementation were collected via questionnaire-based research and semi-structured interviews from two different populations: the project team, and EHRS end-users. Although several facilitators were found to have a positive impact on implementing a large-scale EHRS, several barriers to project success were identified. The most obvious finding, to emerge from the analysis of Study Four, is that there were many more barriers to the implementation of a large-scale EHRS, than there were facilitators. Consequently, results (from studies Two, Three and Four) illustrated that some barriers are unique to this particular project, such as the large-scale of the project. Other revealed barriers were changing individuals, lack of training, lack of technical support, lack of interoperability, geographical challenges, software selection, and lack of user involvement. 


\subsubsection{Scalle of the project}

This thesis is one of only a few research studies that have identified implementing a largescale EHRS as a major barrier, even though the PHCs specifications such as the size was document to be a facilitator. Moreover, although $\mathrm{CM}$ was shown to have a positive impact on EHRS implementation, barriers related to the scale of the project have raised doubts about the effectiveness of CM. As illustrated in Chapter One, the number of PHCs in SA will rise to 3600 centres, which may increase the complexity of the implementation of the EHRS.

To overcome this barrier, it has been suggested that such large-scale projects need to be broken down into smaller projects. For instance, the EHRS should be implemented in different regions (zones) and different timeframes to facilitate the management of the project. Moreover, there should be agreements with more than one vendor to reduce the risk of failure due to, for example, the inability of the vendor to implement a large-scale EHRS project while maintaining certain criteria, such as system interoperability.

\subsubsection{Shortage of experts and changing individuals}

Although the findings, from Study Four, illustrated that FR can assist to overcome this issue, it still considered to be a barrier to the implementation of large-scale EHRS in the PHCs, especially in developing countries. These findings are in line with those of Lennon et al. (2017); and Ludwick and Doucette (2009b), who also revealed that developed countries, such as the UK, encountered a shortage of experts during EHRS implementation. Hence, a shortage of experts is considered to be a barrier for both developed and developing countries. However, findings from Study Four, strongly recommended hiring experts in HI and IT from other organisations or other countries, especially when the decision is being made to implement large-scale EHRS. The availability of experts in HI and IT can be considered as a predictor of the healthcare organisations readiness to the introduction of new EHRS. However, the data collection instrument in Study One failed to include items to examine the availability of experts to promote the success of the EHRS implementation, especially large-scale projects. Therefore, the future researcher may want to consider adding some items to assess the healthcare organisation readiness at the availability of HI experts' level. 
Another interesting finding was the barrier caused by the changing of executives and individuals in the project team, in particular key individuals. This obstacle may pose a greater threat to large-scale projects that have adopted CM, where CM can be defined as uniqueness of a small group in making crucial decisions. However, changing executives or other policy makers has also been identified as a barrier in small projects (Pare et al., 2008). Although CM was found to have a very positive impact on EHRS implementation (see Section 8.4.2), it may have negative implications. Changes at the executive and leadership level are often followed by changes to previously adopted policies and strategies, which can lead to project delay or even failure (see Chapter Seven).

\subsubsection{Lack of training and support}

Results, from studies Three and Four to determine the barriers to implementing largescale EHRS, illustrated that both technical support and training were found to be obstacles. However, some participants agreed that all EHRS end-users in PHCs have received adequate training since the first day of implementation. Moreover, the findings of studies Three and Four recorded a negative relationship between the large scale of the project and the provision of training and technical support. Although training and technical support have been recorded as barriers in small projects (e.g. Cherry, 2011; El Mahalli, 2015), they can be more complex in large-scale projects (see chapters six and seven).

The findings demonstrate a convergence of perspectives between end-users and the project team, in terms of classifying training as a barrier to EHRS implementation. Both EHRS end-users and the project team agreed that lack of training is a barrier to EHRS implementation. For instance, HD 1 said, "The lack of training is also among the problems", and Analyst 1 said, "Of the main obstacles is the training”. In addition, lack of training was classified as a major barrier by $83.8 \%$ of EHRS end-users, while $75.5 \%$ classified lack of technical support as a major barrier. Moreover, issues with training can be detected from the claims raised by the end-users in answers to the open-ended question (see Chapter Six). Demands to provide adequate training appeared twenty-six times, which constitutes twenty percent of all responses to that question. 
When examining the previously implemented EHRS in Saudi PHCs, provision of training was another factor of debate between the project team and EHRS end-users. While EHRS end-users argued that the training that they received was inadequate, the project team stated that "there was a very adequate training course" (HD 3), and also claimed that training sessions were provided to EHRS end-users from the first day of implementation and continuously.

Research conducted in PHCs in USA revealed that less than half of the staff perceived their training as adequate (Singh et al., 2013). In SA, the level of satisfaction toward training provided to EHRS end users is variable. For instance, Alasmary et al. (2014) examined the impact of training on EHRS end-user satisfaction in secondary care in SA and found that the end-users were satisfied with the training they received. However, other studies, conducted in secondary care in SA, revealed that EHRS end-users expressed dissatisfaction with the training and technical support (Alkraiji et al., 2013; Khudair, 2008).

The most obvious finding to emerge from the analysis in studies Three and Four is the direct link between the failure of the implemented EHRS in the Saudi PHCs and a lack of technical support. Moreover, the findings in (Chapters Six and Seven) show that project team perspectives are in agreement with EHRS end-user responses. They both agreed that technical support was inadequate.

Furthermore, results from studies Three and Four illustrated that training and technical support issues may negatively influence EHRS end-user acceptance. The findings show a low level of satisfaction toward the provision of training and support. This indicates that poor or inadequate training and technical support influence EHRS end-user acceptance and negatively impact their satisfaction. These findings match those observed in earlier studies conducted in SA (e.g. Alkraiji et al., 2013; Khudair, 2008). However, these two studies were conducted in secondary care. In contrast, previous research has found that technical support was one of the facilitators of EHRS implementation (Jha, DesRoches, et al., 2009; Wolf et al., 2012). However, Heyworth et al. (2012) argued that there was no correlation between the provision of technical support and end-user satisfaction. 
One of the interesting findings identified in this thesis is that, due to the importance of providing adequate technical support, between 18-20\% of EHRS implementation costs are dispersed on the provision of technical support. In addition, findings from studies One and Four illustrated that technical support should be available to the users twenty-four hours a day, which may require overtime expense. The study findings then strongly recommend allocating sufficient budget to the provision of training and technical support as an ongoing expense.

\subsubsection{Geographical challenges and infrastructure issues}

The geographical nature of the SA and its size is a major challenge facing the $\mathrm{MoH}$, although FR have contributed positively to overcoming this issue (see Chapter Seven). It is obvious that technical obstacles are more complicated than organisational barriers and human obstacles. The results, from studies Three and Four, revealed that geographical challenges represent the greatest barriers to the implementation of an EHRS in PHCs in SA. These findings are in line with a similar study conducted in the USA by Houser and Johnson (2008), who also found geographical challenges to be one of the main barriers to the EHRS implementation.

A negative correlation was seen between geographical challenges, poor infrastructure and lack of connectivity. This negative correlation was found to be one of the main reasons for the failure of the previously implemented EHRS (see Chapter Seven). This is due to the differences in terrain, between the various regions of the Kingdom, in addition to the immense size of the country. The findings revealed that the PHCs most affected were those located in rural and distant areas. In this context, the findings reveal that maintaining connectivity between the PHCs is considered to be challenge due to the terrain, poor infrastructure and the location of some PHCs. However, Study Two failed to examine the significant difference between rural and urban PHCs. It was difficult to determine which PHCs located in rural and which PHCs in urban places.

These findings are consistent with those of Lennon et al. (2017); Luna et al. (2014); Singh et al. (2012); and Whitacre and Williams (2015), who also detected poor infrastructure in rural and distant areas. In the same context, my findings and other similar studies show 
that infrastructure issues threatens the success of EHRS implementation projects (Ahmadian et al., 2014; Cresswell \& Sheikh, 2013; Fritz et al., 2015; Janamian et al., 2014; Luna et al., 2014), in particular developing countries (Ahmadian et al., 2014; Luna et al., 2014). Therefore, infrastructure development should be considered in the planning phase and the pre-implementation phase, as mentioned in the literature (see Chapter Two).

To overcome geographical challenges and ensure connectivity for all PHCs, my findings strongly recommended co-operating with third parties, such as local telecommunications companies, who can take responsibility for the provision of telecommunications and information technology. Problems in this area cannot be solved unilaterally and require the co-operation of all involved parties. This solution can contribute to developing appropriate infrastructure that facilitates the connectivity of all PHCs in SA. Subsequently, this connectivity will also assist in overcoming other challenges, such as technical support. Previous literature also identified other possible solutions to overcoming any challenges related to geography. For instance, the use of connections via mobile phones when the technical infrastructure is inadequate or does not exist (Jawhari et al., 2016; Khatun et al., 2015).

\subsubsection{Lack of systems interoperability}

Another technical obstacle, to emerge from the analysis of studies Three and Four, was the lack of EHRS interoperability, and some previous studies also point to this (Jha et al., 2009; (Ahmadian et al., 2014; Ajami \& Bagheri-Tadi, 2013; Janamian et al., 2014; Luna et al., 2014; Thakkar \& Davis, 2006). Although, previous literature found that interoperability issues are very costly (Luna et al., 2014; Rao et al., 2011), findings from Studies One and Four illustrated that FR contributed to EHRS interoperability (see Section 8.4.1). In large-scale projects, EHRS interoperability issues may become more complicated due to the multiplicity of the EHRS. As mentioned earlier in this chapter, the EHRS in PHCs in SA will be installed via three different vendors (See Chapter Seven). This multiplicity may produce interoperability issues that hinder the success of the project. Therefore, as illustrated in the literature (see Chapter Two), EHRS interoperability issues can be avoided when they are considered in the software selection 
process and other pre-implementation phase procedures, such as planning and readiness assessments (Alkraiji et al., 2013; Keshavjee et al., 2006).

Healthcare organisations can overcome interoperability issues through harmonisation of standards, with criteria and specifications being considered during software selection to ensure that all systems are compatible. Results, from studies One and Four, illustrated that CM helps to overcome this challenge through the standardisation of systems to ensure their compatibility, especially since the $\mathrm{MoH}$ is in the process of selecting three vendors. Hence, this should be carried out in the pre-implementation phase through the provision of Request for Proposal (RFP) to potential vendors when the project is launched for competition. Technically speaking, such a standard can help two or more systems to communicate with each other, as previous studies in the field of EHRS implementation have indicated (Begoyan, 2007; Kalra, 2006). In addition to these recommendations, pervious literature found that commercial EHRS are flexible and able to be integrated with other systems (Cresswell et al., 2013).

\subsubsection{Software selection challenges}

As illustrated in studies Three and Four, although the project team benefited from the FR granted to them and the CM that assisted the unification of decisions, software selection was a challenge. My findings revealed that, due to this challenge, no EHRS has been selected. This illustrates that FR are not always the solution when the decision is being made to purchase a new EHRS. One of the novel findings to emerge from the analysis of Study Four is that the PHCs in SA are different to PHCs in other countries, such as the UK, USA and Australia, in terms of workflow and structure (see Chapter Seven). These differences negatively affect the selection of an international system. The majority of international EHRS available on the market have been designed to fit with the most common workflow. These findings detected a relationship between PHC workflow and software selection. Therefore, those organisations with a rare or unique workflow need to consider workflow redesign or build their own systems.

The results from studies two and three also suggested that consideration should be given to system usability, efficiency and system security during software selection. These 
findings are in line with previous literature, which also suggested that consideration should be given to the above criteria (Hartzler et al., 2013; Keshavjee et al., 2006; Khajouei et al., 2011). To ensure that the system is easy to use, the $\mathrm{MoH}$ are planning to request a trial version of the software. This testing, with the co-operation of actual users of the EHRS, will measure their satisfaction with the system's usability. This result is consistent with those of previous studies conducted in the USA by Blumenthal (2011); and Corrao et al. (2010), who also recommend conducting a pilot test of the system before it is fully installed.

\subsubsection{EHRS end-user involvement}

Results (from studies Two, Three and Four) illustrated EHRS end-user involvement is one of the main points of disagreement between the project team and EHRS end-users. Although the project team stated that EHRS end-users have been involved in both planning and software selection (see Chapters Four and Seven), the majority of PHC staff and EHRS end-users argued that they were not involved in EHRS implementation. Moreover, EHRS end-users classified a lack of end-user involvement in decision-making and software selection as a major barrier to implementation. In the same context, the EHRS end-users stressed the importance of the consideration of end-user requirements and feedback. However, involving every individual in large-scale projects may be difficult, or even impossible. Despite the difficulties of involving all end-users in a largescale project, the project team took into consideration the negative consequences and are therefore are planning to develop a strategy to involve all stakeholders, whether directly or indirectly. The adoption of semi-centralised management and the appointment of champions and super-users were highly recommended to overcome end-users involvement issues in large-scale projects. Due to the importance of end-user involvement, the involvement mechanisms require further research, in particular for large-scale projects.

\subsubsection{Further recommendations to overcome the above barriers}

In addition to the recommendations mentioned previously in this chapter, this thesis provides several recommendations to overcome the above barriers. The findings 
illustrated that conducting studies and research can assist in overcoming some of barriers to the implementation of large-scale projects and find appropriate solutions to the challenges. In addition, conducting studies and research assists in determining the strengths and weaknesses that may influence EHRS implementation in PHCs in SA (see Chapter Seven). These studies and research found to be a useful tool to overcome the technical infrastructure issues such as lack of connectivity. Since the Saudi $\mathrm{MoH}$ has carried out several studies and associated research to assess their organisations readiness to the implementation of new EHRS, the future researcher can benefit from the findings of these studies by the utilisation of documentation analysis. In addition, documentation analysis can be carried out to the evaluations that conducted previously on the implemented EHRS (see Chapter Seven).

Another interesting recommendation, to emerge from the analysis of this thesis is, that of dividing the large-scale project into small projects, where each project can be implemented by a different vendor. This reinforces the success of the EHRS implementation and helps to avoid serious mistakes. Furthermore, policymakers need to consider the formulation of RFP to promote the implementation of a large-scale project. A well-designed RFP can assist in overcoming challenges, particularly in relation to software selection.

\subsection{Overall satisfaction with the EHRS implemented in PHCs in SA}

End-user's satisfaction, toward the implemented EHRS, was another factor of debate between the project team and the EHRS end-users. Although findings from Study Three show high level of satisfaction toward the EHRS implementation (see Chapter Six), findings form Study Four revealed a resistance toward the EHRS implementation (see Chapter Seven). Dissatisfaction with the implementation of the EHRS also documented among end-users in SA. Sixty-one percent of the participants claimed that they had abandoned the new EHRS and had gone back to the historical paper-based system (Alharthi et al., 2014). In contrast, in Arab Gulf Countries (AGCs) the EHRS end-users have recorded a high level of satisfaction towards the implementation and use of EHRS in secondary care (Alasmary et al., 2014; Bani-Issa et al., 2016). However, both Alasmary et al. (2014); and Alharthi et al. (2014) may face generalisability issues because the 
perceptions of other important parties, such as pharmacists, receptionists and laboratory technicians, were not considered in either study. This diversity requires further investigation, especially in AGCs.

Variable satisfaction towards the implementation of EHRS was also recorded in nonAGCs. While high levels of EHRS end-user' satisfaction have been recorded in several studies (Kim et al., 2016; Moody et al., 2004; Secginli et al., 2014), others have shown that EHRS end-users express moderate levels of satisfaction (Ahn et al., 2006; Rantz et al., 2011; Samoutis et al., 2007). In contrast, low levels of satisfaction or dissatisfaction have been expressed by EHRS end-users towards the use and adoption of the system, in other studies (e.g. Greenhalgh et al., 2010; Makam et al., 2013; Smith et al., 2005).

Finally, findings from Study Three also reveal a positive significant correlation between the perceived usefulness of the EHRS and the end-user's positive attitude toward the EHRS implementation. Similar to my findings, Abdekhoda et al. (2015) also documented a positive significant relationship between EHRS usefulness and end-user attitudes. On the other hand, Hamid and Cline (2013) reported a negative relationship between perceived usefulness and end-user satisfaction. This emphasises the importance of the system being beneficial, otherwise it may lead to the dissatisfaction of the end-users and on unsuccessful implementation.

The majority of findings in this section were obtained from Study Three. Therefore, it is worth noting that this study faces few weaknesses. Firstly, since EHRS end-user satisfaction has been evaluated based on the previously implemented EHRS, the findings may be negatively impacted by the current use of the system, with over three-quarters of the participants stating that they are not currently using the EHRS in their workplace. According to a member of the project team, some end-users have not used the EHRS for more than five years (see Chapter Seven). Secondly, although evaluation of the implemented EHRS in Study Four was made with consideration of the main factors that impact EHRS implementation (see Chapter Two), due to statistical issues, I was unable to test each factor individually. Some items represent the factors included in the data collection instrument, but they were not statistically reliable. For instance, although, a relationship has been documented between EHRS usability and perceived usefulness (Carr et al., 2010), due to reliability issues, I was unable to statistically examine this 
relationship. However, the utilised data collection instrument was statistically reliable. Secondly, the response rate to this study was lower (42.4\%), when compared with Study Two $(71.5 \%$ ) (see Chapter Five). This is most likely due to the questionnaire used in Study Three being longer than the questionnaire in Study Two. This indicates that shorter questionnaires are more favourable to participants and can thus improve the response rate.

\subsubsection{The impact of EHRS end-user demographic differences on their level of satisfaction}

It was hypothesised that participant demographics, such as age, gender, occupation, experience using an EHRS, and experience using computers, could influence end-user satisfaction with EHRS implementation (see Chapter Two). Contrary to expectations, with the exception of end-user occupation, this research did not find a significant difference between user demographics with regards to their satisfaction. With regards to the age of end-users, various studies have argued that older users show less satisfaction with an EHRS than younger users (Al Alawi et al., 2014; Decker et al., 2012; Duarte \& Azevedo, 2017; Hamid \& Cline, 2013; Holanda, do Carmo, et al., 2012; Kang \& Yoon, 2008; Moody et al., 2004; Urban et al., 2012; Ziefle \& Bay, 2005). In contrast, other research has revealed that older EHRS end-users were found to be more positive about EHRS implementation and usage than younger users (Alasmary et al., 2014; Khajouei et al., 2011; Khalifa \& Alswailem, 2015). Similar studies conducted in secondary care in SA also showed that older users expressed a higher level of satisfaction with EHRS (Alasmary et al., 2014; Khalifa \& Alswailem, 2015). This study, however, did not find any differences between the age group in terms of their satisfaction and attitudes towards EHRS implementation. This finding is consistent with those of Lai et al. (2004); Loomis et al. (2002); Morton and Wiedenbeck (2010); and Saleh et al. (2016), who also found age makes no significant difference to EHRS satisfaction. It is worth noting that the majority of the end-users were between 25-44 years old, and only four participants were between 55-74 years old.

Many researchers have identified a relationship between end-user gender and attitudes toward EHRS implementation. While Aldosari (2012); and Urban et al. (2012) reported that male users expressed higher levels of satisfaction compared with females, Al-Azmi 
et al. (2009); Hamid and Cline (2013); and Heyworth et al. (2012) found that males expressed a lower level of acceptance compared to females. The findings of this study identified that there was no significant difference between male and female, in terms of level of satisfaction (see Chapter Six). These findings are similar to those of Saleh et al. (2016), despite the differences in the targeted population, location of the study and data collection instruments.

While findings from Study Three found that computer experience has no impact on enduser satisfaction, previous studies have detected a correlation with previous computer experience (e.g. Alasmary et al., 2014; Khalifa \& Alswailem, 2015; Koivunen et al., 2008; Terry et al., 2009). They have identified that EHRS end-users, who have high levels of experience and skill in computers, expressed high levels of satisfaction toward EHRS implementation. It is worth noting that most EHRS end-users had more than one-year of experience using their computer at home, with only a small proportion stating that they had used a computer for less than one year (see Chapter Six).

According to Carayon et al. (2011); Chisolm et al. (2010); El-Kareh et al. (2009); Graetz et al. (2009); Holanda, Do Carmo E Sa, et al. (2012); Khajouei et al. (2011); Morton and Wiedenbeck (2010); and Sterns (2005), EHRS end-users, with previous experience of using an EHRS, are more likely to use a system than those without such experience. In contrast, other studies identified that EHRS end-user satisfaction decreased after a period of between six months and three years (ACoP, 2013; Heyworth et al., 2012; Laramee et $a l ., 2012)$. However, my findings suggest that there was no relationship between end-user experience, with the EHRS, and satisfaction with and attitudes towards it. The findings of this research also show no relationship between end-user experience at the workplace and satisfaction. These findings are in agreement with those of (Bouamrane \& Mair, 2013). However, Ahmadian et al. (2014); Zheng et al. (2010) showed that experience at work influenced EHRS satisfaction, where end-users with more experience expressed greater satisfaction compared with those with less experience.

Finally, findings from Study Three showed that physicians and radiologists expressed a less positive attitude towards EHRS implementation than laboratory technicians and pharmacists (see Table 11.8.1 in the Appendix B). It is worth noting that my research is one of only a few that includes radiologists and pharmacists in the target sample. Diverse 
results have been recorded in previous literature. While Khalifa and Alswailem (2015), who conducted a study in a tertiary care hospital in SA, found that pharmacists and physicians were less satisfied with the EHRS than other users, such as nurses and administrators, Bossen et al. (2013) showed that nurses and physiotherapists expressed higher satisfaction than physicians and medical secretaries.

Likewise, findings by Hoonakker et al. (2010); Sprague (2004); and Weiner et al. (1999) showed that nurses recorded higher satisfaction with the use of the EHRS than physicians. This contrasted with findings from other studies, which indicated that physicians were found to be more positive towards the use of the EHRS in comparison to nurses, and that they expressed a higher level of satisfaction (Alasmary et al., 2014; Bani-Issa et al., 2016; Kim et al., 2016; Lee et al., 1996). When considering other EHRS end-users, Laerum et al. (2004) documented that hospital medical secretaries were highly satisfied with the EHRS in comparison with physicians and nurses. The above statements are in contrast with data obtained from PHCs in Turkey by Secginli et al. (2014), who found that participant occupation makes no significant difference to level of satisfaction. This issue requires further investigation with EHRS end-users, and professions, other than physicians and nurses, need to be included.

\subsection{Summary}

Most of the findings from the research have been validated through the triangulation of sources. All the pre-specified objectives were addressed in at least two studies out of four. This chapter highlighted the novelty of some of the findings, which appear for the first time. During the description of the pre-implementation procedures, the findings revealed the significant role of consultations, in particular for large-scale projects which require cautious and more accurate procedures. The findings also identified that large-scale projects may face end-user involvement issues, due to the large number of such end-users. It has been reported that end-user involvement can be assured through the presence of champions, who can act as a bridge between end-users and the project team. The findings also suggested formulating RFP documents to facilitate software selection, which was also reported as a challenge for large-scale projects. To overcome this challenge, the project team decided to select more than one EHRS from three vendors, with 
consideration of systems interoperability. Since training and technical support are a challenge that may be faced by large-scale projects, they should be delivered with the support of the selected vendors.

The findings also showed a variable level of readiness of PHCs for EHRS implementation. While good readiness was recorded at the individual and organisational level, technological aspects, such as technical infrastructure, were documented to be at a lower level of readiness. Although many technical issues were detected as causes for failure of an implemented system, the Saudi MoH has taken a number of measures to increase the level of readiness of PHCs for EHRS implementation. For instance, they have contracted with TCs to equip the infrastructure of all PHCs, in particular those located in remote areas. Furthermore, one of the most interesting and unique findings was about the role of FR to enhance the implementation of a large-scale EHRS. Although the cost of EHRS implementation and lack of FR has been recorded as a barrier to implementation, for the first time, FR appears to be a facilitator of EHRS implementation. This thesis identified that FR has a positive impact on most factors that influence EHRS implementation, in addition to overcoming barriers to implementation.

Another novel finding of this thesis was the positive impact of CM on EHRS implementation in PHCs in SA. EHRS interoperability was the factor most influenced by $\mathrm{CM}$, as it has been illustrated that $\mathrm{CM}$ assists in overcoming interoperability issues by the unification of decisions, particularly in relation to software selection. However, CM can be risky, especially if individuals in decision-making positions are changed. Although the EHRS end-users expressed a high level of satisfaction with system usability, efficiency and usefulness, they were unhappy with the training and technical support. Therefore, the findings revealed that a lack of technical support and training led to end-user resistance to the system. The findings also revealed no significant differences in terms of demographic information and levels of satisfaction and readiness regarding EHRS implementation. 
9 Chapter Nine: Conclusion 


\subsection{What is known about EHRS implementation?}

The literature review, which investigated and evaluated EHRS implementation, revealed that all the factors influencing system implementation can be put into three categories: organisational, human, and technological. This categorisation is similar to the HOT-Fit framework (Yusof et al., 2008). A wide range of factors were determined through the literature review (e.g., planning, teamwork communication, project team selection, training, support, end-user satisfaction and acceptance, system usability and efficiency, and infrastructure). Demographic differences, such as age, gender and position, were documented as influential factors at the individual level. In addition, previous experience in using PCs, experience with an EHRS, and experience at the workplace were also found to have an impact on end-user satisfaction. Not only that, training, support, EHRS efficiency and usability also have a relationship to EHRS end-user satisfaction.

All the included literature contributed to identifying the obstacles and drivers directly associated with EHRS implementation. The greatest obstacles identified by the literature review were inadequate training and a lack of technical support at the organisational level, end-user resistance at the human level, and poor infrastructure at the technological level. Stakeholder involvement and the availability of champions at the organisational level, end-user satisfaction at the human level, and system efficiency at the technological level were identified as the biggest drivers in an EHRS implementation project.

\subsection{What this thesis adds}

This thesis contributes to the knowledge in research and practice in several ways. This is the first comprehensive investigation of the large-scale implementation of an EHRS in PHCs in SA. It has provided a deeper insight into the impact of CM and FR on largescale EHRS implementation. Prior to this study, it was difficult to make predictions about the impact of FR on EHRS implementation, although cost has been documented as a barrier. This thesis is also the only empirical investigation into the impact of $\mathrm{CM}$ on EHRS implementation in SA. Furthermore, the present study provides the first comprehensive assessment of Saudi PHCs readiness for the implementation of a largescale EHRS. Readiness assessments show that PHCs have higher readiness at the 
organisational and individual level than at the technical level. Moreover, the present study provides the first comprehensive insight into the previously implemented EHRS in Saudi PHCs, with an evaluation of the causes that led to the failure of the project. This thesis is one of the first attempts to thoroughly examine the provision of training and support in large-scale projects, especially in Saudi Arabian's PHCs.

This thesis also enhanced understanding about the relationship between individual demographic characteristics such as age, gender and occupation with level of readiness and satisfaction. The findings in this thesis provide a new understanding of the benefits of conducting effective communication among the project team in large-scale projects. Communication methods such as meetings, workshops and committees were found to be important to ensuring the success of large-scale EHRS implementation. Moreover, this thesis provides important insights into the role of consultations in planning and implementation.

This thesis highlighted novel findings about the facilitators and barriers that can directly affect the implementation of a large scale EHRS in SA's PHCs. Although a lack of technical support had been previously recorded to be a barrier to EHRS implementation, this is the first research to report an association between lack of technical support and failure of EHRS implementation in PHCs, in Saudi Arabia. Moreover, this thesis determined the large-scale of the project as one of the major challenges to implementation. The large-scale of the project can lead to several barriers such as lack of training, lack of EHRS end-user involvement and inadequate infrastructure. At the technical level, searching for an appropriate software can be a barrier when healthcare organisations such as PHCs in SA adopt a unique workflow. The individuality of the workflow can result in a mismatch between the available system and the existing workflow.

In addition to investigating barriers, this thesis provided unique recommendations on how to overcome barriers that accompany large-scale EHRS implementation, and presented a model for leading change and enhancing EHRS implementation in large-scale projects. For instance, coordination with TCs was found to be essential to preparing an adequate infrastructure. Another recommendation, to emerge from the findings, was the provision of training and technical support, which can be provided by the selected vendors. 
Moreover, to overcome any challenges that may accompany a large-scale project, it was highly recommended to conduct further research and studies to determine the main barriers to each particular project, as well as to conduct a pilot phase prior to any implementation to detect any barrier or issue before it occurs. Thereafter, the project team can produce an RFP for the potential vendors to ensure the appropriateness of their systems to be implemented and maintain the interoperability between different EHRS.

Initially, apart from the infrastructure and EHRS end-user involvement issues, this thesis determined Saudi PHCs to be at a good level of readiness. Software selection was identified as a critical step, and it was found that the project team were aware of this issue. Hence, several criteria were considered: usability, security, efficiency and usefulness. All the stakeholders' input and requirements should be considered during software selection. However, this thesis shows that large-scale projects may face stakeholder involvement issues due to the large number of stakeholders.

This thesis identified that, to enhance the EHRS implementation, a strategic implementation plan must involve several consultations that can be conducted at three levels: individual, organisational, and country. The findings identified that proper communication between all members of the project team can be assured via meetings, workshops and committees. In addition, distance communication such as video conferences and other media (e.g., email), facilitate communication between the project team in large-scale projects.

A comprehensive and clear strategic plan is key in facilitating the implementation of a large-scale EHRS and increasing the level of healthcare organisation readiness. Governmental financial and administrative support clearly facilitates EHRS implementation. Furthermore, an initial step towards increasing the level of individual readiness for EHRS implementation would be the introduction of an awareness campaign.

This thesis reported that the key drivers of large-scale EHRS implementation are FR, strong leadership and management, consultations, perceived usefulness, and user awareness and satisfaction. Consequently, this thesis revealed that what has been a barrier for other EHRS implementation projects, is not so for Saudi PHCs. For example, although the cost of EHRS implementation has been recorded to be a barrier in many projects, the 
implementation of an EHRS in Saudi PHCs is not subject to financial constraints. On the other hand, project scale, staff changes (e.g., senior managers and ministers), geographical challenges, poor infrastructure and a lack of connectivity were the greatest obstacles to EHRS implementation.

Patient confidentiality and privacy were at the top of the project team and policy-makers' priorities. This was addressed by selecting secure software and the formulation of policies, laws and regulations to protect patient data; all were considered to maintain the privacy and confidentiality of patient data. Therefore, this thesis identified that regulations and policies, including providing privileges to the users, a non-disclosure agreement document, and monitoring transactions in the EHRS, were essential to ensuring privacy and confidentiality.

This thesis found that $\mathrm{CM}$ can assist in overcoming challenges regarding software selection, interoperability, project team communication and other challenges which accompany larger-scale projects. FR facilitates training, support, software selection, and preparation of adequate infrastructure. However, this thesis showed that CM may negatively influence EHRS implementation and threaten the success of the project, especially when key individuals are changed.

One of the most vital solutions identified by this thesis was the division of any large-scale project into several zones that can then be implemented by more than one vendor. This thesis identified that, one of the solutions to avoiding failure and ensuring a high level of readiness of PHCs, is to pilot the system prior to the actual implementation and for a clear RFP to be produced. These theoretical insights, although based on EHRS implementation in Saudi PHCs, provide potentially transferable lessons to similar projects in other countries around the world, regardless of the size of the project or even the size of the healthcare organisation. For example, FR are crucial to the success of EHRS implementation. 


\subsection{Recommendations for future research}

Further research is needed to examine the readiness of Saudi PHCs in rural and distant areas, areas which may face greater infrastructure readiness issues compared with urban PHCs. This thesis assessed the readiness of the Saudi PHCs for EHRS implementation, however, there is a need for future studies to assess secondary care and other private healthcare organisation readiness also, especially those in SA that are yet to implement an EHRS. Moreover, based on the limitation mentioned in Chapter Eight, future researcher may need to consider using documentation analysis to assess the PHCs readiness to the EHRS implementation.

Comparative studies between PHCs in SA and PHCs in other developing countries, especially AGCs, need to be carried out to determine the differences in the level of readiness for EHRS implementation. AGCs may share some of the characteristics of SA, such as the type of project management and the level of FR, therefore, further studies need to be conducted to examine the impact of CM and FR on EHRS implementation in other AGCs, or in other developing countries.

Although the findings of this thesis identified that CM had a positive impact on EHRS implementation in PHCs in SA, some of the determined obstacles to EHRS implementation in Saudi PHCs can be directly linked to CM. For instance, the previous EHRS failed, due to the replacement of key people who centrally managed the project. Therefore, further studies are needed to investigate the relationship between EHRS project failure and CM. Moreover, further research needs to be carried out to examine the impact of CM on an EHRS implementation in secondary care setting, especially largescale projects.

Finally, searching through the previous literature, the work demonstrated that only one study has been conducted to measure the EHRS adoption rate in the eastern province of SA. Therefore, state-wide research is required to measure the EHRS adoption rate in both secondary and primary care in SA. Although I have made several recommendations to enhance the implementation of a large-scale EHRS in PHCs, specific research to explore this particular project is still needed. 


\section{References}

Aarts, J., Doorewaard, H., \& Berg, M. (2004). Understanding implementation: the case of a computerized physician order entry system in a large Dutch university medical center. J Am Med Inform Assoc, 11(3), 207-216. doi:10.1197/jamia.M1372

Abdekhoda, M., Ahmadi, M., Gohari, M., \& Noruzi, A. (2015). The effects of organizational contextual factors on physicians' attitude toward adoption of Electronic Medical Records. J Biomed Inform, 53, 174-179. doi:10.1016/j.jbi.2014.10.008

ACoP, American College of Physicians. (2013). Survey of Clinicians: User satisfaction with electronic health records has decreased since 2010. Retrieved from https://www.acponline.org/acp-newsroom/survey-of-clinicians-user-satisfactionwith-electronic-health-records-has-decreased-since-2010

Adler, K. G. (2005). How to select an electronic health record system. Fam Pract Manag, 12(2), 55-62.

Adler, K. G. (2007). How to successfully navigate your EHR implementation. Fam Pract Manag, 14(2), 33-39.

Ahmadian, L., Khajouei, R., Nejad, S. S., Ebrahimzadeh, M., \& Nikkar, S. E. (2014). Prioritizing barriers to successful implementation of hospital information systems. J Med Syst, 38(12), 151. doi:10.1007/s10916-014-0151-9

Ahmadian, L., Nejad, S. S., \& Khajouei, R. (2015). Evaluation methods used on health information systems (HISs) in Iran and the effects of HISs on Iranian healthcare: a systematic review. Int J Med Inform, 84(6), 444-453. doi:10.1016/j.ijmedinf.2015.02.002

Ahn, T. S., Park, I. S., You, O. S., Shin, H. J., Woo, K. S., \& Jo, E. M. (2006). Nurses' perceptions of and attitudes toward an electronic medical record system at Seoul National University Hospital. Stud Health Technol Inform, 122, 851.

Ajami, S., \& Arab-Chadegani, R. (2013). Barriers to implement Electronic Health Records (EHRs). Materia Socio-Medica, 25(3), 213-215. doi:10.5455/msm.2013.25.213-215

Ajami, S., \& Bagheri-Tadi, T. (2013). Barriers for Adopting Electronic Health Records (EHRs) by Physicians. Acta Inform Med, 21(2), 129-134. doi:10.5455/aim.2013.21.129-134 
Ajami, S., Ketabi, S., Isfahani, S. S., \& Heidari, A. (2011). Readiness assessment of electronic health records implementation. Acta Inform Med, 19(4), 224-227. doi:10.5455/aim.2011.19.224-227

Al-Azmi, S. F., Al-Enezi, N., \& Chowdhury, R. I. (2009). Users' attitudes to an electronic medical record system and its correlates: a multivariate analysis. HIM $J, 38(2), 33-40$.

Al-Yousuf, M., Akerele, T. M., \& Al-Mazrou, Y. Y. (2002). Organization of the Saudi health system. East Mediterr Health J, 8(4-5), 645-653.

Al Alawi, S., Al Dhaheri, A., Al Baloushi, D., Al Dhaheri, M., \& Prinsloo, E. (2014). Physician user satisfaction with an electronic medical records system in primary healthcare centres in Al Ain: a qualitative study. BMJ Open, 4(11). doi:10.1136/bmjopen-2014-005569

Alasmary, M., El Metwally, A., \& Househ, M. (2014). The association between computer literacy and training on clinical productivity and user satisfaction in using the electronic medical record in Saudi Arabia. J Med Syst, 38(8), 69. doi:10.1007/s 10916-014-0069-2

Aldosari, B. (2012). User acceptance of a picture archiving and communication system (PACS) in a Saudi Arabian hospital radiology department. BMC Medical Informatics and Decision Making, 12(1), 44. doi:10.1186/1472-6947-12-44

Aldosari, Bakheet. (2014). Rates, levels, and determinants of electronic health record system adoption: A study of hospitals in Riyadh, Saudi Arabia. Int J Med Inform, 83(5), 330-342. doi:https://doi.org/10.1016/j.ijmedinf.2014.01.006

Alghamdi, M. G., \& Urden, L. D. (2016). Transforming the nursing profession in Saudi Arabia. J Nurs Manag, 24(1), E95-E100. doi:10.1111/jonm.12301

Alhamad, B. , \& Aladwan, R. (2019). Balancing centralization and decentralization management at University of Bahrain. Quality Assurance in Education(2), 237. doi:10.1108/QAE-01-2018-0005

Alharthi, H., Youssef, A., Radwan, S., Al-Muallim, S., \& Zainab, A. (2014). Physician satisfaction with electronic medical records in a major Saudi Government hospital. Journal of Taibah University Medical Sciences, 9(3), 213-218. doi:https://doi.org/10.1016/j.jtumed.2014.01.004

Alkraiji, A., Jackson, T., \& Murray, I. (2013). Barriers to the widespread adoption of health data standards: an exploratory qualitative study in tertiary healthcare organizations in saudi arabia. J Med Syst, 37(2), 9895. doi:10.1007/s10916-0129895-2 
Allen, M. J., Kaufman, D. M., Barrett, A., Paterson, G., Sargeant, J., \& McLeod, R. (2000). Self-reported effects of computer workshops on physicians' computer use. J Contin Educ Health Prof, 20(1), 20-26. doi:10.1002/chp.1340200105

Almalki, M., Fitzgerald, G., \& Clark, M. (2011). Health care system in Saudi Arabia: an overview. East Mediterr Health J, 17(10), 784-793.

Alsahafi, Y.A. (2012). Studies of EHR Implementation and Operation in Different Countries with Particular Reference to Saudi Arabia. Auckland: Massey University, Albany.

Altuwaijri, M. M. (2008). Electronic-health in Saudi Arabia. Just around the corner? Saudi Medical Journal, 29(2), 171-178.

Altuwaijri, M. M. (2011, 15-17 Oct. 2011). Achieving excellence in Electronic Health Record deployment in Middle East hospitals. Paper presented at the Biomedical Engineering and Informatics (BMEI), 2011 4th International Conference on.

Altwaijiri, M., \& Aldosari, B. (2008). Health Informatics Master Program at King Saud bin Abdulaziz University for Health Sciences, Riyadh, Saudi Arabia. Yearb Med Inform, 145-149.

Amatayakul, M. (2005). EHR? Assess readiness first. Healthc Financ Manage, 59(5), $112-113$.

Amatayakul, M. (2007). Do you plan not to achieve your EHR ROI? Healthc Financ Manage, 61(11), 146-147.

Ammenwerth, E., Iller, C., \& Mahler, C. (2006). IT-adoption and the interaction of task, technology and individuals: a fit framework and a case study. BMC Medical Informatics and Decision Making, 6, 3-3. doi:10.1186/1472-6947-6-3

Ancker, J. S., Singh, M. P., Thomas, R., Edwards, A., Snyder, A., Kashyap, A., \& Kaushal, R. (2013). Predictors of success for electronic health record implementation in small physician practices. Appl Clin Inform, 4(1), 12-24. doi:10.4338/ACI-2012-09-RA-0033

Anderson, J.G., \& Aydin, C. (2006). Evaluating the Organizational Impact of Health Care Information Systems (Vol. 1). New York: Springer.

Arvanitis, T. N. (2014). Semantic interoperability in healthcare. In Integration Information Technology and Management for Quality of Care (pp. 5-8). Amsterdam: IOS press. 
Ash, J. S., \& Bates, D. W. (2005). Factors and forces affecting EHR system adoption: report of a 2004 ACMI discussion. J Am Med Inform Assoc, 12(1), 8-12. doi:10.1197/jamia.M1684

Ash, J. S., Fournier, L., Stavri, P. Z., \& Dykstra, R. (2003). Principles for a successful computerized physician order entry implementation. AMIA Annu Symp Proc, 3640. doi:D030002698 [pii]

Ash, J. S., Sittig, D. F., Guappone, K., Dykstra, R., Richardson, J. E., Wright, A., . . . Middleton, B. (2012). Recommended practices for computerized clinical decision support and knowledge management in community settings: a qualitative study. BMC Medical Informatics and Decision Making, 12(1), 6.

Ash, JS, Sittig, DF, Poon, EG, Guappone, K, Campbell, E, \& Dykstra, RH. (2007). The extent and important of unintended consequences related to computerized provider order entry. J Am Med Inform Assoc, 14(4), 415 - 423.

Atherton, J. . (2011). Development of the Electronic Health Record. AMA Journal of Ethics, 13, 186-189.

Bah, S., Alharthi, H., El Mahalli, A., Jabali, A., Al-Qahtani, M., \& Al-kahtani, N. (2011). Annual Survey on the Level and Extent of Usage of Electronic Health Records in Government-related Hospitals in Eastern Province, Saudi Arabia. Perspectives in Health Information Management / AHIMA, American Health Information Management Association, 8(Fall), $1 \mathrm{~b}$.

Bahaadinbeigy, K., Yogesan, K., \& Wootton, R. (2010). MEDLINE versus EMBASE and CINAHL for telemedicine searches. Telemed J E Health, 16(8), 916-919. doi:10.1089/tmj.2010.0046

Bani-Issa, W., Al Yateem, N., Al Makhzoomy, I. K., \& Ibrahim, A. (2016). Satisfaction of health-care providers with electronic health records and perceived barriers to its implementation in the United Arab Emirates. Int J Nurs Pract, 22(4), 408416. doi:10.1111/ijn.12450

Barbour, R. (2013). Introducing Qualitative Research: A Student's Guide. United Kingdom: SAGE Publications.

Bardhan, I., \& Thouin, M. (2013). Health information technology and its impact on the quality and cost of healthcare delivery. Decision Support Systems, 55(2), 438449. doi:http://dx.doi.org/10.1016/j.dss.2012.10.003

Barrows, R. C., Jr., \& Clayton, P. D. (1996). Privacy, confidentiality, and electronic medical records. J Am Med Inform Assoc, 3(2), 139-148. 
Bates, D. W., Ebell, M., Gotlieb, E., Zapp, J., \& Mullins, H. C. (2003). A proposal for electronic medical records in U.S. primary care. J Am Med Inform Assoc, 10(1), $1-10$.

Begoyan, A. (2007). An overview of interoperability standards for electronic health records. Paper presented at the Integrated Design and Process Technology, USA.

Berner, E. S., Detmer, D. E., \& Simborg, D. (2005). Will the wave finally break? A brief view of the adoption of electronic medical records in the United States. $J$ Am Med Inform Assoc, 12(1), 3-7. doi:10.1197/jamia.M1664

Biruk, S., Yilma, T., Andualem, M., \& Tilahun, B. (2014). Health Professionals' readiness to implement electronic medical record system at three hospitals in Ethiopia: a cross sectional study. BMC Med Inform Decis Mak, 14, 115. doi:10.1186/s 12911-014-0115-5

Blumenthal , D. (2011). Implementation of the Federal Health Information Technology Initiative. New England Journal of Medicine, 365(25), 2426-2431. doi:doi:10.1056/NEJMsr1112158

Boffa, D. P., \& Pawola, L. M. (2006). Identification and conceptualization of nurse super users. J Healthc Inf Manag, 20(4), 60-68.

Boonstra, A., \& Broekhuis, M. (2010). Barriers to the acceptance of electronic medical records by physicians from systematic review to taxonomy and interventions. BMC Health Serv Res, 10, 231. doi:10.1186/1472-6963-10-231

Boonstra, A., Versluis, A., \& Vos, J. F. (2014). Implementing electronic health records in hospitals: a systematic literature review. BMC Health Serv Res, 14, 370. doi:10.1186/1472-6963-14-370

Bossen, C. (2007). Test the artefact--develop the organization. The implementation of an electronic medication plan. Int J Med Inform, 76(1), 13-21. doi:10.1016/j.ijmedinf.2006.01.001

Bossen, C., Jensen, L. G., \& Udsen, F. W. (2013). Evaluation of a comprehensive EHR based on the DeLone and McLean model for IS success: approach, results, and success factors. Int J Med Inform, 82(10), 940-953. doi:10.1016/j.ijmedinf.2013.05.010

Bouamrane, M. M., \& Mair, F. (2013). A study of general practitioners' perspectives on electronic medical records systems in NHSScotland. BMC Medical Informatics and Decision Making, 13(1), 58-58. doi:10.1186/1472-6947-13-58 
Bowling, A. (2009). Research methods in health: investigating health and health services (3rd edition ed.). Maidenhead, Berkshire, England: McGraw Hill/Open University Press.

Boyer, L., Samuelian, J. C., Fieschi, M., \& Lancon, C. (2010). Implementing electronic medical records in a psychiatric hospital: A qualitative study. Int J Psychiatry Clin Pract, 14(3), 223-227. doi:10.3109/13651501003717243

Braun, V., \& Clarke, V. (2006). Using thematic analysis in psychology. Qualitative Research in Psychology, 3(2), 77-101. doi:10.1191/1478088706qp063oa

Bryman, A. (2012). Social Research Methods (4th ed.). United Kingdom OUP Oxford.

Buttle, Francis. (1996). SERVQUAL: review, critique, research agenda. European Journal of Marketing, 30, 8-32. doi:10.1108/03090569610105762

Calnan, M., Katsouyiannopoulos, V., Ovcharov, V. K., Prokhorskas, R., Ramic, H., \& Williams, S. (1994). Major determinants of consumer satisfaction with primary care in different health systems. Fam Pract, 11(4), 468-478.

Car, J., Black, A., Anandan, C. , Cresswell, K. , Pagliari, C., McKinstry, B. , . . . Sheikh, A. . (2008). The Impact of eHealth on the Quality \& Safety of Healthcare. Retrieved from https://www1.imperial.ac.uk/resources/32956FFCBD76-47B7-94D2-FFAC56979B74/

Carayon, P., Cartmill, R., Blosky, M. A., Brown, R., Hackenberg, M., Hoonakker, P., . . . Walker, J. M. (2011). ICU nurses' acceptance of electronic health records. J Am Med Inform Assoc, 18(6), 812-819. doi:10.1136/amiajnl-2010-000018

Carayon, P., Smith, P., Hundt, A. S., Kuruchittham, V., \& Li, Q. (2009). Implementation of an electronic health records system in a small clinic: the viewpoint of clinic staff AU - Carayon, Pascale. Behaviour \& Information Technology, 28(1), 5-20. doi:10.1080/01449290701628178

Carayon, P., Wetterneck, T. B., Rivera-Rodriguez, A. J., Hundt, A. S., Hoonakker, P., Holden, R., \& Gurses, A. P. (2014). Human factors systems approach to healthcare quality and patient safety. Appl Ergon, 45(1), 14-25. doi:10.1016/j.apergo.2013.04.023

Carr, A., Zhang, M., Klopping, I., \& Min, H. (2010). RFID Technology: Implications for Healthcare Organizations. American Journal of Business, 25(2), 25-40. doi:doi:10.1108/19355181201000008

Chao, W. C., Hu, H., Ung, C. O., \& Cai, Y. (2013). Benefits and challenges of electronic health record system on stakeholders: a qualitative study of outpatient physicians. J Med Syst, 37(4), 9960. doi:10.1007/s10916-013-9960-5 
Chaudhry, B., Wang, J., Wu, S., Maglione, M., Mojica, W., Roth, E., . . Shekelle, P. G. (2006). Systematic review: impact of health information technology on quality, efficiency, and costs of medical care. Annals of Internal Medicine, 144(10), 742752.

Chen, C., Garrido, T., Chock, D., Okawa, G., \& Liang, L. (2009). The Kaiser Permanente Electronic Health Record: Transforming And Streamlining Modalities Of Care. Health Affairs, 28(2), 323-333. doi:10.1377/hlthaff.28.2.323

Cheriff, A. D., Kapur, A. G., Qiu, M., \& Cole, C. L. (2010). Physician productivity and the ambulatory EHR in a large academic multi-specialty physician group. Int $J$ Med Inform, 79(7), 492-500. doi:10.1016/j.ijmedinf.2010.04.006

Cherry, B. (2011). Assessing organizational readiness for electronic health record adoption in long-term care facilities. J Gerontol Nurs, 37(10), 14-19. doi:10.3928/00989134-20110831-06

Chisolm, D. J., Purnell, T. S., Cohen, D. M., \& McAlearney, A. S. (2010). Clinician perceptions of an electronic medical record during the first year of implementaton in emergency services. Pediatr Emerg Care, 26(2), 107-110. doi:10.1097/PEC.0b013e3181ce2f99

CITC, Communications and Information Technology Commission. (2005). Saudi Arabia-towards the Information Society. Retrieved from http://opencourseware.kfupm.edu.sa/colleges/cim/acctmis/mis345/files\%5C5Handouts_KSA-to-information-society.pdf

Clarke, M. A., Belden, J. L., Koopman, R. J., Steege, L. M., Moore, J. L., Canfield, S. M., \& Kim, M. S. (2013). Information needs and information-seeking behaviour analysis of primary care physicians and nurses: a literature review. Health Info Libr J, 30(3), 178-190. doi:10.1111/hir.12036

Corrao, N., Robinson, A., Swiernik, M., \& Naeim, A. (2010). Importance of Testing for Usability When Selecting and Implementing an Electronic Health or Medical Record System. Journal of Oncology Practice, 6(3), 120-124. doi:10.1200/jop.200017

Crampton, P., Jatrana, S., Lay-Yee, R., \& Davis, P. (2007). Exposure to primary medical care in New Zealand: number and duration of general practitioner visits. N Z Med J, 120(1256), U2582.

Cresswell, K. M., Bates, D. W., \& Sheikh, A. (2013). Ten key considerations for the successful implementation and adoption of large-scale health information technology. J Am Med Inform Assoc, 20(e1), e9-e13. doi:10.1136/amiajnl-2013001684 
Cresswell, K. M., Worth, A., \& Sheikh, A. (2012). Comparative case study investigating sociotechnical processes of change in the context of a national electronic health record implementation. Health Informatics J, 18(4), 251-270. doi:10.1177/1460458212445399

Cresswell, K., \& Sheikh, A. (2013). Organizational issues in the implementation and adoption of health information technology innovations: an interpretative review. Int J Med Inform, 82(5), e73-86. doi:10.1016/j.ijmedinf.2012.10.007

Creswell, J.W. (2014). Research Design: Qualitative, Quantitative, and Mixed Methods Approaches. LINCOLN, USA: SAGE Publications.

Cucciniello, M., Lapsley, I., Nasi, G., \& Pagliari, C. (2015). Understanding key factors affecting electronic medical record implementation: a sociotechnical approach. BMC Health Serv Res, 15, 268. doi:10.1186/s12913-015-0928-7

Daniel, J. (2011). Sampling Essentials: Practical Guidelines for Making Sampling Choices (1st ed.). Thousand Oaks, CA: SAGE Publications.

Davis, F. (1989). Perceived Usefulness, Perceived Ease of Use, and User Acceptance of Information Technology. MIS Quarterly, 13(3), 319-340. doi:10.2307/249008

Dawson, C. (2009). Introduction to Research Methods: A Practical Guide for Anyone Undertaking a Research Project (5th ed. Vol. 1). United Kingdom: Hachette

de la Torre, I., Gonzalez, S., \& Lopez-Coronado, M. (2012). Analysis of the EHR systems in Spanish Primary Public Health System: the lack of interoperability. $J$ Med Syst, 36(5), 3273-3281. doi:10.1007/s 10916-011-9818-7

Decker, S., Jamoom, E., \& Sisk, J. (2012). Physicians In Nonprimary Care And Small Practices And Those Age 55 And Older Lag In Adopting Electronic Health Record Systems. Health Affairs. doi:10.1377/hlthaff.2011.1121

DesRoches, C. M., Campbell, E. G., Rao, S. R., Donelan, K., Ferris, T. G., Jha, A., . . Blumenthal, D. (2008). Electronic health records in ambulatory care--a national survey of physicians. N Engl J Med, 359(1), 50-60. doi:10.1056/NEJMsa0802005

DesRoches, C. M., Worzala, C., Joshi, M. S., Kralovec, P. D., \& Jha, A. K. (2012). Small, nonteaching, and rural hospitals continue to be slow in adopting electronic health record systems. Health Aff (Millwood), 31(5), 1092-1099. doi:10.1377/hlthaff.2012.0153

Deutsch, E., Duftschmid, G., \& Dorda, W. (2010). Critical areas of national electronic health record programs-Is our focus correct? Int J Med Inform, 79(3), 211-222. doi:DOI 10.1016/j.ijmedinf.2009.12.002 
Devine, E. B., Patel, R., Dixon, D. R., \& Sullivan, S. D. (2010). Assessing attitudes toward electronic prescribing adoption in primary care: a survey of prescribers and staff. Inform Prim Care, 18(3), 177-187.

Dhivyadeepa, E. (2015). Sampling Techniques in Educational Research (1st ed. Vol. 1). USA: Laxmi Book Publication.

Doebbeling, B. N., Chou, A. F., \& Tierney, W. M. (2006). Priorities and strategies for the implementation of integrated informatics and communications technology to improve evidence-based practice. J Gen Intern Med, 21 Suppl 2, S50-57. doi:10.1111/j.1525-1497.2006.00363.x

Duarte, J. G., \& Azevedo, R. S. (2017). Electronic health record in the internal medicine clinic of a Brazilian university hospital: Expectations and satisfaction of physicians and patients. Int J Med Inform, 102, 80-86. doi:10.1016/j.ijmedinf.2017.03.007

Dzimbiri, L.B. (2009). Organisation and Management Theories: an African Focus: Integrating Structure, People, Processes and the Environment for Human Happiness. Zomba, Malawi: Cuvillier Verlag.

Ekvall, G. (1996). Organizational climate for creativity and innovation. European Journal of Work and Organizational Psychology, 5(1), 105-123. doi:10.1080/13594329608414845

El-Kareh, R., Gandhi, T. K., Poon, E. G., Newmark, L. P., Ungar, J., Lipsitz, S., \& Sequist, T. D. (2009). Trends in primary care clinician perceptions of a new electronic health record. J Gen Intern Med, 24(4), 464-468. doi:10.1007/s11606009-0906-z

El Mahalli, A. (2015). Adoption and Barriers to Adoption of Electronic Health Records by Nurses in Three Governmental Hospitals in Eastern Province, Saudi Arabia. Perspect Health Inf Manag, 12, 1f.

Escobar-Rodriguez, T., \& Bartual-Sopena, L. (2013). The roles of users personal characteristics and organisational support in the attitude towards using ERP systems in a Spanish public hospital. HIM J, 42(1), 18-28.

Fernandez-Aleman, J. L., Senor, I. C., Lozoya, P. A., \& Toval, A. (2013). Security and privacy in electronic health records: a systematic literature review. $J$ Biomed Inform, 46(3), 541-562. doi:10.1016/j.jbi.2012.12.003

Field, A. (2013). Discovering Statistics Using IBM SPSS Statistics. United Kingdom: SAGE Publications. 
Fritz, F., Tilahun, B., \& Dugas, M. (2015). Success criteria for electronic medical record implementations in low-resource settings: a systematic review. J Am Med Inform Assoc, 22(2), 479-488. doi:10.1093/jamia/ocu038

Fullerton, C., Aponte, P., Hopkins, R., Bragg, D., \& Ballard, D. J. (2006). Lessons learned from pilot site implementation of an ambulatory electronic health record. Proc (Bayl Univ Med Cent), 19(4), 303-310.

Gagnon, M., Attieh, R., Ghandour, E., Legare, F., Ouimet, M., Estabrooks, C., \& Grimshaw, J. (2014). A Systematic Review of Instruments to Assess Organizational Readiness for Knowledge Translation in Health Care. PLoS ONE, 9(12), e114338. doi:10.1371/journal.pone.0114338

Gagnon, M., Desmartis, M., Labrecque, M., Légaré, F., Lamothe, L., Fortin, J., . . . Duplantie, J. (2010). Implementation of an electronic medical record in family practice: a case study. Informatics In Primary Care, 18(1), 31-40.

Gagnon, M. P., Desmartis, M., Labrecque, M., Car, J., Pagliari, C., Pluye, P., . . . Legare, F. (2012). Systematic review of factors influencing the adoption of information and communication technologies by healthcare professionals. $J$ Med Syst, 36(1), 241-277. doi:10.1007/s10916-010-9473-4

Gagnon, M. P., Desmartis, M., Labrecque, M., Legare, F., Lamothe, L., Fortin, J. P., . . . Duplantie, J. (2010). Implementation of an electronic medical record in family practice: a case study. Inform Prim Care, 18(1), 31-40.

Gagnon, M. P., Ghandour el, K., Talla, P. K., Simonyan, D., Godin, G., Labrecque, M., ... Rousseau, M. (2014). Electronic health record acceptance by physicians: testing an integrated theoretical model. J Biomed Inform, 48, 17-27. doi:10.1016/j.jbi.2013.10.010

Gagnon, M. P., Nsangou, E. R., Payne-Gagnon, J., Grenier, S., \& Sicotte, C. (2014). Barriers and facilitators to implementing electronic prescription: a systematic review of user groups' perceptions. J Am Med Inform Assoc, 21(3), 535-541. doi:10.1136/amiajnl-2013-002203

Gagnon, M. P., Ouimet, M., Godin, G., Rousseau, M., Labrecque, M., Leduc, Y., \& Ben Abdeljelil, A. (2010). Multi-level analysis of electronic health record adoption by health care professionals: a study protocol. Implement Sci, 5, 30. doi:10.1186/1748-5908-5-30

Gagnon, M., Payne-Gagnon, J., Breton, E., Fortin, J., Khoury, L., Dolovich, L. R., . . . Archer, N. (2016). Adoption of Electronic Personal Health Records in Canada: Perceptions of Stakeholders. International Journal of Health Policy and Management, 5(7), 425-433. doi:10.15171/ijhpm.2016.36 
Gajanayake, R., Sahama, T., \& Iannella, R. (2013, 9-12 Oct. 2013). The role of perceived usefulness and attitude on electronic health record acceptance. Paper presented at the 2013 IEEE 15th International Conference on e-Health Networking, Applications and Services (Healthcom 2013).

Gascon, F., Herrera, I., Vazquez, C., Jimenez, P., Jimenez, J., Real, C., \& Perez, F. (2013). Electronic health record: Design and implementation of a lab test request module. Int J Med Inform. doi:10.1016/j.ijmedinf.2013.03.006

Ghazisaeidi, M., Ahmadi, M., Sadoughi, F., \& Safdari, R. (2014). A roadmap to preimplementation of electronic health record: the key step to success. Acta Inform Med, 22(2), 133-138. doi:10.5455/aim.2014.22.133-138

Ghuman, K. (2010). Management: Concepts, Practice \& Cases (1st ed.). India: McGraw-Hill Education (India) Pvt Limited.

Godoy, D., \& Soares, A. (2017). Electronic health record in the internal medicine clinic of a Brazilian university hospital: Expectations and satisfaction of physicians and patients. Int J Med Inform, 102(Supplement C), 80-86. doi:https://doi.org/10.1016/j.ijmedinf.2017.03.007

Gordon, S. H., Baier, R. R., \& Gardner, R. L. (2015). Primary Care Physicians' Use of Electronic Health Records in Rhode Island: 2009-2014. R I Med J (2013), 98(10), 29-32.

Gosden, T., Forland, F., Kristiansen, I. S., Sutton, M., Leese, B., Giuffrida, A., . . . Pedersen, L. (2000). Capitation, salary, fee-for-service and mixed systems of payment: effects on the behaviour of primary care physicians. Cochrane Database Syst Rev(3), CD002215. doi:10.1002/14651858.CD002215

Gottschalk, A., \& Flocke, S. A. (2005). Time spent in face-to-face patient care and work outside the examination room. Ann Fam Med, 3(6), 488-493. doi:10.1370/afm.404

Graetz, I., Reed, M., Rundall, T., Bellows, J., Brand, R., \& Hsu, J. (2009). Care coordination and electronic health records: connecting clinicians. AMIA Annu Symp Proc, 2009, 208-212.

Green, L. A., Fryer, G. E., Jr., Yawn, B. P., Lanier, D., \& Dovey, S. M. (2001). The ecology of medical care revisited. N Engl J Med, 344(26), 2021-2025. doi:10.1056/NEJM200106283442611

Greenhalgh, T., Potts, H. W. W., Wong, G., Bark, P., \& Swinglehurst, D. (2009). Tensions and Paradoxes in Electronic Patient Record Research: A Systematic Literature Review Using the Meta-narrative Method. Milbank Quarterly, 87(4), 729-788. 
Greenhalgh, T., Stramer, K., Bratan, T., Byrne, E., Mohammad, Y., \& Russell, J. (2008). Introduction of shared electronic records: multi-site case study using diffusion of innovation theory. BMJ, 337, a1786. doi:10.1136/bmj.a1786

Greenhalgh, T., Stramer, K., Bratan, T., Byrne, E., Russell, J., \& Potts, H. W. W. (2010). Adoption and non-adoption of a shared electronic summary record in England: a mixed-method case study. BMJ, 340. doi:10.1136/bmj.c3111

Guest, G., MacQueen, K.M., \& Namey, E.E. (2011). Applied Thematic Analysis. Pennsylvania, USA: SAGE Publications.

Gugerty, B., Maranda, M., \& Rook, D. (2006). The clinical information system implementation evaluation scale. Stud Health Technol Inform, 122, 621-625.

Hamid, F., \& Cline, T. (2013). Providers' Acceptance Factors and their Perceived Barriers to Electronic Health Record (EHR) Adoption. Journal of Nursing Informatics, 17(3).

Handel, D. A., \& Hackman, J. L. (2010). Implementing electronic health records in the emergency department. J Emerg Med, 38(2), 257-263. doi:10.1016/j.jemermed.2008.01.020

Harman, J. S., Rost, K. M., Harle, C. A., \& Cook, R. L. (2012). Electronic medical record availability and primary care depression treatment. J Gen Intern Med, 27(8), 962-967. doi:10.1007/s11606-012-2001-0

Hartzler, A., McCarty, C. A., Rasmussen, L. V., Williams, M. S., Brilliant, M., Bowton, E. A., . . Trinidad, S. B. (2013). Stakeholder engagement: a key component of integrating genomic information into electronic health records. Genet Med, 15(10), 792-801. doi:10.1038/gim.2013.127

Hasanain, R., Vallmuur, K., \& Clark, M. (2014). Progress and challenges in the implementation of Electronic Medical Records in Saudi Arabia : a systematic review. Health Informatics - An International Journal, 3(2). doi:10.5121/hiij.2014.3201

Hayrinen, K., Saranto, K., \& Nykanen, P. (2008). Definition, structure, content, use and impacts of electronic health records: a review of the research literature. Int $J$ Med Inform, 77(5), 291-304. doi:10.1016/j.ijmedinf.2007.09.001

Heyworth, L., Zhang, F., Jenter, C. A., Kell, R., Volk, L. A., Tripathi, M., . . Simon, S. R. (2012). Physician satisfaction following electronic health record adoption in three massachusetts communities. Interact J Med Res, 1(2), e12. doi:10.2196/ijmr.2064 
Hoerbst, A., \& Schweitzer, M. (2015). A Systematic Investigation on Barriers and Critical Success Factors for Clinical Information Systems in Integrated Care Settings. Yearb Med Inform, 10(1), 79-89. doi:10.15265/IY-2015-018

Holanda, A. A., Do Carmo E Sa, H., Vieira, A. P., \& Catrib, A. M. (2012). Use and satisfaction with electronic health record by primary care physicians in a health district in Brazil. Journal of Medical Systems, 36(5), 3141-3149. doi:10.1007/s10916-011-9801-3

Holanda, A. A., do Carmo, E. Sa H. L., Vieira, A. P., \& Catrib, A. M. (2012). Use and satisfaction with electronic health record by primary care physicians in a health district in Brazil. J Med Syst, 36(5), 3141-3149. doi:10.1007/s10916-011-9801-3

Holroyd-Leduc, J. M., Lorenzetti, D., Straus, S. E., Sykes, L., \& Quan, H. (2011). The impact of the electronic medical record on structure, process, and outcomes within primary care: a systematic review of the evidence. J Am Med Inform Assoc, 18(6), 732-737. doi:10.1136/amiajnl-2010-000019

Hoonakker, P. L., Carayon, P., \& Walker, J. M. (2010). Measurement of CPOE enduser satisfaction among ICU physicians and nurses. Appl Clin Inform, 1(3), 268285. doi:10.4338/ACI-2010-03-RA-0020

Hor, C. P., O'Donnell, J. M., Murphy, A. W., O'Brien, T., \& Kropmans, T. J. (2010). General practitioners' attitudes and preparedness towards Clinical Decision Support in e-Prescribing (CDS-eP) adoption in the West of Ireland: a cross sectional study. BMC Med Inform Decis Mak, 10, 2. doi:10.1186/1472-6947-102

Houser, S. H., \& Johnson, L. A. (2008). Perceptions regarding electronic health record implementation among health information management professionals in Alabama: a statewide survey and analysis. Perspect Health Inf Manag, 5, 6.

Hsiao, C. J., Hing, E., Socey, T. C., \& Cai, B. (2011). Electronic Health Record Systems and Intent to Apply for Meaningful Use Incentives Among Officebased Physician Practices: United States, 2001-2011. NCHS Data Brief, 79.

Hsieh, S. H., Hou, I. C., Tan, C. T., Shen, P. C., Yu, H. C., Hsieh, S. L., . . Lai, F. P. (2009). Design and Implementation of Mobile Electronic Medication Administration Record. New Advances in Intelligent Decision Technologies, 199, 493-507.

Huerta, T., Thompson, M., Ford, E., \& Ford, W. (2013). Electronic health record implementation and hospitals' total factor productivity. Decision Support Systems, 55(2), 450-458. doi:http://dx.doi.org/10.1016/j.dss.2012.10.004

Ingebrigtsen, T., Georgiou, A., Clay-Williams, R., Magrabi, F., Hordern, A., Prgomet, M., . . Braithwaite, J. (2014). The impact of clinical leadership on health 
information technology adoption: systematic review. Int J Med Inform, 83(6), 393-405. doi:10.1016/j.ijmedinf.2014.02.005

IOM, Institute of Medicine. (2003). Key Capabilities of an Electronic Health Record System:Letter Report. Washington, USA: The National Academies Press.

ISO, International Organization for Standardization. (2001). Health informatics Interoperability and compatibility in messaging and communication standards - Key characteristics. Retrieved from https://www.iso.org/obp/ui/\#iso:std:33396:en

ISO, International Organization for Standardization. (2013). Usability of consumer products and products for public use - Part 2: Summative test method. Retrieved from https://www.iso.org/obp/ui/\#iso:std:iso:ts:20282:-2:ed-2:v1:en

Janamian, T., Jackson, C. L., Glasson, N., \& Nicholson, C. (2014). A systematic review of the challenges to implementation of the patient-centred medical home: lessons for Australia. Med J Aust, 201(3 Suppl), S69-73.

Jardim, S. (2013). The Electronic Health Record and its Contribution to Healthcare Information Systems Interoperability. Procedia Technology, 9(Supplement C), 940-948. doi:https://doi.org/10.1016/j.protcy.2013.12.105

Jawhari, B., Keenan, L., Zakus, D., Ludwick, D., Isaac, A., Saleh, A., \& Hayward, R. (2016). Barriers and facilitators to Electronic Medical Record (EMR) use in an urban slum. Int J Med Inform, 94, 246-254. doi:10.1016/j.ijmedinf.2016.07.015

Jennett, P., Jackson, A., Healy, T., Ho, K., Kazanjian, A., Woollard, R., . . Bates, J. (2003). A study of a rural community's readiness for telehealth. $J$ Telemed Telecare, 9(5), 259-263. doi:10.1258/135763303769211265

Jha, A., DesRoches, C., Campbell, E., Donelan, K., Rao, S., Ferris, T., . . Blumenthal, D. (2009). Use of Electronic Health Records in U.S. Hospitals. New England Journal of Medicine, 360(16), 1628-1638. doi:10.1056/NEJMsa0900592

Jha, A. K., Bates, D. W., Jenter, C., Orav, E. J., Zheng, J., Cleary, P., \& Simon, S. R. (2009). Electronic health records: use, barriers and satisfaction among physicians who care for black and Hispanic patients. Journal of Evaluation in Clinical Practice, 15(1), 158-163. doi:10.1111/j.1365-2753.2008.00975.x

Jones, E. B., \& Furukawa, M. F. (2014). Adoption and use of electronic health records among federally qualified health centers grew substantially during 2010-12. Health Aff (Millwood), 33(7), 1254-1261. doi:10.1377/hlthaff.2013.1274

Jotkowitz, A., Oh, J., Tu, C., Elkin, D., Pollack, L. A., \& Kerpen, H. (2006). The use of personal digital assistants among medical residents. Med Teach, 28(4), 382-384. doi:10.1080/01421590600607914 
Kalra, D. (2006). Electronic health record standards. Methods of Information in Medicine, 45, 136-144.

Kang, N., \& Yoon, W. (2008). Age- and experience-related user behavior differences in the use of complicated electronic devices. International Journal of HumanComputer Studies, 66(6), 425-437. doi:10.1016/j.ijhcs.2007.12.003

Kannoju, P. K., Sridhar, K. V., \& Prasad, K. S. R. (2010, 30 Nov.-2 Dec. 2010). A new paradigm of Electronic Health record for efficient implementation of healthcare delivery. Paper presented at the IEEE EMBS Conference on Biomedical Engineering and Sciences (IECBES), Kuala Lumpur, Malaysia.

Kayaalp, Mehmet. (2018). Patient Privacy in the Era of Big Data. Balkan medical journal, 35(1), 8-17. doi:10.4274/balkanmedj.2017.0966

Kemper, A. R., Uren, R. L., \& Clark, S. J. (2006). Adoption of electronic health records in primary care pediatric practices. Pediatrics, 118(1), e20-24. doi:10.1542/peds.2005-3000

Keshavjee, K., Bosomworth, J., Copen, J., Lai, J., Kucukyazici, B., Lilani, R., \& Holbrook, A. M. (2006). Best practices in EMR implementation: a systematic review. AMIA Annu Symp Proc, 982. doi:86165 [pii]

Khajouei, R., Wierenga, P. C., Hasman, A., \& Jaspers, M. W. (2011). Clinicians satisfaction with CPOE ease of use and effect on clinicians' workflow, efficiency and medication safety. Int J Med Inform, 80(5), 297-309. doi:S13865056(11)00055-4 [pii]

10.1016/j.ijmedinf.2011.02.009

Khalifa, M., \& Alswailem, O. (2015). Hospital Information Systems (HIS) Acceptance and Satisfaction: A Case Study of a Tertiary Care Hospital. Procedia Computer Science, 63(Supplement C), 198-204. doi:https://doi.org/10.1016/j.procs.2015.08.334

Khatun, F., Heywood, A. E., Ray, P. K., Hanifi, S. M., Bhuiya, A., \& Liaw, S. T. (2015). Determinants of readiness to adopt mHealth in a rural community of Bangladesh. Int J Med Inform, 84(10), 847-856. doi:10.1016/j.ijmedinf.2015.06.008

Khudair, A. (2008, 21-22 May 2008). Electronic health records: Saudi physicians' perspective. Paper presented at the 2008 5th IET Seminar on Appropriate Healthcare Technologies for Developing Countries, London, UK.

Kim, S., Lee, K., Hwang, H., \& Yoo, S. (2016). Analysis of the factors influencing healthcare professionals' adoption of mobile electronic medical record (EMR) using the unified theory of acceptance and use of technology (UTAUT) in a 
tertiary hospital. BMC Medical Informatics and Decision Making, 16, 12. doi:10.1186/s 12911-016-0249-8

Koivunen, M., Hatonen, H., \& Valimaki, M. (2008). Barriers and facilitators influencing the implementation of an interactive Internet-portal application for patient education in psychiatric hospitals. Patient Education and Counseling, 70(3), 412-419. doi:10.1016/j.pec.2007.11.002

Kruse, C. S., DeShazo, J. P., Kim, F., \& Fulton, L. (2014). Factors Associated With Adoption of Health Information Technology: A Conceptual Model Based on a Systematic Review. JMIR Medical Informatics, 2(1), e9. doi:10.2196/medinform.3106

Kruse, C. S., Kothman, K., Anerobi, K., \& Abanaka, L. (2016). Adoption Factors of the Electronic Health Record: A Systematic Review. JMIR Med Inform, 4(2), e19. doi:10.2196/medinform.5525

Kruse, C. S., Kristof, C., Jones, B., Mitchell, E., \& Martinez, A. (2016). Barriers to Electronic Health Record Adoption: a Systematic Literature Review. J Med Syst, 40(12), 252. doi:10.1007/s10916-016-0628-9

Kruse, C. S., Mileski, M., Alaytsev, V., Carol, E., \& Williams, A. S. (2015). Adoption factors associated with electronic health record among long-term care facilities: a systematic review. BMJ Open, 5(1). doi:10.1136/bmjopen-2014-006615

Kuo, K. M., Liu, C. F., \& Ma, C. C. (2013). An investigation of the effect of nurses' technology readiness on the acceptance of mobile electronic medical record systems. BMC Med Inform Decis Mak, 13, 88. doi:10.1186/1472-6947-13-88

Kysh, L. (2013). Difference between a systematic review and a literature review. Retrieved from https://figshare.com/articles/Difference_between_a_systematic_review_and_a_li terature review/766364

La Torre, G., Backhaus, I., \& Mannocci, A. (2015). Rating for narrative reviews: concept and development of the International Narrative Systematic Assessment tool. Senses and Sciences, 2(2).

Laerum, H., Karlsen, T. H., \& Faxvaag, A. (2004). Use of and attitudes to a hospital information system by medical secretaries, nurses and physicians deprived of the paper-based medical record: a case report. BMC Med Inform Decis Mak, 4, 18. doi:10.1186/1472-6947-4-18

Lai, T. Y., Leung, G. M., Wong, I. O., \& Johnston, J. M. (2004). Do doctors act on their self-reported intention to computerize? A follow-up population-based survey in Hong Kong. Int J Med Inform, 73(5), 415-431.

doi:10.1016/j.ijmedinf.2004.03.004 


\section{S1386505604000656 [pii]}

Laramee, A. S., Bosek, M., Shaner-McRae, H., \& Powers-Phaneuf, T. (2012). A comparison of nurse attitudes before implementation and 6 and 18 months after implementation of an electronic health record. Comput Inform Nurs, 30(10), 521-530. doi:10.1097/NXN.0b013e3182573b04

Lee, F., Teich, J. M., Spurr, C. D., \& Bates, D. W. (1996). Implementation of physician order entry: user satisfaction and self-reported usage patterns. J Am Med Inform Assoc, 3(1), 42-55.

Lennon, M. R., Bouamrane, M. M., Devlin, A. M., O'Connor, S., O'Donnell, C., Chetty, U., . . Mair, F. S. (2017). Readiness for Delivering Digital Health at Scale: Lessons From a Longitudinal Qualitative Evaluation of a National Digital Health Innovation Program in the United Kingdom. J Med Internet Res, 19(2), e42. doi:10.2196/jmir.6900

Leon, S. A., Fontelo, P., Green, L., Ackerman, M., \& Liu, F. (2007). Evidence-based medicine among internal medicine residents in a community hospital program using smart phones. BMC Med Inform Decis Mak, 7, 5. doi:10.1186/1472-6947$7-5$

Levy, P.S., \& Lemeshow, S. (2013). Sampling of Populations: Methods and Applications (4th ed. Vol. 1). Albany, NY, USA: Wiley.

Littlewood, J., \& Yousuf, S. (2000). Primary health care in Saudi Arabia: applying global aspects of health for all, locally. Journal of Advanced Nursing, 32(3), 675-681. doi:DOI 10.1046/j.1365-2648.2000.01527.x

Loomis, G. A., Ries, J. S., Saywell, R. M., Jr., \& Thakker, N. R. (2002). If electronic medical records are so great, why aren't family physicians using them? J Fam Pract, 51(7), 636-641.

Lorenzi, N. M., Kouroubali, A., Detmer, D. E., \& Bloomrosen, M. (2009). How to successfully select and implement electronic health records (EHR) in small ambulatory practice settings. BMC Med Inform Decis Mak, 9, 15. doi:10.1186/1472-6947-9-15

Lorenzi, N. M., Smith, J. B., Conner, S. R., \& Campion, T. R. (2004). The Success Factor Profile for clinical computer innovation. Stud Health Technol Inform, 107(Pt 2), 1077-1080.

Ludwick, D. a, \& Doucette, John. (2009a). Primary Care Physicians' Experience with Electronic Medical Records: Barriers to Implementation in a Fee-for-Service Environment. International Journal of Telemedicine and Applications, 2009, 853524-853524. doi:10.1155/2009/853524 
Ludwick, D. A., \& Doucette, J. (2009b). Adopting electronic medical records in primary care: lessons learned from health information systems implementation experience in seven countries. Int J Med Inform, 78(1), 22-31.

doi:10.1016/j.ijmedinf.2008.06.005

Luna, D., Almerares, A., Mayan, J. C., 3rd, Gonzalez Bernaldo de Quiros, F., \& Otero, C. (2014). Health Informatics in Developing Countries: Going beyond Pilot Practices to Sustainable Implementations: A Review of the Current Challenges. Healthc Inform Res, 20(1), 3-10. doi:10.4258/hir.2014.20.1.3

Madore, A., Rosenberg, J., Muyindike, W. R., Bangsberg, D. R., Bwana, M. B., Martin, J. N., . . Weintraub, R. (2015). Implementation of electronic medical records requires more than new software: Lessons on integrating and managing health technologies from Mbarara, Uganda. Healthc (Amst), 3(4), 264-269. doi:10.1016/j.hjdsi.2015.08.006

Mair, F. S., May, C., O’Donnell, C., Finch, T., Sullivan, F., \& Murray, E. (2012). Factors that promote or inhibit the implementation of e-health systems: an explanatory systematic review. Bull World Health Organ, 90. doi:10.2471/blt.11.099424

Makam, A. N., Lanham, H. J., Batchelor, K., Samal, L., Moran, B., Howell-Stampley, T., . . Halm, E. A. (2013). Use and satisfaction with key functions of a common commercial electronic health record: a survey of primary care providers. BMC Med Inform Decis Mak, 13, 86. doi:10.1186/1472-6947-13-86

Maley, C.H. (2012). Project Management Concepts, Methods, and Techniques (1st ed.). United Kingdom CRC Press.

Martikainen, S., Viitanen, J., Korpela, M., \& Laaveri, T. (2012). Physicians' experiences of participation in healthcare IT development in Finland: willing but not able. Int J Med Inform, 81(2), 98-113. doi:10.1016/j.ijmedinf.2011.08.014

McAlearney, A. S., Hefner, J. L., Sieck, C., Rizer, M., \& Huerta, T. R. (2014). Evidence-based management of ambulatory electronic health record system implementation: an assessment of conceptual support and qualitative evidence. Int J Med Inform, 83(7), 484-494. doi:10.1016/j.ijmedinf.2014.04.002

McAlearney, A. S., Schweikhart, S. B., \& Medow, M. A. (2005). Organizational and physician perspectives about facilitating handheld computer use in clinical practice: results of a cross-site qualitative study. J Am Med Inform Assoc, 12(5), 568-575. doi:10.1197/jamia.M1816

McAlearney, A. S., Sieck, C., Hefner, J., Robbins, J., \& Huerta, T. R. (2013). Facilitating ambulatory electronic health record system implementation: evidence from a qualitative study. Biomed Res Int, 2013, 629574. doi:10.1155/2013/629574 
Mekhjian, H. S., Kumar, R. R., Kuehn, L., Bentley, T. D., Teater, P., Thomas, A., . . . Ahmad, A. (2002). Immediate benefits realized following implementation of physician order entry at an academic medical center. J Am Med Inform Assoc, 9(5), 529-539.

Menachemi, N., \& Collum, T. H. (2011). Benefits and drawbacks of electronic health record systems. Risk Manag Healthc Policy, 4, 47-55.

doi:10.2147/RMHP.S12985

Middleton, B., Bloomrosen, M., Dente, M. A., Hashmat, B., Koppel, R., Overhage, J. M., ... Zhang, J. (2013). Enhancing patient safety and quality of care by improving the usability of electronic health record systems: recommendations from AMIA. Journal of the American Medical Informatics Association, 20(e1), e2-e8. doi:10.1136/amiajnl-2012-001458

Middleton, B., Hammond, W. E., Brennan, P. F., \& Cooper, G. F. (2005). Accelerating U.S. EHR adoption: how to get there from here. recommendations based on the 2004 ACMI retreat. J Am Med Inform Assoc, 12(1), 13-19. doi:10.1197/jamia.M1669

Miles, C. . (2013). The Project Champion: A Management Best Practice. Retrieved from https://smallbiztrends.com/2013/12/what-is-a-project-champion.html

Miles, J., \& Gilbert, P. (2005). A handbook of research methods for clinical and health psychology. United Kingdom Oxford University Press.

Miller, R. H., West, C., Brown, T. M., Sim, I., \& Ganchoff, C. (2005). The value of electronic health records in solo or small group practices. Health Aff (Millwood), 24(5), 1127-1137. doi:10.1377/hlthaff.24.5.1127

Mitchell, M., \& Jolley, J. (2009). Research Design Explained (8th ed.). Pennsylvania, USA: Cengage Learning.

MoH, Ministry of Health. (2013a). National e-Health Strategy: The Roadmap of Projects. Retrieved from http://www.moh.gov.sa/en/Ministry/nehs/Pages/TheRoadmap-of-Projects.aspx

MoH, Ministry of Health. (2011a). The New Primary Healthcare Center Systems. Retrieved from http://www.moh.gov.sa/en/Ministry/nehs/Pages/The-New-PHCSystems.aspx

MoH, Ministry of Health. (2011b). The Statistics Book for the year 1432H. Retrieved from http://www.moh.gov.sa/Ministry/Statistics/book/flash/1432/MOH_Report_1432. $\underline{\mathrm{html}}$ 
MoH, Ministry of Health. (2012). The Statistics Book for the year 1433H. Retrieved from https://www.moh.gov.sa/Ministry/Statistics/book/Documents/1433.pdf

MoH, Ministry of Health. (2013b). National E-health stategy. Retrieved from http://www.moh.gov.sa/en/Ministry/nehs/Pages/Ehealth.aspx

MoH, Ministry of Health. (2013c). The New Primary Healthcare Center Systems. Retrieved from http://www.moh.gov.sa/en/Ministry/nehs/Pages/The-New-PHCSystems.aspx

Moher, D., Liberati, A., Tetzlaff, J., \& Altman, D. G. (2009). Preferred reporting items for systematic reviews and meta-analyses: the PRISMA statement. Ann Intern Med, 151(4), 264-269, W264.

Moody, L. E., Slocumb, E., Berg, B., \& Jackson, D. (2004). Electronic health records documentation in nursing: nurses' perceptions, attitudes, and preferences. Comput Inform Nurs, 22(6), 337-344.

Morton, M. E., \& Wiedenbeck, S. (2010). EHR acceptance factors in ambulatory care: a survey of physician perceptions. Perspect Health Inf Manag, 7, 1c.

Murray, E., May, C., \& Mair, F. (2010). Development and formative evaluation of the e-Health Implementation Toolkit (e-HIT). BMC Medical Informatics and Decision Making, 10, 61-61. doi:10.1186/1472-6947-10-61

Nakamura, M. M., Ferris, T. G., DesRoches, C. M., \& Jha, A. K. (2010). Electronic health record adoption by children's hospitals in the United States. Arch Pediatr Adolesc Med, 164(12), 1145-1151. doi:10.1001/archpediatrics.2010.234

Negash, S., Musa, P., Vogel, D., \& Sahay, S. (2018). Healthcare information technology for development: improvements in people's lives through innovations in the uses of technologies. Information Technology for Development, 24(2), 189-197. doi:10.1080/02681102.2018.1422477

Nguyen, L., Bellucci, E., \& Nguyen, L. T. (2014). Electronic health records implementation: an evaluation of information system impact and contingency factors. Int J Med Inform, 83(11), 779-796. doi:10.1016/j.ijmedinf.2014.06.011

Niazkhani, Z., van der Sijs, H., Pirnejad, H., Redekop, W. K., \& Aarts, J. (2009). Same system, different outcomes: comparing the transitions from two paper-based systems to the same computerized physician order entry system. Int J Med Inform, 78(3), 170-181. doi:S1386-5056(08)00106-8 [pii]

10.1016/j.ijmedinf.2008.06.012

O'Connor, E. J., \& Fiol, C. M. (2006). Creating readiness and involvement. Physician Exec, 32(1), 72-74. 
O'Meara, E. (2007). The effects of electronic documentation in the ambulatory surgery setting. AORN J, 86(6), 970-979.

Offredy, M., \& Vickers, P. (2010). Developing a Healthcare Research Proposal: An Interactive Student Guide (1st ed.). Hertfordshire, United Kingdom John Wiley \& Sons.

Ortiz, E., Clancy, C. M., \& Ahrq. (2003). Use of information technology to improve the quality of health care in the United States. Health Serv Res, 38(2), xi-xxii.

Ovretveit, J., Scott, T., Rundall, T. G., Shortell, S. M., \& Brommels, M. (2007). Improving quality through effective implementation of information technology in healthcare. Int J Qual Health Care, 19. doi:10.1093/intqhe/mzm031

Pantaleoni, J. L., Stevens, L. A., Mailes, E. S., Goad, B. A., \& Longhurst, C. A. (2015). Successful Physician Training Program for Large Scale EMR Implementation. Applied Clinical Informatics, 6(1), 80-95. doi:10.4338/ACI-2014-09-CR-0076

Pare, G., Sicotte, C., Jaana, M., \& Girouard, D. (2008). Prioritizing the risk factors influencing the success of clinical information system projects. A Delphi study in Canada. Methods Inf Med, 47(3), 251-259.

Pare, G., Sicotte, C., Poba-Nzaou, P., \& Balouzakis, G. (2011). Clinicians' perceptions of organizational readiness for change in the context of clinical information system projects: insights from two cross-sectional surveys. Implement Sci, 6, 15. doi:10.1186/1748-5908-6-15

Perera, F., \& Peiro, M. (2012). Strategic Planning in Healthcare Organizations. Revista Española de Cardiología (English Edition), 65(08), 749-754.

Piliouras, T., Pui Lam, Y., Housheng, H., Xin, L., Siddaramaiah, V., \& Sultana, N. (2011, 6-6 May 2011). Selection of electronic health records software: Challenges, considerations, and recommendations. Paper presented at the Systems, Applications and Technology Conference (LISAT), 2011 IEEE Long Island.

Pitcher, E. (2010). CNO Role in the Implementation of an Electronic Health Record. Nurse Leader, 8(3), 32-35. doi:http://dx.doi.org/10.1016/j.mnl.2010.03.005

Podichetty, V., \& Penn, D. (2004). The progressive roles of electronic medicine: benefits, concerns, and costs. Am J Med Sci, 328(2), 94-99.

Polit, D.F., Beck, C.T., \& Hungler, B.P. (2002). Essentials of nursing research: methods, appraisal, and utilization. USA: Lippincott Williams \& Wilkins. 
Poon, E. G., Blumenthal, D., Jaggi, T., Honour, M. M., Bates, D. W., \& Kaushal, R. (2004). Overcoming barriers to adopting and implementing computerized physician order entry systems in US hospitals. Health Aff, 23. doi:10.1377/hlthaff.23.4.184

Qurban, M. H., \& Austria, R. D. (2008). Public Perception on E-health Services: Implications of Prelimary Findings of KFMMC for Military Hospitals in KSA. Paper presented at the European and Mediterranean Conference on Information Systems, Dubai. http://www.iseing.org/emcis/emcis2008/Proceedings/Refereed\%20Papers/Contri butions/C\%209/emcis2008_Qurban_and_Austria_final_revision.pdf

Ramanathan, T.R. (2009). The Role of Organisational Change Management in Offshore Outsourcing of Information Technology Services: Qualitative Case Studies from a Multinational Pharmaceutical Company. USA: Universal Publishers.

Rantz, M. J., Alexander, G., Galambos, C., Flesner, M. K., Vogelsmeier, A., Hicks, L., . . . Greenwald, L. (2011). The use of bedside electronic medical record to improve quality of care in nursing facilities: a qualitative analysis. Comput Inform Nurs, 29(3), 149-156. doi:10.1097/NCN.0b013e3181f9db79

Rao, S., DesRoches, C., Donelan, K., Campbell, E., Miralles, P., \& Jha, A. (2011). Electronic health records in small physician practices: availability, use, and perceived benefits. J Am Med Inform Assoc, 18(3), 271-275. doi:10.1136/amiajnl-2010-000010

Redd, T., Read-Brown, S., Choi, D., Yackel, T., Tu, D., \& Chiang, M. (2014). Electronic health record impact on productivity and efficiency in an academic pediatric ophthalmology practice. Journal of American Association for Pediatric Ophthalmology and Strabismus, 18(6), 584-589. doi:http://dx.doi.org/10.1016/j.jaapos.2014.08.002

Reja, U., Manfreda, K., Hlebec, V., \& Vehovar, V. (2003). Open-ended vs. Close-ended Questions in Web Questionnaires. Metodološki zvezki, 19.

Rezaeibagha, F., Win, K. T., \& Susilo, W. (2015). A systematic literature review on security and privacy of electronic health record systems: technical perspectives. HIM J, 44(3), 23-38.

Rizer, M. K., Kaufman, B., Sieck, C. J., Hefner, J. L., \& McAlearney, A. S. (2015). Top 10 Lessons Learned from Electronic Medical Record Implementation in a Large Academic Medical Center. Perspect Health Inf Manag, 12, 1g.

Robertson, J. (2013). Where Doctors Go Digital: Top 10 Countries. Retrieved from https://www.bloomberg.com/news/articles/2013-06-27/where-doctors-godigital-top-10-countries 
Robson, C. (2011). Real World Research (3rd ed.). United Kingdom John Wiley \& Sons.

Ross, J., Stevenson, F., Lau, R., \& Murray, E. (2016). Factors that influence the implementation of e-health: a systematic review of systematic reviews (an update). Implement Sci, 11(1), 146. doi:10.1186/s13012-016-0510-7

Ross, S. E., Schilling, L. M., Fernald, D. H., Davidson, A. J., \& West, D. R. (2010). Health information exchange in small-to-medium sized family medicine practices: motivators, barriers, and potential facilitators of adoption. Int J Med Inform, 79(2), 123-129. doi:10.1016/j.ijmedinf.2009.12.001

Saad, A. (2004). Factors Influencing the Utilisation of Public and Private Primary Health Care Services in Riyadh City. Journal of King Abdulaziz University, 19(1), 3-27.

Safdari, R., Ghazisaeidi, M., \& Jebraeily, M. (2015). Electronic Health Records: Critical Success Factors in Implementation. Acta Informatica Medica, 23(2), 102-104. doi:10.5455/aim.2015.23.102-104

Sahay, S. (2001). Special Issue on "IT and Health Care in Developing Countries". The Electronic Journal of Information Systems in Developing Countries, 5(1), 1-6. doi:10.1002/j.1681-4835.2001.tb00029.x

Saleh, S., Khodor, R., Alameddine, M., \& Baroud, M. (2016). Readiness of healthcare providers for eHealth: the case from primary healthcare centers in Lebanon. BMC Health Services Research, 16, 644. doi:10.1186/s12913-016-1896-2

Samoutis, G., Soteriades, E. S., Kounalakis, D. K., Zachariadou, T., Philalithis, A., \& Lionis, C. (2007). Implementation of an electronic medical record system in previously computer-naive primary care centres: a pilot study from Cyprus. Inform Prim Care, 15(4), 207-216.

Sanchez, L., Savin, S., \& Vasileva, V. (2005, 18/09/2013). Key success factors in implementing electronic medical records in university hospital of Rennes. Paper presented at the L'Ecole Nationale de la Santé Publique (National School of Public Health), Rennes, Rennes, France.

Scott, J., Rundall, T., Vogt, T., \& Hsu, J. (2005). Kaiser Permanente's experience of implementing an electronic medical record: a qualitative study. BMJ (Clinical research ed.), 331(7528), 1313-1316. doi:10.1136/bmj.38638.497477.68

Secginli, S., Erdogan, S., \& Monsen, K. A. (2014). Attitudes of health professionals towards electronic health records in primary health care settings: a questionnaire survey. Inform Health Soc Care, 39(1), 15-32.

doi:10.3109/17538157.2013.834342 
Shortliffe, E.H., Perreault, L.E., Wiederhold, G., \& Fagan, L.M. (1990). Medical informatics: computer applications in health care. Boston, USA: AddisonWesley Pub. Co.

Sicotte, C., \& Pare, G. (2010). Success in health information exchange projects: solving the implementation puzzle. Soc Sci Med, 70(8), 1159-1165.

doi:10.1016/j.socscimed.2009.11.041

Silverman, D. (2013). Doing Qualitative Research: A Practical Handbook. Australia SAGE Publications.

Sim, I., Gorman, P., Greenes, R. A., Haynes, R. B., Kaplan, B., Lehmann, H., \& Tang, P. C. (2001). Clinical decision support systems for the practice of evidencebased medicine. J Am Med Inform Assoc, 8(6), 527-534.

Simba, D. O., \& Mwangu, M. (2004). Application of ICT in strengthening health information systems in developing countries in the wake of globalisation. Afr Health Sci, 4(3), 194-198.

Simon, S. R., Kaushal, R., Cleary, P. D., Jenter, C. A., Volk, L. A., Poon, E. G., . . Bates, D. W. (2007). Correlates of electronic health record adoption in office practices: a statewide survey. J Am Med Inform Assoc, 14(1), 110-117. doi:10.1197/jamia.M2187

Simon, S. R., Kaushal, R., Jenter, C. A., Volk, L. A., Burdick, E., Poon, E. G., . . . Bates, D. W. (2008). Readiness for electronic health records: comparison of characteristics of practices in a collaborative with the remainder of Massachusetts. Inform Prim Care, 16(2), 129-137.

Simon, S. R., Keohane, C. A., Amato, M., Coffey, M., Cadet, B., Zimlichman, E., \& Bates, D. W. (2013). Lessons learned from implementation of computerized provider order entry in 5 community hospitals: a qualitative study. BMC Med Inform Decis Mak, 13, 67. doi:10.1186/1472-6947-13-67

Singh, R., Lichter, M. I., Danzo, A., Taylor, J., \& Rosenthal, T. (2012). The adoption and use of health information technology in rural areas: results of a national survey. J Rural Health, 28(1), 16-27. doi:10.1111/j.1748-0361.2011.00370.x

Sittig, D. F., Krall, M., Kaalaas-Sittig, J., \& Ash, J. S. (2005). Emotional aspects of computer-based provider order entry: a qualitative study. J Am Med Inform Assoc, 12(5), 561-567. doi:10.1197/jamia.M1711

Slight, S. P., Quinn, C., Avery, A. J., Bates, D. W., \& Sheikh, A. (2014). A qualitative study identifying the cost categories associated with electronic health record implementation in the UK. J Am Med Inform Assoc, 21(e2), e226-231. doi:10.1136/amiajnl-2013-002404 
Smith, K., Smith, V., Krugman, M., \& Oman, K. (2005). Evaluating the impact of computerized clinical documentation. Comput Inform Nurs, 23(3), 132-138.

Smith, P. D. (2003). Implementing an EMR system: one clinic's experience. Fam Pract Manag, 10(5), 37-42.

Snyder-Halpern, R. (2002). Development and pilot testing of an Organizational Information Technology/Systems Innovation Readiness Scale (OITIRS). Proc AMIA Symp, 702-706.

Snyder, R. A., \& Fields, W. L. (2006). Measuring hospital readiness for information technology (IT) innovation: A multisite study of the Organizational Information Technology Innovation Readiness Scale. J Nurs Meas, 14(1), 45-55.

Sprague, L. (2004). Electronic Health Records: How Close? How Far to Go? Retrieved from http://www.nhpf.org/library/issue-briefs/IB800_EHRs.pdf

Stansfield, S. K., Walsh, J., Prata, N., \& Evans, T. (2006). Information to Improve Decision Making for Health. In Disease Control Priorities in Developing Countries (2nd ed.). Washington (DC).

Starfield, B. (1998). Primary Care: Balancing Health Needs, Services, and Technology. United Kingdom OXFORD University Press.

Starfield, B., Shi, L., \& Macinko, J. (2005). Contribution of primary care to health systems and health. Milbank $Q, 83(3), 457-502$. doi:10.1111/j.14680009.2005.00409.x

Steininger, K. , Stiglbauer, B., Baumgartner, B., \& Engleder, B. (2014). Factors Explaining Physicians' Acceptance of Electronic Health Records. Paper presented at the 47th Hawaii International Conference on System Science, USA.

Sterns, A. A. (2005). Curriculum design and program to train older adults to use personal digital assistants. Gerontologist, 45(6), 828-834. doi:45/6/828 [pii]

Stone, C. (2014). A Glimpse at EHR Implementation Around the World: The Lessons the US Can Learn. Health Institute for E-Health Policy, May. The Health Institute for E-Health Policy. USA. Retrieved from http://www.ehealthpolicy.org/docs/A_Glimpse_at_EHR_Implementation_Around the_World 1_ChrisStone.pdf

Sturzlinger, H., Hiebinger, C., Pertl, D., \& Traurig, P. (2009). Computerized Physician Order Entry - effectiveness and efficiency of electronic medication ordering with decision support systems. GMS Health Technol Assess, 5, Doc07. doi:10.3205/hta000069 
SU, Swansea University. (2019). Systematic Reviews: Where to search. Retrieved from http://libguides.swansea.ac.uk/sysreviews/wheretosearch

Tan, J. (2005). E-Health Care Information Systems: An Introduction for Students and Professionals (1st ed.). USA: Jossey-Bass.

Taylor, H. (2005). Health Information Privacy (HIPAA) Notices Have Improved Public's Confidence That Their Medical information Is Being Handled Properly.

Techopedia. (2017). Project Planning. Retrieved from https://www.techopedia.com/definition/14005/project-planning

Terry, A. L., Giles, G., Brown, J. B., Thind, A., \& Stewart, M. (2009). Adoption of electronic medical records in family practice: the providers' perspective. Family Medicine, 41(7), 508-512.

Terry, A., Thorpe, C., Giles, G., Brown, J. B., Harris, S., Reid, G., . . . Stewart, M. (2008). Implementing electronic health records: Key factors in primary care. Canadian Family Physician, 54(5), 730-736.

Thakkar, M., \& Davis, D. C. (2006). Risks, barriers, and benefits of EHR systems: a comparative study based on size of hospital. Perspect Health Inf Manag, 3, 5.

Thompson, S.K. (2012). Sampling. USA: Wiley.

Tongco, M. (2007). Purposive sampling as a tool for informant selection. Ethnobotany Research and applications, 5, 147-158.

Tsiknakis, M., \& Kouroubali, A. (2009). Organizational factors affecting successful adoption of innovative eHealth services: a case study employing the FITT framework. Int J Med Inform, 78(1), 39-52. doi:10.1016/j.ijmedinf.2008.07.001

Tumulty, G. (2001). Professional development of nursing in Saudi Arabia. J Nurs Scholarsh, 33(3), 285-290.

Unger, M. D., Aldrich, A. M., Hefner, J. L., \& Rizer, M. K. (2014). A journey through meaningful use at a large academic medical center: lessons of leadership, administration, and technical implementation. Perspect Health Inf Manag, 11, 1f.

Urban, E., Ose, D., Joos, S., Szecsenyi, J., \& Miksch, A. (2012). Technical support and delegation to practice staff - status quo and (possible) future perspectives for primary health care in Germany. BMC Med Inform Decis Mak, 12, 81. doi:10.1186/1472-6947-12-81 
Walji, M. F., Taylor, D., Langabeer, J., \& Valenza, J. A. (2009). Factors influencing implementation and outcomes of a dental electronic patient record system. Journal of Dental Education, 73(5), 589-600.

Walker, J., Bieber, E., \& Richards, F. (2006). Implementing an electronic health record system. New York; London: Springer.

Wang, P. S., Lane, M., Olfson, M., Pincus, H. A., Wells, K. B., \& Kessler, R. C. (2005). Twelve-month use of mental health services in the United States: results from the National Comorbidity Survey Replication. Arch Gen Psychiatry, 62(6), 629640. doi:10.1001/archpsyc.62.6.629

Wang, T., \& Biedermann, S. (2012). Adoption and utilization of electronic health record systems by long-term care facilities in Texas. Perspect Health Inf Manag, $9,1 \mathrm{~g}$.

Weiner, B. (2009). A theory of organizational readiness for change. Implementation Science, 4(1), 67. doi:10.1186/1748-5908-4-67

Weiner, M., Gress, T., Thiemann, D. R., Jenckes, M., Reel, S. L., Mandell, S. F., \& Bass, E. B. (1999). Contrasting views of physicians and nurses about an inpatient computer-based provider order-entry system. J Am Med Inform Assoc, 6(3), 234-244.

Weiskopf, N., \& Weng, C. (2013). Methods and dimensions of electronic health record data quality assessment: enabling reuse for clinical research. J Am Med Inform Assoc, 20(1), 144-151. doi:10.1136/amiajnl-2011-000681

Were, M. C., \& Meslin, E. M. (2011). Ethics of implementing Electronic Health Records in developing countries: points to consider. AMIA Annu Symp Proc, 2011, 1499-1505.

Whitacre, B. E., \& Williams, R. S. (2015). Electronic medical record adoption in Oklahoma practices: rural-urban differences and the role of broadband availability. J Rural Health, 31(1), 47-57. doi:10.1111/jrh.12086

WHO, World Health Organization. (2007). Electronic Health Records: Manual for Developing Countries. Retrieved 10/12/2014, from WHO Regional Office for the Western https://apps.who.int/iris/bitstream/handle/10665/207504/9290612177_eng.pdf?s equence $=1 \&$ isAllowed $=y$

WHO, World Health Organization. (2013a). Country Cooperation Strategy for WHO and Saudi Arabia 2012-2016 Retrieved from Saudi Arabia http://apps.who.int/iris/bitstream/10665/113227/1/CCS_Saudia_2013_EN_1491 $\underline{4 . p d f}$ 
WHO, World Health Organization. (2013b). Health Systems Strengthening Glossary. Retrieved from https://www.who.int/healthsystems/Glossary_January2011.pdf?ua=1

Wolf, L., Harvell, J., \& Jha, A. K. (2012). Hospitals ineligible for federal meaningfuluse incentives have dismally low rates of adoption of electronic health records. Health Aff (Millwood), 31(3), 505-513. doi:10.1377/hlthaff.2011.0351

Xierali, I. M., Hsiao, C. J., Puffer, J. C., Green, L. A., Rinaldo, J. C., Bazemore, A. W., . . . Phillips, R. L., Jr. (2013). The rise of electronic health record adoption among family physicians. Ann Fam Med, 11(1), 14-19. doi:10.1370/afm.1461

Yontz, L. S., Zinn, J. L., \& Schumacher, E. J. (2015). Perioperative nurses' attitudes toward the electronic health record. J Perianesth Nurs, 30(1), 23-32. doi:10.1016/j.jopan.2014.01.007

Yoon-Flannery, K., Zandieh, S. O., Kuperman, G. J., Langsam, D. J., Hyman, D., \& Kaushal, R. (2008). A qualitative analysis of an electronic health record (EHR) implementation in an academic ambulatory setting. Inform Prim Care, 16(4), 277-284.

Yusif, S., Hafeez-Baig, A., \& Soar, J. (2017). e-Health readiness assessment factors and measuring tools: A systematic review. Int J Med Inform, 107, 56-64. doi:https://doi.org/10.1016/j.ijmedinf.2017.08.006

Yusof, M. M., \& Aziz, K. A. (2015). Evaluation of Organizational Readiness in Information Systems Adoption: A Case Study. Asia-Pacific Journal of Information Technology and Multimedia, 4(2).

Yusof, M. M., Kuljis, J., Papazafeiropoulou, A., \& Stergioulas, L. K. (2008). An evaluation framework for Health Information Systems: human, organization and technology-fit factors (HOT-fit). Int J Med Inform, 77(6), 386-398. doi:10.1016/j.ijmedinf.2007.08.011

Zahabi, M., David, B., \& Manida, S. (2015). Usability and Safety in Electronic Medical Records Interface Design:A Review of Recent Literature and Guideline Formulation. Hum Factors, 57(5), 805-834. doi:10.1177/0018720815576827

Zandieh, S. O., Yoon-Flannery, K., Kuperman, G. J., Hyman, D., \& Kaushal, R. (2008). Correlates of expected satisfaction with electronic health records in office practices by practitioners. AMIA Annu Symp Proc, 1190.

Zheng, K., Padman, R., Krackhardt, D., Johnson, M., \& Diamond, H. (2010). Social networks and physician adoption of electronic health records: insights from an empirical study. J Am Med Inform Assoc, 17(3), 328-336.

doi:10.1136/jamia.2009.000877 
Ziefle, M., \& Bay, S. (2005). How older adults meet complexity: Aging effects on the usability of different mobile phones. Behaviour \& Information Technology, 24(5), 375-389. doi:citeulike-article-id:369806

\section{Appendices A}




\title{
11.1 Ethical approval from Swansea University
}

Swansea University

Prifysgol Abertawe

March $29^{\text {th }}, 2017$

\begin{abstract}
Haitham Alzghhaibi
Postgraduate student
\end{abstract}

Swansea University

Dear Haitham,

RE: "IMPLEMENTATION OF THE ELECTRONIC HEALTH RECORD SYSTEMS IN THE PRIMARY HEALTHCARE CENTRES IN SAUDI ARABIA".

I would like to confirm that ethical approval was given for the above PhD study on April $11^{\text {th }}$ 2014.

Yours sincerely

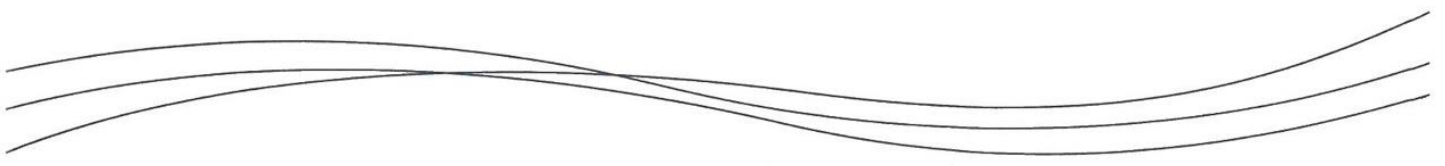

Yr Athro Ceri Phillips, Pennaeth Y Coleg • Professor Ceri Phillips, Head of College

Y Gofrestra Academaidd, Parc Singleton, Abertawe SA2 8PP, DU • College of Human and Health Sciences, Singleton Park, Swansea SA2 8PP, UK Ffôn/Tel: +44 (0)1792 295789 Ffacs/Fax: +44 (0)1792 295487 Gwefan/Website: www.swansea ac.uk/humanandhealthsciences

Mae Pritysgol Abertawe yn elusen gofrestredig. Rif.1138342 | Swansea University is a registered charity. No. 1138342

Mae'r Britysgol yn croesawu gohebiaeth yn Gymraeg ac yn Saesneg I The University welcomes correspondence in Welsh and English 


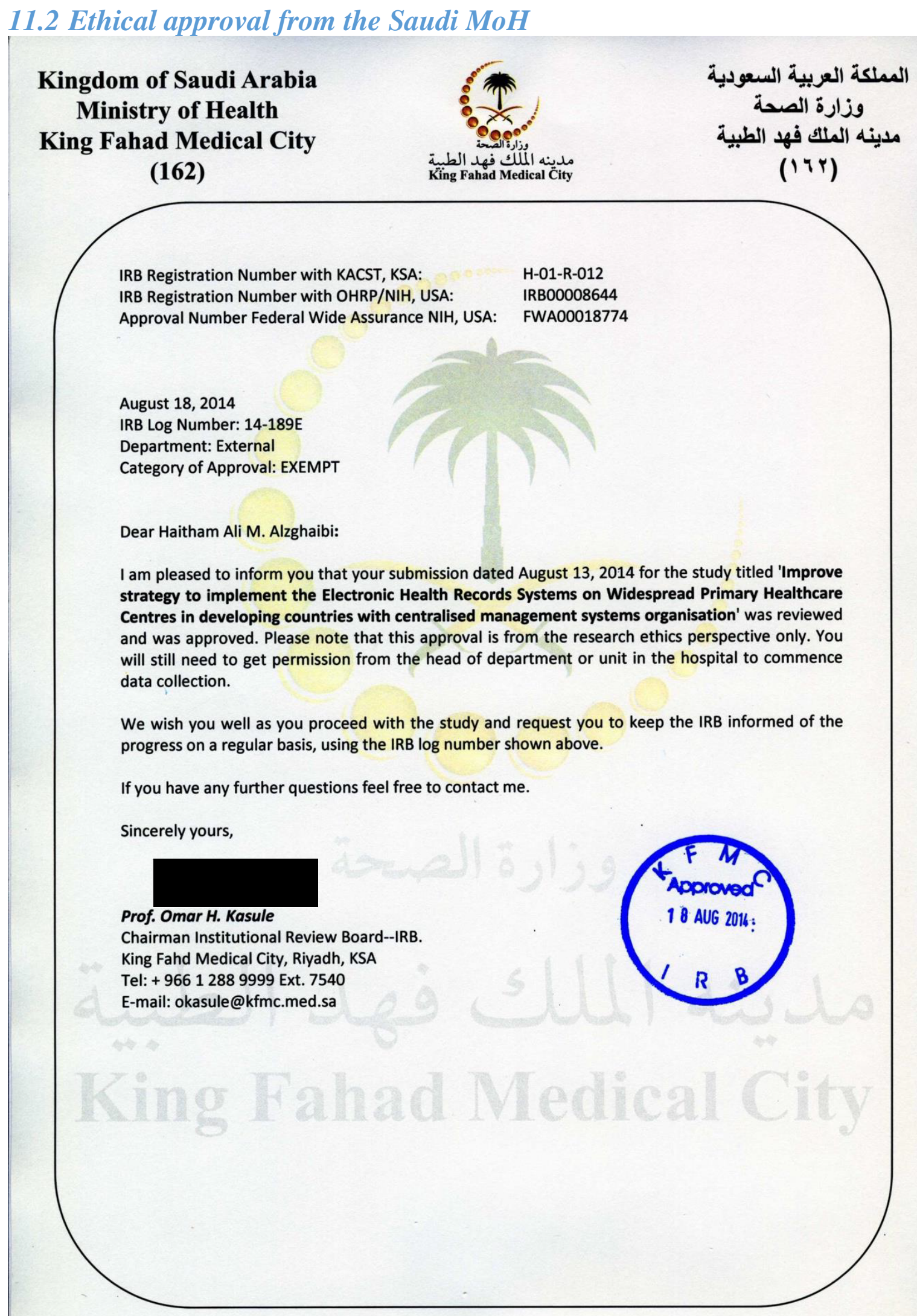




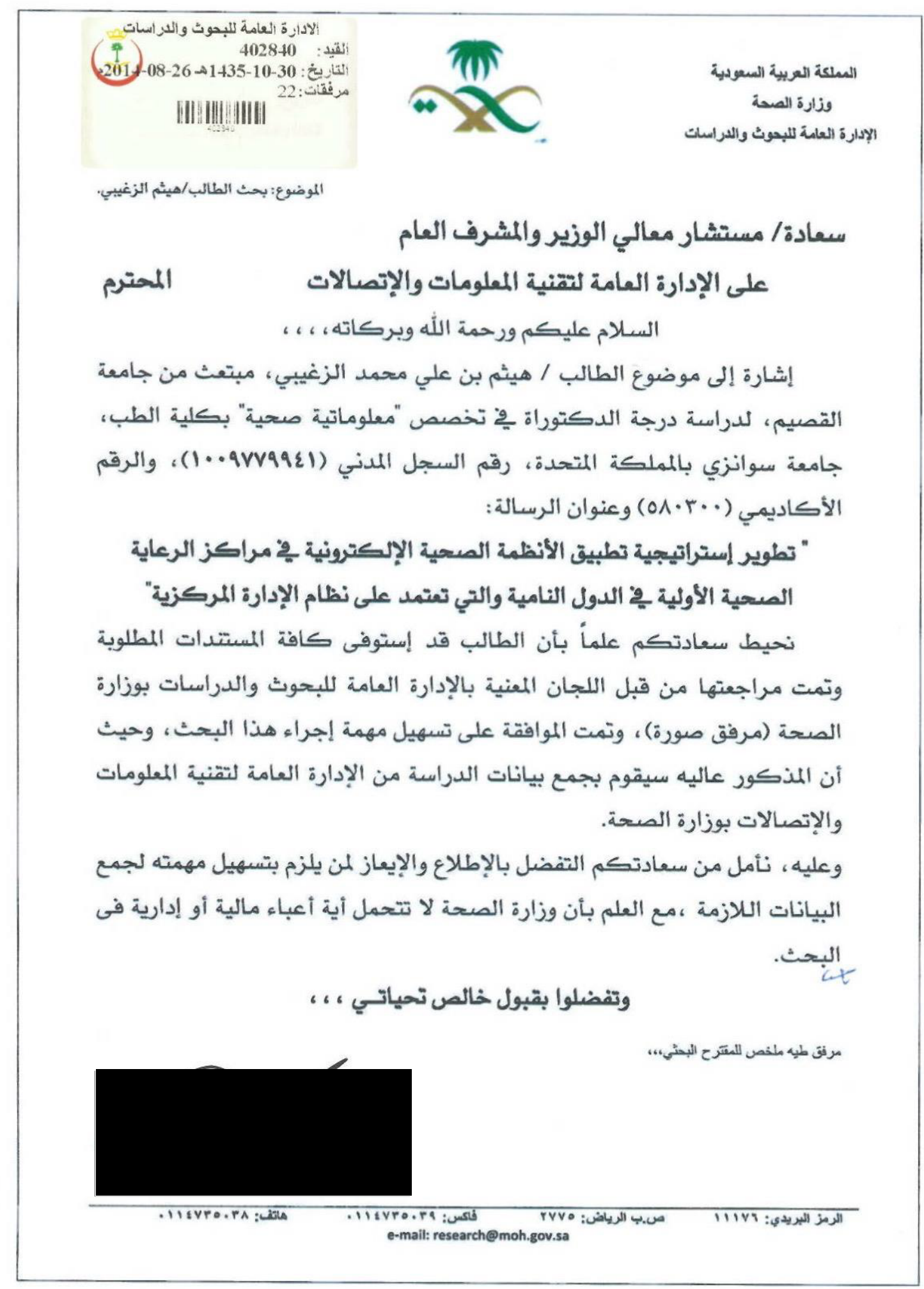




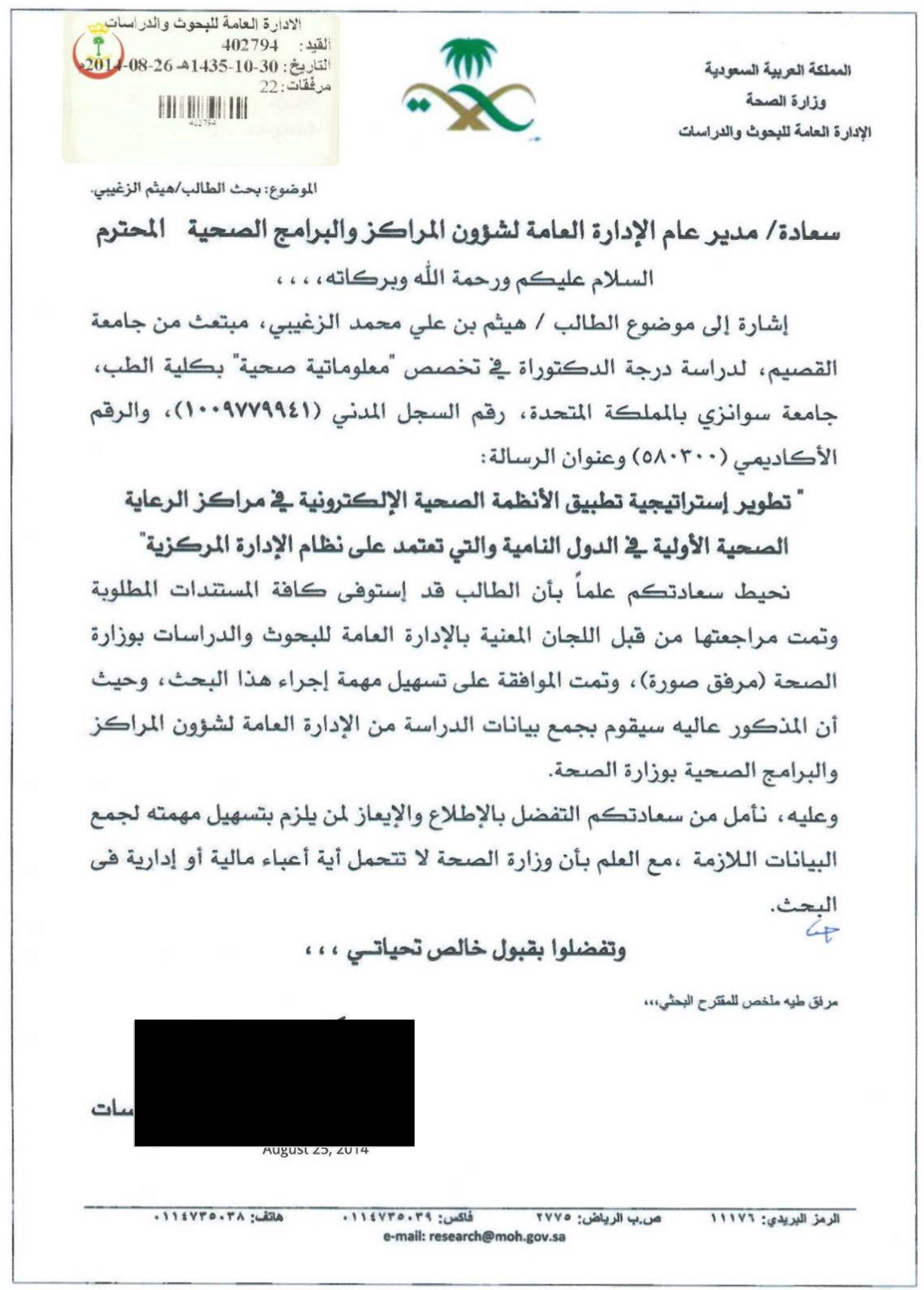




\subsection{Data collection instrument for Study One}

\section{$\underline{\text { Assurance letter }}$}

Research title: IMPLEMENTATION OF THE ELECTRONIC HEALTH RECORD SYSTEMS INPRIMARY HEALTHCARE CENTRES IN SAUDI ARABIA

You receive this questionnaire because you are currently involved in an Electronic Health Record system (EHRs) implementation. The questionnaire is part of a research study to assess Primary Healthcare Centres (PHCs) readiness to the implementation of the EHRS. This questionnaire includes question about your professional background, how much you come into contact with EHRs implementation, and how effective you perceive that it is. A high response rate increases the validity of the study. Answering the survey is estimated to take between 10 to 20 minutes.

Voluntary participation: It is voluntary to participate in the study and you can refrain from participation at any time without having to explain why. There are no known benefits or risks for you in this study.

Confidentiality: The researcher will handle the data anonymously and with confidentiality. Access to primary reported material is restricted to the researcher only (your employer will not have access to your answers). Readers of reports from the study will not be able to identify individual responses.

Data security: The researcher will do the best of effort to ensure the security of your data by extracting all the needed information form the paper questionnaire and fill the data into the appropriate software (e.g. SPSS). The researcher will lock the laptop with strong password and strong internet protection software will be installed.

Please do not hesitate to ask any questions.

Haitham Ali M. Alzghaibi

United Kingdom

Swansea University

$\mathrm{PhD}$ in Health informatics

Email:

Tel.

Organisational Information Technology/Systems Innovation Readiness Scale (OITIRS) 
Directions: For each statement, please circle the number of the one response that best reflects your personal opinion.

KEY: SD = Strongly Disagree, SA = Strongly Agree

Part One: PHCs readiness to the implementation of the EHRS:

\begin{tabular}{|c|c|c|c|c|c|c|c|c|}
\hline NO. & Statements & 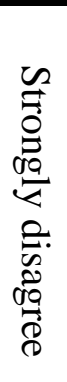 & 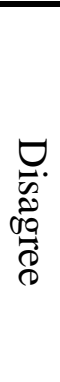 & 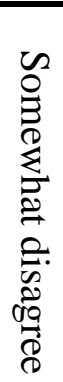 & $\begin{array}{l}z \\
\text { Z } \\
0 \\
0 \\
0 \\
\text { : }\end{array}$ & 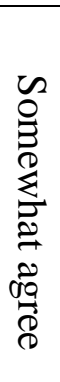 & $\underset{\substack{D \\
\mathbb{0}}}{\stackrel{D}{8}}$ & 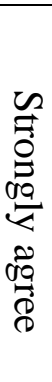 \\
\hline 1. & $\begin{array}{l}\text { Funding is adequate for completion of } \\
\text { EHRs implementation. }\end{array}$ & 1 & 2 & 3 & 4 & 5 & 6 & 7 \\
\hline 2. & $\begin{array}{l}\text { Project teams have included both } \\
\text { technical support staff and users. }\end{array}$ & 1 & 2 & 3 & 4 & 5 & 6 & 7 \\
\hline 3. & $\begin{array}{l}\text { The project budget includes } \\
\text { training/retraining costs. }\end{array}$ & 1 & 2 & 3 & 4 & 5 & 6 & 7 \\
\hline 4. & $\begin{array}{l}\text { The project budget is consistent with the } \\
\text { organisation's strategic plan. }\end{array}$ & 1 & 2 & 3 & 4 & 5 & 6 & 7 \\
\hline 5. & $\begin{array}{l}\text { There is a good ratio of full-time in-house } \\
\text { to contract IT staff to support the project. }\end{array}$ & 1 & 2 & 3 & 4 & 5 & 6 & 7 \\
\hline 6. & $\begin{array}{l}\text { Good quality vendor support for the EHRs } \\
\text { is typically available. }\end{array}$ & 1 & 2 & 3 & 4 & 5 & 6 & 7 \\
\hline 7. & $\begin{array}{l}\text { Most users have an adequate level of } \\
\text { computer literacy. }\end{array}$ & 1 & 2 & 3 & 4 & 5 & 6 & 7 \\
\hline 8. & Users are typically supportive of EHRs. & 1 & 2 & 3 & 4 & 5 & 6 & 7 \\
\hline 9. & $\begin{array}{l}\text { User competencies are appropriately } \\
\text { incorporated into job performance criteria. }\end{array}$ & 1 & 2 & 3 & 4 & 5 & 6 & 7 \\
\hline 10. & $\begin{array}{l}\text { Users are typically involved in EHRs } \\
\text { implementation. }\end{array}$ & 1 & 2 & 3 & 4 & 5 & 6 & 7 \\
\hline 11. & $\begin{array}{l}\text { Adequate training is available to support } \\
\text { users. }\end{array}$ & 1 & 2 & 3 & 4 & 5 & 6 & 7 \\
\hline 12. & $\begin{array}{l}\text { A core group of users (champions) is } \\
\text { available to support implementation. }\end{array}$ & 1 & 2 & 3 & 4 & 5 & 6 & 7 \\
\hline 13. & $\begin{array}{l}\text { Current work practices are adequately } \\
\text { supported by existing information } \\
\text { systems. }\end{array}$ & 1 & 2 & 3 & 4 & 5 & 6 & 7 \\
\hline 14. & $\begin{array}{l}\text { There is a good fit between organisational } \\
\text { and EHRs implementation strategic plans. }\end{array}$ & 1 & 2 & 3 & 4 & 5 & 6 & 7 \\
\hline 15. & $\begin{array}{l}\text { Research and development activities to } \\
\text { learn about new technology are supported. }\end{array}$ & 1 & 2 & 3 & 4 & 5 & 6 & 7 \\
\hline 16. & $\begin{array}{l}\text { EHRs project implementation time frames } \\
\text { are usually adequate. }\end{array}$ & 1 & 2 & 3 & 4 & 5 & 6 & 7 \\
\hline 17. & $\begin{array}{l}\text { Development of information systems is } \\
\text { based on current market trends. }\end{array}$ & 1 & 2 & 3 & 4 & 5 & 6 & 7 \\
\hline
\end{tabular}




\begin{tabular}{|c|c|c|c|c|c|c|c|c|}
\hline NO. & Statements & 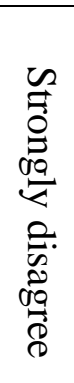 & 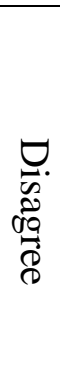 & 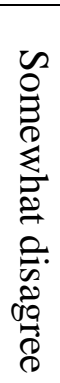 & $\begin{array}{l}z \\
\text { Z } \\
o \\
o \\
\text { o. } \\
\text { o. }\end{array}$ & 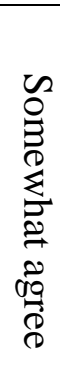 & $\underset{\substack{\infty \\
\overparen{D}}}{\stackrel{D}{0}}$ & 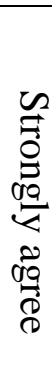 \\
\hline 18. & There are good quality vendor contracts. & 1 & 2 & 3 & 4 & 5 & 6 & 7 \\
\hline 19. & $\begin{array}{l}\text { There is a lot of knowledge about EHRs } \\
\text { operational and capital budget trends. }\end{array}$ & 1 & 2 & 3 & 4 & 5 & 6 & 7 \\
\hline 20. & $\begin{array}{l}\text { Historically, the strategic and EHRs } \\
\text { implementation goals have been } \\
\text { integrated. }\end{array}$ & 1 & 2 & 3 & 4 & 5 & 6 & 7 \\
\hline 21. & $\begin{array}{l}\text { In the past, EHRs users have been } \\
\text { included in decision-making processes. }\end{array}$ & 1 & 2 & 3 & 4 & 5 & 6 & 7 \\
\hline 22. & $\begin{array}{l}\text { Administrators are very knowledgeable } \\
\text { about EHRs based on their past } \\
\text { experience. }\end{array}$ & 1 & 2 & 3 & 4 & 5 & 6 & 7 \\
\hline 23. & $\begin{array}{l}\text { There is a lot of knowledge about the on- } \\
\text { going development needs of EHRs } \\
\text { support staff. }\end{array}$ & 1 & 2 & 3 & 4 & 5 & 6 & 7 \\
\hline 24. & $\begin{array}{l}\text { Knowledge is available about how EHRs } \\
\text { implementation is being used by other } \\
\text { organisations. }\end{array}$ & 1 & 2 & 3 & 4 & 5 & 6 & 7 \\
\hline 25. & $\begin{array}{l}\text { Adequate communication mechanisms } \\
\text { exist to support shared communication } \\
\text { across all organisational levels. }\end{array}$ & 1 & 2 & 3 & 4 & 5 & 6 & 7 \\
\hline 26. & $\begin{array}{l}\text { Effective mechanisms are in place to } \\
\text { evaluate EHRs implementation. }\end{array}$ & 1 & 2 & 3 & 4 & 5 & 6 & 7 \\
\hline 27. & $\begin{array}{l}\text { The most appropriate individuals are } \\
\text { involved in the development of the EHRs } \\
\text { implementation strategic plan. }\end{array}$ & 1 & 2 & 3 & 4 & 5 & 6 & 7 \\
\hline 28. & $\begin{array}{l}\text { EHRs implementation needs are routinely } \\
\text { incorporated into the organisation's } \\
\text { business processes. }\end{array}$ & 1 & 2 & 3 & 4 & 5 & 6 & 7 \\
\hline 29. & $\begin{array}{l}\text { Process improvement mechanisms are } \\
\text { used effectively to identify work process } \\
\text { redesign needs. }\end{array}$ & 1 & 2 & 3 & 4 & 5 & 6 & 7 \\
\hline 30. & $\begin{array}{l}\text { EHRs implementation decision makers } \\
\text { are adequately represented on key } \\
\text { organisational committees. }\end{array}$ & 1 & 2 & 3 & 4 & 5 & 6 & 7 \\
\hline 31. & $\begin{array}{l}\text { There is a willingness to act on work } \\
\text { process improvement recommendations. }\end{array}$ & 1 & 2 & 3 & 4 & 5 & 6 & 7 \\
\hline 32. & $\begin{array}{l}\text { There is satisfaction with the contribution } \\
\text { that EHRs has made to the organisation. }\end{array}$ & 1 & 2 & 3 & 4 & 5 & 6 & 7 \\
\hline 33. & $\begin{array}{l}\text { There is an openness to different } \\
\text { perspectives about EHRs implementation. }\end{array}$ & 1 & 2 & 3 & 4 & 5 & 6 & 7 \\
\hline
\end{tabular}




\begin{tabular}{|c|c|c|c|c|c|c|c|c|}
\hline NO. & Statements & 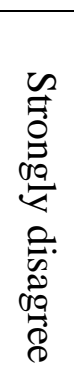 & 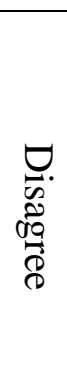 & $\begin{array}{l}2 \\
0 \\
0 \\
0 \\
0 \\
0 \\
0 \\
0 \\
0 \\
0 \\
0 \\
0 \\
0 \\
0\end{array}$ & $\begin{array}{l}z \\
\text { Z } \\
0 \\
0 \\
0 \\
0 \\
0\end{array}$ & 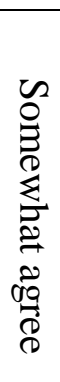 & 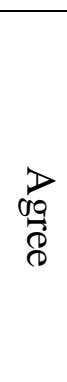 & 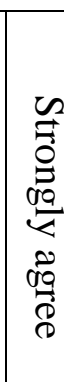 \\
\hline 34. & $\begin{array}{l}\text { There is an emphasis on the importance of } \\
\text { collaborative interdisciplinary teams to } \\
\text { support EHR implementation. }\end{array}$ & 1 & 2 & 3 & 4 & 5 & 6 & 7 \\
\hline 35. & $\begin{array}{l}\text { There is a willingness to engage in the } \\
\text { EHRs implementation process. }\end{array}$ & 1 & 2 & 3 & 4 & 5 & 6 & 7 \\
\hline 36. & $\begin{array}{l}\text { Individuals have a positive attitude toward } \\
\text { EHRs implementation. }\end{array}$ & 1 & 2 & 3 & 4 & 5 & 6 & 7 \\
\hline 37. & $\begin{array}{l}\text { The business structure supports } \\
\text { involvement of IS in strategic planning. }\end{array}$ & 1 & 2 & 3 & 4 & 5 & 6 & 7 \\
\hline 38. & $\begin{array}{l}\text { Formal communication mechanisms exist } \\
\text { to support user and IT support staff } \\
\text { communication. }\end{array}$ & 1 & 2 & 3 & 4 & 5 & 6 & 7 \\
\hline 39. & $\begin{array}{l}\text { The IT department reporting structure } \\
\text { adequately supports IT staff. }\end{array}$ & 1 & 2 & 3 & 4 & 5 & 6 & 7 \\
\hline 40. & $\begin{array}{l}\text { The IT strategic plan is an effective guide } \\
\text { for the organisation's EHRs } \\
\text { implementation processes. }\end{array}$ & 1 & 2 & 3 & 4 & 5 & 6 & 7 \\
\hline 41. & $\begin{array}{l}\text { The IT department effectively manages } \\
\text { the organisation's shared databases. }\end{array}$ & 1 & 2 & 3 & 4 & 5 & 6 & 7 \\
\hline 42. & $\begin{array}{l}\text { Formal policies and procedures are } \\
\text { available to guide EHRs implementation } \\
\text { processes. }\end{array}$ & 1 & 2 & 3 & 4 & 5 & 6 & 7 \\
\hline 43. & $\begin{array}{l}\text { EHRs implementation initiatives are } \\
\text { usually addressed as part of the } \\
\text { organisation's overall strategic planning. }\end{array}$ & 1 & 2 & 3 & 4 & 5 & 6 & 7 \\
\hline 44. & $\begin{array}{l}\text { Board members are actively engaged in } \\
\text { key EHRs implementation strategic plan } \\
\text { committees. }\end{array}$ & 1 & 2 & 3 & 4 & 5 & 6 & 7 \\
\hline 45. & $\begin{array}{l}\text { Sufficient funds are available to support } \\
\text { EHRs implementation planning activities. }\end{array}$ & 1 & 2 & 3 & 4 & 5 & 6 & 7 \\
\hline 46. & $\begin{array}{l}\text { The top-ranking IT executive is regularly } \\
\text { included in senior executive meetings. }\end{array}$ & 1 & 2 & 3 & 4 & 5 & 6 & 7 \\
\hline 47. & $\begin{array}{l}\text { Non-IT executives are routinely named as } \\
\text { co-sponsors for EHRs implementation } \\
\text { projects. }\end{array}$ & 1 & 2 & 3 & 4 & 5 & 6 & 7 \\
\hline 48. & $\begin{array}{l}\text { Executives engage in mutual decision- } \\
\text { making with-IT leaders regarding } \\
\text { proposals and ideas. }\end{array}$ & 1 & 2 & 3 & 4 & 5 & 6 & 7 \\
\hline
\end{tabular}

Part Two: Description of other factors influencing the implementation: 
- Centralisation Management, does this system influence the EHRs implementation in the PHCS with respect to the below aspects:

\begin{tabular}{|c|c|c|c|c|c|c|c|c|}
\hline NO. & Aspects & 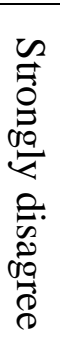 & 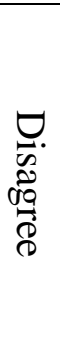 & 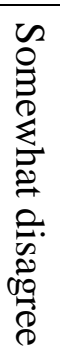 & 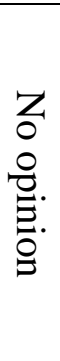 & 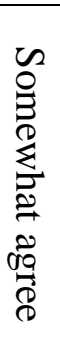 & 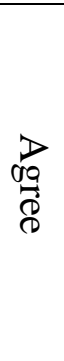 & 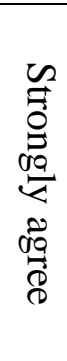 \\
\hline 49. & Improve implementation strategic plan. & 1 & 2 & 3 & 4 & 5 & 6 & 7 \\
\hline 50. & $\begin{array}{l}\text { Easier to manage the EHRs } \\
\text { implementation in large PHCS number, } \\
\text { and the long distance between them. }\end{array}$ & 1 & 2 & 3 & 4 & 5 & 6 & 7 \\
\hline 51. & Better decision making. & 1 & 2 & 3 & 4 & 5 & 6 & 7 \\
\hline 52. & Better software selection. & 1 & 2 & 3 & 4 & 5 & 6 & 7 \\
\hline 53. & Better team selection. & 1 & 2 & 3 & 4 & 5 & 6 & 7 \\
\hline 54. & Improve team communication. & 1 & 2 & 3 & 4 & 5 & 6 & 7 \\
\hline 55. & Help leading and managing the project. & 1 & 2 & 3 & 4 & 5 & 6 & 7 \\
\hline 56. & $\begin{array}{l}\text { Improve systems integration and } \\
\text { interoperability. }\end{array}$ & 1 & 2 & 3 & 4 & 5 & 6 & 7 \\
\hline 57. & Overall impact is positive. & 1 & 2 & 3 & 4 & 5 & 6 & 7 \\
\hline
\end{tabular}

- Financial Resources; does this factor influences the EHRs implementation in the PHCS with respect to the below aspects:

\begin{tabular}{|c|c|c|c|c|c|c|c|c|}
\hline NO. & Aspects & 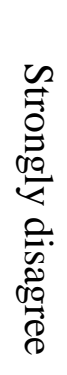 & 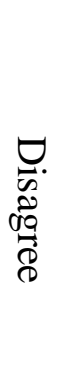 & 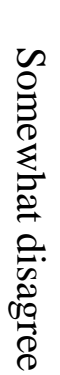 & $\begin{array}{l}z \\
\text { o } \\
o \\
\text { o } \\
\text { E. } \\
\text { o. }\end{array}$ & 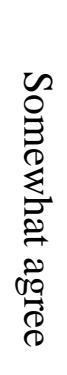 & 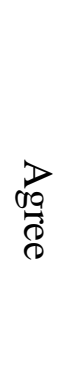 & 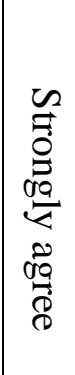 \\
\hline 58. & Better software selection. & 1 & 2 & 3 & 4 & 5 & 6 & 7 \\
\hline 59. & Better team selection. & 1 & 2 & 3 & 4 & 5 & 6 & 7 \\
\hline 60. & Improve the team communication. & 1 & 2 & 3 & 4 & 5 & 6 & 7 \\
\hline 61. & $\begin{array}{l}\text { Improve organisation's workflow and } \\
\text { structure redesign }\end{array}$ & 1 & 2 & 3 & 4 & 5 & 6 & 7 \\
\hline
\end{tabular}




\begin{tabular}{|c|c|c|c|c|c|c|c|c|}
\hline NO. & Aspects & 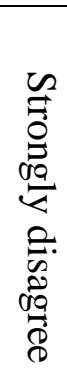 & 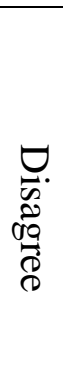 & $\begin{array}{c}\tilde{2} \\
0 \\
0 \\
0 \\
0 \\
0 \\
0 \\
0 \\
0 \\
0 \\
0 \\
0 \\
0 \\
0\end{array}$ & 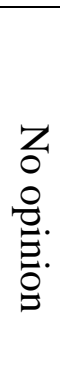 & 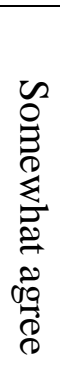 & 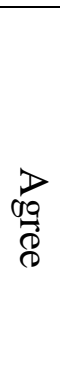 & 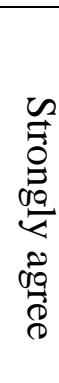 \\
\hline 62. & Appropriate infrastructure. & 1 & 2 & 3 & 4 & 5 & 6 & 7 \\
\hline 63. & $\begin{array}{l}\text { Improve the on-going support and } \\
\text { maintenance. }\end{array}$ & 1 & 2 & 3 & 4 & 5 & 6 & 7 \\
\hline 64. & $\begin{array}{l}\text { Improve the provision of appropriate } \\
\text { hardware. }\end{array}$ & 1 & 2 & 3 & 4 & 5 & 6 & 7 \\
\hline 65. & Improve users training and motivation. & 1 & 2 & 3 & 4 & 5 & 6 & 7 \\
\hline 66. & Abundance in staff and professionals. & 1 & 2 & 3 & 4 & 5 & 6 & 7 \\
\hline 67. & $\begin{array}{l}\text { Improve systems integration and } \\
\text { interoperability. }\end{array}$ & 1 & 2 & 3 & 4 & 5 & 6 & 7 \\
\hline 68. & Overall impact is positive. & 1 & 2 & 3 & 4 & 5 & 6 & 7 \\
\hline
\end{tabular}

Part Three: Your Responses are completely anonymous, but I would like to ask you a few questions about who you are:

69. Gender: $\square$ Female $\square$ Male

70. Your position (as of $19 / 10 / 2014$ ):

71. Your role in the EHRs implementation:

72. Have you involved in similar EHRs implementation projects before?

Yes $\square$ NO 
Welcome to My Survey

تعهدات الباحث

عنوان البحث: تطوير استر اتيجية تطبيق الإظمة الصحية الاكترونية في مراكز الرعاية الصحية المنتشره في الدول النامية والتي تطبق نظام الإدارة

تم ارسال هذه الاستبانة لاكك حالياً تعمل في مر اكز الرعاية الصحية الاولية و التي سوف يتم تطبيق الانظمة الاكترونية فيها. الاستبيان جزء من

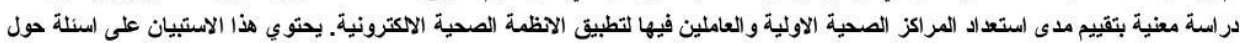

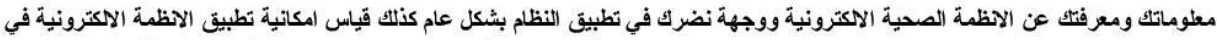

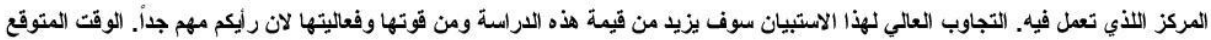
للاجابة على هذا الاستيبان هو مابين 10 إلى 20 دقيقه فقط. لهُب.

تتعبر المشاركة في هذا الاستبيان اختيارية وليست اجبارية ولك الحقى في رفض المشاركة في اي وقت تثاء ولك الحقى في عدم ذكر المبررات. لا توجل مميز ات مادية للمشاركة في هذا الاستبيان

الباحث سوف يتعامل مع هذه البيانات بلدن اسماء وبسرية تامه. وسوف يكون الاطلاع على الاستبيان والبيانات الاولية محصورأ على الباحث نفسة

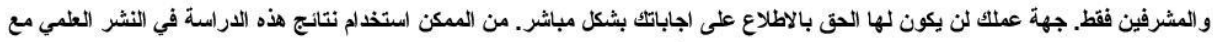

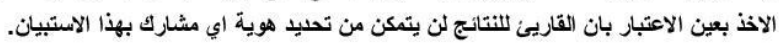

الباحث سوف يتخذ جميع الاجر اعات المناسبة لحماية البيانات عن طريق استخر اج البيانات المطلوبه من الاستبيان وتفريغها في برنامج الحزم

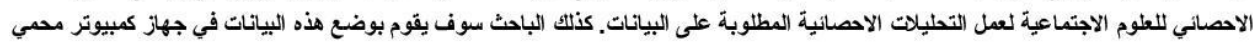
ببرنامج حماية ضد الاختر اق.

لا تتردد بالتواصل مع الباحث او احد المشرفين لطرح اي سؤال او استفسار

هيثم علي محمد الززيبي

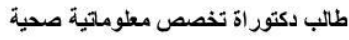
جامعة سو انزي

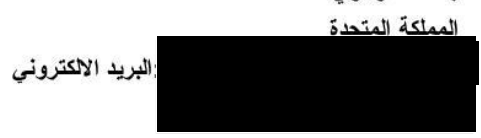

المشرف الرئيسي د.قارث نويل

البريد الأكتروني

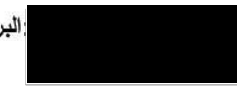

المشرف الثاني

دـ. ميلني هيلي

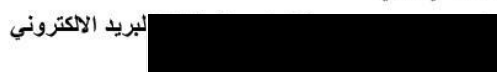


Reserach title: Improve strategy to implement the Electronic Health Records Systems on Widespread Primary Healthcare Centres in developing countries with centralised management systems organisation.

You receive this questionnaire because you are currently working in Primary Healthcare Centres (PHC). The questionnaire is part of a research study to evaluate the readiness of the PHC to the EHRs implementation.

This questionnaire includes question about your professional background and your perception on the introduction of the EHRs. A high response rate increases the validity of the study. Answering the survey is estimated to take between 10 to 20 minutes.

Voluntary participation: It is voluntary to participate in the study and you can refrain from participation at any time without having to explain why. There are no known benefits or risks for you in this study.

Confidentiality: The researcher will handle the data anonymously and with confidentiality. Access to primary reported material is restricted to the researcher only (your employer will not have access to your answers). Readers of reports from the study will not be able to identify individual responses.

Data security: The researcher will do the best of effort to insure the security of your data by extracting all the needed information form the paper questionnaire and fill the data into the appropriate software (eg. SPSS). The researcher will locked the laptop with strong password and strong internet protection software will be installed.

Please do not hesitate to ask any questions.

Haitham Ali M. Alzghaibi

Main Supervisor

Dr Gareth Noble

inited Kingdom

Swansea University

PhD Candidate

Email:

Mobile No.
Email:

Tel:
Second Supervisor

Dr Melanie A. Healy

Email:

Tel: 
1. What is the name of the region

ماهي اسم المنطقة الإدارية التابع لها؟

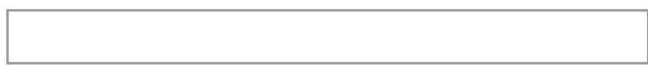

2. What is your role?

طبيب Physician

مدير المركز Manager

ممرض Nurse

فني مختبر Lap technician

صنيلي Pharmacist

فني الشعة Radiologist

طبيب اسنان Dentist

موظف اسنقبال Receptionist

Other (please specify) إخرى أمل نكرها

3. What is your age?

العمر؟

18 to 24

25 to 34

35 to 44

45 to 54

55 to 64

( 65 to 74

75 or older

4. What is your gender?

الجنس

أنثى Female

ن Male 


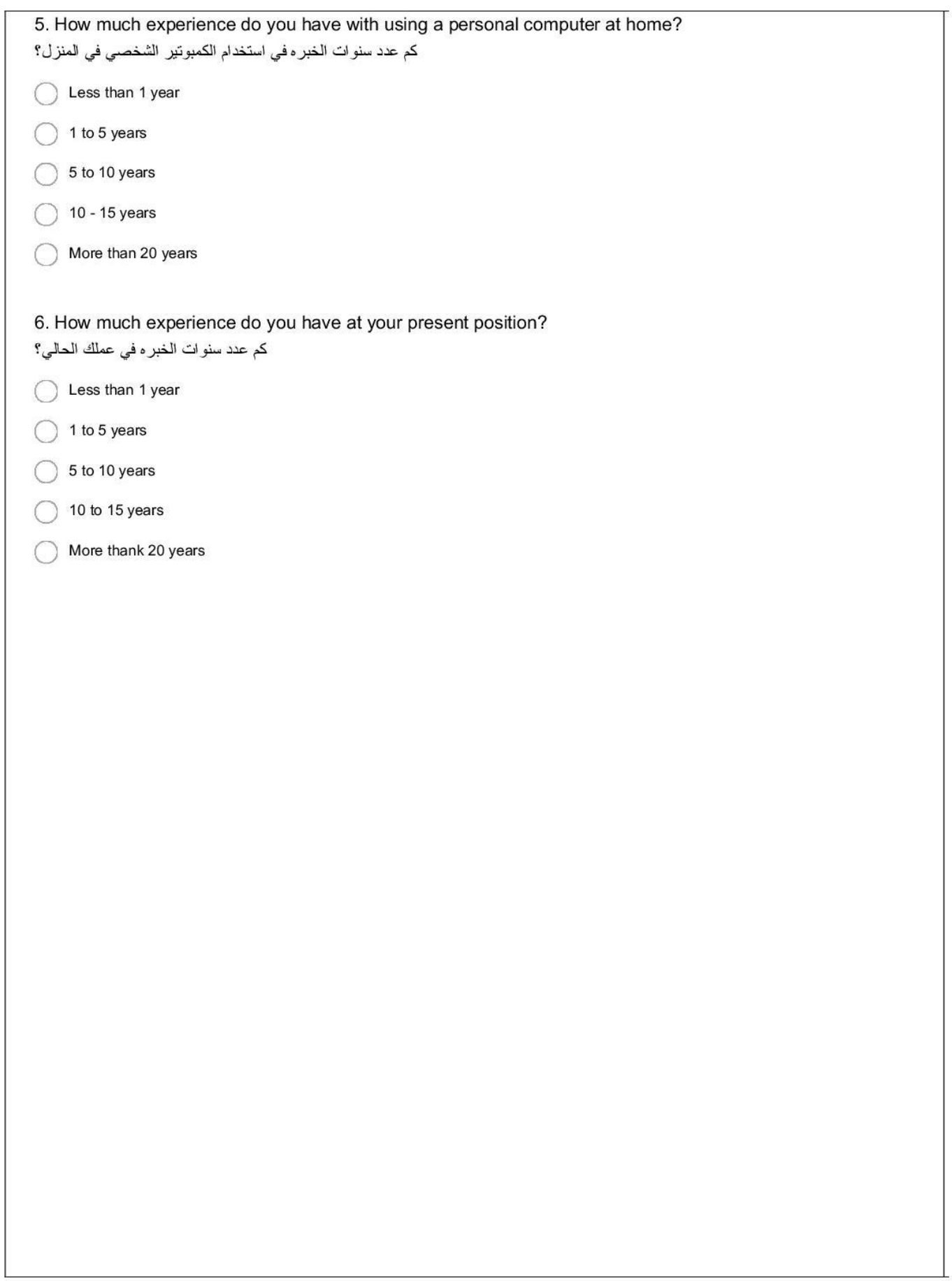


7. The Benefits of Electronic Health Records فو اند استخدام النظام الصحي الالكتروني

$\begin{array}{llll}\text { Strongly Disagree Disagree } & \text { Agree } & \text { Strongly Agree opinion }\end{array}$

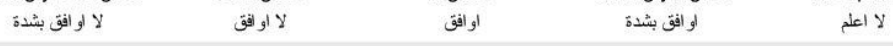

\section{The EHRs provide}

accurate, up-to-date,

and complete

information about

patients at the point of

care

النظام الصحي الالكثروني يعطي

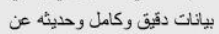

لمريض اثناء العلاج و التئنيص

Information from the

EHRs enables me to

make better decisions

about patient care.

المعلومات اللتي احصل عليها من

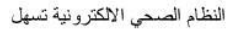

تخاذ القرار المناسب لخدمة وعلاج

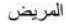

The EHRs enable quick

access to patient

records for more

coordinated, efficient

care

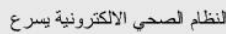

علية الحصول على ملف المريض المريض

لتو فير خدمة اكفاء و اسر ع للمريض المريض

The EHRs enable quick

access to patient

records for more

coordinated, efficient

care

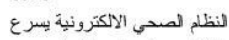

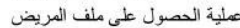

لتوفير خذمة اكفاء و اسر ع للمريض لطريض

Sharing electronic

information with patients

and other clinicians is

more secure when using

the EHRs

مشاركة بيانات المرضى من خلال

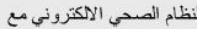

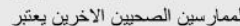

كثر امناً 


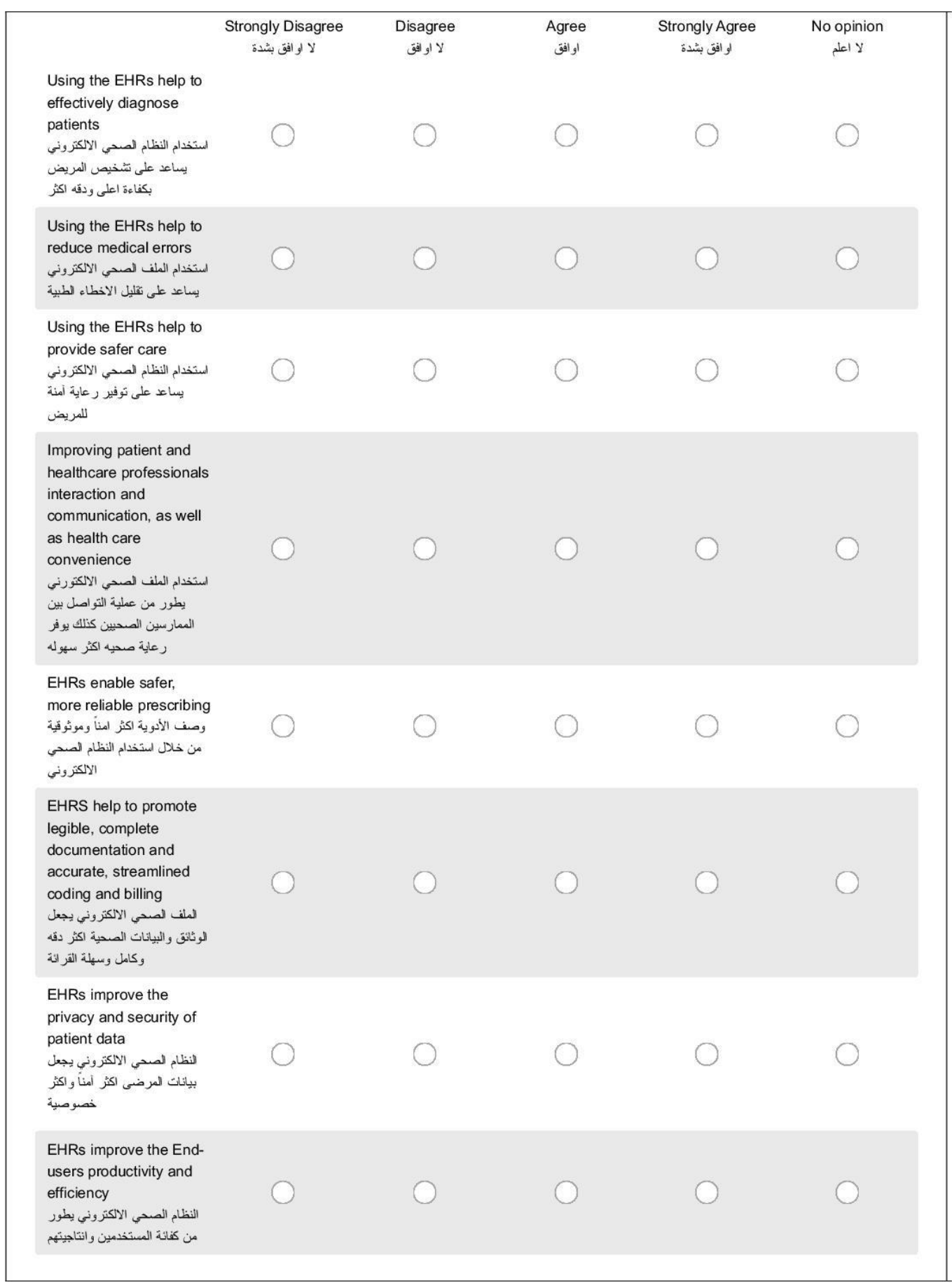




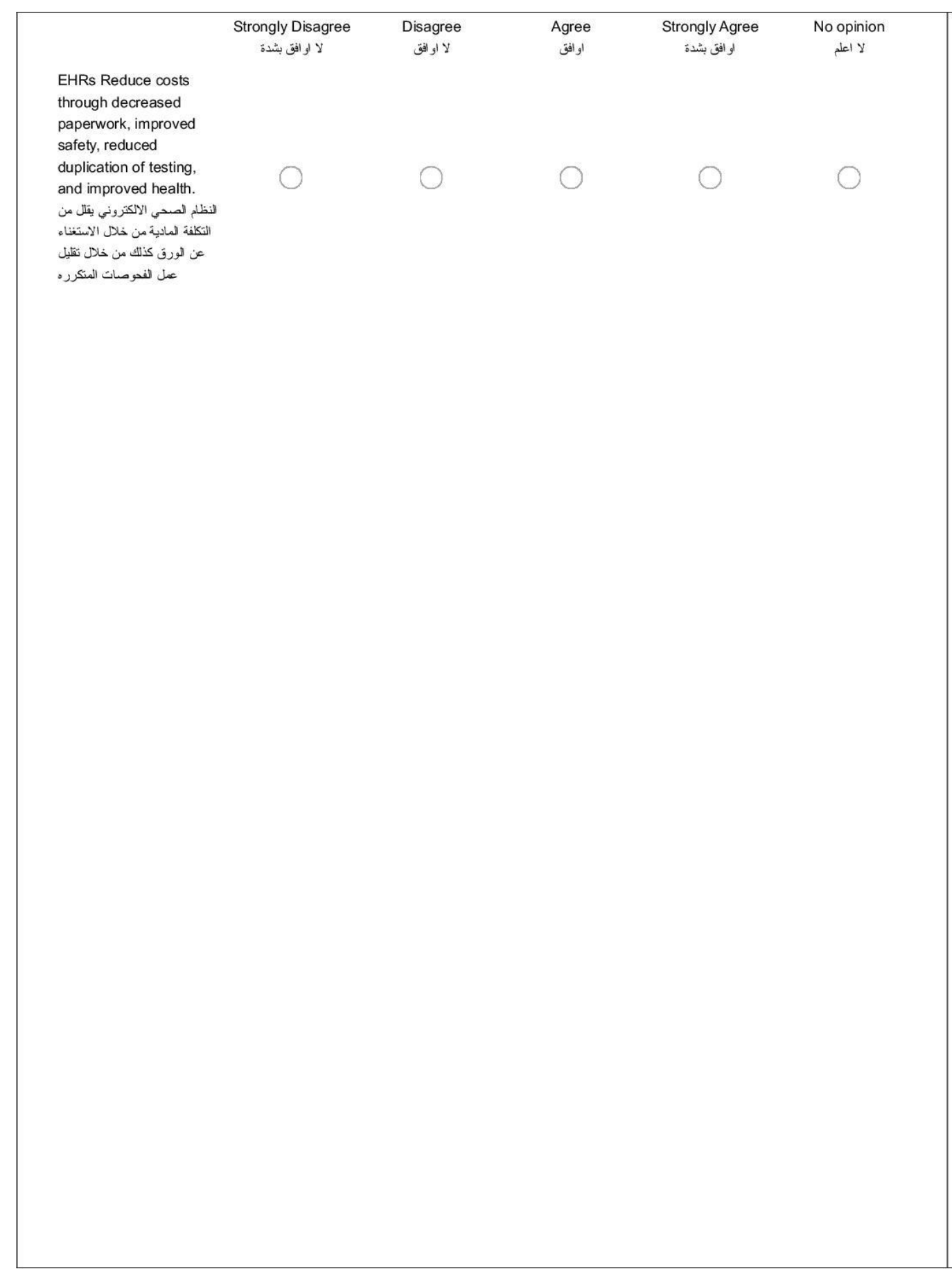


8. Measure your organisation readiness to implement the EHRs

قياس مد ى استعداد مر اكز الرعاية الصحية لنطبيق الانظمة الصحية الاكترونية

\begin{tabular}{|c|c|c|c|c|}
\hline $\begin{array}{c}\text { Strongly Disagree } \\
\text { لا او افقت بشدة: }\end{array}$ & $\begin{array}{c}\text { Disagree } \\
\text { لاواوفق }\end{array}$ & 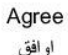 & $\begin{array}{c}\text { Strongly Agree } \\
\text { او افق بشدة }\end{array}$ & No Opinion \\
\hline
\end{tabular}

Most users have an

adequate level of

computer literacy.

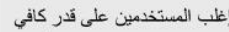

من المعمرفة في استخدام أجزة:

الكبيوتز

Users are typically

supportive of EHRs.

العاملين داعمين ولديهي رغبه في

تطبيق الانظمة الالكترونية

Users are typically

involved in EHRs

implementation.

المستخدمين و العاملين في المركز

علاة بتم الثر اكهم في عملية تطبيق الكريق

الانظمة الاككرونية

Adequate training is

available to support

users.

توفر التكريب المناسب لدعم

المستخدمين لاستخدام الانظمة لإنة

الالكترونية الانية

A core group of users

(champions) is available

to support

implementation

تم تعيين مجمو عة من الموظفين

العاملين في لمركز نفسه للاشر الف الفرن

ودعم تطبيق الانظمة الاككترونية

Current work practices

are adequately

supported by existing

information systems.

الية العمل الحالية بالمركز مدعومة

باستخذام نظام صدي الكتروني بالئي

همتوفر حاليًا

Staff have been included

in decision-making

processes.

اعضاء المركز تم الشر اكهم في

عملية اتخلا القرار تات في عملية

تطبيق الانظمة الصحية الالكتورنية 


\begin{tabular}{|c|c|c|c|c|c|}
\hline & $\begin{array}{l}\text { Strongly Disagree } \\
\text { لا وا افق بشدة: }\end{array}$ & $\begin{array}{l}\text { Disagree } \\
\text { لا او الفق }\end{array}$ & $\begin{array}{l}\text { Agree } \\
\text { اوافق }\end{array}$ & $\begin{array}{c}\text { Strongly Agree } \\
\text { لوا وافق بشدة }\end{array}$ & $\begin{array}{l}\text { No Opinion } \\
\text { ע l|ll }\end{array}$ \\
\hline 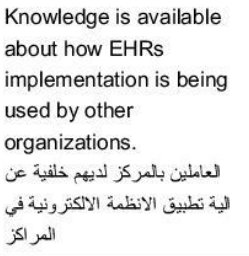 & 0 & 0 & 0 & ) & ) \\
\hline 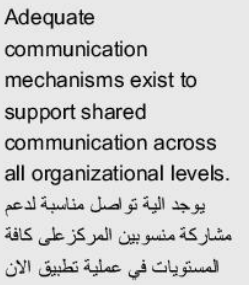 & & & & & \\
\hline $\begin{array}{l}\text { There is an emphasis } \\
\text { on the importance of } \\
\text { collaborative } \\
\text { interdisciplinary teams } \\
\text { to support EHR } \\
\text { implementation. } \\
\text { im التعاون مع العلين مخلف التركخ }\end{array}$ & & & & & \\
\hline 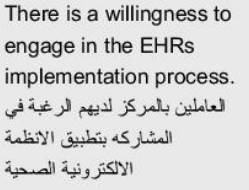 & & & & & \\
\hline 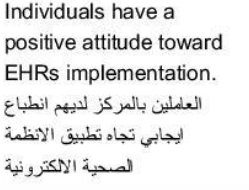 & & & & & \\
\hline 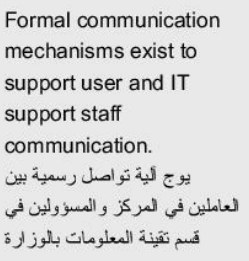 & & 0 & 0 & 0 & 0 \\
\hline
\end{tabular}


9. In general, do you endorses the implementation the EHRs in the PHC?

بشكل عام هل تؤيد تطبيق الانظمة الصحية الاكترونية في مر اكز الرعاية الصحية؟

Yes rev

Noy

Why?

أرجو ذكر الاسباب؟

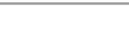

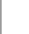

10. Would you be resistance to have EHRs?

هل سوف ترفض فكرة تطبيق الانظمة الصحية الالكترونية؟

Yes نer

Noy

11. Do you have any recommendations to the decision makers to improve the implementation of the EHRs?

هل لديك اية نصاتح أو مقترحات لمتخذين القرار بالوزارة لنظوير تطبيق و استخدام النظام الصحي الالكتروني؟ 
1. Welcome to My Survey

تعهدات الباحث

عنوان البحث: تطوير استر اتيجية تطبيق الانظمة الصحية الاكترونية في مر اكز الرعاية الصحية المنتشره في الدول النامية و التي تطبق نظام الإدارة

المركزية

تم ارسل هذه الاستبانة لاكك حالياً تستخدم النظام الصحي الاكتروني في مكان عملك بالمركز الصحي. الاستبيان جزء من دراسة معنية بتقيّييم الاظظمة

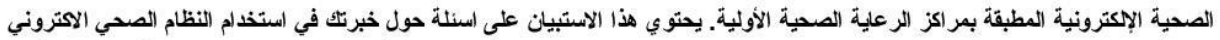

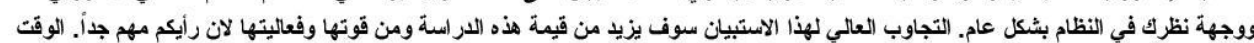
المتوقع للاجابة على هذا الاستيبان هو مابين 5 إلى 20 دقيقه فقط لأنطان.

تتعبر المشاركة في هذا الاستبيان اختيارية وليست اجبارية ولك الحق في رفض المشاركة في اي وقت تشاءو ولك الحق في عدم ذكر المبررات. لا

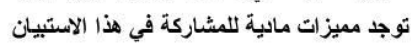

الباحث سوف يتعامل مع هذه البيانات بدون اسماء وبسرية تامه. وسوف يكون الاطلاع على الاستبيان و البيانات الاولية محصوراً على الباحث نفسة

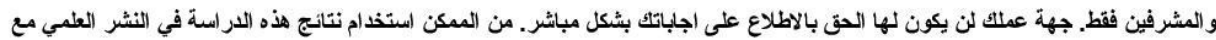

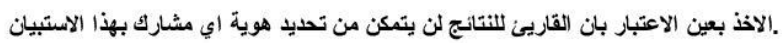

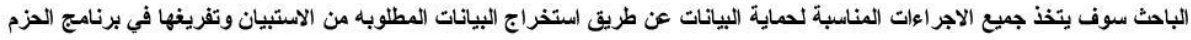

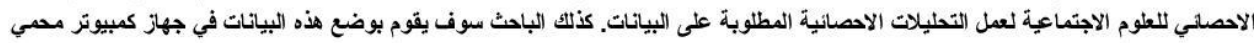
يبرنامج حماية ضد الاختراق

لا تتردد بالتواصل مع الباحث او احد المشرفين لطرح اي سؤل او استفسار هيثم علي محمد الز غيبي طالب دكتوراة تخصص معلوماتية صحية جامعة سوانزي الملكة المتحدة
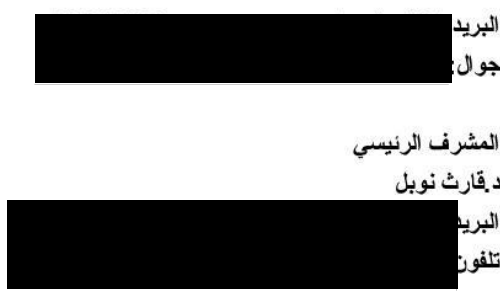

المشرف الثاني

د. ميلني هيلي

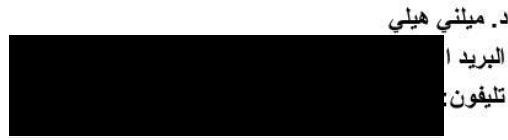


2. Welcome

\section{Assurance letter}

Research title: Improve strategy to implement the Electronic Health Records Systems in Widespread Primary Healthcare Centers in developing countries with centralised management systems organisation.

You have received the questionnaire because you are currently using the Electronic Health Record system (EHRs) in your workplace. The questionnaire is part of a research study to evaluate the EHRs implementation in Primary Healthcare Centres (PHC).

This questionnaire includes questions about your professional background and your experience with the EHRs implementation. However, a high response rate increases the validity of the study. Answering the survey is estimated to take between 5 to 20 minutes.

It is voluntary to participate in the study and you can refrain from participation at any time without having to explain why.

The researcher will handle the data anonymously and with confidentiality. Access to primary reported material is restricted to the researcher, the supervisory team and for publications (your employer will not have access to your answers). Readers of reports from the study will not be able to identify individual responses.

The researcher will ensure the security of your data by extracting all the needed information from the paper questionnaire and fill the data into the appropriate software. No data held on university server.

Please do not hesitate to ask any questions.

Haitham Ali M. Alzghaibi Main Superviso

United Kingdom

Swansea University

Email:

Ph.D. Candidate

Dr Gareth Noble

Email:

Email:

Mobile No
Tel:
Second Supervisor

Dr Melanie A. Healy

Tel: 
3. Demographics الاسئلة الديمو غر افية

The purpose of this section is to determine the participants characteristics and the differents between groups

الغرض من هذا الجزء هو لتحديد صفات المشاركين كنلك لمعرفة الاختلافات بين المشاركين

1. What is the name of the region in which you work?

ماهي اسم المنطقة الإدارية التابع لها؟

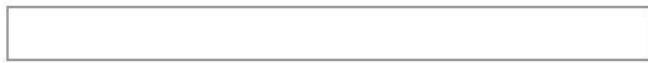

2. What is your job title?

ماهو مسمى وظيفتك؟

Physician

Manager مدير مركز

Nurse مaرض

فني مختبر Lap technician

Pharmacist صنيلي

Radiologist فن الشعة

Dentist طبيب اسنان

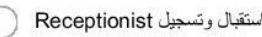

Other (please specify) أخرى (رجو ذكرها)

3. How much experience do you have with your present position? كم عدد سنوات الخبره في عملك الحالي؟

Less than 1 year

1 to 5 years

5 to 10 years

$10-15$ years

More than 20 years 


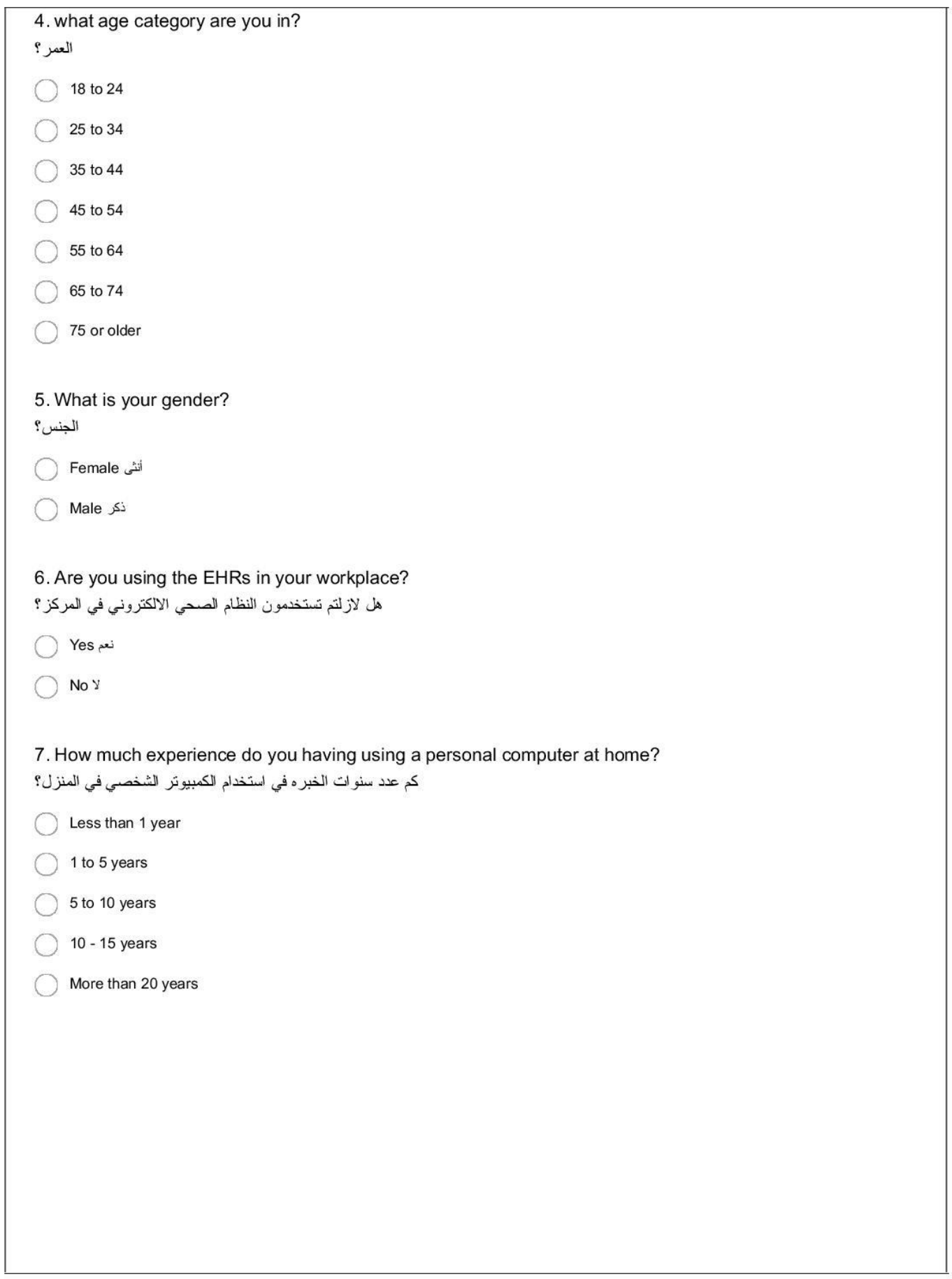




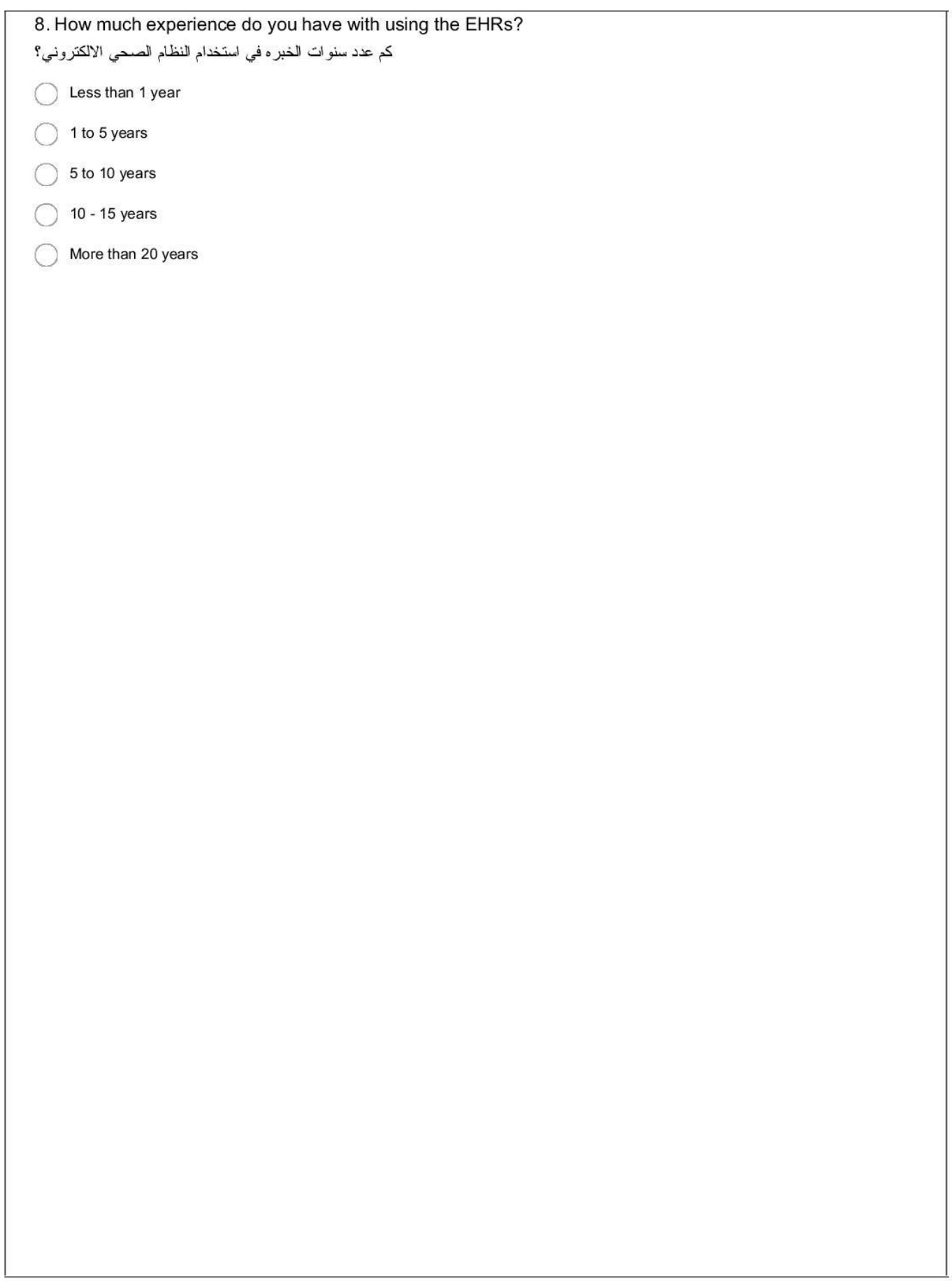


4. Evaluation of the benifites of the EHRs تقيييم مز ايا النظام الصحي الالكتروني

The purpose of this section is to identify the advantages of using the EHRs and its impact to the healthcare provided

الغرض من هذا القسم هو لمعرفة ايجابات استخدام النظام الصحي الاكتروني وتاثيره على الرعاية الصحية المقدمه

9. The Benefits of Electronic Health Records

\begin{tabular}{|c|c|c|c|c|}
\hline $\begin{array}{c}\text { Strongly Disagree } \\
\text { أوافق بشدة: }\end{array}$ & $\begin{array}{c}\text { Disagree } \\
\text { لا اوافق }\end{array}$ & $\begin{array}{c}\text { Neutral } \\
\text { محايذ }\end{array}$ & $\begin{array}{c}\text { Agree } \\
\text { أوافق }\end{array}$ & Strongly Agree \\
\hline
\end{tabular}

EHRs provide accurate,

up-to-date, and

complete information

about patients at the

point of care

النظام الصحي الاكتروني بعطي

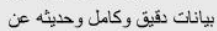

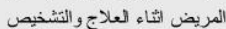

Information from the

EHRs enables me to

make better decisions

about patient care.

النظام الصدي الاكتروني يعطي

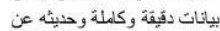

المريض ائثاء العلاج والتشخيص وطيثل

EHRs enable quick

access to patient

records for more

coordinated and efficient

care

لنظام الصدية الاكتروني يسرع عليق

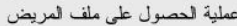

لتوفير خدمة اكفاء واسرع للمريض لمريض

EHRs allow me to spend

more time on other

aspects of patient care

النظام الصحي الالكثروني يوفر

وقت كبير وذلك يساعد باجر اءوناء

امور اخرى تخنص برعاية

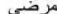

Sharing electronic

information with patients

and other clinicians is

more secure when using

the EHRs

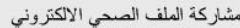

يطور من عملية التو اصل بين الاكترني

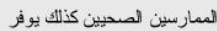

رعاية صحية اكثر سهولة لكئة 


\begin{tabular}{|c|c|c|c|c|c|}
\hline & $\begin{array}{c}\text { Strongly Disagree } \\
\text { لا أوافق بشدة: }\end{array}$ & $\begin{array}{c}\text { Disagree } \\
\text { لا اوافق }\end{array}$ & $\begin{array}{c}\text { Neutral } \\
\text { محايذ }\end{array}$ & $\begin{array}{c}\text { Agree } \\
\text { أوافق }\end{array}$ & $\begin{array}{c}\text { Strongly Agree } \\
\text { أو افق بشدة: }\end{array}$ \\
\hline 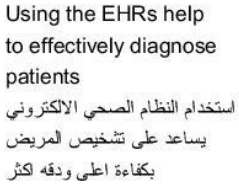 & & & & & \\
\hline 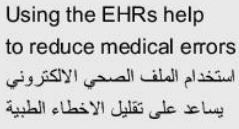 & & & & & \\
\hline 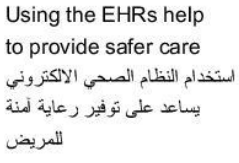 & & & & & \\
\hline 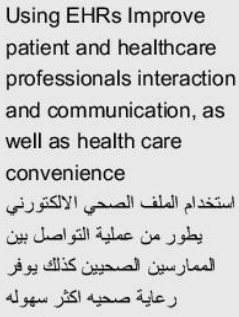 & & & & & \\
\hline 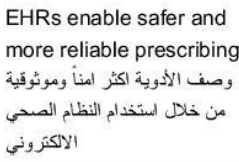 & & & & & \\
\hline 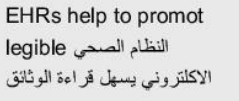 & & & & & \\
\hline 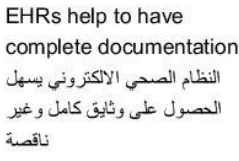 & & & & & \\
\hline 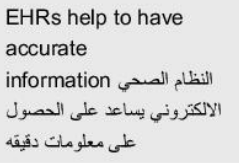 & & & & & \\
\hline 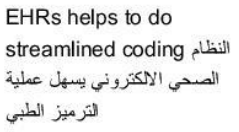 & & & & & \\
\hline
\end{tabular}




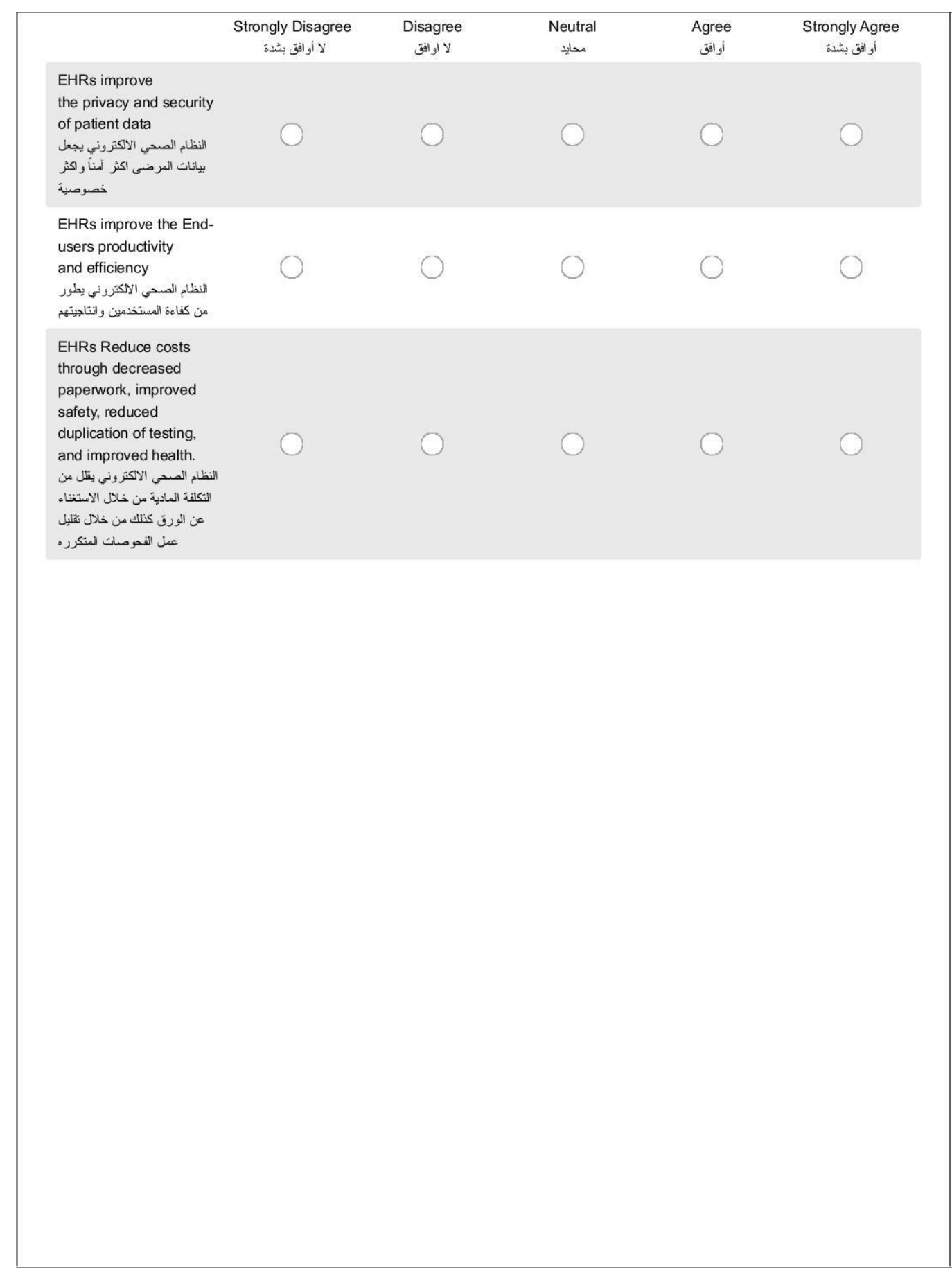




\begin{tabular}{|c|c|c|c|c|c|}
\hline & $\begin{array}{l}\text { Strongly Disagree } \\
\text { لا أوافق بشدة: }\end{array}$ & $\begin{array}{c}\text { Disagree } \\
\text { لاوافافق }\end{array}$ & $\begin{array}{c}\text { Neutral } \\
\text { محايد }\end{array}$ & $\begin{array}{l}\text { Agree } \\
\text { أوا قa }\end{array}$ & $\begin{array}{c}\text { Strongly Agree } \\
\text { كُوافق بشدة }\end{array}$ \\
\hline 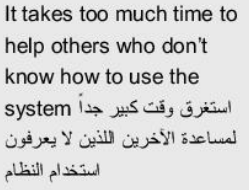 & & 0 & & & \\
\hline 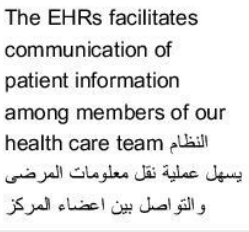 & & 0 & 0 & & 0 \\
\hline 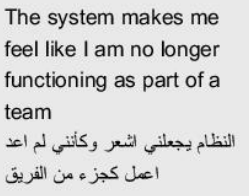 & & & $C$ & & \\
\hline 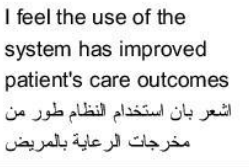 & & & & & \\
\hline 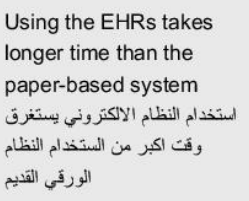 & & & & & \\
\hline 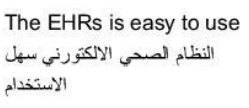 & 0 & 0 & 0 & 0 & 0 \\
\hline 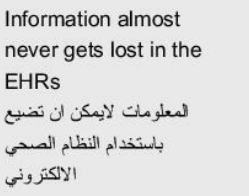 & & & & & \\
\hline 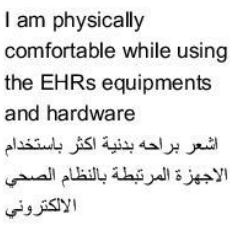 & $D$ & 0 & 0 & 0 & 0 \\
\hline
\end{tabular}




\begin{tabular}{|c|c|c|c|c|c|}
\hline & $\begin{array}{l}\text { Strongly Disagree } \\
\text { لا أوافق بشدة: }\end{array}$ & $\begin{array}{c}\text { Disagree } \\
\text { لاوافافق }\end{array}$ & $\begin{array}{c}\text { Neutral } \\
\text { محايد }\end{array}$ & $\begin{array}{l}\text { Agree } \\
\text { أوا قa }\end{array}$ & $\begin{array}{c}\text { Strongly Agree } \\
\text { كُوافق بشدة }\end{array}$ \\
\hline 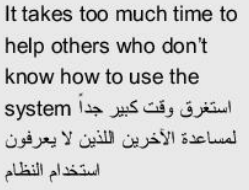 & & 0 & & & \\
\hline 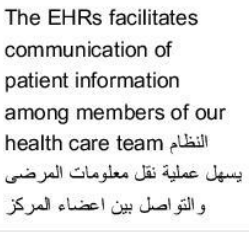 & & 0 & 0 & & 0 \\
\hline 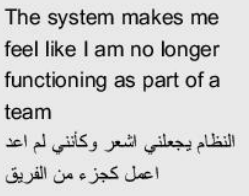 & & & $C$ & & \\
\hline 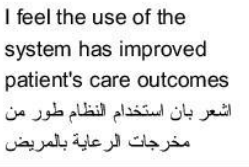 & & & & & \\
\hline 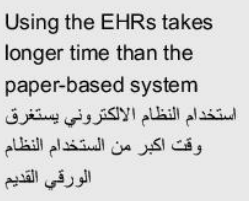 & & & & & \\
\hline 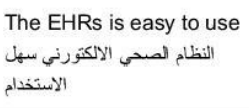 & 0 & 0 & 0 & 0 & 0 \\
\hline 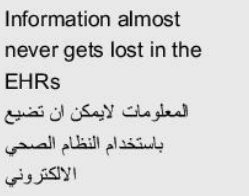 & & & & & \\
\hline 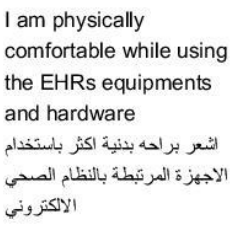 & $D$ & 0 & 0 & 0 & 0 \\
\hline
\end{tabular}




\begin{tabular}{|c|c|c|c|c|c|}
\hline & $\begin{array}{l}\text { Strongly Disagree } \\
\text { لا أوافق بشدة }\end{array}$ & $\begin{array}{c}\text { Disagree } \\
\text { لا لاوافق }\end{array}$ & $\begin{array}{c}\text { Neutral } \\
\text { محايد }\end{array}$ & $\begin{array}{l}\text { Agree } \\
\text { أوا قa }\end{array}$ & $\begin{array}{c}\text { Strongly Agree } \\
\text { كُوافق بشدة }\end{array}$ \\
\hline 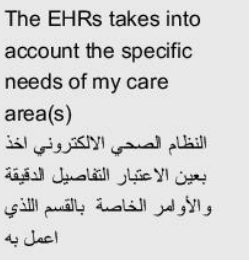 & & & & & \\
\hline 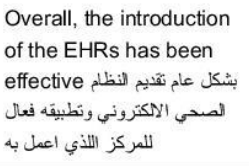 & & 0 & 0 & & ) \\
\hline 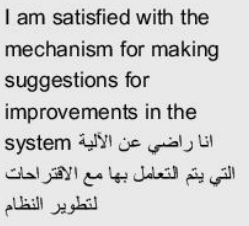 & & 0 & 0 & $\mathrm{O}$ & D \\
\hline 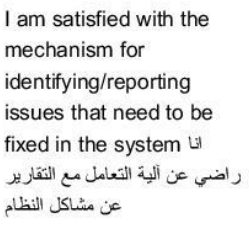 & & ) & 0 & & ) \\
\hline 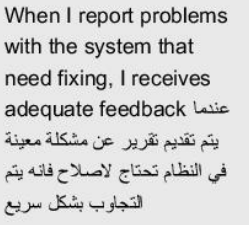 & & 0 & 0 & & ) \\
\hline
\end{tabular}




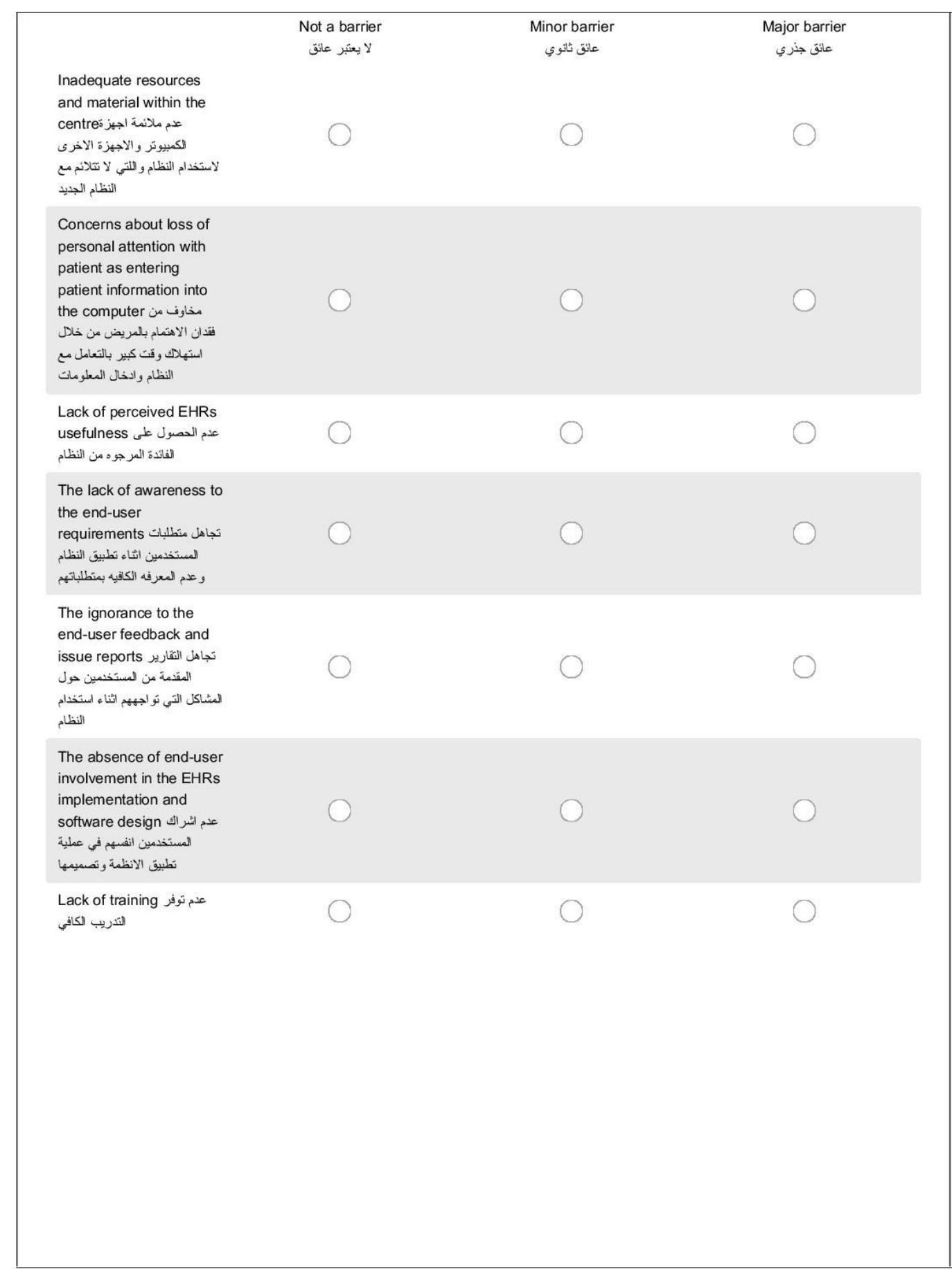


7. Open-end questions

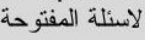

the purpose of this section is to obtain rich information and suggestions to help project team make better decision to implement the EHRs

الغرض من هذا القسم هو الحصول على معلومات اكثر شمولية بالاضافة إلى نصانحة لمساعدة متخذين القرار لانجاح تطبيق الاظظمة الاككترونية

12. What are the main things you like most about EHRs?

ماهي اهم الامور التي اعجبتك في النظام الصحي الاكتروني؟

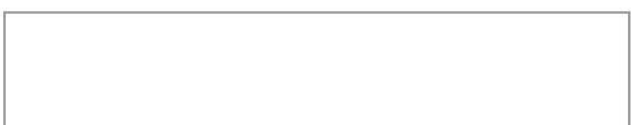

13. Do you have any recommendations to the decision makers to improve the implementation of the EHRs?

هل لديك اية نصانح لنتخنين القر ار بالوز ارة لتطوير تطبيق واستخدام النظام الصحي الالكتروني؟ 


\subsection{Data collection instrument for Study Four}

You have been chosen to be interviewed because you are currently involved in the Electronic Health Record system implementation project. The interview is part of a research study focused on implementing the EHRs in the Saudi PHCs. During the interview I will ask you about your professional background, how much you come into contact with the EHR system implementation process, how you plan to implement the EHRs, and what the procedures that you take into account to insure the successful implementation. The duration of this interview is estimated to take between 20 to 40 minutes.

Voluntary participation: It is voluntary to participate in the study and you can refrain from participation at any time without having to explain why. There is no reimbursement for participation.

Confidentiality: The researcher will handle the data anonymously and with confidentiality. Access to primary reported material is restricted to the researcher only (your employer will not have access to your answers). Readers of reports from the study will not be able to identify individual responses.

Data security: The researcher will do the best effort to ensure the security of your data by extracting all the needed information form the audio recorder and transcript the data into Microsoft Word and then will use appropriate software to analyse them (e.g. SPSS $\& \mathrm{~N}$-vivo). The researcher will lock the laptop with strong password and strong internet protection software will be installed. 


\section{$\underline{\text { Interview Consent Form }}$}

This consent form outlines my rights as a participant in the study of "Implementing the Electronic Health Record system on the organisation level" conducted by Haitham Ali Alzghaibi, College of Medicine, Swansea University, United Kingdom.

The interview will explore my experience and knowledge about

I understand that

Taking part in this study is entirely voluntary.

It is my right to decline to answer any question that I am asked.

I am free to end the interview at any time.

I may request that the interview not be taped.

My name and identity will remain confidential in any publications or discussions.

My name will not appear on any tapes or transcripts resulting from the interview.

I HAVE READ THIS CONSENT FORM. I HAVE HAD A CHANCE TO ASK QUESTIONS CONCERNING ANY AREAS THAT I DID NOT UNDERSTAND.

(Signature of Interviewee)

(Printed name of Interviewee)

(Date)

You may decline to participate in this study. You may end your participation in this study at any time. Maintaining your anonymity is a priority and every practical precaution will be taken to disguise your identity. There will not be any identifying information on audiotapes or transcripts of this interview. I will not allow anyone other than the research advisor to hear any audiotape of your voice or review a transcript of this interview. All materials generated from your interview (e.g., audiotapes and transcripts) will remain in my direct physical possession.

(Signature of Interviewer and Date)

- Interviewee code: 
- Position:

\section{Interview guide}

- What are the main Elements included in the plan?

- Can you please describe to me your plan to implement the EHRs in the PHCs including implementation timeline?

- Can you please describe how you decided to implement the EHRS in the PHCs?

- Can you please describe the level of the PHCs readiness to implement the EHRS?

- Was there any readiness assessment?

- Do you think PHCs ready to the EHRS implementation? Why?

- Did you consider the organisation structure and workflow redesign to meet the new changes?

- Can you please describe how you decided to select the project team and what are the criteria you took into account?

- Was the champions involve in the project team?

- What steps you take into consideration to ensure adequate communication between project team in all phases?

- Can you please describe the role of the leadership and management in the implementation planning and process?

- Was there any involvement from seniors and top managements in the EHRS implementation? Can you please describe their role in the implementation?

- Do you think the CM influence the EHRS implementation? how this type of management influences the implementation of the EHRS in the PHCs?

- In your opinion, what are the main factors influenced directly by the CM?

- In which way the CM influence these factors?

- Was there training for end-users?

- Can you please describe how you decided to provide training to the end-users of the EHRS?

- Can you please describe how you decided to provide technical support to the end-users of the EHRS?

- Have you been provided sufficient budget for this project?

- Can you please describe how FR influence the EHRS implementation in the Saudi PHCs?

- In your opinion, what are the main factors influenced directly by the FR?

- In which way the FR influence these factors? 
- Can you please describe how data security and patient's confidentiality were considers?

- what steps you take into account to ensure the systems are secure and patient data are confidential?

- Can you please describe how you decided to select the EHRS?

- what are the main criterions you considered while system selection?

- Has the system selected yet?

- In the idea, what would help to ensure the system is easy to use? and what steps you take into account to improve the system usability

- what would help to ensure efficiency of the EHRS? and what steps you take into account to improve the system efficiency

- What steps have you taken into account to ensure the EHRS will be interoperable with other systems in the Saudi $\mathrm{MOH}$ ?

- Can you please describe how you decided to involve end-users?

- Were adequate time and resources provided to support end-users involvement?

- Were the aim of the project and other implementation procedures clear to the end-users?

- Have you got any feedback from the end-user on the implemented systems?

- Can you please describe the level of end-user's satisfaction?

- Was there any resistance?

- Were there any staffing issues?

- Can you please tell me about the barriers and challenges during the implementation of the EHRs in the PHCs in SA?

- Were there any difficulties at technical level

- What steps you take into account to overcome these barriers?

- Can you please tell me about the facilitators to implement the EHRs in PHCs in SA?

- Is there any think else that you would like to add to improve the EHRS implementation?

- Do you have any recommendations to enhance the success of the EHRS for this project and other projects in the future?

- Is there anything you want to tell me at this time? 


\section{Appendices B}

Table 11.8.1: Kruskal-Wallis Test for occupation

\begin{tabular}{|l|l|r|r|}
\hline \multirow{3}{*}{$\begin{array}{l}\text { Positive } \\
\text { attitude }\end{array}$} & Physician & 24 & 67.27 \\
\cline { 2 - 4 } & Manager & 35 & 105.33 \\
\cline { 2 - 4 } & Nurse & 62 & 98.78 \\
\cline { 2 - 4 } & Lap technician & 12 & 131.04 \\
\cline { 2 - 4 } & Pharmacist & 20 & 124.63 \\
\cline { 2 - 4 } & Radiologist & 9 & 75.22 \\
\hline \multirow{2}{*}{} & Dentist & 13 & 94.50 \\
\hline \multirow{2}{*}{} & Receptionist & 19 & 79.95 \\
\hline & Total & 194 & \\
\hline
\end{tabular}


Table 11.8.2: Number of articles included in the literature

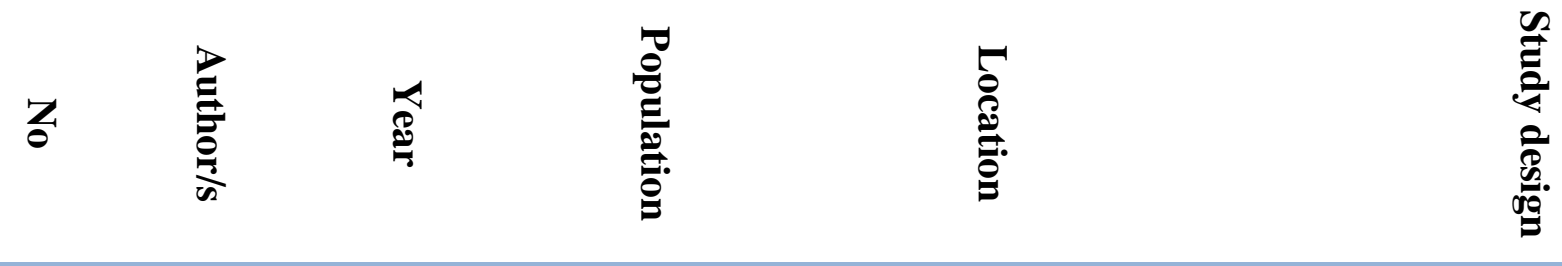

\begin{tabular}{|c|c|c|c|c|c|}
\hline 1 & Adler K. G. & 2004 & $\begin{array}{l}\text { Primary and } \\
\text { secondary care }\end{array}$ & USA & N/A \\
\hline 2 & Adler K. G. & 2007 & $\begin{array}{l}\text { Primary and } \\
\text { secondary care }\end{array}$ & USA & N/A \\
\hline 3 & $\begin{array}{l}\text { Ahmadian } \\
\text { et al., }\end{array}$ & 2014 & Secondary care & Iran & Quantitative/survey \\
\hline 4 & $\begin{array}{l}\text { Ajami and } \\
\text { Arab- } \\
\text { Chadegani }\end{array}$ & 2013 & $\begin{array}{l}\text { Primary and } \\
\text { secondary care }\end{array}$ & Iran & Review of literature \\
\hline 5 & Ajami et al., & 2011 & $\begin{array}{l}\text { Primary and } \\
\text { secondary care }\end{array}$ & Iran & Review of literature \\
\hline 6 & $\begin{array}{l}\text { Alasmary et } \\
\text { al., }\end{array}$ & 2014 & secondary care & Saudi Arabia & Quantitative/survey \\
\hline 7 & Aldosari B. & 2014 & secondary care & Saudi Arabia & Quantitative/survey \\
\hline 8 & $\begin{array}{l}\text { Alkraiji et } \\
\text { al., }\end{array}$ & 2013 & secondary care & Saudi Arabia & Qualitative/ interviews \\
\hline 9 & $\begin{array}{l}\text { Ancker et } \\
\text { al., }\end{array}$ & 2013 & Primary care & USA & Mixed-methods/ survey and interviews \\
\hline 10 & $\begin{array}{l}\text { Arvanitis, T. } \\
\text { N. }\end{array}$ & 2014 & $\begin{array}{l}\text { Primary and } \\
\text { secondary care }\end{array}$ & UK & Keynote \\
\hline 11 & Ash et a., & 2012 & Secondary Care & USA & Qualitative/ interviews and Observatio \\
\hline
\end{tabular}




\begin{tabular}{|c|c|c|c|c|c|}
\hline 12 & $\begin{array}{l}\text { Ash and } \\
\text { Bates }\end{array}$ & 2005 & Secondary Care & USA & Report \\
\hline 13 & $\begin{array}{l}\text { Bani-Issa et } \\
\text { al., }\end{array}$ & 2016 & Secondary Care & $\begin{array}{l}\text { United Arab } \\
\text { Emirates }\end{array}$ & Quantitative \\
\hline 14 & Bates et al., & 2003 & Primary care & USA & Review of literature \\
\hline 15 & Biruk et al., & 2014 & Secondary Care & Ethiopia & Qualitative \\
\hline 16 & $\begin{array}{l}\text { Boonstra } \\
\text { and } \\
\text { Broekhuis }\end{array}$ & 2010 & $\begin{array}{l}\text { Primary and } \\
\text { secondary care }\end{array}$ & Netherlands & Systematic review \\
\hline 17 & $\begin{array}{l}\text { Boonstra et } \\
\text { al., }\end{array}$ & 2014 & Secondary Care & Netherlands & Systematic review \\
\hline 18 & $\begin{array}{l}\text { Carayon et } \\
\text { al., }\end{array}$ & 2009 & Primary care & USA & $\begin{array}{l}\text { Mixed-methods/survey, interviews and } \\
\text { documentation analysis }\end{array}$ \\
\hline 19 & $\begin{array}{l}\text { Cresswell } \\
\text { and } \\
\text { Sheikh }\end{array}$ & 2013 & $\begin{array}{l}\text { Primary and } \\
\text { secondary care }\end{array}$ & UK & Review of systematic reviews \\
\hline 20 & $\begin{array}{l}\text { Cresswell et } \\
\text { al., }\end{array}$ & 2013 & Secondary care & UK & Review of literature \\
\hline 21 & $\begin{array}{l}\text { Cucciniello } \\
\text { et al., }\end{array}$ & 2015 & Secondary care & UK & Qualitative/ documentation analysis \\
\hline 22 & $\begin{array}{l}\text { DesRoches } \\
\text { et al., }\end{array}$ & 2012 & Secondary care & USA & Quantitative/ survey \\
\hline 23 & $\begin{array}{l}\text { Fernandez- } \\
\text { Aleman et } \\
\text { al., }\end{array}$ & 2013 & $\begin{array}{l}\text { Primary and } \\
\text { secondary care }\end{array}$ & Spain & Systematic review \\
\hline 24 & Fritz et al., & 2015 & $\begin{array}{l}\text { Primary and } \\
\text { secondary care }\end{array}$ & Germany & Systematic review \\
\hline 25 & $\begin{array}{l}\text { Fullerton et } \\
\text { al., }\end{array}$ & 2006 & $\begin{array}{l}\text { Primary and } \\
\text { secondary care }\end{array}$ & USA & Review of literature \\
\hline
\end{tabular}




\begin{tabular}{|c|c|c|c|c|c|}
\hline 26 & $\begin{array}{l}\text { Gagnon et } \\
\text { al., }\end{array}$ & $2014 \mathrm{a}$ & $\begin{array}{l}\text { Primary and } \\
\text { secondary care }\end{array}$ & Canada & Systematic review \\
\hline 27 & $\begin{array}{l}\text { Gagnon et } \\
\text { al., }\end{array}$ & 2016 & $\begin{array}{l}\text { Primary and } \\
\text { secondary care }\end{array}$ & Canada & Qualitative/ semi-structure interviews \\
\hline 28 & $\begin{array}{l}\text { Gagnon et } \\
\text { al., }\end{array}$ & 2012 & $\begin{array}{l}\text { Primary and } \\
\text { secondary care }\end{array}$ & Canada & Systematic review \\
\hline 29 & $\begin{array}{l}\text { Gagnon et } \\
\text { al., }\end{array}$ & $2014 b$ & $\begin{array}{l}\text { Primary and } \\
\text { secondary care }\end{array}$ & Canada & Systematic review \\
\hline 30 & $\begin{array}{l}\text { Ghazisaeidi } \\
\text { et al., }\end{array}$ & 2014 & $\begin{array}{l}\text { Primary and } \\
\text { secondary care }\end{array}$ & Iran & Review of literature \\
\hline 31 & $\begin{array}{l}\text { Gordon et } \\
\text { al., }\end{array}$ & 2015 & Primary care & USA & Quantitative/ survey \\
\hline 32 & $\begin{array}{l}\text { Hartzler et } \\
\text { al., }\end{array}$ & 2013 & $\begin{array}{l}\text { Primary and } \\
\text { secondary care }\end{array}$ & USA & Review of literature \\
\hline 33 & $\begin{array}{l}\text { Hoerbst and } \\
\text { Schweitzer }\end{array}$ & 2015 & $\begin{array}{l}\text { Primary and } \\
\text { secondary care }\end{array}$ & Austria & Systematic review \\
\hline 34 & $\begin{array}{l}\text { Holanda et } \\
\text { al., }\end{array}$ & 2012 & Primary care & Brazil & Quantitative/ questionnaire \\
\hline 35 & $\begin{array}{l}\text { Ingebrigtsen } \\
\text { et al., }\end{array}$ & 2014 & $\begin{array}{l}\text { Primary and } \\
\text { secondary care }\end{array}$ & Australia & Systematic review \\
\hline 36 & Jardim & 2013 & $\begin{array}{l}\text { Primary and } \\
\text { secondary care }\end{array}$ & Portugal & Review of literature \\
\hline 37 & $\begin{array}{l}\text { Kemper et } \\
\text { al., }\end{array}$ & 2006 & Primary care & USA & Quantitative/ survey \\
\hline 38 & $\begin{array}{l}\text { Keshavjee } \\
\text { et al., }\end{array}$ & 2006 & $\begin{array}{l}\text { Primary and } \\
\text { secondary care }\end{array}$ & Canada & Systematic review \\
\hline 39 & $\begin{array}{l}\text { Khajouei et } \\
\text { al., }\end{array}$ & 2011 & Secondary care & Netherlands & Quantitative/ questionnaire \\
\hline 40 & Kruse et al., & 2014 & $\begin{array}{l}\text { Primary and } \\
\text { secondary care }\end{array}$ & USA & Systematic review \\
\hline
\end{tabular}




\begin{tabular}{|c|c|c|c|c|c|}
\hline 41 & Kruse et al., & $2016 a$ & $\begin{array}{l}\text { Primary and } \\
\text { secondary care }\end{array}$ & USA & Systematic review \\
\hline 42 & Kruse et al., & $2016 b$ & $\begin{array}{l}\text { Primary and } \\
\text { secondary care }\end{array}$ & USA & Systematic review \\
\hline 43 & Kruse et al., & 2015 & $\begin{array}{l}\text { Primary and } \\
\text { secondary care }\end{array}$ & USA & Systematic review \\
\hline 44 & $\begin{array}{l}\text { Lennon et } \\
\text { al., }\end{array}$ & 2017 & $\begin{array}{l}\text { Primary and } \\
\text { secondary care }\end{array}$ & UK & Mixed-methods \\
\hline 45 & $\begin{array}{l}\text { Lorenzi et } \\
\text { al., }\end{array}$ & 2009 & Primary care & USA & Review of literature \\
\hline 46 & $\begin{array}{l}\text { Ludwick } \\
\text { and } \\
\text { Doucette }\end{array}$ & $2009 a$ & Primary care & Canada & Systematic review \\
\hline 47 & $\begin{array}{l}\text { Ludwick } \\
\text { and } \\
\text { Doucette }\end{array}$ & $2009 b$ & Primary care & Canada & Qualitative/ semi-structure interviews \\
\hline 48 & Luna et al., & 2014 & $\begin{array}{l}\text { Primary and } \\
\text { secondary care }\end{array}$ & Argentina & Review of literature \\
\hline 49 & $\begin{array}{l}\text { McAlearney } \\
\text { et al., }\end{array}$ & 2013 & Secondary care & USA & Qualitative/ focus groups and interviev \\
\hline 50 & $\begin{array}{l}\text { Middleton } \\
\text { et al., }\end{array}$ & 2013 & $\begin{array}{l}\text { Primary and } \\
\text { secondary care }\end{array}$ & USA & Review of literature \\
\hline 51 & $\begin{array}{l}\text { Ovretveit et } \\
\text { al., }\end{array}$ & 2007 & secondary care & Swedish & $\begin{array}{l}\text { Qualitative/ semi-structured interviews } \\
\text { documentation }\end{array}$ \\
\hline 52 & Pare et al., & 2008 & $\begin{array}{l}\text { Primary and } \\
\text { secondary care }\end{array}$ & Canada & Review of literature and Delphi survey \\
\hline 53 & Poon et al., & 2004 & Secondary care & USA & Qualitative/ semi-structure interviews \\
\hline 54 & Rao et al., & 2011 & Primary care & USA & Quantitative/ survey \\
\hline
\end{tabular}




\begin{tabular}{|c|c|c|c|c|c|}
\hline 55 & $\begin{array}{l}\text { Rezaeibagha } \\
\text { et al., }\end{array}$ & 2015 & $\begin{array}{l}\text { Primary and } \\
\text { secondary care }\end{array}$ & Australia & Systematic review \\
\hline 56 & Rizer et al., & 2015 & Secondary care & USA & Case study \\
\hline 57 & Ross et al., & 2016 & $\begin{array}{l}\text { Primary and } \\
\text { secondary care }\end{array}$ & UK & Systematic review \\
\hline 58 & Ross et al., & 2010 & Primary care & USA & Mixed-methods \\
\hline 59 & $\begin{array}{l}\text { Safdari et } \\
\text { al., }\end{array}$ & 2015 & Secondary care & Iran & Quantitative/ questionnaire \\
\hline 60 & $\begin{array}{l}\text { Sanchez et } \\
\text { al., }\end{array}$ & 2005 & $\begin{array}{l}\text { Primary and } \\
\text { secondary care }\end{array}$ & France & Qualitative/ semi-structure interviews \\
\hline 61 & Scott et al., & 2005 & Primary care & USA & Qualitative/ semi-structure interviews \\
\hline 62 & Simon et al., & 2007 & $\begin{array}{l}\text { Primary and } \\
\text { secondary care }\end{array}$ & USA & Quantitative/ survey \\
\hline 63 & Simon et al., & 2013 & Secondary care & USA & Qualitative/observations and interview \\
\hline 64 & Singh et al., & 2012 & Primary care & USA & Quantitative/ survey \\
\hline 65 & Slight et al., & 2014 & Secondary care & UK & Qualitative/ semi-structure interviews \\
\hline 66 & Terry et al., & 2009 & Primary care & Canada & Qualitative/ semi-structure interviews \\
\hline 67 & Terry et al., & 2008 & Primary care & Canada & $\begin{array}{l}\text { Three qualitative studies/ semi-structu } \\
\text { interviews }\end{array}$ \\
\hline 68 & Walji et al., & 2009 & Secondary care & USA & $\begin{array}{l}\text { Mixed methods/ documentation analys } \\
\text { interviews and survey }\end{array}$ \\
\hline 69 & Walker & 2006 & $\begin{array}{l}\text { Primary and } \\
\text { secondary care }\end{array}$ & UK & N/A \\
\hline 70 & $\begin{array}{l}\text { Wang and } \\
\text { Biedermann }\end{array}$ & 2012 & $\begin{array}{l}\text { Primary and } \\
\text { secondary care }\end{array}$ & USA & Quantitative/survey \\
\hline 71 & $\begin{array}{l}\text { Whitacre } \\
\text { and } \\
\text { Williams }\end{array}$ & 2015 & $\begin{array}{l}\text { Primary and } \\
\text { secondary care }\end{array}$ & USA & Quantitative/survey \\
\hline
\end{tabular}




\begin{tabular}{|c|c|c|c|c|c|}
\hline 72 & $\begin{array}{l}\text { Yoon- } \\
\text { Flannery et } \\
\text { a., }\end{array}$ & 2008 & Secondary care & USA & Qualitative/ semi-structure interviews \\
\hline 73 & Yusif et al., & 2017 & $\begin{array}{l}\text { Primary and } \\
\text { secondary care }\end{array}$ & Australia & Systematic review \\
\hline 74 & $\begin{array}{l}\text { Yusof and } \\
\text { Aziz }\end{array}$ & 2015 & Secondary care & Malaysia & $\begin{array}{l}\text { Qualitative/ interviews, observations a } \\
\text { document analysis }\end{array}$ \\
\hline 75 & Yusof et al., & 2008 & $\begin{array}{l}\text { Primary and } \\
\text { secondary care }\end{array}$ & Malaysia & Review of literature \\
\hline 76 & $\begin{array}{l}\text { Zahabi et } \\
\text { al., }\end{array}$ & 2015 & $\begin{array}{l}\text { Primary and } \\
\text { secondary care }\end{array}$ & USA & Review of literature \\
\hline
\end{tabular}

